\title{
17. GEOCHEMISTRY OF LATE CENOZOIC SEDIMENTS OF THE BLACK SEA, LEG 42B
}

\author{
E. M. Emelyanov, A. P. Lisitzin, K. M. Shimkus, E. S. Trimonis, V. K. Lukashev, V. N. Lukashin, A. Yu. Mitropolskiy, \\ and M. F. Pilipchuk
}

P. P. Shirshov Institute of Oceanology, Academy of Science, USSR, Kaliningrad, U.S.S.R.

\section{INTRODUCTION}

Geochemical investigations were carried out at the three sites $(379,380$, and 381$)$ and covered the entire stratigraphic section. The main macrocomponents $\left(\mathrm{CaCO}_{3}, \mathrm{C}_{\text {org }}, \mathrm{SiO}_{2}\right.$ [amorph], $\left.\mathrm{Fe}, \mathrm{Mn}, \mathrm{Ti}, \mathrm{P}, \mathrm{K}, \mathrm{Na}\right)$ as well as of 24 minor elements $(\mathrm{Cu}, \mathrm{Zn}, \mathrm{Ni}, \mathrm{Co}, \mathrm{Cr}, \mathrm{V}$, $\mathrm{Cd}, \mathrm{Li}, \mathrm{Rb}, \mathrm{Cs}, \mathrm{Sn}, \mathrm{Se}, \mathrm{Mo}, \mathrm{W}, \mathrm{F}, \mathrm{B}, \mathrm{La}, \mathrm{Th}, \mathrm{Hf}, \mathrm{Sc}$, $\mathrm{Eu}, \mathrm{Sb}, \mathrm{Ce}, \mathrm{Ta}$ ) were determined. Different sediment types were studied within each stratigraphic unit.

Cores were taken near the drill sites for comparison of the older sediments with those of Recent and late Quaternary age. Study methods were similar.

Analyses were performed in the following institutions: $\mathrm{CaCo}_{3}, \mathrm{Corg}, \mathrm{Fe}, \mathrm{Mn}, \mathrm{Ti}, \mathrm{K}, \mathrm{Na}, \mathrm{Ni}, \mathrm{Cr}$, $\mathrm{Cu}, \mathrm{Zn}$, from Site 379 and $\mathrm{A}, \mathrm{K}, \mathrm{Na}, \mathrm{Cu}, \mathrm{Zn}, \mathrm{Ni}, \mathrm{Cr}$ from Sites 380 and 381 in the Laboratory of Atlantic Geology, Oceanology Institute, USSR Academy of Sciences, Atlantic Department, Kaliningrad (E. M. Emelyanov); $\mathrm{CaCo}_{3}, \mathrm{Corg}, \mathrm{Fe}, \mathrm{Mn}, \mathrm{Ti}, \mathrm{P}, \mathrm{Mo}, \mathrm{W}, \mathrm{Se}$ from Sites 380 and 381 in the Laboratory of South Seas Geology, Oceanology Institute, USSR Academy of Sciences, Southern Department, Gelendjik (K. M. Shimkus); $\mathrm{Li}, \mathrm{Rb}, \mathrm{Cs}, \mathrm{Pb}, \mathrm{Ag}$, and some of the $\mathrm{Sn}$ analyses in the Institute of Geological Sciences, USSR Academy of Sciences, Kiev (A. Yu. Mitropolskiy); B, F in total sediment, $\mathrm{K}, \mathrm{B}, \mathrm{Ni}, \mathrm{Co}, \mathrm{Cr}, \mathrm{V}$ in the $0.001 \mathrm{~mm}$ fraction, and $\mathrm{Ni}, \mathrm{Co}, \mathrm{Cr}, \mathrm{V}$ in total sediment in the Geochemistry and Geophysics Institute, USSR Academy of Sciences, Minsk (V. K. Lukashev); Tr, Hf, Sc, etc. in the P. P. Shirshov Oceanological Institute, USSR Academy of Sciences, Moscow (A. P. Lisitzin).

We acknowledge help from technicians of the Atlantic Geology Laboratory: G. V. Cheremissina, N. G. Grudinova, and N. D. Shibkova. Mathematical treatment of the data was carried out by $\mathrm{O}$. V. Leontyeva and V. Vasilenko.

Sections I and III were written by all authors; Sections II and V were written by E. M. Emelyanov, A. P. Lisitzin, K. M. Shimkus, and E. S. Trimonis.

The data make it possible to describe the main distributions of macrocomponents and microelements in the section and their relations to the processes of sedimentation and diagenesis.

\section{METHODS}

$\mathrm{CaCO}_{3}$ and $\mathrm{C}_{\text {org }}$ were analyzed by an improved volumetric method (Sokolov and Sokolova, 1975); a faster method was occasionally used for $\mathrm{CaCO}_{3}$. Amorphous silica $\left(\mathrm{SiO}_{2}\right.$ amorph) was studied on individual samples by sodium, and $\mathrm{P}$ was determined colorimetrically (Ponomariov, 1961, p. 140-141). Fe, $\mathrm{Mn}, \mathrm{Cu}, \mathrm{Zn}, \mathrm{Ni}, \mathrm{Cr}, \mathrm{Cd}, \mathrm{K}, \mathrm{Na}$, and $\mathrm{Ti}$ were determined on single samples after acidic decomposition (Poluektov, 1959, p. 140-141). Concentration of $\mathrm{K}$ and $\mathrm{Na}$ in solution was measured using the flame M-3 photometer, $\mathrm{Ti}$ was measured using the FEK-56 photoelectrocolimeter, and concentration of all other elements was measured using an atomic absorption spectrophotometer. Control was based on the geological standards of the USSR (SG-1, SYD-1, ST-1) and the GDR (Granite, Basalt, Tonschieferm Kalkstein), as well as on intralaboratory standards.

The elements $\mathrm{Ba}, \mathrm{Zr}, \mathrm{V}, \mathrm{Be}, \mathrm{Ge}, \mathrm{Mo}, \mathrm{Sn}, \mathrm{Ni}$, and $\mathrm{Cr}$ were determined (for control) by quantitative spectrographic method (Meishtas, 1969).

Quantitative determination of $\mathrm{B}, \mathrm{Ni}, \mathrm{Co}, \mathrm{Cr}, \mathrm{V}$ in total sediment samples and in the $<0.001 \mathrm{~mm}$ fraction was made by the emission spectral method using the PCS-2 diffraction grating spectrograph (Carl Zeiss Yena) and photographic recording of spectra. Samples and standards were buffered in the ratio $1: 2$ by spectrally pure coal powder containing $0.2 \%$ of palladium chloride. Palladium was introduced as an internal standard. Analytical lines of elements are as follows: B - $249 \mathrm{n}$; Ni - $305.5 \mathrm{n}$; Co $-354.4 \mathrm{n}$; Cr $301.4 \mathrm{n} ; \mathrm{V}-292.4 \mathrm{n}$; Pd $-247.6 \mathrm{n}$. The geological standard of JRGJREDMET was used for control of analysis results. $\mathrm{Li}, \mathrm{Rb}, \mathrm{Cs}, \mathrm{Pb}, \mathrm{Sn}$, and $\mathrm{Ag}$ were analyzed by the quantitative emission spectral method. The spectrum of a sample was excited by introduction of powder into the plasma discharge, using the USA-4 instrument (spilling).

Artificial mixtures were used for preparation of standards. Standards approximated the bulk composition of the analyzed samples. The main standard was prepared taking into account the highest elements in investigated samples. Standards were made by dilution of the main standard by the Tongeren method $(1.0 \%, 0.315 \%, 0.0315 \%, 0.01 \%, 0.00315 \%$, $0.001 \%, 0.000315 \%$, and $0.0001 \%$ ). Background was used as an internal standard.

Analyzed samples and standards were photographed on a single plate two and three times, respectively. Photometering of standards and samples was performed on the MF-2 microphotometer using the analytical line of $\mathrm{Li}$ at $8126.52 \AA$. Analytical lines for photometering were chosen with allowance for concentration sensitivity and a content of the element in a sample that produced blackening on a rectilinear part of the graduated graph. Content of the elements 
$\mathrm{Li}, \mathrm{Rb}, \mathrm{Cs}, \mathrm{Pb}, \mathrm{Sn}, \mathrm{Ag}$ was evaluated on graphs constructed on $\triangle \mathrm{S} / \mathrm{IgC}$ coordinates. Relative error is $10-15 \%$.

Fluorine content was determined by a rapid method for the determination of fluorine in small quantities in rocks. The sensitivity of the methods is $0.08 \mathrm{mg} / \mathrm{l}$. The procedure is: $0.3-0.5 \mathrm{~g}$ of sample is melted with onetenth of soda and $0.2 \mathrm{~g}$ of zinc oxide, followed by leaching with hot water. Fluorine was determined colorimetrically by zircon-alizarine varnish discoloration.

Boron was determined by spectrophotometry. Atomic-absorption and flame-photometry analyses were performed by Yu. O. Shaidurov, E. A. Ivanchenko; chemical analyses by Ts. H. Yablunovskaja, T. I. Khomina, L. A. Akulenko, E. G. Sokolva, V. S. Sokolov, O. I. Bondarenko, E. I. Fomichiova, N. V. Osadchaya, and E. S. Sevastyanova.

Neutron activation analysis (INAA) was used to determine $\mathrm{La}, \mathrm{Ce}, \mathrm{Eu}, \mathrm{Hf}, \mathrm{Ta}$, Th, and $\mathrm{Sb}$, and for control, Ni, Cr, and Fi (Figure 1). The analyses were done by N. V. Katargin.

The INAA complex consists of the following instruments: (1) reactor VVR-C; (2) semiconducting $\mathrm{Ge}(\mathrm{Li})$ detector $50 \mathrm{~cm}^{3}$ in volume with a carrying capacity of $2 \mathrm{kev}$ by $\mathrm{Co}^{60}$ ( $5 \mathrm{kev}$ at the instrument spectrum); (3) spectrometric amplifier (intensifier) "Langur"; (4) 512-channel analyzer LP-4050; and (5) the computer "Nairi-K."

Methods of analysis and identification of isotopes were based on A. D. Kurinov's methods used for analysis of bottom sediments in the Pacific and Indian oceans (Kurinov et al., 1974).

Samples (5-10 $\mathrm{mg}$ in weight packed in aluminum foil) were irradiated for 80 hours in a flow $\sim 10^{13} \mathrm{n} / \mathrm{cm}^{2}$ per sec. After 7-9 days treatment, the samples were repacked in new foil and the peak of La 141 was measured $(\mathrm{E} \alpha=1596 \mathrm{keV})$.

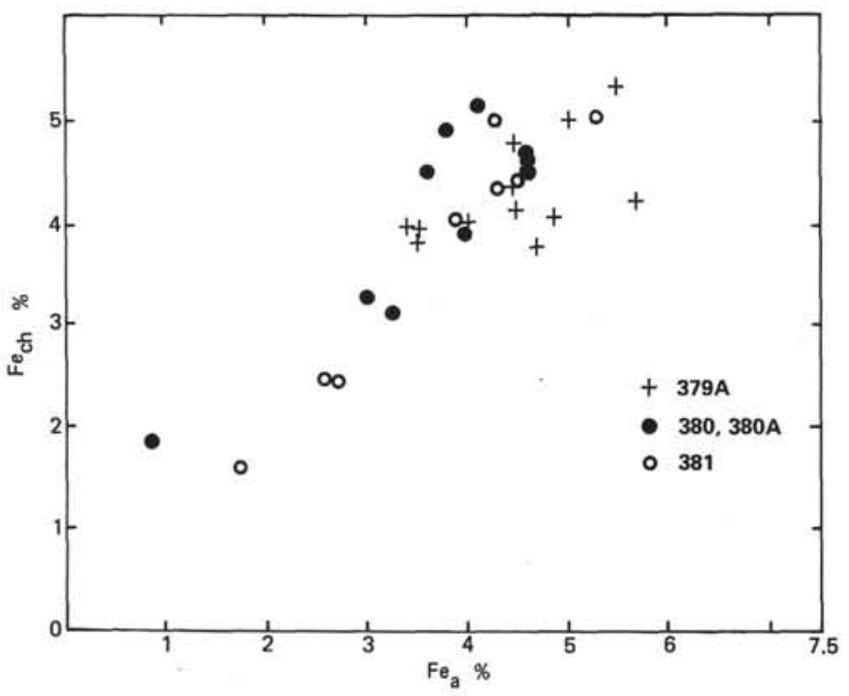

Figure 1. Iron contents in Hole $379 \mathrm{~A}$ sediments, studied by atomic absorption $\left(\mathrm{Fe}_{\mathrm{ch}}\right)$ and neutron activation $\left(\mathrm{Fe}_{a}\right)$; comparison of results is satisfactory.
Measurement of peaks of elements with long halflives was made 20-30 days after irradiation of samples. Radioisotope characteristics used for analytical purposes are shown in Table 1.

USGS rock standards (G SP-1, AGV-1, PCC-1) were used to measure standard intensities.

Precision of determination of those elements with sharp peaks ( $\mathrm{Sc}, \mathrm{Co}, \mathrm{Fe}, \mathrm{La}$ ) depends upon their nonuniformity in the standards, as well as the precision of the standards. Precision of $\mathrm{Eu}, \mathrm{Sb}, \mathrm{Ta}, \mathrm{Rb}$, and $\mathrm{Hf}$ is decreased because of signal characteristics. Statistical error for $\mathrm{Eu}$ is $10 \%$ to $20 \%$, for $\mathrm{Rb}$ and $\mathrm{Hf}, 20 \%$ to $40 \%$. $\mathrm{Ta}$ and $\mathrm{Sb}$ were not often determined at all, since their photopeaks are too weak and sink into the background. The observational limit for $\mathrm{Sb}$ is $0.5 \mathrm{ppm}$ and for $\mathrm{Ta}$, $0.2 \mathrm{ppm}$. The standard intensity for cesium was not determined by the standards, but calculated theoretically by its monitors, cobalt and antimony. Precision of determination of cesium is estimated to be about $30 \%$.

The non-uniformity of the elements' distribution in the sediments contributes about $5 \%$ to $10 \%$ to the reproducibility error (Kurinov, 1975).

Photopeaks of $\mathrm{Ge}, \mathrm{Rb}$, and $\mathrm{Cs}$ were not analyzed by computer; their intensity was determined by spectrogram. Determination precision and sensitivity of $\mathrm{Ce}$ depend on the interference of the $\mathrm{Fe}^{59}$ peak $(\mathrm{E}=$ $143 \mathrm{keV}$ ), as well as on the other peaks nearby. The determination precision of $\mathrm{Ce}$ is estimated to be $20 \%$ to $30 \%$; the observational limit is $20 \mathrm{ppm}$.

The photopeak of $\mathrm{Hf}$ in samples from Hole 379A and Site 380 is undistorted by the barium peak $131(E=496$ $\mathrm{keV}$ ), because less than $300 \mathrm{ppm}$ were recorded in these samples. There is more barium in Hole $380 \mathrm{~A}$ and Site 381 , therefore determination of $\mathrm{Hf}$ in some samples was not possible. In these cases, peak intensity was determined by spectrogram.

The determination of thorium and chromium was difficult because of the density of $\mathrm{Pa}^{233}$ and $\mathrm{Cr}^{51}$ peaks. It often happens that each isotope contributes almost equally to the doubled photopeak, so the spectrogram can be used to determine their concentrations; sometimes it is impossible to identify the elements individually. Precision is about $20 \%$ to $30 \%$.

Data on the size distribution and mineral composition of the sediments are taken from Trimonis et al. (this volume).

An infrared spectrometric method used by Levitan (personal communication) indicated that chemically determined $\mathrm{SiO}_{2}$ amorph contents are usually underestimated by a factor of $1.2-2.0$, especially at low concentrations. In our conversions a factor of 1.6 is used.

Mean chemical and mineral composition of Hole 379A sediments, as well as of paired element and mineral correlation were calculated, using the MINSK22 computer. In case of missing data for one of the components (elements) in paired correlations, the mean values by unit are taken.

For conversions to the so-called "clastic" product the following formula was applied: $100 \%-\left[\mathrm{CaCO}_{3}+\right.$ $(\mathrm{Corg} \times 2)+\left(\mathrm{SiO}_{2}\right.$ amorph $\left.\left.\times 1.6\right)\right]=t$, where $t$ is 
TABLE 1

Radioisotopes Used and Sensitivity and Precision of Analysis

\begin{tabular}{|c|c|c|c|c|c|c|}
\hline Element & $\begin{array}{l}\text { Radio- } \\
\text { isotope }\end{array}$ & $\underset{\text { (kev) }}{\mathrm{E}}$ & $\begin{array}{l}T 1 / 2 \\
\text { (days) }\end{array}$ & $\begin{array}{l}\text { Sensi- } \\
\text { tivity } \\
\text { (ppm) }\end{array}$ & $\begin{array}{l}\text { Precision } \\
(\%)\end{array}$ & Limit of Precision \\
\hline $\mathrm{Cr}$ & $\mathrm{Cr}^{51}$ & 320 & 27.7 & $20-60$ & $20-30$ & Interference of $\mathrm{Pa}^{233}$ \\
\hline Co & $\mathrm{Co}^{60}$ & 1173 & $5.26 \mathrm{yr}$ & 0.5 & 10 & Precision of standards \\
\hline Co & $\mathrm{Co}^{60}$ & 1332 & $5.26 \mathrm{yr}$ & 0.5 & 10 & \\
\hline $\mathrm{Eu}$ & $\mathrm{Eu}^{152}$ & 1408 & $12.1 \mathrm{yr}$ & 0.3 & $10-20$ & $\begin{array}{l}\text { Precision of standards, } \\
\text { statistical fluctuations }\end{array}$ \\
\hline $\mathrm{Fe}$ & $\mathrm{Fe}^{59}$ & 1291 & 44.5 & $0.1 \%$ & 10 & Precision of standards \\
\hline $\mathrm{Fe}$ & $\mathrm{Mn}^{54}$ & 835 & 314 & $0.3 \%$ & 15 & \\
\hline La & $\mathrm{La}^{140}$ & 1596 & 1.67 & 5 & 10 & Precision of standards \\
\hline $\mathrm{Sb}$ & $\mathrm{Sb}^{124}$ & 1691 & 60.4 & 0.5 & 30 & Statistical fluctuations \\
\hline $\mathrm{Sc}$ & $\mathrm{Sc}^{46}$ & 889 & 83.8 & 0.1 & 10 & Precision of standards \\
\hline Cs & $\mathrm{Cs}^{134}$ & 796 & $2.1 \mathrm{yr}$ & 0.5 & $20-30$ & $\begin{array}{l}\text { Standardization on } \\
\text { monitors }\end{array}$ \\
\hline $\mathbf{R b}$ & $\mathrm{Rb}^{86}$ & 1078 & 21 & 40 & $20-40$ & Statistical fluctuations \\
\hline $\mathrm{Ta}$ & $\mathrm{Ta}^{182}$ & 1221 & 115.1 & 0.2 & $20-40$ & \\
\hline Th & $\mathrm{Pa}^{233}$ & 311 & 27 & $3-5$ & $20-30$ & Interference of $\mathrm{Cr}^{51}$ \\
\hline $\mathrm{Hf}$ & $\mathrm{Hf}^{181}$ & 482 & 42.45 & $0.5-1$ & $20-40$ & $\begin{array}{l}\text { Statistical fluctuations } \\
\text { and interference of } \mathrm{Ba}^{13}\end{array}$ \\
\hline
\end{tabular}

terrigenous clastic-clay material. A factor of 1.8 is used in converting to organic matter. Since Corg contents in the studied sections is low, this factor is rounded to 2 for convenience. Corg and $\mathrm{SiO}_{2}$ amorph contents were converted to clastic product by the formulas: $100 \%$ $\left[\mathrm{CaCO}_{3} \times\left(\mathrm{SiO}_{2}\right.\right.$ amorph $\left.\left.\times 1.6\right)\right]=t$, and $100 \%-$ $\left[\mathrm{CaCO}_{3}+(\mathrm{C}\right.$ org $\times 2)=t$, respectively.

Mean components and element values by units are converted to clastic products based on mean component and element data from natural (dry) sediments of the unit given.

All microcomponent contents $\left(\mathrm{CaCO}_{3}, \mathrm{SiO}_{2}\right.$ amorph, Corg, $\mathrm{Fe}, \mathrm{Mn}, \mathrm{Ti}, \mathrm{P}, \mathrm{K}, \mathrm{Na}$ ) are given in percent, those of trace elements $\left(\mathrm{Ni}, \mathrm{Co}, \mathrm{Cr}, \mathrm{V}\right.$, etc.) in $10^{-4}$ percent. Mineral contents are given as percent of the crystalline phase of the sediment bulk sample.

\section{GEOCHEMISTRY OF SEDIMENTARY SECTIONS}

\section{Hole 379A}

Nine lithological units are distinguished at this site, according to the shipboard data; seven of these are shown on Figures 2 and 3. All are of Pleistocene age. Upper Holocene and middle Holocene sediments studied in piston cores (Figure 4) make up the additional units, with a minimum thickness of 0.5-1.0 meters.

Each unit is correlated with alternating Glacial and Interglacial epochs: Würm (W), Riss-Würm (R-W), Riss (R), Mindel-Riss (M-R), Mindel (M), and GunzMindel (G-M).

Unit 3 (W) basically consists of clay with low calcareous content and some organic matter $\left(\mathrm{CaCO}_{3}\right.$, $12.76-29.97 \%$, and Corg, $0.60-0.70 \%$ ). Only in several interbeds does Corg reach $0.84-0.96 \%$. One of the interbeds appears to be low in iron $(5.04 \% \mathrm{Fe})$. As is shown in the chapter by Trimonis et al. (this volume), clay essentially consists of hydromica, montmorillonite, kaolinite, and chlorite, the first two being main components with hydromica predominating. Beds of terrigenous non-carbonate and low-calcareous sandy aleuritic deposits of different thicknesses occur, sometimes as turbidites.

Geochemical investigations were undertaken principally for clay muds (Tables 2, 3, 4, 5; Figure 2).
$\mathrm{Fe}, \mathrm{Mn}, \mathrm{Ti}, \mathrm{K}, \mathrm{Cu}, \mathrm{Zn}$, and $\mathrm{Pb}$ have a narrow range, whereas the range of $\mathrm{Na}, \mathrm{Ni}, \mathrm{Co}, \mathrm{Li}, \mathrm{Cr}$, and $\mathrm{Zn}$ is broad. A single bed of fine aleuritic ooze in Unit 3 (379A-6-5, 36-46 cm) was also studied. The Corg content in it is only about one-half that of clay oozes, and the contents of $\mathrm{Fe}, \mathrm{Mn}, \mathrm{Cu}, \mathrm{Zn}, \mathrm{Ni}, \mathrm{Cr}$, and $\mathrm{B}$ are low. Conversely, the $\mathrm{Na}$ and particularly $\mathrm{F}$ are high (up to 2400-fold, the maximum concentration at this site).

Units 4 and $5(\mathrm{R}-\mathrm{W})$ mainly consist of clay and silty clay terrigenous (up to 11.76-27.02\%) oozes with markedly increased content of organic matter (up to $0.90 \%$ ). Characteristic components of these units are beds of diatom and nannofossil oozes, enriched in organic matter. Corg ranges from $1.44 \%$ to $2.76 \%$. Contents of $\mathrm{Fe}(3.75-4.95 \%), \mathrm{Mn}(0.07-0.34 \%), \mathrm{K}(1.0-$ $2.06 \%), \mathrm{Na}(0.79-1.91 \%), \mathrm{Cu}(46-83 \mathrm{ppm}), \mathrm{Zn}(77-134$ ppm), Ni (83-168 ppm), Cr (79-118 ppm), V (110-140 ppm), Rb (35-80 ppm), Pb (12-13 ppm), and F (250-440 ppm) range markedly as well. The highest values of $\mathrm{Zn}$, $\mathrm{Pb}$, and $\mathrm{Cs}$ occur in oozes with the highest organic matter content; other elements do not follow this relationship. The highest $\mathrm{Mn}$ content is observed in fine aleuritic oozes poor in organic matter.

Unit $6(\mathrm{R})$ consists of clay and silty clay terrigenous oozes similar in chemical composition to the Unit 3 (W) sediments. They are characterized by relatively low contents of $\mathrm{CaCO}_{3}$ and $\mathrm{Corg}$, and by metal concentrations typical of terrigenous sediments. Only occasionally are the contents of $\mathrm{Fe}$ and $\mathrm{Mn}$ increased (reaching $5.46 \%$ and $0.15 \%$, respectively). In one case the ooze is markedly enriched in Corg (up to $2.04 \%$ ) as well as in $\mathrm{Ni}, \mathrm{Co}, \mathrm{Cr}$, and less so in $\mathrm{Cu}$ and $\mathrm{Zn}$. The average contents of $\mathrm{Ni}, \mathrm{Li}, \mathrm{Rb}, \mathrm{Cs}$, and $\mathrm{B}$ are somewhat increased in the Unit 6 oozes; this is probably related to high illite content.

The Interglacial sediments (Unit 7) are terrigenous, with two beds of sapropel-like sediments containing up to $6 \%$ and $4.50 \%$ of $\mathrm{Corg}$, respectively. $\mathrm{CaCO}_{3}$ content is less than $22 \%$. Only the lowest interbed (Core 29) is highly calcareous (micritic), a result of authigenic calcite accumulation; contents of Corg and various metals are low in this bed. Unit 7 sediments are enriched in $\mathrm{Fe}$ (to $5.55 \%$ ), $\mathrm{Mn}$ (to $0.13 \%$ ), $\mathrm{K}$ (to $2.32 \%$ ), $\mathrm{Cu}, \mathrm{Zn}, \mathrm{Cs}$, etc. (Tables 2, 3). A sample with maximum Corg content $(6 \%)$ is characterized by maximum amounts of $\mathrm{Cu}$ and $\mathrm{Zn}$ and an increased amount of $\mathrm{Ni}$. As a whole, the chemical composition of this unit is nearly the same as that of terrigenous interbeds of other units.

Unit $8(\mathrm{M})$ comprises terrigenous clay and silty clay oozes. They differ from the Unit 7 sediments in their lower contents of $\mathrm{C}_{\text {org }}$ (and $\mathrm{SiO}_{2}$ amorph). However, the unit's upper layers (Cores 40, 39, 36) are appreciably enriched in $\mathrm{Fe}(5.05-7.14 \%)$, and sometimes in $\mathrm{Mn}$ (to $0.15 \%$ ), $\mathrm{K}, \mathrm{Co}$, and $\mathrm{V}$.

The Unit 9 (G-M) sediments at the base of the hole consist of terrigenous muds alternating with calcareous sediments which contain up to $53.8 \% \quad \mathrm{CaCO}_{3}$. Carbonates are basically represented by biogenic calcite; where $\mathrm{CaCO}_{3}$ exceeds $30 \%$, authigenic calcite is present. This calcite and an admixture of authigenic dolomite are characteristic of the sediments within this 


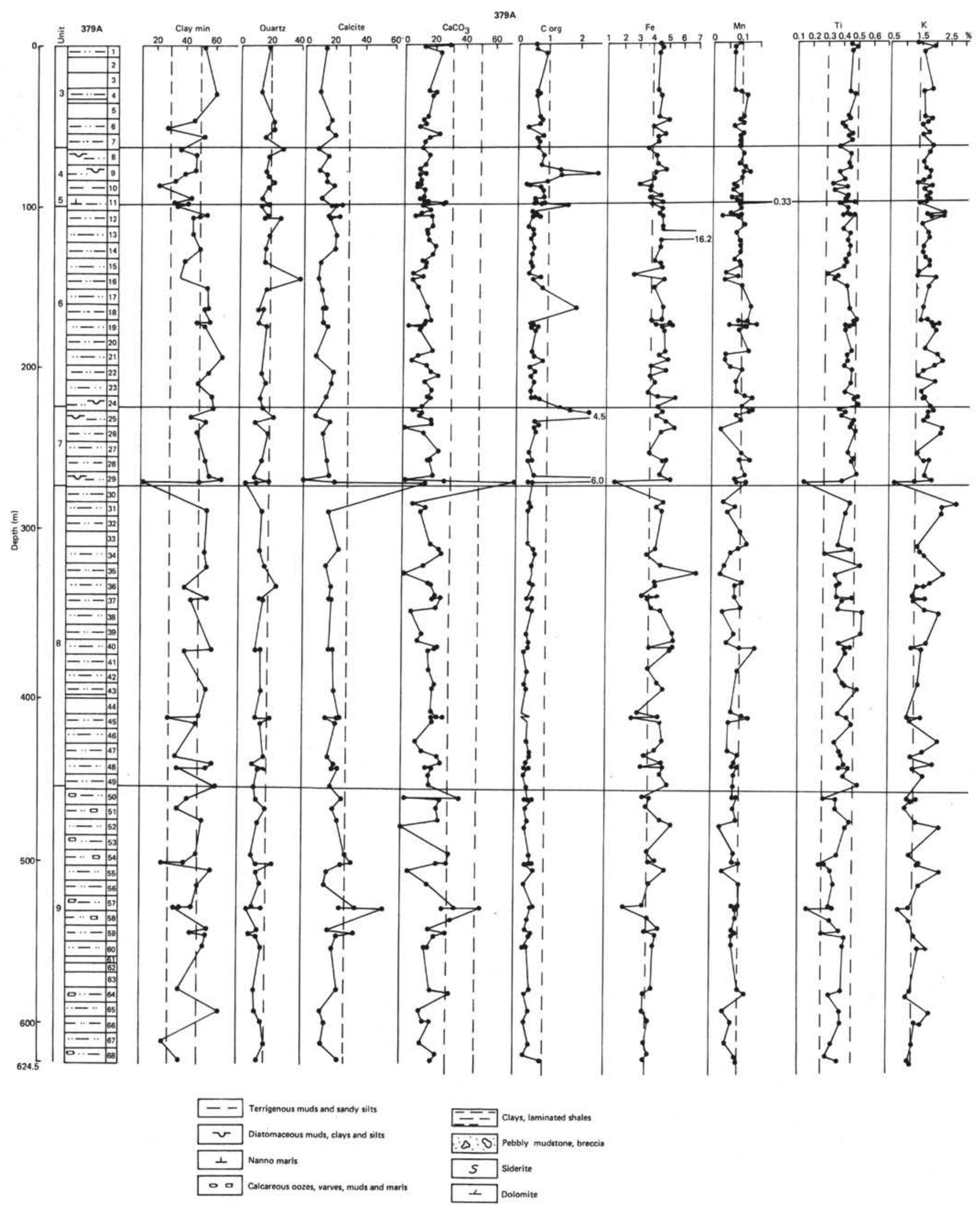

Figure 2. Lithological composition of Hole $379 \mathrm{~A}$ sediments and distribution of main minerals and chemical macrocomponents.

unit, which contain little organic carbon and, probably, $\mathrm{SiO}_{2}$ amorph. The sediments are locally enriched in $\mathrm{Fe}$ (to $5.55 \%$ ) and $\mathrm{Mn}$ (to $0.12 \%$ ). The average contents of other elements are approximately the same as those in 


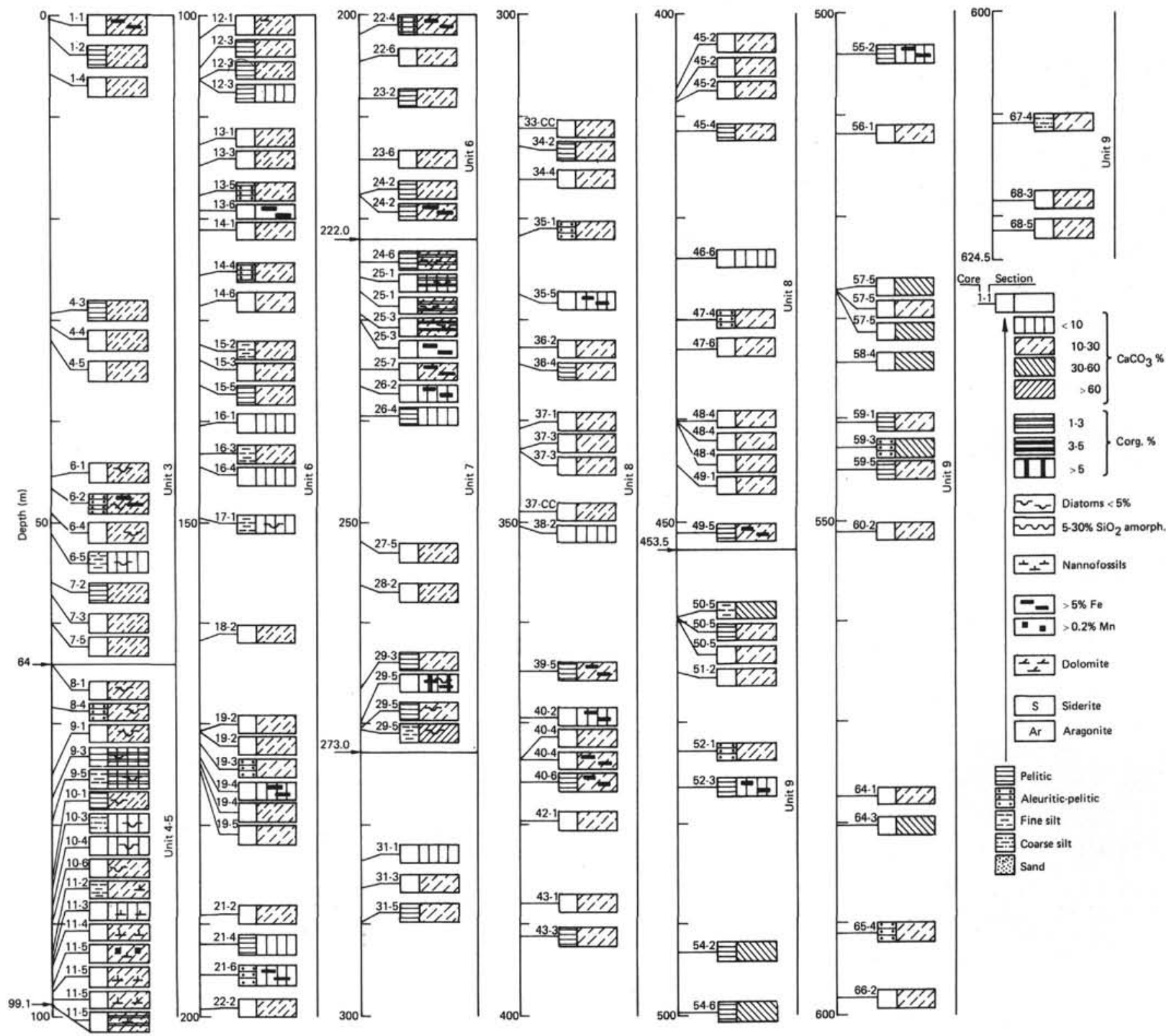

Figure 3. Composition of Hole $379 \mathrm{~A}$ sediment samples.

corresponding types of modern and Pleistocene marine deposits (Tables 6, 7, 8; Figures 5, 6, 7, 8) with the exception of $\mathrm{K}$ and $\mathrm{Co}$, the average contents of which reach the maximum observed at this site. Contents of $\mathrm{FeO}, \mathrm{MgO}$, and occasionally $\mathrm{S}$, are high.

Site 379 sediments are characterized by the following geochemical properties.

1) Units deposited during Glacial epochs basically consist of terrigenous non-calcareous and lowcalcareous clay containing sandy-aleuritic interbeds (turbidites). They also contain diatom and highcalcareous interbeds, as well as sapropelic interbeds more characteristic of the Interglacial sediments (Figures 2, 3).

The above units may be distinguished not only lithologically but geochemically as well. Thus, average contents of $\mathrm{C}_{\mathrm{org}}$ (and $\mathrm{SiO}_{2}$ amorph) are markedly increased in all Interglacial units. Analysis of average contents of various elements (Tables 4,5 ) shows that several units differ in their relative contents of some metals. Thus, $\mathrm{Fe}, \mathrm{Mn}, \mathrm{Cu}, \mathrm{Zn}$, and $\mathrm{Rb}$ are enriched in Unit 7, as are $\mathrm{Co}$ and $\mathrm{V}$ in Unit $8 ; \mathrm{F}, \mathrm{Ni}$, and $\mathrm{Cr}$ in Unit 3; $\mathrm{Ni}, \mathrm{Li}, \mathrm{Rb}, \mathrm{Cs}$, and $\mathrm{B}$ in Unit 6 , etc.

Deposits of Unit 9 (G-M) differ from sediments of the Glacial and Interglacial epochs primarily in the increased content of $\mathrm{CaCO}_{3}$ and decreased content of Corg (Table 4). The $\mathrm{Cr}$ content is also higher. Otherwise, this unit is very similar to Unit $8(\mathrm{M})$.

2) Minor elements have different ranges in the different sediment types, although these differences are minor. In sandy-aleuritic deposits and high-calcareous oozes, the minor element contents are low; in the latter 


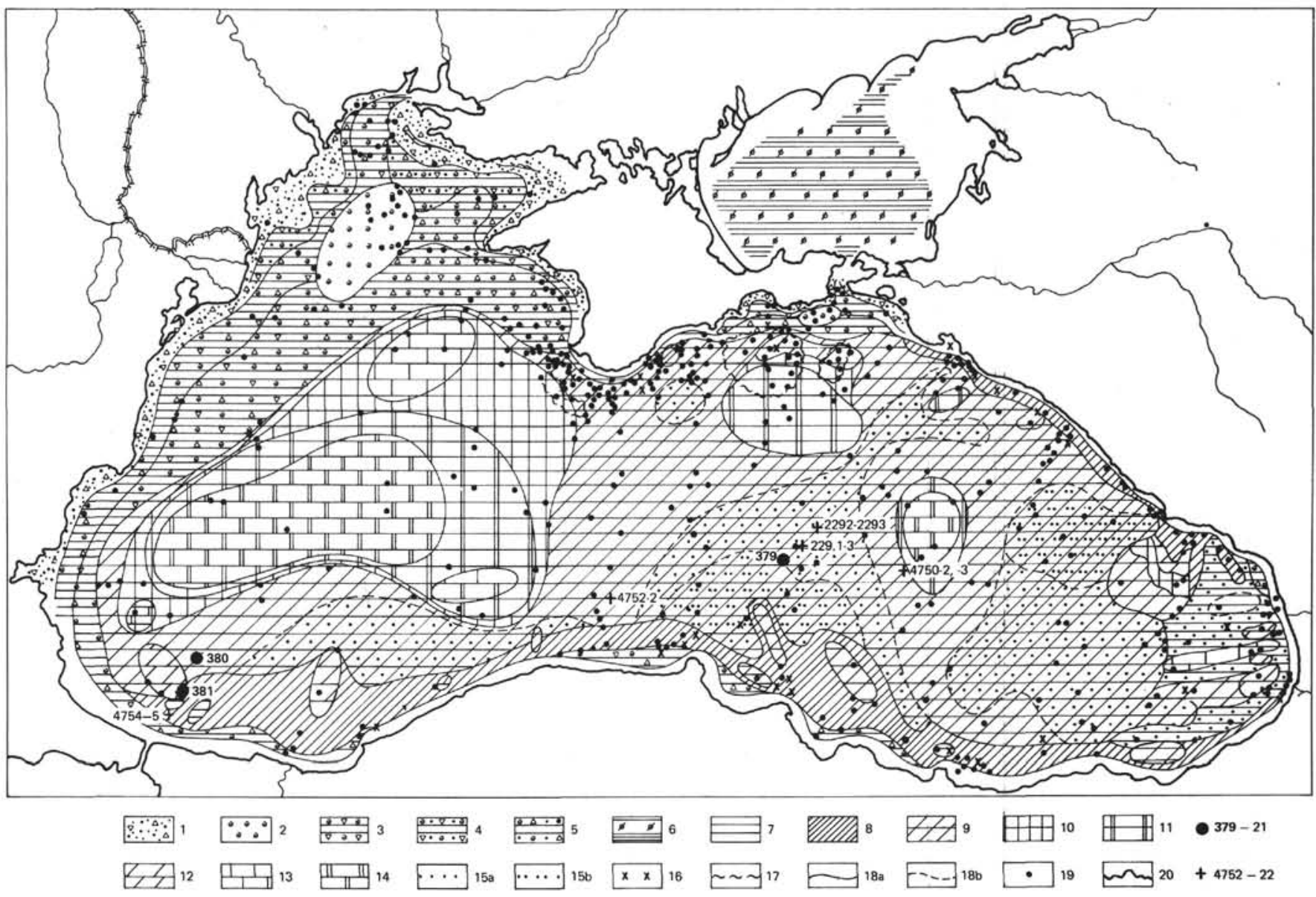

Figure 4. Facies scheme of late Black Sea sediments (upper Holocene, according to Shimkus et al., 1975 a, b). (1) sandaleuritic deposits with a shell detritus admixture; (2) coquinas; (3) low calcareous, calcareous oozes, and high calcareous (more rarely) mytiloid muds (pelitic, aleurite-pelitic) with pure mytiloid coquinas interbeds of small thickness (unit in $\mathrm{cm}$ ); (4) phaseolina low calcareous, calcareous muds (pelitic, aleurite-pelitic) with thin phaseolina coquina interbeds. (5) mytiloid mud interbedding with coastal deposits; (6) terrigenous pelitic muds with coquina interbeds formed by recent Azov fauna; (7) terrigenous pelitic homogeneous low calcareous muds; (8) terrigenous low calcareous muds (pelitic, aleuritepelitic, homogeneous and cryptomicrolaminated); (9) terrigenous low calcareous homogeneous muds with thin calcareous and high calcareous nannofossil microlaminated mud interbeds of less than $5 \mathrm{~cm}$; (10) calcareous nannofossil microlaminated muds with terrigenous low calcareous homogeneous mud; (11) high calcareous nannofossil microlaminated muds with calcareous and terrigenous low calcareous mud interbeds, crypto-, and low-microlaminated; (12) terrigenous low calcareous crypto- and low-microlaminated pelitic and aleurite-pelitic muds; (13) calcareous nannofossil microlaminated muds with high organic matter contents (clay-calcareous mud, according to Strakhov); (14) high calcareous nannofossil muds, microlaminated pelitic enriched in organic matter (calcareous-clay mud according to Strakhov); (15a) aleuritic interbeds of small thickness (less than $5 \mathrm{~cm}$ ), single. (15b) multiple (frequent); (16) regions with late Black Sea sediments absent; (17) distribution boundaries of sediments with disturbed texture (microfolds, uneven interbed boundaries, breccias); (18a) facies boundaries; (19) lithological stations; (20) modern basin boundaries; (21) Sites of Glomar Challenger; (22) stations mentioned in the context. 
TABLE 2

Chemical Composition of Bottom Sediments, Hole 379A

\begin{tabular}{|c|c|c|c|c|c|c|c|c|c|c|c|c|c|c|c|c|c|}
\hline $\begin{array}{c}\text { Depth } \\
\text { in } \\
\text { Hole }\end{array}$ & & & & & Conce & tration & (\%) & & & & & & & & onc & 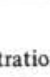 & 11 \\
\hline & (Int & m) & $\mathrm{CaCO}_{3}$ & $\mathrm{C}_{\text {org }}$ & $\mathrm{Fe}$ & $\mathrm{Mn}$ & $\mathrm{Ti}$ & $\mathrm{K}$ & $\mathrm{Na}$ & $\mathrm{Cu}$ & $\mathrm{Zn}$ & $\mathrm{Ni}$ & Co & $\mathrm{Cr}$ & V & $\mathrm{Cd}$ & $\mathrm{Li}$ \\
\hline 0.46 & $1-1$, & $46-48$ & 29.27 & 0.60 & 4.39 & 0.09 & 0.46 & 1.40 & 1.29 & 45 & 82 & 140 & $(19)^{\mathrm{a}}$ & 163 & - & $<6$ & - \\
\hline 2.20 & $1-2$, & $70-78$ & 12.76 & 0.60 & 4.50 & 0.07 & 0.49 & 1.92 & 1.06 & 49 & 77 & 156 & 20 & 156 & 100 & 8 & 14 \\
\hline 5.65 & $1-4$, & $115-125$ & 23.51 & 0.96 & 4.46 & 0.07 & 0.46 & 1.61 & 1.46 & 48 & 85 & 180 & - & 208 & $\overline{-}$ & $<6$ & $\overline{0}$ \\
\hline 29.30 & 4-3, & $30-40$ & 15.26 & 0.63 & 4.30 & .07 & 0.45 & 1.86 & 0.88 & 46 & 87 & 135 & 14 & 196 & 95 & $<5$ & 22 \\
\hline 30.65 & 4-4, & $15-17$ & 20.01 & 0.69 & 4.45 & 0.09 & 0.47 & 1.61 & 0.92 & 51 & 94 & 180 & (13) & 194 & - & $<6$ & - \\
\hline 32.10 & 4-5, & $10-20$ & 18.01 & 0.60 & 4.65 & 0.11 & 0.48 & 1.61 & 0.92 & 51 & 90 & 180 & - & 194 & - & $<6$ & - \\
\hline 45.40 & $6-1$, & $40-42$ & 14.26 & 0.72 & 4.38 & 0.10 & 0.44 & 1.64 & 0.97 & 53 & 91 & 164 & - & 152 & - & $<6$ & - \\
\hline 46.82 & 6-2, & $32-38$ & 11.76 & 0.78 & 5.04 & 0.09 & 0.45 & 1.85 & 0.98 & 54 & 85 & 130 & - & 133 & - & 7 & 16 \\
\hline 49.50 & 6-4, & $0-2$ & 14.26 & 0.69 & 4.45 & 0.10 & 0.40 & 1.71 & 0.99 & 52 & 94 & 120 & - & 152 & - & $<6$ & - \\
\hline 51.36 & 6-5, & $36-46$ & 9.01 & 0.36 & 4.06 & 0.07 & 0.41 & 1.52 & 1.74 & 39 & 70 & 80 & 30 & 79 & 105 & $<6$ & 12 \\
\hline 56.20 & $7-2$, & $20-30$ & 22.76 & 0.84 & 4.80 & 0.10 & 0.46 & 1.74 & 0.85 & 59 & 96 & 140 & 32 & 174 & - & $<5$ & 24 \\
\hline 57.50 & 7-3, & $0-10$ & 15.51 & 0.63 & 4.35 & 0.09 & 0.45 & 1.64 & 0.96 & 51 & 91 & 164 & - & 166 & - & $<6$ & - \\
\hline 60.52 & 7-5, & $2-10$ & 13.51 & 0.69 & 4.35 & 0.09 & 0.46 & 1.22 & 0.92 & 50 & 95 & 156 & - & 166 & - & $<6$ & - \\
\hline Average & & & & 0.68 & 4.48 & 0.09 & 0.45 & 1.64 & 1.07 & 50 & 87 & 148 & 22 & 164 & 100 & $<6$ & 18 \\
\hline 64.09 & 8-1, & $9-11$ & 11.76 & 69 & 3.75 & 0.09 & 0.38 & 2.02 & 1.39 & 57 & 86 & 83 & 32 & 107 & - & 9 & - \\
\hline 68.54 & 8-4, & $4-16$ & 16.51 & 84 & .28 & 0.10 & 0.46 & 1.90 & 0.17 & 46 & 80 & 142 & 40 & 140 & 110 & $<5$ & 14 \\
\hline 74.50 & 9-1, & $100-102$ & 13.26 & 0.78 & 4.27 & 0.09 & 0.44 & 1.88 & 1.26 & 49 & 87 & 165 & - & 123 & - & $<6$ & - \\
\hline 77.50 & 9-3, & $100-108$ & 9.51 & 1.44 & 4.95 & 0.11 & 0.46 & 1.66 & 1.3 & 61 & 94 & 100 & 32 & 94 & - & $<5$ & 22 \\
\hline 80.00 & 9-5, & $50-58$ & 9.76 & 2.76 & 4.19 & 0.12 & 0.37 & 1.76 & 1.08 & 80 & 134 & 114 & - & 144 & - & 10 & 4 \\
\hline 84.20 & 10-1, & $120-130$ & 10.51 & 0.96 & 4.04 & 0.09 & 0.4 & 1.79 & 1. & 4 & 78 & 100 & 43 & 83 & 140 & 7 & 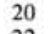 \\
\hline 86.85 & 10-3, & $85-93$ & 10.00 & 0.39 & 3.09 & 0.07 & 0.33 & 1.54 & 1.9 & 40 & 79 & 5 & - & 51 & - & $<6$ & 22 \\
\hline 88.82 & 10-4, & $132-140$ & 9.00 & 0.81 & 3.92 & 0.08 & 0.44 & 2.06 & 1. & 40 & 78 & 92 & - & 108 & - & $<$ & - \\
\hline 90.63 & 10-6, & $13-20$ & 13.01 & 0.87 & 3.84 & 0.07 & 0.35 & 1.58 & 1. & 49 & 77 & 84 & - & 3 & - & & - \\
\hline 94.55 & 11-2, & $55-64$ & 12.01 & 0.90 & 4.60 & 0.09 & 0.44 & 1.44 & 1.41 & 55 & 90 & 84 & 35 & 79 & - & $<5$ & 20 \\
\hline 95.73 & 11-3, & $23-25$ & 9 & 0.57 & 3 & 0.06 & 0.4 & 1.00 & & 2 & 79 & 87 & (15) & 104 & - & 6 & - \\
\hline 98.35 & $11-4$ & $135-143$ & 12. & 0 & 4 & 0. & 0 & 1. & 1 & & 92 & 12 & - & 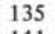 & - & & - \\
\hline 98.68 & 11-5, & 18 & .51 & 0.57 & 4.11 & 0.34 & 0.48 & 1.66 & 1. & 00 & 93 & 147 & - & 141 & - & 2 & - \\
\hline 98.74 & 11-5, & $24-2$ & 27.02 & 0.84 & 4.73 & 0.10 & 0.44 & 1.72 & 0.7 & 83 & 92 & 150 & 50 & 168 & - & 11 & 16 \\
\hline 98.87 & 11-5, & $37-38$ & 26.02 & 0.78 & 4.29 & 0.09 & 0.39 & 1.50 & 0. & 53 & 83 & 168 & - & 157 & - & 7 & - \\
\hline 99.03 & 11-5, & $53-55$ & 16.76 & 1.68 & 4.00 & 0.09 & 0.38 & 1.58 & 1.36 & 53 & 83 & 117 & 41 & 107 & - & $<6$ & 18 \\
\hline Average & & & & 0.97 & 4.12 & 0.10 & 0.41 & 1.69 & 1.27 & 54 & 88 & 113 & 36 & 114 & 125 & $<7$ & 20 \\
\hline 102.37 & $12-1$, & 3 & 17.26 & 0.4 & 68 & .0 & 45 & 1.9 & 0.91 & 54 & 94 & 1 & - & & - & $<6$ & - \\
\hline 105.63 & $12-3$, & $63-72$ & 11.01 & 0.66 & 45 & .06 & 0.44 & 2.28 & 76 & 45 & 91 & 110 & 48 & 122 & - & $<$ & 12 \\
\hline 106.20 & 12-3, & $120-130$ & 18.01 & 0.60 & 4.55 & 0.10 & 0.46 & 1.79 & 0.74 & 46 & 83 & 175 & 40 & 16 & - & $<5$ & 14 \\
\hline 112.70 & 13-1, & $120-128$ & 18.76 & 0.33 & 4.68 & 0.10 & 0.44 & 1.55 & 0.94 & 54 & 82 & 175 & - & 172 & - & 8 & - \\
\hline 114.71 & 13-3, & $21-33$ & 14.76 & 0.54 & 4.68 & 0.09 & 0.42 & 1.66 & 0.92 & 56 & 90 & 167 & - & 161 & - & $<6$ & - \\
\hline 117.51 & 13-5, & $1-7$ & 15.26 & 0.57 & 4.38 & 0.08 & 0.46 & 1.86 & 0.90 & 49 & 87 & 165 & 45 & 153 & - & $<$ & 18 \\
\hline 121.10 & 14-1, & $10-18$ & 16.01 & 0.48 & 4.55 & 0.09 & 0.44 & 1.76 & 1.06 & 50 & 90 & 157 & 21 & 144 & 140 & $<6$ & - \\
\hline 125.50 & 14-4, & 10 & 20.26 & 0.54 & 4.5 & .09 & 0.46 & 1.66 & 3 & 4 & 84 & 170 & 45 & 164 & - & $<$ & 25 \\
\hline 128.52 & 14-6, & $2-4$ & 18.51 & 0.51 & 4.40 & 0.09 & 0.45 & 1.62 & 0.94 & 49 & 90 & 150 & - & 144 & - & $<6$ & - \\
\hline 132.55 & $15-2$, & $55-65$ & 12.01 & 0.51 & 4.18 & 0.07 & 0.43 & 1.65 & 1.31 & 39 & 84 & 108 & 40 & 97 & - & $<5$ & 20 \\
\hline 133.95 & 15-3, & $45-58$ & 14.01 & 0.45 & 4.60 & 0.09 & 0.43 & 1.33 & 0.94 & 50 & 100 & 157 & - & 153 & - & $<6$ & - \\
\hline 136.52 & 15-5, & $2-12$ & 13.76 & 0.51 & 4.68 & 0.09 & 0.42 & 1.76 & 1.09 & 49 & 90 & 136 & - & 115 & - & $<$ & - \\
\hline 140.30 & 16-1, & $30-38$ & 5 & 0.42 & 2.77 & 0.04 & 0.31 & 1.62 & 0. & 36 & 63 & 95 & $\overline{-}$ & 8 & - & $<6$ & $\bar{n}$ \\
\hline 143.17 & 16-3, & $17-27$ & 12.51 & o & 3.95 & 9 & 0 & 1. & 2 & 40 & 66 & 142 & 35 & 128 & - & $<$ & 24 \\
\hline 144.67 & $16-4$, & $17-19$ & 5.26 & 0.54 & 4.86 & .04 & 0.37 & 2. & 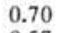 & 47 & 107 & 16 & $\overline{-}$ & 1 & - & $<6$ & $\bar{x}$ \\
\hline 149.75 & 17-1, & $25-35$ & 9.01 & 0.84 & 4.20 & .10 & 0.44 & 1.80 & 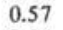 & 61 & 102 & 132 & 45 & 115 & 120 & $<$ & 35 \\
\hline 161.78 & 18-2, & $128-130$ & 15.01 & 2.04 & 4.78 & 0.13 & 0.45 & 1.62 & 0.94 & 79 & 102 & 206 & 50 & 190 & - & $<6$ & 25 \\
\hline 170.76 & 19-2, & $76-78$ & 17.01 & 0.54 & 4.68 & 0.11 & 0.50 & 1.64 & 0.92 & 50 & 92 & 168 & 36 & 175 & - & $<6$ & 25 \\
\hline 170.80 & 19-2, & $80-82$ & 14.51 & 0 & 4.00 & .08 & 9 & 1.80 & 1. & 50 & 94 & 182 & 37 & 1 & - & 8 & 19 \\
\hline 172.32 & 19-3, & $82-98$ & 13.51 & 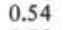 & 4.30 & 0.15 & 0.49 & 2.03 & 0. & 51 & 99 & 1 & 49 & 176 & - & 7 & 35 \\
\hline 173.57 & 19-4, & $57-59$ & 3.25 & .72 & 5.46 & .05 & 0.43 & 2.30 & 0.7 & 54 & 111 & 12 & - & 13 & - & $<6$ & - \\
\hline 173.66 & 19-4, & $66-68$ & 11.01 & 0.51 & 4.76 & .11 & 0.46 & 1.79 & 0.86 & 66 & 99 & 20 & - & 18 & - & 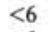 & - \\
\hline 175.70 & 19-5, & $120-132$ & 10.51 & 0.63 & 4.85 & 0.09 & 0.43 & 1.94 & 0. & 58 & 98 & 161 & 36 & 140 & 240 & $<6$ & - \\
\hline 189.20 & 21-2, & $20-29$ & 19.01 & 0.51 & 4.90 & 0.12 & 0.47 & 1.75 & 0 & 55 & 92 & 184 & - & 160 & - & $<6$ & - \\
\hline 192.00 & $21-4$, & $a$ & 0 & 77 & 4 & 4 & 4 & 2.08 & 9 & 53 & 112 & 150 & 40 & 9 & - & $<5$ & 28 \\
\hline 195.00 & $21-6$, & 0 & 4.50 & 17 & 5 & 4 & 4 & 2.24 & 0 & 6 & 105 & 1 & - & 3 & - & 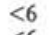 & - \\
\hline 198.50 & $22-2$, & $0-10$ & 15.26 & 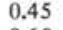 & 3 & 0.06 & 0 & .06 & 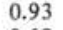 & 45 & 89 & 10 & - & 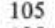 & - & $<$ & - \\
\hline 201.97 & $22-4$ & $47-55$ & 17.76 & 60 & 5.02 & 1 & 0.47 & 1.59 & 0.6 & 44 & 104 & 20 & 42 & 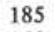 & - & $<5$ & 30 \\
\hline 204.50 & $22-6$ & $0-10$ & 22.76 & 0.48 & 4.00 & 0.09 & 0.47 & 1.41 & 0.98 & 45 & 80 & 168 & $\overline{-}$ & 143 & - & $<6$ & - \\
\hline 208.00 & $23-2$, & $0-11$ & 13.76 & 0.57 & 4.20 & 0.08 & 0.47 & 2.08 & 0.62 & 54 & 94 & 147 & 37 & 163 & 160 & $<5$ & 28 \\
\hline 214.00 & 23-6, & $0-17$ & 18.76 & 0.51 & 3.80 & 0.08 & 0.43 & 1.49 & 1.16 & 46 & 76 & 142 & - & 148 & - & $<6$ & - \\
\hline 217.50 & $24-2$ & $0-18$ & 18.01 & 0.63 & 4.46 & 0.11 & 0.51 & 1.66 & 0.77 & 52 & 100 & 180 & 52 & 160 & - & 8 & 20 \\
\hline Average & & & & 0.61 & 4.47 & 0.08 & 0.44 & 1.79 & 0.88 & 51 & 92 & 15 & 41 & 150 & 165 & $<6$ & 24 \\
\hline & & & 2.0 & 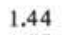 & .3 & & & 10 & & 5 & 92 & & 4 & & 170 & - & 25 \\
\hline & -1 , & 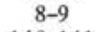 & & & - & & & & & & 5 & & - & 98 & - & $<6$ & - \\
\hline 226.90 & 25-1, & $140-141$ & 12.01 & 2.49 & 4.74 & 0.12 & 0.42 & 1.85 & ( & 56 & 102 & 172 & 43 & 145 & - & $<6$ & - \\
\hline 228.92 & $25-3$, & $42-44$ & 11.26 & 4.50 & 4.43 & 0.08 & 0.41 & 1.83 & 1.04 & 72 & 102 & 120 & 37 & 77 & - & 10 & - \\
\hline 232.45 & $25-5$ & $95-106$ & - & - & - & - & - & - & - & - & - & - & - & - & - & - & 25 \\
\hline 234.79 & $25-7$, & $29-31$ & 19.01 & 0.72 & 5.10 & 0.10 & 0.48 & 1.58 & & 56 & 93 & 164 & - & 190 & - & $<6$ & - \\
\hline .63 & $26-2$, & $13-15$ & 0.50 & & & 0 & & & & 55 & 116 & 9 & - & & - & $<6$ & - \\
\hline 50 & $26-4$, & 0 & & c & 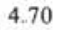 & .04 & 0 & 0 & & 4 & 98 & 17 & 47 & 142 & 150 & 8 & 35 \\
\hline 251.97 & 27-5, & $147-149$ & 23.26 & 0.42 & 3.96 & 0.10 & 0.45 & 1.48 & 1. & 40 & 73 & 135 & - & 150 & - & $<6$ & - \\
\hline 256.32 & $28-2$, & $82-83$ & 15.76 & 0.54 & 4.95 & 0.09 & 0.47 & 1.76 & 0.82 & 59 & 116 & 189 & - & 201 & - & $<6$ & 14 \\
\hline 266.50 & 29-3, & $0-14$ & 20.26 & 0.57 & 4.60 & 0.11 & 0.50 & 1.64 & 0.72 & 51 & 84 & 167 & 40 & 210 & - & $<5$ & 24 \\
\hline 269.54 & 29-5, & $4-6$ & 1.00 & 6.00 & 5.30 & 0.08 & - & 1.99 & 0.62 & 128 & 128 & 168 & (36) & 116 & - & $<6$ & - \\
\hline 48 & 29-5, & $98-10$ & 26.52 & 0.39 & 3.75 & 0.08 & 0.41 & 1.29 & 0.98 & 33 & 62 & 138 & 46 & 176 & 120 & $<5$ & 20 \\
\hline 270.57 & 29-5, & $107-112$ & 73.80 & 0.54 & 1.50 & 0.12 & 0.16 & 0.58 & 0.34 & 32 & 37 & 52 & - & 44 & - & $<6$ & 8 \\
\hline Average & & & & 1.59 & 4.42 & 0.09 & 0.43 & 1.73 & 0.76 & 57 & 93 & 136 & 42 & 140 & 147 & $<6$ & 21 \\
\hline
\end{tabular}


TABLE 2 - Continued

\begin{tabular}{|c|c|c|c|c|c|c|c|c|c|c|c|c|c|c|c|c|c|c|c|c|c|c|c|}
\hline \multirow{2}{*}{$\begin{array}{c}\text { Depth } \\
\text { in } \\
\text { Hole } \\
\text { (m) }\end{array}$} & $\mathrm{Sa}$ & & & & Concen & ration & & & & & & & & & oncent & ation & $\left(10^{-4}\right.$ & $\%)$ & & & & & i \\
\hline & & & $\mathrm{CaCO}_{3}$ & $\mathrm{C}_{\text {org }}$ & $\mathrm{Fe}$ & $\mathrm{Mn}$ & $\mathrm{Ti}$ & K & $\mathrm{Na}$ & $\mathrm{Cu}$ & $\mathrm{Zn}$ & $\mathrm{Ni}$ & Co & $\mathrm{Cr}$ & V & $\mathrm{Cd}$ & $\mathrm{Li}$ & $\mathrm{Rb}$ & Cs & $\mathrm{Pb}$ & $\mathrm{Sn}$ & $\mathrm{F}$ & B \\
\hline 283.70 & $31-1$ & $120-122$ & 7.25 & 0.45 & 4.93 & 0.04 & 0.47 & 2.92 & 0.50 & 44 & 86 & 100 & - & 121 & - & $<6$ & - & - & - & - & - & - & - \\
\hline 286.56 & $31-3$ & $106-108$ & 15.26 & 0.51 & 4.44 & 0.08 & 0.45 & 2.22 & 0.71 & 40 & 103 & 109 & - & 127 & - & $<6$ & - & - & - & - & - & - & - \\
\hline 289.70 & $31-5$ & $120-132$ & 12.26 & 0.45 & 4.80 & 0.06 & 0.43 & 2.33 & 0.58 & 44 & 90 & 132 & 53 & 153 & 170 & $<5$ & 22 & 50 & 4.5 & 20 & 2.0 & 920 & 139 \\
\hline 311.00 & $33, \mathrm{CC}$ & & 18.26 & 0.42 & 4.50 & 0.12 & 0.39 & 1.54 & 0.80 & 46 & 79 & 155 & - & 164 & - & $<6$ & - & - & - & - & - & - & - \\
\hline 312.88 & $34-2$ & $38-50$ & 23.76 & 0.60 & 4.40 & 0.09 & 0.48 & 1.56 & 0.73 & 45 & 84 & 168 & 43 & 161 & - & 7 & 25 & 66 & 5.0 & 6 & 1.2 & - & - \\
\hline 316.19 & $34-4$ & $69-71$ & 26.02 & 0.66 & 3.90 & 0.07 & 0.31 & 1.80 & 0.59 & 56 & 97 & 91 & $(10)$ & 104 & - & $<6$ & - & $\overline{-}$ & - & - & - & - & - \\
\hline 321.81 & $35-1$ & $131-141$ & 13.76 & 0.57 & 4.72 & 0.05 & 0.54 & - & - & 51 & 88 & 152 & 40 & 183 & - & 8 & 25 & 56 & 5.0 & 15 & 1.3 & - & - \\
\hline 327.42 & $35-5$ & $92-101$ & 1.50 & 0.57 & 7.14 & 0.03 & 0.37 & 2.32 & 0.64 & 60 & 116 & 119 & - & 115 & - & $<6$ & - & - & - & - & - & - & - \\
\hline 332.87 & $36-2$ & $137-145$ & 17.26 & 0.51 & 4.28 & 0.11 & 0.40 & 1.90 & 0.74 & 36 & 97 & 107 & 18 & 114 & 120 & $<6$ & - & - & - & - & - & 680 & - \\
\hline 334.59 & $36-4$ & $9-23$ & 18.51 & 0.60 & 4.38 & 0.08 & 0.38 & 1.70 & 0.94 & 44 & 84 & 145 & - & 166 & - & $<6$ & 24 & 70 & 5.6 & 18 & 1.5 & - & - \\
\hline 340.76 & $37-1$ & $126-135$ & 22.01 & 0.48 & 3.52 & 0.08 & 0.38 & 1.41 & 1.06 & 34 & 74 & 128 & - & 134 & - & $<6$ & - & - & - & - & - & - & - \\
\hline 342.78 & $37-3$ & $28-30$ & 20.26 & 0.42 & 4.60 & 0.08 & 0.49 & 1.68 & 0.72 & 49 & 94 & 167 & - & 197 & - & $<6$ & 19 & 48 & 4.0 & 30 & 2.5 & - & - \\
\hline 342.83 & $37-3$ & $33-35$ & 25.27 & 0.57 & 3.90 & 0.08 & 0.41 & 1.46 & 1.18 & 39 & 71 & 155 & - & 176 & - & $<6$ & 27 & 60 & 5.2 & 10 & 1.2 & - & - \\
\hline 349.00 & $37, \mathrm{CC}$ & & 22.76 & 0.45 & 4.18 & 0.10 & 0.38 & 1.96 & 0.78 & 40 & 81 & 156 & - & 146 & - & $<6$ & - & - & - & - & - & - & - \\
\hline 350.61 & $38-2$ & $11-13$ & 6.00 & 0.60 & 4.75 & 0.04 & 0.54 & 2.47 & 0.46 & 50 & 102 & 128 & (17) & 113 & - & $<6$ & - & - & - & - & - & - & - \\
\hline 364.92 & $39-5$ & $42-54$ & 13.01 & 0.42 & 5.54 & 0.08 & 0.54 & 1.82 & 0.68 & 50 & 100 & 178 & - & 170 & - & $<6$ & - & - & - & - & - & - & - \\
\hline 369.64 & $40-2$ & $14-24$ & 9.76 & 0.48 & 5.55 & 0.05 & 0.40 & 2.16 & 0.54 & 47 & 96 & 132 & - & 136 & - & $<6$ & - & - & - & - & - & - & - \\
\hline 373.90 & $40-4$ & $140-142$ & 23.01 & 0.45 & 3.95 & 0.09 & 0.47 & 1.34 & 1.16 & 41 & 73 & 166 & (19) & 200 & - & $<6$ & 27 & 70 & 5.6 & 10 & 1.4 & - & - \\
\hline 373.94 & $40-4$ & $144-146$ & 21.76 & 0.42 & 5.05 & 0.15 & 0.45 & 1.69 & 0.64 & 51 & 86 & 170 & (17) & 206 & - & $<6$ & 28 & 56 & 5.0 & 10 & 1.4 & - & - \\
\hline 376.18 & $40-6$ & $58-76$ & 17.01 & 0.33 & 5.37 & 0.14 & 0.44 & 1.71 & 0.72 & 52 & 85 & 185 & - & 176 & - & $<6$ & - & - & - & - & - & - & - \\
\hline 379.75 & $42-1$ & $25-37$ & 18.01 & 0.42 & 3.85 & 0.09 & 0.38 & 1.54 & 0.88 & 47 & 79 & 130 & - & 133 & - & $<6$ & - & - & - & - & - & - & - \\
\hline 388.25 & $43-1$ & $125-135$ & 21.76 & 0.46 & 4.47 & 0.08 & 0.43 & 1.54 & 0.81 & 55 & 82 & 162 & 24 & 154 & 170 & $<6$ & - & - & - & $\overline{-}$ & - & 600 & 151 \\
\hline 391.40 & $43-3$ & $140-150$ & 20.76 & 0.39 & 4.96 & 0.08 & 0.52 & - & - & 42 & 84 & 172 & 40 & 190 & - & $<8$ & 28 & 66 & 5.0 & 10 & 1.0 & - & - \\
\hline 407.60 & $45-2$ & $10-12$ & 19.01 & 0.27 & 3.11 & 0.07 & 0.39 & 1.15 & 1.36 & 24 & 51 & 113 & - & 120 & - & $<6$ & - & - & - & - & - & - & - \\
\hline 408.58 & $45-2$ & $108-110$ & 23.01 & 0.51 & 4.63 & 0.11 & 0.45 & 1.69 & 0.65 & 46 & 87 & 168 & - & 171 & - & $<6$ & 25 & 60 & 5.6 & 10 & 1.0 & - & - \\
\hline 408.62 & $45-2$ & $112-114$ & - & - & - & - & - & - & - & - & - & - & - & - & - & - & 27 & 70 & 5.5 & 8 & 1.0 & - & - \\
\hline 408.66 & $45-2$ & $116-118$ & 20.51 & 0.30 & 2.84 & 0.13 & - & 1.17 & 1.44 & 26 & 58 & 142 & - & 164 & - & $<6$ & 17 & 35 & 2.5 & 20 & 1.2 & - & - \\
\hline 411.48 & $45-4$ & $98-106$ & 20.26 & 0.45 & 4.70 & 0.06 & 0.49 & - & - & 40 & 84 & 163 & 36 & 183 & - & 8 & 20 & 60 & 5.0 & 12 & 1.2 & - & - \\
\hline 424.02 & $46-6$ & $102-116$ & 9.26 & 0.42 & 4.94 & 0.06 & 0.37 & 2.15 & 0.66 & 67 & 115 & 120 & - & 123 & - & $<6$ & - & - & - & - & - & - & - \\
\hline 430.08 & $47-4$ & $58-74$ & 13.76 & 0.51 & 4.33 & 0.06 & 0.40 & 1.68 & 1.02 & 45 & 74 & 95 & - & 137 & - & $<6$ & - & - & - & - & - & - & - \\
\hline 433.15 & $47-6$ & $65-67$ & 24.26 & 0.51 & 3.51 & 0.09 & 0.42 & 1.20 & 1.29 & 38 & 64 & 147 & - & 178 & - & $<6$ & 20 & 45 & 4.5 & 10 & 1.0 & - & - \\
\hline 437.27 & $48-2$ & $127-135$ & - & - & - & - & - & - & - & - & - & 23 & 33 & 240 & 190 & - & 24 & 50 & 4.5 & 7 & 1.2 & 660 & - \\
\hline 440.25 & $48-4$ & $125-127$ & 16.26 & 0.45 & 4.86 & 0.08 & 0.44 & 1.98 & 0.50 & 52 & 96 & 151 & - & 113 & - & $<6$ & 22 & 52 & 5.0 & 16 & 1.2 & - & - \\
\hline 440.28 & $48-4$ & $128-130$ & 19.26 & 0.36 & 3.35 & 0.09 & 0.39 & 1.31 & 1.27 & 39 & 66 & 148 & - & 181 & - & $<6$ & 22 & 40 & 2.0 & 12 & 1.0 & - & - \\
\hline 440.32 & $48-4$ & $132-140$ & 20.51 & 0.45 & 4.90 & 0.08 & 0.47 & - & - & 38 & 84 & 189 & 38 & 226 & - & 8 & 28 & 50 & 5.8 & 10 & 1.0 & - & - \\
\hline 444.28 & 49-1, & $28-41$ & 18.76 & 0.33 & 4.85 & 0.08 & 0.43 & 1.63 & 0.61 & 47 & 81 & 115 & - & 156 & - & $<6$ & - & - & - & - & - & - & - \\
\hline 451.22 & 49-5, & $122-130$ & 19.01 & 0.42 & 5.20 & 0.08 & 0.52 & - & - & 46 & 84 & 186 & 40 & 203 & - & 8 & 28 & 70 & 6.0 & 10 & 1.1 & - & - \\
\hline Average & & & & 0.46 & 4.52 & 0.08 & 0.44 & 1.77 & 0.82 & 45 & 85 & 141 & 31 & 159 & 162 & $<6$ & 24 & 57 & 4.8 & 13 & 1.3 & 715 & 145 \\
\hline 459.62 & $50-5$ & $12-21$ & 38.78 & 0.48 & 3.59 & 0.08 & 0.29 & 1.17 & 0.82 & 40 & 64 & 72 & - & 105 & - & $<6$ & - & - & - & - & - & - & - \\
\hline 459.62 & $50-5$, & $12-24$ & 26.27 & 0.63 & 4.05 & 0.09 & 0.38 & 1.30 & 0.85 & 45 & 100 & 168 & - & 166 & - & $<6$ & 20 & 50 & 4.5 & 10 & 1.0 & - & - \\
\hline 465.14 & $51-2$ & $64-66$ & 23.52 & 0.39 & 2.85 & 0.08 & 0.38 & 1.10 & 1.29 & 30 & 61 & 132 & - & 149 & - & $<6$ & 20 & 50 & 4.0 & 8 & 1.0 & - & - \\
\hline 473.05 & $52-1$ & $55-65$ & 25.52 & 0.45 & 4.80 & 0.09 & 0.47 & 1.50 & 0.82 & 58 & 78 & 192 & 30 & 301 & 170 & $<5$ & 30 & 54 & 5.2 & 12 & 1.5 & 590 & 100 \\
\hline 476.55 & $52-3$ & $105-114$ & 0.00 & 0.36 & 5.55 & 0.03 & 0.44 & 2.19 & 0.59 & 68 & 107 & 95 & (21) & 142 & - & $<6$ & - & - & - & - & - & - & - \\
\hline 493.20 & $54-2$, & $20-34$ & 32.52 & 0.54 & 3.85 & 0.08 & 0.40 & 1.29 & 0.71 & 39 & 68 & 110 & 47 & 155 & 130 & $<5$ & 19 & 35 & 4.0 & 10 & 1.0 & 390 & - \\
\hline 499.78 & $54-6$ & $78-85$ & 31.52 & 0.54 & 4.04 & 0.07 & 0.28 & 1.54 & 0.56 & 53 & 78 & 73 & - & 108 & - & $<6$ & - & - & - & - & - & - & - \\
\hline 499.78 & $54-6$ & $78-86$ & 31.02 & 0.60 & 4.02 & 0.10 & 0.31 & 1.46 & 0.59 & 37 & 78 & 126 & - & 130 & - & $<6$ & 18 & 56 & 5.0 & 12 & 1.2 & - & - \\
\hline 499.85 & $54-6$ & $85-88$ & - & - & - & - & - & - & - & - & - & - & - & - & - & - & 16 & 35 & 3.5 & 10 & 1.1 & - & - \\
\hline 503.93 & $55-2$ & $143-145$ & 5.0 & 0.66 & 5.10 & 0.04 & 0.36 & 2.33 & 0.74 & 67 & 125 & 114 & - & 174 & - & $<6$ & 17 & 70 & 6.0 & 14 & 1.4 & - & - \\
\hline 511.83 & $56-1$, & $133-143$ & 18.26 & 0.36 & 4.03 & 0.10 & 0.38 & 1.60 & 0.93 & 54 & 100 & 134 & (15) & 170 & - & $<6$ & 24 & 60 & 6.0 & 16 & 1.4 & - & - \\
\hline 527.24 & $57-5$ & $124-126$ & 36.27 & 0.66 & 3.76 & 0.10 & 0.34 & 1.29 & 0.59 & 44 & 97 & 134 & - & 180 & - & $<6$ & 16 & 54 & 5.6 & 10 & 1.1 & - & - \\
\hline 527.27 & $57-5$ & $127-129$ & 28.02 & 0.60 & 3.10 & 0.08 & 0.38 & 1.17 & 1.23 & 38 & 73 & 138 & - & 168 & - & $<6$ & 16 & 50 & 4.5 & 10 & 1.0 & - & - \\
\hline 527.30 & $57-5$ & $130-132$ & 53.78 & 0.60 & 2.26 & 0.09 & 0.20 & 0.94 & 0.46 & 61 & 65 & 81 & - & 76 & - & $<6$ & 10 & 30 & 3.5 & 5 & 1.0 & - & - \\
\hline 534.53 & $58-4$ & $53-61$ & 33.76 & 0.51 & 4.00 & 0.09 & 0.36 & 1.34 & 0.65 & 42 & 58 & 50 & - & 116 & - & $<6$ & - & - & - & - & - & 480 & - \\
\hline 540.48 & 59-1, & $148-150$ & 19.01 & 0.39 & 4.67 & 0.08 & 0.42 & - & - & 40 & 78 & 174 & 40 & 200 & - & 8 & 30 & 70 & 5.6 & 14 & 1.2 & - & - \\
\hline 542.90 & 59-3, & $90-100$ & 30.52 & 0.63 & 3.88 & 0.09 & 0.31 & 1.44 & 0.60 & 46 & 80 & 135 & - & 142 & - & $<6$ & 18 & 50 & 4.5 & 10 & 1.1 & - & - \\
\hline 545.05 & $59-5$ & $5-13$ & 22.76 & 0.54 & 4.64 & 0.08 & 0.46 & - & - & 46 & 76 & 136 & 35 & 185 & - & 7 & 30 & 56 & 6.8 & 15 & 1.2 & - & - \\
\hline 551.35 & $60-2$ & $135-145$ & 16.76 & 0.45 & 4.37 & 0.08 & 0.44 & 1.82 & 0.68 & 59 & 107 & 146 & (19) & 176 & - & $<6$ & 16 & 60 & 5.6 & 12 & 1.0 & 570 & 119 \\
\hline 577.75 & 64-1, & $75-77$ & 20.01 & 0.60 & 4.29 & 0.10 & 0.44 & 1.30 & 1.09 & 54 & 77 & 134 & - & 174 & - & $<6$ & 16 & 45 & 3.5 & 10 & 1.0 & - & - \\
\hline 580.57 & $64-3$ & $57-59$ & 33.52 & 0.42 & 3.84 & 0.12 & 0.33 & 1.20 & 0.88 & 39 & 68 & 95 & - & 140 & - & $<6$ & - & - & - & - & - & - & - \\
\hline 591.37 & $65-4$ & $37-49$ & 12.76 & 0.54 & 3.70 & 0.05 & 0.43 & 1.99 & 0.60 & 48 & 104 & 167 & 30 & 188 & 180 & $<5$ & 30 & 60 & 6.8 & 16 & 1.2 & 560 & 87 \\
\hline 597.96 & $66-2$ & $48-58$ & 15.31 & 0.39 & 4.09 & 0.08 & 0.44 & 1.69 & 0.74 & 58 & 93 & 164 & 25 & 178 & 120 & $<6$ & 20 & 60 & 5.2 & 12 & 1.2 & - & - \\
\hline 611.10 & $67-4$ & $110-120$ & 14.44 & 0.54 & 3.95 & 0.06 & 0.38 & 1.46 & 1.56 & 35 & 62 & 128 & 38 & 184 & - & $<5$ & 17 & 40 & 3.0 & 10 & 1.0 & - & - \\
\hline 618.66 & $68-3$ & $66-68$ & 25.27 & 0.39 & 4.08 & 0.09 & 0.34 & 1.29 & 1.11 & 36 & 74 & 95 & 23 & 140 & - & $<6$ & - & - & - & - & - & - & - \\
\hline 621.73 & $68-5$ & $73-75$ & 15.51 & 0.96 & 3.86 & 0.10 & 0.42 & 1.32 & 1.10 & 57 & 90 & 137 & 19 & 156 & - & $<6$ & 16 & 50 & 4.5 & 10 & 1.1 & - & - \\
\hline Average & & & & 0.53 & 4.01 & 0.08 & 0.38 & 1.47 & 0.83 & 48 & 82 & 125 & 28 & 160 & 150 & $<6$ & 20 & 52 & 4.9 & 11 & 1.1 & 518 & 102 \\
\hline Averag & ent & & & & & & & & & & & & & & & & & & & & & & \\
\hline $\begin{array}{l}\text { Terrigen } \\
\text { (Units 3, }\end{array}$ & $\begin{array}{c}\text { ous mud } \\
6,8,9)\end{array}$ & $s(<30 \%$ C & & 0.54 & 4.45 & 0.08 & 0.43 & 1.78 & 0.90 & 48 & 88 & 147 & 32 & 159 & 148 & $<6$ & 23 & 56 & 4.4 & 14 & 1.4 & 641 & 116 \\
\hline Diatomac & ceous oo & zes (Units & $4,7)$ & 1.60 & 4.48 & 0.10 & 0.44 & 1.76 & 1.13 & 57 & 94 & 130 & 44 & 132 & 138 & 7 & 23 & 59 & 4.8 & 20 & 1.6 & 482 & 100 \\
\hline Coccolith & muds ( & $>30 \% \mathrm{Ca}$ & $\left.\mathrm{O}_{3}\right)$ & 0.55 & 3.32 & 0.09 & 0.29 & 1.23 & 0.62 & 43 & 70 & 92 & 47 & 119 & 130 & $<6$ & 15 & 41 & 2.8 & 8 & 1.1 & 390 & - \\
\hline Average & in Hole & & 17.63 & 0.69 & 4.34 & 0.09 & 0.42 & 1.68 & 0.91 & 50 & 88 & 139 & 34 & 152 & 145 & $<6$ & 22 & 54 & 4.7 & 15 & 1.4 & 590 & 111 \\
\hline
\end{tabular}

a(19) - data of INAA.

case this is due to dilution by carbonate. Oozes with high organic matter content are sometimes slightly enriched in $\mathrm{Ni}, \mathrm{Co}, \mathrm{Cr}, \mathrm{Cu}$, and $\mathrm{Zn}$.

3) Average contents of the following elements from Site 379 sediments have the largest ranges: (a) $\mathrm{Ni}-$ from 118 to $156 \mathrm{ppm}$; the higher contents are often observed in the Glacial sediments; (b) $\mathrm{Cr}-$ from 110 to $175 \mathrm{ppm}$, decreased in Interglacial sediments; (c) V-from 100 to $162 \mathrm{ppm}$, with some increase in the lower part of the section; (d) $\mathrm{Fe}$-from 343 to 1388 
ppm, the maximum in Unit 3 deposits (W); and (e) B-from 89 to $154 \mathrm{ppm}$, with higher values in Units 6 and 8 ( $R$ and $M)$.

4) Ratios of average contents of the studied elements vary considerably from their clarkes (Tables $4,5,9$ ).

In all units, $\mathrm{Fe}, \mathrm{Mn}, \mathrm{Na}, \mathrm{Ni}, \mathrm{Co}, \mathrm{Cr}$, and $\mathrm{Sc}$ contents are higher than their clarkes in sedimentary rocks $(\mathrm{Ko})$, with $\mathrm{Ni}, \mathrm{Co}, \mathrm{Cr}$, and $\mathrm{Sc}$ contents throughout the section being about 1.5-2 times higher than Ko and close to their clarkes in basic rocks (Kb) (Table 9). Throughout the section, $\mathrm{K}, \mathrm{Li}, \mathrm{Rb}, \mathrm{Cs}$, and $\mathrm{Sn}$, i.e., the elements from acid volcanic and sedimentary rocks, are lower than Ko (Table 9).

5) Average contents of the elements studied and the ratios to their clarkes show the importance of basic, ultrabasic, and intermediate rock weathering products in Pleistocene sediments. These are widely distributed in the Pontic drainage system and are eroded by the largest rivers such as the Kizyl-Irmak and (to a greater extent) the Eshil-Irmak. The Kizyl-Irmak River erodes Pre-Cambrian crystalline rocks of intermediate and acid composition, as well as basic-ultrabasic intrusive and ophiolite series.

\section{Site 380}

Based on shipboard analysis, seven units were distinguished, representing the Glacial and Interglacial epochs W, R-W, R, M-R, and M (see above). The lower part of the site is represented by Eopleistocene (Eopleistocene corresponds to Menap, Waal, and Elwon) (Figures 9, 10). Main lithological differences between these units were confirmed by shore-based analyses.

Unit B-1-c (W) is represented mainly by terrigenous clay and silty clay mud with aleuritic and sandy interbeds. $\mathrm{CaCO}_{3}$ content ranges from $4.42 \%$ to $24.22 \%$. Würm sediments are characterized by low $\mathrm{SiO}_{2}$ amorph and $\mathrm{C}_{\mathrm{org}}$ contents (Figure 9; Tables 10,11). Contents of $\mathrm{Fe}, \mathrm{Mn}, \mathrm{Ti}, \mathrm{K}$, and other elements vary greatly. This is mainly a function of non-uniform grain size distributions, as is also the case in Holocene sediments (Figures 6, 7, 8). Contents of those elements usually associated with clays $(\mathrm{Fe}, \mathrm{Mn}, \mathrm{Ti}, \mathrm{P}, \mathrm{K}, \mathrm{Cu}, \mathrm{Zn}$, $\mathrm{Ni}, \mathrm{Cr}$, etc.) are lower in sandy and aleuritic beds. Carbonates are represented mineralogically by calcite (7.1\%), aragonite $(10.4 \%)$, and some dolomite. One sandy interbed (Sample 2-1, 62-67 cm) is characterized by its anomalously high carbonate content $(41.48 \%$ of $\mathrm{CaCO}_{3}$ ). This interbed is characterized by the lowest (for Site 380) contents of $\mathrm{Fe}, \mathrm{Ti}$, and many other elements.

Sediments from Unit B-1-d (R-W) are represented by non- and low-calcareous clay and silty clay mud. Some of the beds are enriched in diatoms and organic matter (3.38-6.05\% $\mathrm{SiO}_{2}$ amorph; $1.08-1.94 \%$ Corg). Interbeds of terrigenous sands and silts are also present in the section, but were not studied in detail. $\mathrm{Fe}, \mathrm{Mn}$, and $\mathrm{Ti}$ contents range considerably, minimum values characterizing sands and aleurite beds as well as highcalcareous oozes. Minor elements were mainly studied in oozes enriched in diatoms and organic matter, where their contents are almost constant.
Sediments from Unit B-1-e (R) comprise terrigenous and silty clay mud with sand and aleuritic beds. These sediments differ from those of Unit B-1-d, primarily in lower and more uniformly distributed $\mathrm{SiO}_{2}$ amorph and Corg contents (Table 10). Average chemical composition is mostly similar to that of the upper-New Euxinic mud (Figure 8). Unit B-1-f (M-R) mainly consists of terrigenous low-calcareous aleurite-pelitic muds. These sediments (Cores 15-19) are relatively enriched in diatoms $\left(3.96-4.08 \% \mathrm{SiO}_{2}\right.$ amorph) and $\mathrm{C}_{\text {org }}$ is slightly increased (up to $1.06 \%$ ). Contents of elements is similar to Unit B-1-e.

Units B-1-g and B-1-h (M) comprise terrigenous lowcalcareous pelitic and aleurite-pelitic muds. A considerable part of Unit B-1-h is composed of turbidites. Unit B-1-g sediments have increasing $\mathrm{CaCO}_{3}$ contents toward the bottom (up to $24.92 \%$ ), as well as having interbeds (Sample 27-3, 1-13 cm) with sharply increased $\mathrm{Fe}$ (up to $8.24 \%$ ) and $\mathrm{Mn}$ (up to $0.89 \%$ ) contents; other elements ( $\mathrm{Rb}, \mathrm{Li}$, and partly $\mathrm{Ti}, \mathrm{Cr}, \mathrm{V})$ are also locally enriched. Mineral composition of these interbedded sediments (Trimonis et al., this volume) is mainly the same as those with low $\mathrm{Fe}$ and Mn contents, suggesting that the elements occur in the interbeds as hydroxides.

One interbed (Sample 35-3, 19-42 cm) is made of lowsiliceous, diatomaceous muds $\left(13.15 \% \quad \mathrm{SiO}_{2}\right.$ amorph; $1.64 \% \mathrm{Corg}_{\text {) }}$; contents of most elements are low. $\mathrm{Fe}$ contents have a wide range (2.77-5.38\%); other elements have narrower ranges (Figure 9).

Unit B-2 comprises terrigenous clay and silty-clay muds (non-calcareous and low-calcareous, 4.00-12.34\% $\mathrm{CaCO}_{3}$ ) with extremely low contents of $\mathrm{SiO}_{2}$ amorph and Corg. The presence of chemogenic-diagenetic calcite $(5.1-14.2 \%)$, dolomite and siderite $(0.0-7.5 \%$ and $0.0-3.6 \%$, respectively) is characteristic of this unit's lithology. Average contents of most elements are the same as in terrigenous muds of other units. An exception is $\mathrm{K}$ and, sometimes, $\mathrm{Cu}, \mathrm{V}, \mathrm{Pb}, \mathrm{Mo}$, and $\mathrm{B}$, which are sometimes enriched (Table 10).

Some sediment interbeds from Hole 380 are characterized by increased $\mathrm{S}, \mathrm{F}, \mathrm{FeO}$, and $\mathrm{MgO}$ contents (Table 12).

As a whole, the following geochemical properties are characteristic of Site 380 .

1) The Glacial epoch deposits from this hole, as well as from Site 379, mainly consist of terrigenous (non-calcareous and low-calcareous) clays poor in organic matter, whereas Interglacial deposits consist of clays and low-siliceous (diatomaceous) muds enriched in organic matter.

2) Subunits differ in their geochemical compositions. Based on average values (Table 11), we may differentiate between Units B-1-c (W) and B-1-d (R-W). In the latter, $\mathrm{Corg}_{\mathrm{o}}, \mathrm{Cr}$, and $\mathrm{F}$ contents are higher than in any other part of the section. Unit B-1-g (R) is characterized by maximum (for the entire site) $\mathrm{Fe}, \mathrm{Mn}$, $\mathrm{P}, \mathrm{Zn}, \mathrm{Ni}$, and $\mathrm{W}$ contents, where Units B-1-h and B-2 have the highest $\mathrm{SiO}_{2}$ amorph and $\mathrm{Na}$ contents and are enriched in $\mathrm{K}, \mathrm{V}, \mathrm{Pb}, \mathrm{Mo}$, and $\mathrm{B}$.

3) Minor element contents vary in the different types of sediments. In sand-aleuritic interbeds the contents of 
TABLE 3

Chemical Composition of Bottom Sediments ${ }^{\mathrm{a}}$ at Leg 42B Sites, $10^{-4} \%$

\begin{tabular}{|c|c|c|c|c|c|c|c|c|c|c|c|c|c|}
\hline $\begin{array}{l}\text { Depth } \\
\text { (m) }\end{array}$ & $\begin{array}{r}\text { Sa } \\
\text { (Inter }\end{array}$ & $\begin{array}{l}\text { mple } \\
\text { al in } \mathrm{cm} \text { ) }\end{array}$ & $\mathrm{Fe}$ & $\mathrm{La}$ & Th & $\mathrm{Cr}$ & Hf & $\mathrm{Sc}$ & Co & $\mathrm{Eu}$ & $\mathrm{Sb}$ & $\mathrm{Ce}$ & $\mathrm{Ta}$ \\
\hline \multicolumn{14}{|c|}{ Hole 379A } \\
\hline 0.46 & 1-1, & $46-48$ & 5.7 & 22 & - & 180 & 2.1 & 18 & 19 & 0.95 & 1.6 & 50 & 1.2 \\
\hline 29.65 & $4-4$ & $15-17$ & 4.6 & 26 & 8 & 150 & 3.1 & 18 & 18 & 1.2 & 0.5 & 50 & 1.0 \\
\hline 46.90 & 6-2, & $40-42$ & 4.8 & 26 & 7 & 90 & 2.8 & 22 & 20 & 1.4 & 0.7 & 53 & - \\
\hline 64.09 & 8-1, & $9-11$ & 4.7 & 29 & 8 & - & 3.1 & 19 & 18 & 1.4 & 1.3 & 60 & 2.0 \\
\hline 67.22 & 8-3, & $22-24$ & 4.7 & 23 & 8 & 100 & 5.8 & 22 & 15 & 1.4 & 0.8 & 46 & 1.3 \\
\hline 77.07 & $9-4$, & $7-9$ & 3.6 & 25 & 9 & - & 4.3 & 16 & 13 & 1.1 & - & 65 & 0.7 \\
\hline 89.11 & 10-5, & $11-13$ & 5.0 & 34 & 9 & 130 & 3.9 & 23 & 17 & 0.6 & 0.5 & 38 & 2.0 \\
\hline 95.73 & 11-3, & $23-25$ & 4.3 & 30 & 8 & - & 4.0 & 18 & 15 & 1.1 & 1.8 & 74 & 1.3 \\
\hline 119.02 & $13-6$, & $2-4$ & 6.2 & 33 & 10 & 220 & 3.8 & 26 & 29 & 1.8 & 1.4 & 72 & 1.5 \\
\hline 144.67 & $16-4$, & $17-19$ & 4.6 & 23 & .10 & 150 & 2.2 & 23 & 17 & 0.9 & 0.5 & 54 & 2.1 \\
\hline 161.83 & 18-2, & $133-135$ & 3.8 & 21 & 7 & 100 & 3.3 & 20 & 18 & 0.9 & 0.9 & 55 & 0.6 \\
\hline 209.50 & 23-2, & $150-152$ & 3.3 & 19 & 4 & 58 & 3.0 & 15 & 13 & 0.9 & 1.2 & 32 & 1.3 \\
\hline 221.82 & 24-4, & $132-135$ & 3.0 & 24 & 7 & 180 & 1.7 & 17 & 19 & 1.2 & 1.0 & 54 & 1.5 \\
\hline 221.90 & 24-4, & $140-141$ & 3.8 & 26 & 8 & 115 & 2.7 & 16 & 15 & 1.4 & 1.3 & 67 & 0.5 \\
\hline 229.70 & $25-3$ & $120-121$ & 5.5 & - & 9 & 120 & 2.7 & 20 & 20 & 0.9 & 1.9 & 60 & 0.4 \\
\hline 229.74 & 25-3, & $124-125$ & 4.4 & 20 & 8 & 100 & 2.4 & 18 & 16 & 0.7 & 1.0 & 42 & 0.3 \\
\hline 229.78 & $25-3$ & $128-129$ & 4.0 & 20 & 6 & 87 & 1.4 & 21 & 18 & 1.0 & 1.8 & 50 & 0.2 \\
\hline 235.87 & $25-7$, & $137-139$ & 5.8 & - & 9 & 100 & 3.0 & 22 & 24 & 1.1 & 1.1 & 65 & 2.4 \\
\hline 235.92 & $25-7$, & $142-144$ & 4.8 & 20 & - & 180 & 2.1 & 20 & 20 & 1.0 & 1.0 & 270 & 0.2 \\
\hline 255.74 & $28-2$, & $24-26$ & 2.8 & 14 & - & 100 & 2.8 & 12 & 18 & 0.9 & 0.5 & 43 & 1.4 \\
\hline 264.44 & 29-1, & $94-96$ & 4.0 & 19 & - & 150 & 2.8 & 16 & 18 & 0.8 & 1.1 & 46 & 2.0 \\
\hline 269.54 & 29-5, & $4-6$ & 5.5 & 25 & 10 & 50 & 3.0 & 20 & 36 & 0.9 & 2.0 & 66 & - \\
\hline 270.86 & $29-5$, & $136-138$ & 2.0 & 8 & 2 & 29 & 0.6 & 6 & 6 & 0.4 & 0.5 & 47 & - \\
\hline 316.19 & $34-4$, & $69-71$ & 3.5 & 23 & 6 & 87 & 2.0 & 17 & 10 & 1.1 & 0.3 & 56 & 1.0 \\
\hline 350.61 & $38-2$, & $11-13$ & 4.5 & 23 & 7 & 100 & 3.8 & 17 & 17 & 1.0 & 0.4 & 37 & 0.2 \\
\hline 373.90 & $40-4$ & $140-142$ & 3.4 & 20 & - & 220 & 3.0 & 20 & 19 & 1.3 & 0.5 & 50 & 0.5 \\
\hline 373.94 & $40-4$, & $144-146$ & 5.0 & 19 & - & 180 & 2.5 & 14 & 17 & 1.1 & 0.6 & 40 & - \\
\hline 436.32 & $48-2$, & $32-34$ & 3.1 & 15 & 6 & 95 & 1.7 & 17 & 13 & 0.9 & 0.5 & 37 & 1.8 \\
\hline 436.35 & $48-2$, & $35-37$ & 3.1 & 20 & 7 & 110 & 2.8 & 14 & 15 & 0.9 & 0.5 & - & - \\
\hline 436.38 & $48-2$, & $38-40$ & 3.4 & 23 & - & 450 & 8.0 & 13 & 16 & 1.3 & 1.0 & - & 1.6 \\
\hline 460.85 & $50-5$, & $135-137$ & 3.8 & 16 & - & 200 & 2.0 & 17 & 18 & 0.7 & 1.0 & 55 & 0.2 \\
\hline 476.55 & $52-3$, & $105-114$ & 3.8 & 23 & - & 200 & 2.8 & 16 & 21 & 1.2 & 0.6 & 50 & 0.9 \\
\hline 511.83 & 56-1, & $133-143$ & 4.9 & 24 & 8 & 120 & 2.6 & 20 & 15 & 1.0 & 0.7 & 67 & 0.5 \\
\hline 532.14 & $58-2$, & $114-118$ & 4.2 & 22 & - & 180 & 2.7 & 15 & 20 & 1.3 & 1.8 & 34 & - \\
\hline 532.18 & $58-2$, & $118-122$ & 4.2 & - & 9 & 140 & 3.3 & 19 & 11 & 1.1 & 0.2 & 60 & 1.8 \\
\hline 551.35 & $60-2$ & $135-145$ & 4.5 & 24 & 10 & 140 & 2.7 & 20 & 19 & 1.4 & - & 58 & 1.3 \\
\hline 595.20 & 65-6, & $20-22$ & 3.5 & 18 & 7 & 220 & 2.7 & 17 & 14 & 0.8 & 0.5 & 58 & 0.3 \\
\hline 597.98 & $66-2$, & $48-58$ & 4.5 & 21 & 6 & 150 & 2.3 & 20 & 16 & 1.0 & 1.3 & 52 & 0.8 \\
\hline 618.66 & 68-3, & $66-68$ & 4.0 & 21 & - & 180 & 2.5 & 18 & 23 & 1.0 & 0.5 & 51 & 0.8 \\
\hline 621.73 & $68-5$, & $73-75$ & 3.5 & 17 & - & 220 & 2.2 & 15 & 19 & 1.0 & 0.5 & 25 & 0.5 \\
\hline
\end{tabular}

Hole 380

\begin{tabular}{rrrrrrrrrrrrrr}
1.87 & $1-1$, & $137-139$ & 3.6 & 28 & 5 & 70 & 5.3 & 14 & 12 & 1.2 & 1.2 & - & 1.2 \\
2.88 & $1-2$, & $138-140$ & 4.5 & 12 & 8 & 100 & 3.8 & 20 & 18 & 1.4 & 1.3 & 70 & 2.0 \\
10.12 & $2-1$, & $62-67$ & 0.8 & 8 & 1.5 & - & 1.0 & 3 & 3 & 0.3 & 0.7 & 24 & 0.6 \\
43.43 & $5-4$, & $93-95$ & 3.0 & 27 & - & 100 & 4.7 & 13 & 14 & 1.0 & 0.7 & 50 & 1.6 \\
67.83 & $8-1$, & $133-135$ & 4.6 & 34 & 6 & 90 & 3.7 & 20 & 19 & 1.4 & 0.2 & 60 & 2.0 \\
76.87 & $9-1$, & $87-88$ & 4.5 & 32 & 2 & 90 & 3.1 & 23 & 18 & 1.3 & 0.9 & 60 & 2.5 \\
105.80 & $12-1$, & $130-131$ & 4.6 & - & 7 & 90 & 4.1 & 19 & 17 & 1.2 & 0.7 & 50 & 1.5 \\
157.81 & $17-4$, & $131-133$ & 3.6 & 47 & - & - & 21.7 & 16 & 13 & 1.6 & 0.2 & - & 2.5 \\
166.18 & $18-4$, & $18-20$ & 5.8 & 41 & 9 & 100 & 4.0 & 24 & 24 & 1.5 & 2.3 & 60 & 1.8 \\
171.17 & $19-1$, & $17-19$ & 4.2 & 28 & 12 & 100 & 5.0 & 18 & 15 & 1.2 & 0.4 & 50 & 1.3 \\
211.93 & $23-2$, & $143-145$ & 4.0 & 32 & 11 & 80 & 3.8 & 18 & 15 & 1.3 & 0.8 & 76 & 1.5 \\
251.38 & $27-3$, & $138-140$ & 2.7 & 32 & 2.5 & 17 & 0.8 & 7 & 35 & 0.5 & 1.5 & - & 0.2 \\
300.51 & $32-5$, & $1-10$ & 4.6 & 28 & 10 & 90 & 3.7 & 20 & 18 & 1.3 & 1.6 & - & 2.0 \\
336.56 & $36-3$, & $106-118$ & 4.0 & - & 12 & - & 3.0 & 14 & 18 & 1.4 & - & 53 & 0.8 \\
353.30 & $38-2$, & $30-31$ & 17.0 & 24 & 7 & 50 & - & 15 & 12 & 0.9 & 1.7 & 67 & 0.2 \\
363.03 & $39-2$, & $53-54$ & 5.0 & 31 & 6 & 90 & 4.0 & 22 & 20 & 1.3 & 1.8 & 50 & 1.8 \\
371.26 & $40-1$, & $76-78$ & 18.0 & 29 & 5.5 & 80 & 3.5 & 15 & 11 & 1.3 & 0.5 & 70 & - \\
Hole 380A & & & & & & & & & & & & \\
335.95 & $1-3$, & $45-60$ & 4.6 & 44 & 9 & - & 3.5 & 9 & 40 & 1.9 & - & - & 1.3 \\
353.74 & $3-2$, & $74-87$ & 3.8 & - & 7 & - & 3.7 & 12 & 14 & 1.2 & 3.2 & 64 & - \\
363.63 & $4-2$, & $113-128$ & 4.7 & 29 & 9 & - & 2.5 & 16 & 13 & 1.2 & - & 59 & 1.2 \\
\hline
\end{tabular}


TABLE 3 - Continued

\begin{tabular}{|c|c|c|c|c|c|c|c|c|c|c|c|c|c|}
\hline $\begin{array}{l}\text { Depth } \\
\text { (m) }\end{array}$ & $\begin{array}{r}\mathrm{S} \\
\text { (Inter }\end{array}$ & $\begin{array}{l}\text { mple } \\
\text { al in } \mathrm{cm} \text { ) }\end{array}$ & $\mathrm{Fe}$ & $\mathrm{La}$ & Th & $\mathrm{Cr}$ & Hf & $\mathrm{Sc}$ & Co & $\mathrm{Eu}$ & $\mathrm{Sb}$ & $\mathrm{Ce}$ & $\mathrm{Ta}$ \\
\hline \multicolumn{14}{|c|}{ Hole $380 \mathrm{~A}$ - Continued } \\
\hline 402.61 & 8-3, & $61-63$ & - & 20 & - & - & - & - & - & - & - & - & - \\
\hline 420.12 & $10-2$ & $62-74$ & 4.1 & 23 & 10 & - & 3.0 & 17 & 14 & 1.3 & 1.2 & - & 1.3 \\
\hline 447.50 & 13-1, & $100-111$ & 4.1 & 18 & 8 & - & 2.0 & 15 & 12 & 1.1 & - & 68 & - \\
\hline 467.40 & $15-2$ & $40-42$ & 2.9 & 20 & 1.4 & - & 1.2 & 10 & 8 & 0,9 & - & - & - \\
\hline 481.56 & $17-2$ & $56-70$ & - & - & 5 & - & 1.8 & - & - & - & - & - & - \\
\hline 501.81 & 19-3, & $31-33$ & - & 22 & 12 & - & 2.1 & 8 & 6 & 0.7 & 0.5 & 50 & 0.5 \\
\hline 535.84 & $22-3$ & $84-96$ & - & 21 & 7 & - & 1.3 & 10 & 10 & 0.8 & 1.8 & - & 0.6 \\
\hline 562.45 & $25-2$ & $45-61$ & 4.6 & 16 & 1.4 & - & 1.1 & 10 & 12 & 0.6 & 2.5 & - & - \\
\hline 563.26 & $25-2$ & $126-136$ & 1.8 & 18 & 8 & - & 2.5 & 14 & - & 1.2 & 3.7 & 133 & - \\
\hline 588.34 & $27-6$ & $134-137$ & 2.9 & 9 & 1.0 & - & 0.1 & - & 4 & 0.4 & - & - & 0.3 \\
\hline 617.56 & $32-1$, & $6-9$ & - & 26 & 11 & - & 2.0 & 15 & 15 & 1.3 & 2.1 & 60 & 0.7 \\
\hline 639.77 & $34-3$ & $27-44$ & 3.2 & 26 & 8 & - & 1.2 & 13 & 12 & 1.1 & 1.0 & - & 0.8 \\
\hline 657.30 & $36-2$ & $30-42$ & 3.9 & 13 & 8 & - & 0.5 & 17 & 12 & 1.3 & 1.9 & 12 & 2.9 \\
\hline 672.64 & $37-6$, & $14-19$ & 4.8 & 31 & 13 & - & - & 21 & 21 & 1.6 & 2.9 & 56 & 0.6 \\
\hline 700.15 & $40-5$, & $65-67$ & 1.7 & 20 & 3 & - & 1.4 & 7 & 7 & 0.8 & 1.3 & 51 & - \\
\hline 703.53 & $41-1$ & $53-55$ & 26.3 & 3 & - & - & - & 12 & 7 & - & - & - & - \\
\hline 717.33 & $42-4$ & $33-35$ & 16.3 & - & 1.5 & - & 1.4 & 9 & 10 & 2.9 & - & - & 0.9 \\
\hline 752.55 & $46-7$ & $55-57$ & 3.4 & 12 & 6 & - & 1.9 & 12 & 9 & 1.1 & 2.1 & - & 0.4 \\
\hline 784.50 & $49-5$ & $0-15$ & - & 15 & 4 & - & 1.0 & 7 & - & 0.7 & 1.5 & - & - \\
\hline 795.33 & $50-5$ & $133-135$ & 4.4 & 26 & 8 & - & 3.2 & 19 & 17 & 1.0 & 0.5 & 47 & 0.5 \\
\hline 800.56 & $51-3$ & $6-8$ & - & - & 9 & - & 3.3 & 10 & 8 & 0.8 & 0.5 & 10 & 0.4 \\
\hline 816.21 & $52-6$ & $121-123$ & 2.6 & - & 5 & - & 1.9 & 10 & 9 & 1.1 & 2.1 & - & 0.4 \\
\hline 816.27 & $52-6$ & $127-129$ & 2.5 & 17 & 5 & - & 1.6 & 10 & 9 & 0.8 & - & - & 0.4 \\
\hline 816.33 & $52-6$ & $133-134$ & 2.8 & 34 & 10 & - & 2.1 & 14 & 19 & 1.7 & 4.3 & - & 0.5 \\
\hline 816.36 & $52-6$ & $136-138$ & 2.0 & 20 & 4 & - & - & 8 & 8 & 0.8 & - & - & - \\
\hline 840.71 & $55-4$ & $21-32$ & 3.6 & 18 & 3 & - & - & 15 & 13 & 1.0 & 2.3 & - & - \\
\hline 849.08 & $56-3$ & $58-60$ & 3.4 & 26 & 4 & - & 1.8 & 10 & 20 & 0.8 & 3.0 & 45 & - \\
\hline 921.74 & 64-1, & $24-26$ & 2.0 & 4 & 3 & - & 0.4 & 2 & 2 & 0.1 & - & - & - \\
\hline 933.92 & $65-2$ & $142-144$ & 2.2 & 10 & 3 & - & 14.7 & 6 & 4 & 0.5 & 0.8 & - & 0.3 \\
\hline 940.72 & $66-1$ & $22-24$ & 2.3 & 9 & 4 & - & 1.1 & 8 & 11 & 1.0 & - & - & - \\
\hline 980.63 & $71-2$ & $13-21$ & 3.9 & 22 & 3 & - & 3.0 & 16 & 20 & 1.3 & - & 58 & - \\
\hline 1055.96 & $79-2$ & $46-48$ & 4.5 & 13 & 7 & - & 0.5 & 14 & 14 & 1.0 & - & 66 & 0.4 \\
\hline \multicolumn{14}{|l|}{ Site 381} \\
\hline 0.68 & $1-1$ & $68-70$ & 2.9 & 16 & 7 & - & 2.3 & 10 & 12 & 0.8 & 0.9 & - & 0.4 \\
\hline 49.85 & $6-2$ & $85-100$ & - & 24 & - & - & - & - & - & - & - & - & - \\
\hline 61.25 & $7-3$ & $125-150$ & 6.3 & - & 17 & - & 5.5 & 26 & 22 & 2.2 & 0.5 & 144 & 1.3 \\
\hline 86.75 & 10-1, & $125-150$ & 4.8 & 29 & 15 & - & 3.9 & 19 & 15 & 1.6 & 0.5 & 88 & 0.8 \\
\hline 106.15 & $17-2$ & $15-16$ & 2.6 & 13 & - & - & 0.5 & 8 & 4 & 0.6 & 0.5 & - & 0.5 \\
\hline 117.90 & 13-3, & $90-105$ & - & 18 & 10 & - & 2.1 & - & - & 1.2 & 0.1 & 56 & 0.7 \\
\hline 150.00 & $16-6$ & $0-20$ & 3.8 & 19 & 9 & - & 1.9 & 16 & 14 & 1.1 & 0.5 & 51 & 0.7 \\
\hline 171.00 & 19-1, & $0-15$ & 6.6 & 10 & 4 & - & 1.1 & 10 & 6 & 0.3 & 0.5 & - & - \\
\hline 174.00 & 19-3, & $40-42$ & 4.4 & 26 & 12 & - & 3.2 & 20 & 19 & 1.3 & 0.5 & 53 & 0.4 \\
\hline 198.40 & $22-3$ & $40-50$ & - & 22 & 14 & - & 2.8 & 22 & - & 1.5 & 0.2 & 66 & - \\
\hline 209.48 & 24-1, & $48-63$ & 5.3 & 16 & 9 & - & 2.1 & - & 12 & 1.1 & 0.5 & 62 & 0.2 \\
\hline 239.50 & $27-2$ & $50-65$ & 3.9 & 21 & 12 & - & 2.2 & 14 & 19 & 1.6 & 0.12 & 75 & 0.8 \\
\hline 249.28 & $28-2$ & $78-80$ & 3.9 & 22 & 9 & - & 2.2 & - & 16 & 1.3 & 1.5 & 54 & 0.9 \\
\hline 258.00 & $29-2$ & $0-15$ & - & 22 & - & - & - & - & - & - & - & 45 & - \\
\hline 278.82 & $31-3$ & $32-34$ & 4.1 & 26 & 11 & - & 3.4 & 18 & 17 & 1.7 & 1.7 & 53 & 0.3 \\
\hline 286.75 & $32-2$ & $25-27$ & 2.0 & 14 & 5 & - & 0.8 & 7 & 6 & 0.7 & 1.2 & - & - \\
\hline 296.86 & $33-2$ & $86-88$ & 2.1 & 14 & 5 & - & - & 6 & 8 & 0.8 & 1.7 & - & 0.3 \\
\hline 310.23 & $34-5$ & $23-33$ & 0.7 & 6 & 16 & - & - & 2 & 3 & 0.4 & 0.6 & 27 & 0.1 \\
\hline 314.60 & $35-1$, & $110-120$ & - & 37 & 12 & - & - & 19 & - & 1.3 & 0.2 & 68 & - \\
\hline 324.94 & $36-2$ & $44-46$ & 4.3 & 21 & 13 & - & 2.6 & 17 & 26 & - & 3.5 & 75 & 0.5 \\
\hline 333.40 & $37-1$, & $90-92$ & 2.7 & 16 & 7 & - & 2.6 & 10 & 6 & - & - & 34 & 0.6 \\
\hline 333.84 & $37-1$ & $134-136$ & 1.7 & 11 & 5 & _ & - & 8 & 8 & 0.4 & 0.9 & 21 & 0.2 \\
\hline 333.96 & $37-1$ & $146-148$ & - & 10 & - & - & 2.7 & 6 & - & 0.6 & - & - & 0.4 \\
\hline 381.40 & $42-1$, & $140-145$ & - & 18 & - & - & 3.6 & 16 & - & 1.3 & - & - & - \\
\hline 441.38 & $48-4$ & $88-90$ & 3.4 & 28 & 16 & - & 9.7 & 13 & 12 & 1.5 & - & - & 0.8 \\
\hline 448.70 & $49-2$ & $70-83$ & 5.8 & 28 & 13 & - & - & 23 & 20 & 1.6 & 0.5 & 50 & 1.4 \\
\hline 476.00 & $52-1$ & $100-110$ & 4.7 & - & 14 & - & 3.4 & 19 & 30 & 1.8 & 0.5 & 76 & 1.2 \\
\hline 477.15 & $52-2$ & $65-67$ & 4.3 & 20 & 13 & - & 4.1 & 19 & 21 & 1.1 & 0.1 & 62 & 0.8 \\
\hline 486.54 & $53-2$ & $54-55$ & 3.0 & 9 & 12 & - & 4.3 & 11 & 105 & 1.2 & 0.5 & 48 & 0.4 \\
\hline 502.73 & $54-6$ & $123-125$ & 16.3 & 19 & 11 & - & 4.6 & 17 & 21 & 1.3 & 1.4 & 50 & 0.8 \\
\hline
\end{tabular}

aStudied by instrumental and neutron-activation analysis and determined by INAA. 
TABLE 4

Average Contents of Components and Elements in Hole 379A Units

\begin{tabular}{|c|c|c|c|c|c|c|c|c|c|c|c|c|c|c|c|c|c|c|c|c|c|c|}
\hline \multirow[b]{2}{*}{ Unit } & \multirow[b]{2}{*}{ Epoch } & \multirow[b]{2}{*}{$\mathrm{CaCO}_{3}$} & \multicolumn{5}{|c|}{ Concentration (\%) } & \multicolumn{15}{|c|}{ Concentration $\left(10^{-4} \%\right)$} \\
\hline & & & $\mathrm{C}_{\text {org }}$ & $\mathrm{Fe}$ & $\mathrm{Mn}$ & $\mathrm{Ti}$ & K & $\mathrm{Na}$ & $\mathrm{Cu}$ & $\mathrm{Zn}$ & $\mathrm{Ni}$ & Co & $\mathrm{Cr}$ & $\mathrm{v}$ & $\mathrm{Cd}$ & $\mathrm{Li}$ & $\mathrm{Rb}$ & Cs & $\mathrm{Pb}$ & $\mathrm{Sn}$ & F & B \\
\hline \multirow[t]{2}{*}{3} & w & a 16.91 & 0.68 & 4.48 & 0.09 & 0.45 & 1.64 & 1.07 & 50 & 87 & 148 & 22 & 164 & 100 & 6 & 18 & 44 & 4.3 & 18 & 1.7 & 1047 & 89 \\
\hline & & - & 0.82 & 5.48 & 0.11 & 0.55 & 2.00 & 1.30 & 61 & 106 & 180 & 27 & 199 & 121 & 7 & 22 & 53 & 5.2 & 22 & 2.1 & 1280 & 108 \\
\hline $4-5$ & & - & 1.13 & 4.89 & 0.12 & 0.49 & 2.01 & 1.51 & 64 & 104 & 134 & 43 & 135 & 147 & 8 & 24 & 58 & 4.9 & 23 & 1.9 & 411 & 115 \\
\hline \multirow[t]{2}{*}{6} & $\mathbf{R}$ & a $\quad 13.92$ & 0.61 & 4.47 & 0.08 & 0.44 & 1.79 & 0.88 & 51 & 92 & 156 & 41 & 150 & 165 & 6 & 24 & 59 & 4.9 & 19 & 1.6 & 343 & 154 \\
\hline & & - & 0.71 & 5.27 & 0.09 & 0.51 & 2.09 & 1.04 & 60 & 108 & 183 & 48 & 177 & 194 & 7 & 28 & 70 & 5.8 & 22 & 1.9 & 401 & 180 \\
\hline 7 & M-R & 19.10 & 1.59 & 4.42 & 0.09 & 0.43 & 1.73 & 0.76 & 57 & 93 & 136 & 42 & 140 & 147 & 6 & 21 & 58 & 4.5 & 15 & 1.5 & 582 & 101 \\
\hline 8 & & - & 0.56 & 5.69 & 0.10 & 0.55 & 2.23 & 1.03 & 55 & 104 & 178 & 39 & 200 & 198 & 7 & 29 & 70 & 6.0 & 16 & 1.6 & 874 & 177 \\
\hline \multirow[t]{2}{*}{9} & G-M & a $\quad 24.40$ & 0.53 & 4.01 & 0.08 & 0.38 & 1.47 & 0.83 & 48 & 82 & 125 & 28 & 160 & 150 & 6 & 20 & 52 & 4.9 & 11 & 1.1 & 518 & 102 \\
\hline & & - & 0.70 & 5.38 & 0.11 & 0.51 & 1.97 & 1.11 & 64 & 110 & 168 & 38 & 215 & 201 & 8 & 27 & 70 & 6.6 & 15 & 1.5 & 762 & 136 \\
\hline & - & 0.54 & 4.4 & 0.0 & 0.4 & 1.7 & 0.9 & 48 & 88 & 14 & 32 & 159 & 14 & 6 & 23 & 56 & 4 & 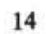 & 1. & 541 & 116 \\
\hline \multicolumn{2}{|c|}{ Diat. S. } & - & 1.60 & 4.48 & 0.10 & 0.44 & 1.76 & 1.13 & 57 & 94 & 130 & 44 & 132 & 138 & 7 & 23 & 59 & 4.8 & 20 & 1.6 & 482 & 100 \\
\hline & - & 0.55 & 3.32 & 0.09 & 0.29 & 1.23 & 0.62 & 43 & 70 & 92 & 47 & 119 & 130 & 6 & 15 & 41 & 2.8 & - & 1.1 & 390 & - \\
\hline
\end{tabular}

Note: Terr. S. = terrigenous sediments with $\mathrm{CaCO}_{3}$ content $<30 \%$ (Units $3,6,8,9$ ); Diat. S. $=$ diatom sediments (Units 4 and 7 ); Carb. S. = carbonate sediments with $\mathrm{CaCO}_{3}$ content $>30 \%$ (all holes); $\mathrm{a}=$ average in natural sediments; $\mathrm{b}=$ average on a carbonate and organic matter $\left(\mathrm{C}_{\mathrm{org}} \times 2\right)$ free basis.

most minor elements are decreased. In low-manganous and low-ferruginous oozes, the amounts of $\mathrm{Rb}$ and $\mathrm{Ti}$ and, sometimes, $\mathrm{Cr}$ and $\mathrm{V}$ are increased. In lowsiliceous diatomaceous oozes, most element contents are decreased relative to their contents in clays.

4) The ratios of average contents of the elements studied to their clarkes (Ko) are peculiar for each of the elements as found in sedimentary rocks (Tables 9,11 ). The contents of $\mathrm{Fe}, \mathrm{Mn}$, and $\mathrm{Na}$ throughout the whole section are higher than their Ko, whereas, in the Interglacial deposits, Se and Mo contents are higher because these elements are associated with the presence of organic matter. The contents of $\mathrm{Cu}, \mathrm{Ni}, \mathrm{Co}, \mathrm{Li}, \mathrm{Rb}$, $\mathrm{Cs}$, and $\mathrm{Zn}$ in the section are lower.

This reflects a close genetic connection between sediments from Hole 380 and the products of weathering of crystalline rock masses, represented by old metamorphic series of acid-intermediate composition, and by younger acidic intrusions. These rock masses occur in the vicinity of Site 380 (at the Bulgaria-Turkey border) as well as in the northwestern area drained by the largest Black Sea rivers: the Dneiper, the Dneister, and the Danube.

6) In contrast to Site 379 , Hole 380 deposits are significantly rich in $\mathrm{Li}, \mathrm{Rb}, \mathrm{Zn}$ and poor in $\mathrm{Cu}, \mathrm{Ni}, \mathrm{Co}$, $\mathrm{Cr}$, and $\mathrm{Se}$. This mainly results from a large percentage of weathered acid and intermediate rock products in Hole 380 and of basic-intermediate rocks at Site 379.

\section{Hole 380A}

Hole $380 \mathrm{~A}$ sediments are divided into eight units. Two are Eopleistocene in age; the others are Pliocene and Miocene.

Unit B-2 consists of terrigenous (non-calcareous and low-calcareous) muds, often rather finely dispersed (over $90 \%$ comprises the $<0.01 \mathrm{~mm}$ fraction) and with low contents of organic matter (usually $0.27-0.60 \%$ Corg). Corg amounts to $1.32 \%$ in a single interbed (3-2, $74-87 \mathrm{~cm})$. High-calcareous ooze beds poor in organic matter $\left(55.34 \% \mathrm{CaCO}_{3}, 0.50 \% \mathrm{Corg}\right)$ are rare $(9-6,97-$ $106 \mathrm{~cm}$ ) (Tables 13, 14).

As shown by Trimonis et al. (this volume), clay matter consists basically of illite. Content of montmorillonite and chlorite is high in individual beds; kaolinite is rare.

According to X-ray analyses, calcite is the basic carbonate, with dolomite and siderite being common. Dolomite makes up the total carbonate fraction only in localized beds.

The content of amorphous silica is low $(2.50 \%)$ at most studied levels. Relatively increased contents were observed only in an interbed enriched in organic matter (Figures 11, 12). Interbeds of low-ferruginous muds $(5.15-5.56 \% \mathrm{Fe})$ with somewhat increased $\mathrm{Ti}$ contents $(0.50-0.55 \%)$ occur in the middle of the unit (Cores 810).

Quantitative variations of most microelements are insignificant. Relatively high $\mathrm{Cr}$ and $\mathrm{B}$ contents are present in a bed of sapropel. Decreased contents of most micro-elements are characteristic of highcalcareous oozes. Higher $\mathrm{Cr}$ and B contents occur in some interbeds of clays.

Unit B-3, also Eopleistocene in age, consists mainly of terrigenous pelitic and aleurite-pelitic muds with relatively constant carbonate content $\left(63.5 \% \mathrm{CaCO}_{3}\right)$. Thin rhythms $(2-8 \mathrm{~cm})$ consisting of non-calcareous, low- and high-calcareous oozes occur as laminated varve-like deposits. Clay material in these sediments is basically represented by illite and montmorillonite. Chlorite content is markedly lower, as a rule, and kaolinite is not common. Calcite is mostly the dominant carbonate, with dolomite and siderite as sporadic admixtures.

In most cases the oozes are characterized by low contents of organic matter $(0.29-0.60 \%$, sometimes $0.68-0.82 \%$ Corg). Corg exceeds $1.0 \%$ only in individual beds of clay (30-1, 108-120 cm, etc.). The low content of amorphous silica (mostly up to $2.35 \%$ ) in this unit indicates a small admixture of diatoms. A higher amount of $\mathrm{SiO}_{2}$ amorph (7.49\%) was found in a single interbed of non-calcareous clay $(17-2,111-113 \mathrm{~cm})$. Microfaunal and floral elements were not identified in our study, but analysis of smear slides on board ship demonstrated an increase in diatom concentration in this core.

The $\mathrm{Fe}, \mathrm{Mn}$, and $\mathrm{Ti}$ contents show frequent and marked variations which are closely correlated 
TABLE 5

Average Concentrations of $\left(10^{-4} \%\right)$ Elements in Black Sea Holes (the data of INAA)

\begin{tabular}{|c|c|c|c|c|c|c|c|c|c|c|}
\hline Unit & \multicolumn{2}{|l|}{ Epoch } & $\mathrm{La}$ & Th & $\mathrm{Hf}$ & Sc & $\mathrm{Eu}$ & $\mathrm{Sb}$ & $\mathrm{Ce}$ & $\mathrm{Ta}$ \\
\hline \multicolumn{11}{|c|}{ Hole 379} \\
\hline 3 & w & $\begin{array}{l}\mathrm{a} \\
\mathrm{b}\end{array}$ & $25(3)$ & $\begin{array}{l}7.5 \\
9.5\end{array}$ & $\begin{array}{l}2.7 \\
3.4\end{array}$ & ${ }_{25}^{19}(3)$ & $\begin{array}{l}1.2(3) \\
1.5\end{array}$ & $\begin{array}{l}0.9(3) \\
1.3\end{array}$ & $\begin{array}{l}51 \\
67\end{array}$ & $\begin{array}{l}1.1 \\
1.5\end{array}$ \\
\hline $4-5$ & R-W & a & $28(5)$ & $\begin{array}{r}8.4 \\
10.0\end{array}$ & $\begin{array}{l}4.2 \\
4.9\end{array}$ & $20(5)$ & $\begin{array}{l}1.1 \\
1.3\end{array}$ & $1.1(4)$ & $\begin{array}{l}57 \\
66\end{array}$ & $\begin{array}{l}1.5 \\
1.7\end{array}$ \\
\hline 6 & $\mathrm{R}$ & $\mathrm{a}$ & $\begin{array}{l}24(4) \\
28\end{array}$ & $\begin{array}{l}7.8 \\
9.0\end{array}$ & $3.1(4)$ & $25(4)$ & $\begin{array}{l}1.1 \\
1.3\end{array}$ & $\begin{array}{l}1.0 \\
1.1\end{array}$ & $\begin{array}{l}53 \\
63\end{array}(4)$ & $\begin{array}{l}1.4 \\
1.6\end{array}$ \\
\hline 7 & $M-R$ & $\mathrm{a}$ & $26(11)$ & $9.4(8)$ & $2.3(11)$ & ${ }_{22}^{17}(11)$ & $0.9(11)$ & $\begin{array}{l}1.2 \\
1.5\end{array}$ & $\begin{array}{l}74 \\
94\end{array}$ & $\begin{array}{l}1.0(9) \\
1.3\end{array}$ \\
\hline 8 & M & a & $26(7)$ & $\begin{array}{l}6.5 \\
8.0\end{array}$ & $\begin{array}{l}3.4(7) \\
4.2\end{array}$ & $16(7)$ & $\begin{array}{l}1.1 \\
1.3\end{array}(7)$ & $\begin{array}{l}0.5 \\
0.7\end{array}$ & $\begin{array}{l}44 \\
56\end{array}$ & $\begin{array}{l}1.0 \\
1.3\end{array}$ \\
\hline 9 & G-M & $\begin{array}{l}\mathrm{a} \\
\mathrm{b}\end{array}$ & $\begin{array}{l}21 \\
26\end{array}(9)$ & $\begin{aligned} & 8.0(5) \\
& 10.2\end{aligned}$ & $\begin{array}{l}2.6 \\
3.2\end{array}$ & $\frac{18}{22}(10)$ & $\begin{array}{l}1.0(10) \\
1.3\end{array}$ & $\begin{array}{l}0.8(9) \\
1.0\end{array}$ & $\begin{array}{l}51 \\
64\end{array}(10)$ & $\begin{array}{l}0.8 \\
1.0\end{array}$ \\
\hline \multicolumn{3}{|c|}{ Average for hole a } & $\begin{array}{l}22 \\
27\end{array}$ & $\begin{array}{l}7.6 \\
9.4\end{array}$ & $\begin{array}{l}2.9 \\
3.6^{(40)}\end{array}$ & $\begin{array}{l}18(40) \\
22\end{array}$ & $\begin{array}{l}1.0(40) \\
1.3\end{array}$ & $\begin{array}{l}0.9 \\
1.2^{(38)}\end{array}$ & $\begin{array}{l}58 \\
72\end{array}(38)$ & $\begin{array}{l}1.1(34) \\
1.3^{(34)}\end{array}$ \\
\hline \multicolumn{11}{|c|}{ Hole 380} \\
\hline B-1-c & w & a & ${ }_{23}^{19}(4)$ & $\begin{array}{l}4.6 \\
6.2^{(3)}\end{array}$ & $\begin{array}{l}3.7 \\
4.6\end{array}(4)$ & $\begin{array}{l}12 \\
15\end{array}(4)$ & $\begin{array}{l}1.0(4) \\
1.2^{(4)}\end{array}$ & $\begin{array}{l}1.0(4) \\
1.3\end{array}$ & ${ }_{61}^{48}(3)$ & $\begin{array}{l}1.4(4) \\
1.7\end{array}$ \\
\hline$B-1-d$ & R-W & $\begin{array}{l}\mathrm{a} \\
\mathrm{b}\end{array}$ & $\begin{array}{l}34(1) \\
38\end{array}$ & $7_{7}^{6}(1)$ & $\begin{array}{l}3.7 \\
4.2\end{array}$ & $23(1)$ & $\begin{array}{l}1.4(1) \\
1.6\end{array}$ & $\begin{array}{l}0.2(1) \\
0.2\end{array}$ & $\begin{array}{l}60 \\
69\end{array}$ & $\begin{array}{l}2.0(1) \\
2.3\end{array}$ \\
\hline B-1-e & R & $\begin{array}{l}\mathrm{a} \\
\mathrm{b}\end{array}$ & $\begin{array}{l}32 \\
34\end{array}$ & $\begin{array}{l}4.5(2) \\
5.0\end{array}$ & $\begin{array}{l}3.6(2) \\
4.0\end{array}$ & $\begin{array}{l}21 \\
23\end{array}$ & $\begin{array}{l}1.2 \\
1.3\end{array}$ & $\begin{array}{l}0.8(2) \\
0.9^{(2)}\end{array}$ & ${ }_{61}^{55}(2)$ & $\begin{array}{l}2.0 \\
2.2\end{array}$ \\
\hline B-1-f & M-R & $\begin{array}{l}\mathrm{a} \\
\mathrm{b}\end{array}$ & $\begin{array}{l}39 \\
45\end{array}(3)$ & $\begin{array}{l}10.5(2) \\
12.1\end{array}$ & $\begin{array}{l}10.2(3) \\
11.8\end{array}$ & ${ }_{22}^{19}(3)$ & $\begin{array}{l}1.4(3) \\
1.6\end{array}$ & $\begin{array}{l}1.0 \\
1.2\end{array}$ & $\begin{array}{l}55 \\
63\end{array}(3)$ & $\begin{array}{l}1.9(3) \\
2.2\end{array}$ \\
\hline B-1-g & $\mathrm{M}$ & a & $\begin{array}{l}32 \\
36\end{array}$ & $\begin{array}{l}6.8 \\
7.6\end{array}$ & $\begin{array}{l}2.3 \\
2.6\end{array}$ & $12(2)$ & $\begin{array}{l}0.9 \\
1.0\end{array}$ & $\begin{array}{l}1.2 \\
1.3\end{array}$ & $85(1)$ & $\begin{array}{l}0.8 \\
0.9\end{array}$ \\
\hline B-1-h & M & a & $\begin{array}{l}28 \\
34\end{array}$ & $\begin{array}{l}10.0 \\
12.0\end{array}$ & $\begin{array}{l}3.7 \\
4.5\end{array}$ & $\begin{array}{l}20.0 \\
24.0\end{array}$ & $\begin{array}{l}1.3 \\
1.6\end{array}$ & $\begin{array}{l}1.6 \\
2.0\end{array}$ & - & $\begin{array}{l}2.0 \\
2.4\end{array}$ \\
\hline B-2 & Eopl. & a & $\begin{array}{l}28 \\
31\end{array}$ & $\begin{array}{l}7.7 \\
8.5\end{array}$ & $\begin{array}{l}3.5 \\
3.8\end{array}$ & $\begin{array}{l}16.0^{(4)} \\
18.0^{(4)}\end{array}$ & $\begin{array}{l}1.2(4) \\
1.4\end{array}$ & $\begin{array}{l}1.3(3) \\
1.4\end{array}$ & $\begin{array}{l}60(4) \\
66\end{array}$ & $\begin{array}{l}0.9(3) \\
1.0\end{array}$ \\
\hline Avera & for hole & a & ${ }_{33}^{29}(15)$ & $\begin{array}{l}6.9(15) \\
8.0^{(15)}\end{array}$ & $\begin{array}{l}4.7 \\
5.4\end{array}$ & $\begin{array}{l}16.0 \\
19.0\end{array}$ & $\begin{array}{l}1.2(17) \\
1.4\end{array}$ & $\begin{array}{l}1.0 \\
1.2\end{array}$ & $\begin{array}{l}57 \\
65\end{array}$ & $\begin{array}{l}1.5(16) \\
1.7\end{array}$ \\
\hline \multicolumn{11}{|c|}{ Hole $380 \mathrm{~A}$} \\
\hline B-2 & Eopl. & $\begin{array}{l}\mathrm{a} \\
\mathrm{b}\end{array}$ & $\begin{array}{l}29 \\
32\end{array}$ & $\begin{array}{r}9.5 \\
10.8\end{array}$ & $\begin{array}{l}3.2 \\
3.6\end{array}$ & $\begin{array}{l}13 \\
15\end{array}(4)$ & $\begin{array}{l}1.4(4) \\
1.6\end{array}$ & $\begin{array}{l}2.2 \\
2.6\end{array}$ & $\begin{array}{l}61 \\
72\end{array}$ & $\begin{array}{l}1.3(3) \\
1.4\end{array}$ \\
\hline B-3 & Eopl. & $\begin{array}{l}\mathrm{a} \\
\mathrm{b}\end{array}$ & $\begin{array}{l}20 \\
29\end{array}$ & $\begin{array}{l}6.2(10) \\
9.5\end{array}$ & $\begin{array}{l}1.5(10) \\
2.3\end{array}$ & $\begin{array}{l}11 \\
16\end{array}(9)$ & $\begin{array}{l}0.9(9) \\
1.3\end{array}$ & $\begin{array}{l}1.9(4) \\
2.9^{(4)}\end{array}$ & $\begin{array}{l}78 \\
95\end{array}$ & $\begin{array}{l}0.6_{(5)} \\
1.0\end{array}$ \\
\hline B-4a & $\mathrm{N}_{2}$ & $\begin{array}{l}\mathrm{a} \\
\mathrm{b}\end{array}$ & $\begin{array}{l}17(4) \\
17\end{array}$ & $\begin{array}{l}6.4(4) \\
6.8\end{array}$ & $\begin{array}{l}1.1(3) \\
1.5\end{array}$ & $\begin{array}{l}13 \\
15\end{array}(5)$ & $\begin{array}{l}1.6(4) \\
2.4\end{array}$ & $\begin{array}{l}2.0 \\
2.0\end{array}$ & $\begin{array}{l}40 \\
40\end{array}$ & $\begin{array}{l}1.5(3) \\
1.8\end{array}$ \\
\hline B-4-b & $\mathrm{N}_{2}$ & $\begin{array}{l}\mathrm{a} \\
\mathrm{b}\end{array}$ & $\begin{array}{l}21 \\
39\end{array}$ & $\begin{array}{r}6.4(8) \\
11.8\end{array}$ & $\begin{array}{l}2.1 \\
3.9\end{array}$ & ${ }_{20}^{11}(8)$ & $\begin{array}{l}1.0(8) \\
1.8\end{array}$ & $\begin{array}{l}1.8(6) \\
3.3\end{array}$ & $\begin{array}{l}28 \\
52\end{array}$ & $\begin{array}{l}0.4(6) \\
0.7\end{array}$ \\
\hline B-4-c & $\mathrm{N}_{2}$ & $\begin{array}{l}\mathrm{a} \\
\mathrm{b}\end{array}$ & $\begin{array}{l}22 \\
22\end{array}(2)$ & $\begin{array}{l}3.5 \\
3.6\end{array}$ & $\begin{array}{l}1.8(1) \\
1.8\end{array}$ & $\begin{array}{l}12(2) \\
12\end{array}$ & $\begin{array}{l}0.9(2) \\
0.9\end{array}$ & $\begin{array}{l}2.6 \\
2.6\end{array}$ & $\begin{array}{l}45 \\
46\end{array}$ & - \\
\hline B-4-e & $\mathrm{N}_{1}$ & $\begin{array}{l}\mathrm{a} \\
\mathrm{b}\end{array}$ & $12^{8}(3)$ & $\begin{array}{l}3.2(3) \\
4.0\end{array}$ & $\begin{array}{l}5.4(3) \\
8.8\end{array}$ & $\begin{array}{l}5 \\
8 \\
8\end{array}$ & $\begin{array}{l}0.5(3) \\
0.9\end{array}$ & $\begin{array}{l}0.8(1) \\
0.9\end{array}$ & - & $\begin{array}{l}0.3(1) \\
0.3\end{array}$ \\
\hline B-5 & $\mathrm{N}_{1}$ & $\begin{array}{l}\mathrm{a} \\
\mathrm{b}\end{array}$ & $\begin{array}{l}17 \\
19^{(2)}\end{array}$ & $\begin{array}{l}5.0(2) \\
5.5\end{array}$ & $\begin{array}{l}1.8(2) \\
1.8\end{array}$ & $\begin{array}{l}15 \\
16\end{array}$ & $\begin{array}{l}1.2(2) \\
1.2\end{array}$ & - & $\begin{array}{l}62 \\
67\end{array}$ & $\begin{array}{l}0.4(1) \\
0.4\end{array}$ \\
\hline Avera & for hole & a & ${ }_{25}^{19}(30)$ & $\begin{array}{l}6.0(33) \\
8.0\end{array}$ & $\begin{array}{l}2.3(30) \\
3.0\end{array}$ & $\begin{array}{l}12 \\
15\end{array}(33)$ & $\begin{array}{l}1.1(32) \\
1.4\end{array}$ & $\begin{array}{l}2.0 \\
2.7\end{array}$ & $\begin{array}{l}56 \\
65\end{array}(14)$ & $\begin{array}{l}1.0(18) \\
0.8\end{array}$ \\
\hline \multicolumn{11}{|c|}{ Site 381} \\
\hline 3 & M-R & $\begin{array}{l}\mathrm{a} \\
\mathrm{b}\end{array}$ & $\begin{array}{l}20 \\
23\end{array}$ & $\begin{array}{l}12 \\
14\end{array}(5)$ & $\begin{array}{l}2.7(6) \\
3.0\end{array}$ & $\begin{array}{l}16 \\
18\end{array}$ & $\begin{array}{l}1.2(6) \\
1.4\end{array}$ & $\begin{array}{l}0.4(6) \\
0.6\end{array}$ & $\begin{array}{l}85 \\
97\end{array}(4)$ & $\begin{array}{l}0.7(6) \\
0.8\end{array}$ \\
\hline 4 & Eopl. & $\begin{array}{l}\mathrm{a} \\
\mathrm{b}\end{array}$ & $\begin{array}{l}10 \\
24\end{array}$ & ${ }_{11}^{4}(1)$ & $\begin{array}{l}1.1 \\
2.7\end{array}$ & $\begin{array}{r}9.5(1) \\
24.0\end{array}$ & $\begin{array}{l}0.3(1) \\
0.7\end{array}$ & $\begin{array}{l}0.5 \\
1.2\end{array}$ & - & - \\
\hline 5 & $\mathrm{~N}_{2}$ & $\begin{array}{l}\mathrm{a} \\
\mathrm{b}\end{array}$ & $\begin{array}{l}22 \\
24\end{array}$ & $\begin{array}{l}11 \\
13\end{array}$ & $\begin{array}{l}2.6(6) \\
2.8\end{array}$ & ${ }_{21}^{18.0_{(4)}}$ & $\begin{array}{l}1.4(6) \\
1.5\end{array}$ & $\begin{array}{l}0.8 \\
0.8\end{array}$ & $\begin{array}{l}58 \\
65\end{array}$ & $\begin{array}{l}0.5(5) \\
0.6\end{array}$ \\
\hline 6 & $\mathrm{~N}_{2}$ & $\begin{array}{l}\mathrm{a} \\
\mathrm{b}\end{array}$ & $\begin{array}{l}18(5) \\
28\end{array}$ & ${ }_{20}^{10}(5)$ & $\begin{array}{l}1.7 \\
2.6\end{array}$ & $10(5)$ & $\begin{array}{l}0.8(4) \\
1.5\end{array}$ & $\begin{array}{l}1.4 \\
2.4\end{array}$ & $\begin{array}{l}57 \\
77\end{array}$ & $\begin{array}{l}0.3 \\
0.5\end{array}$ \\
\hline 7 & $\mathrm{~N}_{2}$ & $\begin{array}{l}\mathrm{a} \\
\mathrm{b}\end{array}$ & $\frac{12}{25}(3)$ & $14^{6}(3)$ & $\begin{array}{l}2.6 \\
4.4\end{array}$ & $16(3)$ & $\begin{array}{l}0.5 \\
1.0\end{array}$ & $\begin{array}{l}0.9 \\
2.4\end{array}$ & $\begin{array}{l}28 \\
60\end{array}$ & $\begin{array}{l}0.4(3) \\
0.7\end{array}$ \\
\hline 8 & $\mathrm{~N}_{1}$ & $\begin{array}{l}\mathrm{a} \\
\mathrm{b}\end{array}$ & $17.5(1)$ & $\begin{array}{l}16(1) \\
17\end{array}$ & $\begin{array}{l}3.6 \\
4.0\end{array}$ & $\begin{array}{l}16_{(1)} \\
18\end{array}$ & $\begin{array}{l}1.3(1) \\
1.4\end{array}$ & - & - & - \\
\hline 9 & $\mathrm{~N}_{1}$ & $\begin{array}{l}\mathrm{a} \\
\mathrm{b}\end{array}$ & $28(2)$ & $12(2)$ & $9.7(1)$ & $\begin{array}{l}18 \\
18\end{array}$ & $\begin{array}{l}1.6 \\
1.6\end{array}(2)$ & $\begin{array}{l}0.5 \\
0.5\end{array}$ & $\begin{array}{l}50 \\
51\end{array}$ & $\begin{array}{l}1.1 \\
1.1\end{array}$ \\
\hline 11 & $\mathrm{~N}_{1}$ & $\begin{array}{l}\mathrm{a} \\
\mathrm{b}\end{array}$ & $\begin{array}{l}16 \\
16\end{array}$ & $\frac{12}{13}(4)$ & $4.1(4)$ & $16(4)$ & $\begin{array}{l}1.4(4) \\
1.4\end{array}$ & $0.6(4)$ & $\begin{array}{l}59 \\
60\end{array}$ & $\begin{array}{l}0.8 \\
0.8\end{array}$ \\
\hline \multicolumn{3}{|c|}{ Average for hole a } & $\begin{array}{l}15 \\
24\end{array}$ & $\begin{array}{l}11(26) \\
15\end{array}$ & $\begin{array}{l}3.1 \\
3.6\end{array}$ & $\begin{array}{l}14(25) \\
18\end{array}$ & $\begin{array}{l}1.2(26) \\
1.4\end{array}$ & $\begin{array}{l}0.8(24) \\
1.3^{2}\end{array}$ & ${ }_{71}^{60}(21)$ & $\begin{array}{l}0.6 \\
0.7\end{array}$ \\
\hline
\end{tabular}

Note: $\mathrm{a}=\mathrm{a}$ natural sediment, $\mathrm{b}=$ on $\mathrm{a}$ carbonate and organic matter free basis. In parentheses is the number of samples. 
TABLE 6

Chemical Composition of Late Quaternary Sediments (\%)

\begin{tabular}{|c|c|c|c|c|c|c|c|c|c|c|c|c|c|c|c|c|c|}
\hline $\begin{array}{l}\text { Horizon } \\
(\mathrm{cm})\end{array}$ & $\mathrm{Mn}$ & $\mathrm{Ni}$ & Co & $\mathrm{Ti}$ & v & $\mathrm{Ca}$ & Mo & $\mathrm{Zn}$ & $\mathrm{Ni}$ & $\mathrm{Cu}$ & $\mathrm{Pb}$ & $\mathrm{Ga}$ & $\mathrm{Be}$ & $\mathrm{Sc}$ & v & $\mathrm{La}$ & $\mathrm{Ba}$ \\
\hline \multicolumn{18}{|c|}{ Station $2291-3$, depth $2151 \mathrm{~m}, 43^{\circ} 11^{\prime} 8 \mathrm{~N}, 36^{\circ} 24^{\prime} 3 \mathrm{E}$} \\
\hline $0-7$ & 0.05 & 0.001 & - & 0.1 & 0.002 & 0.001 & 0.001 & 0.003 & - & 0.001 & 0.001 & 0.001 & - & 0.0001 & 0.002 & 0.003 & 0.03 \\
\hline $9-12$ & & 3 & 0.001 & 1.0 & 02 & 0.008 & 0.001 & 00 & 0.001 & 0.007 & 0.001 & 0.001 & 0.0001 & 0.001 & 0.002 & 0.003 & 0.03 \\
\hline $35-37$ & 0.05 & 0.001 & - & 0.1 & 0.005 & 0.005 & 0.001 & 0.002 & - & 0.003 & 0.001 & 0.001 & - & 0.0002 & 0.001 & 0.002 & 0.05 \\
\hline $43-46$ & 0.06 & 0.01 & 0.001 & 0.5 & 0.001 & 0.01 & 0.001 & 0.01 & 0.001 & 0.003 & 0.001 & 0.001 & 0.0001 & 0.001 & 0.002 & 0.003 & 0.02 \\
\hline $56-59$ & 0.08 & 0.0005 & - & 0.05 & 0.002 & 0.001 & 0.0002 & 0.003 & - & 0.001 & 0.001 & 0.001 & - & 0.0002 & 0.001 & 0.002 & 0.05 \\
\hline $65-66$ & 0.08 & 0.003 & 0.001 & 0.1 & 0.02 & 0.002 & 0.005 & 0.003 & - & 0.002 & 0.001 & 0.001 & - & 0.0005 & 0.002 & 0.005 & 0.05 \\
\hline $100-103$ & 0.08 & 0.005 & 0.001 & 0.7 & 002 & 007 & 0.002 & 0.006 & 0.001 & 0.007 & 0.002 & 0.001 & 0.0001 & 0.001 & 0.002 & 0.002 & 0.05 \\
\hline $132-135$ & 0.08 & 0.007 & 0.001 & 0.7 & 0.02 & 006 & 0.001 & 0.01 & 0.001 & 0.007 & 0.002 & 0.001 & 0001 & .0 & .002 & .002 & 0.05 \\
\hline $152-155$ & 0.1 & 0015 & 0.001 & 0.7 & 0.02 & 0.01 & 0.0002 & 0.005 & 0.001 & 0.008 & & & & & & 3 & 0.03 \\
\hline $185-190$ & 0.08 & 0.007 & 0.001 & 0.7 & 0.02 & 005 & 0.0 & 4 & - & 0.006 & 0.002 & 0.001 & .0001 & .0 & 0.002 & 0.003 & 0.05 \\
\hline $207-202$ & 0.2 & 0.008 & 0.001 & 0.7 & 0.03 & 0.008 & 0.0005 & 0.006 & 0.001 & 0.008 & 0.002 & 0.001 & 0.0001 & 0.001 & 0.002 & 0.003 & 0.03 \\
\hline \multicolumn{18}{|c|}{ Station 2293 , depth $2153 \mathrm{~m}, 43^{\circ} 11^{\prime} 8 \mathrm{~N}, 36^{\circ} 24^{\prime} 2 \mathrm{E}$} \\
\hline $8-10$ & 0.2 & 0.01 & 0.001 & 0.7 & 0.03 & 0.008 & - & 00 & 0.001 & 0 . & 0.002 & 0.001 & te & & 2 & .002 & 0 \\
\hline & & & & & & & - & 5 & 0 & 0. & 2 & 0.0 & 1 & & & & \\
\hline $08-110$ & 0.08 & 0.06 & 0.001 & 0.5 & & & 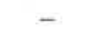 & 0. & 0. & & & & & & 2 & .003 & 0 \\
\hline $158-160$ & 0.08 & 0.005 & 0.001 & 0.5 & 0.02 & 0 & - & 0.002 & 0.001 & 0.0 & 0.002 & 0.001 & 0.0001 & 1 & .002 & 0.002 & 0.0 \\
\hline $208-210$ & 0.08 & 0.005 & 0.001 & 0.5 & ? & 8 & 0.002 & 0005 & 0.001 & & & & & & & $0 \Omega$ & 0.05 \\
\hline $258-260$ & 0.08 & 0.005 & 0.001 & 0.5 & 0.02 & 8 & - & 4 & 0.001 & 0.003 & 0.002 & 0.001 & 0.0001 & 0.0 & 02 & 0.002 & 0.05 \\
\hline $308-310$ & 0.08 & 0.00 & 0.001 & 0.5 & & & 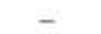 & & - & & & & & & & 0.002 & 0.0 \\
\hline $358-360$ & 0.08 & 0.01 & 0.001 & 0.5 & 0. & 0. & - & 0. & 0.001 & 0.005 & 0.002 & 0.001 & 0.0001 & 0.0 & .002 & 0.002 & 0.0 \\
\hline $378-380$ & 0.08 & 0.005 & 0.001 & 0.4 & & & 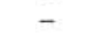 & 0.0 & - & & & & 1 & & & 0.002 & 0.0 \\
\hline $439-443$ & 0.1 & 0.02 & 0.001 & 0. & & & - & & - & 0.001 & & & 0.0001 & & 12 & .002 & 0.0 \\
\hline $490-494$ & 0.1 & 0.01 & 0.001 & 0.5 & & 8 & - & 0. & - & - & 0.0 & & 0.0001 & & & 0.002 & 0.0 \\
\hline $538-542$ & 0.1 & 0. & 0. & 0 . & & & - & & 0.001 & 0.00 & & & & & & .002 & 0.05 \\
\hline $592-596$ & 0.1 & 0.02 & 0.001 & 0.8 & 0.03 & 0.0 & - & 0.0 & 0.001 & 0.0 & 0.002 & 0.001 & 0.00 & & & & 0.03 \\
\hline $645-648$ & 0.1 & 0 & 0 & 0.5 & & & - & & - & & & & 0.0001 & & & & 0.0 \\
\hline $700-703$ & 0.1 & 0.015 & 0.001 & 0.7 & & 0. & - & 0.0 & 0.001 & & & 0.0 & 0.00 & & 0.002 & 0.002 & 0.0 \\
\hline $747-750$ & 0.1 & 0 & 0.001 & 0.5 & & & - & & - & & & & 0.00 & & & 0.002 & 0.0 \\
\hline $797-800$ & 0.1 & 0.008 & 0.001 & 0.5 & 0.02 & 0.01 & 0.0002 & 0.008 & - & 0.006 & 0.002 & 0.001 & 0.0001 & 0.001 & 0.002 & 0.002 & 0.05 \\
\hline
\end{tabular}

TABLE 7

Distribution of Micro-elements in Holocene and Upper Pleistocene Sediments (Station 4752-2)

\begin{tabular}{|c|c|c|c|c|c|c|c|c|c|}
\hline \multirow{2}{*}{$\begin{array}{l}\text { Interval } \\
(\mathrm{cm})\end{array}$} & \multirow[b]{2}{*}{ Type of Sediment } & \multirow[b]{2}{*}{ Age } & & \multirow[b]{2}{*}{$\mathrm{CaCO}_{3}$} & \multirow[b]{2}{*}{$\mathrm{C}_{\text {org }}$} & \multicolumn{4}{|c|}{ Concentration $\left(10^{-4}\right)$} \\
\hline & & & & & & Mo & Co & $\mathrm{Ni}$ & $\mathrm{V}$ \\
\hline $0-25$ & Clay low calcareous microlaminated & $\mathrm{Hl}_{\mathrm{III}}$ & a & 24.6 & 2.21 & 7.0 & 28 & 100 & 200 \\
\hline $25-33$ & Clay & $\mathrm{Hl}_{U}$ & a & - & - & 3.0 & 25 & 120 & $\begin{array}{l}282 \\
220\end{array}$ \\
\hline $33-48$ & Clay & $\mathrm{Hl}_{\mathrm{III}}$ & $\mathrm{a}$ & - & - & 5.0 & 24 & 100 & 140 \\
\hline \multirow[t]{2}{*}{$48-54$} & \multirow[t]{2}{*}{ Clay low calcareous microlaminated } & \multirow[t]{2}{*}{$\mathrm{Hl}_{\mathrm{II}}$} & $\mathrm{a}$ & 14.2 & 1.31 & 4.8 & 17 & 77 & 100 \\
\hline & & & b & - & - & & & 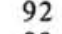 & 120 \\
\hline \multirow{2}{*}{$54-58$} & \multirow{2}{*}{ Clay low calcareous microlaminated } & \multirow{2}{*}{$\mathrm{HI}_{\mathrm{II}}$} & a & 14.1 & 1.02 & 5.0 & 23 & 90 & 190 \\
\hline & & & b & - & - & 6.0 & 27 & 107 & 226 \\
\hline \multirow[t]{2}{*}{$163-168$} & \multirow{2}{*}{ Clay low calcareous microlaminated } & \multirow[t]{2}{*}{$\mathrm{Hl}_{\mathrm{II}}$} & a & 15.3 & 1.33 & 2.0 & 13 & 60 & 90 \\
\hline & & & b & - & - & 2.4 & 16 & 73 & 110 \\
\hline \multirow[t]{2}{*}{$168-172.5$} & \multirow{2}{*}{$\begin{array}{l}\text { Clay low calcareous enriched in } \\
\text { organic matter }\end{array}$} & \multirow{2}{*}{$\mathrm{Hl}_{\mathrm{II}}$} & a & 12.2 & 4.29 & 7.2 & 28 & 96 & 130 \\
\hline & & & b & - & - & 9.1 & 35 & 121 & 164 \\
\hline \multirow[t]{2}{*}{$190-198.5$} & \multirow{2}{*}{$\begin{array}{l}\text { Clay low calcareous enriched in } \\
\text { organic matter }\end{array}$} & \multirow{2}{*}{$\mathrm{Hl}_{\mathrm{Il}}$} & a & 21.7 & 3.56 & 2.2 & 23 & 85 & 100 \\
\hline & & & b & - & 4.5 & 3. & 32 & 119 & 140 \\
\hline \multirow[t]{2}{*}{$198.5-210$} & \multirow[t]{2}{*}{ Sapropel-clay microlaminated } & \multirow[t]{2}{*}{$\mathrm{Hl}_{\text {II }}$} & a & 11.7 & 8.76 & 7.5 & 25 & 64 & 80 \\
\hline & & & b & - & 9.92 & 10.6 & 35 & 90 & 113 \\
\hline \multirow[t]{2}{*}{$210-212$} & \multirow[t]{2}{*}{ Sapropel-clay microlaminated } & \multirow[t]{2}{*}{$\mathrm{Hl}_{\mathrm{II}}$} & a & 13.7 & 5.25 & 5.0 & 23 & 90 & 200 \\
\hline & & & b & - & 6.08 & 6.6 & 30 & 119 & 264 \\
\hline \multirow[t]{2}{*}{$260-279$} & \multirow[t]{2}{*}{ Clay low calcareous } & \multirow{2}{*}{$\mathrm{Hl}_{\mathrm{I}}$} & a & 15.8 & 1.20 & 0.5 & 26 & 88 & 180 \\
\hline & & & b & - & 1.40 & 0.6 & 32 & 108 & 220 \\
\hline $115-120$ & Clay low calcareous & $\mathrm{W}_{\mathrm{II}}$ & a & 20.69 & 0.50 & - & 24 & 68 & 240 \\
\hline & & & b & - & 0.00 & - & 31 & 87 & 306 \\
\hline
\end{tabular}

Note: $\mathrm{a}=$ in natural dry sediment, $\mathrm{b}=$ on a carbonate, organic matter $\left(\mathrm{C}_{\text {org }} \times 2\right)$ free basis.

(negatively) with quantitative changes in $\mathrm{CaCO}_{3}$ contents. Thus, a true pattern of relatively sedimentary $\mathrm{Fe}, \mathrm{Mn}$, and $\mathrm{Ti}$ concentrations is distorted by a clearly non-uniform diluting influence of carbonates, which also affects the distribution of most micro-elements. The micro-element contents are similar in non- calcareous and low-calcareous muds, and in analogous deposits of other units.

Unit B-4-a (Pliocene): In contrast to Unit B-2 and B3 deposits, the section here is mainly composed of noncalcareous clays, often rather finely dispersed (over $90 \%$ in the $<0.01 \mathrm{~mm}$ fraction). These muds are similar to 
TABLE 8

Mineral Composition of Upper Quaternary Black Sea Sediments (X-ray diffraction analysis)

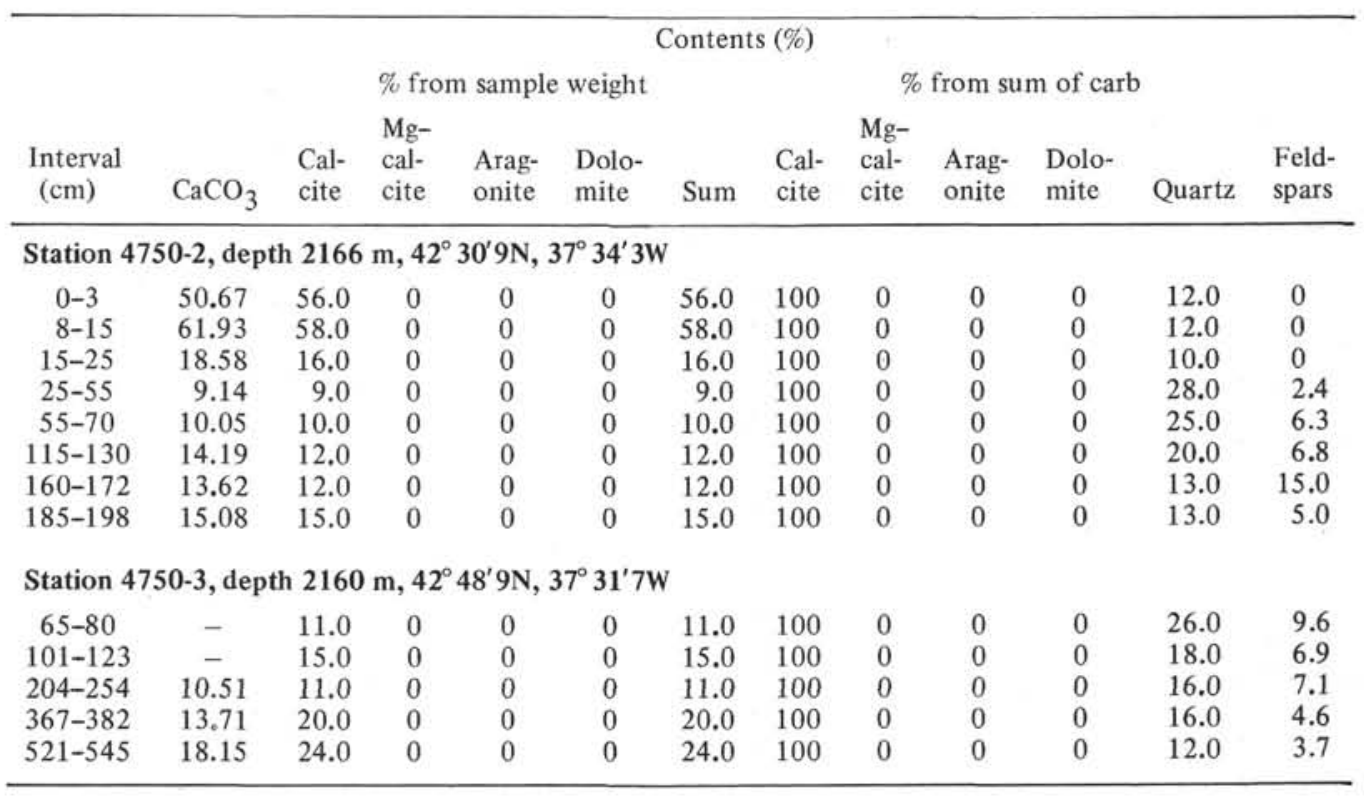

Unit B-3 sediments in clay material composition. The $\mathrm{CaCO}_{3}$ content is extremely low, rarely exceeding $1-2 \%$. Corg contents vary widely $(0.67-1.85 \%)$.

Characteristic components of this unit are interbeds of relatively pure siderite, marly sediments with a siderite admixture, and diatomaceous oozes. Relative enrichment in diatoms is common in interbeds with increased contents of organic matter. Though amorphous silica content is relatively low in these oozes (3.39-4.72\%), two interbeds $(41-4,10-20 \mathrm{~cm}$, and $41-6$, $108-122 \mathrm{~cm}$ ) are quite enriched (15.62-18.99\%), and are low-siliceous diatomaceous oozes. A relatively higher content of amorphous silica $(6.35 \%)$ is found in a single interbed poor in organic matter. The $\mathrm{Fe}, \mathrm{Mn}$, and $\mathrm{Ti}$ contents in those oozes enriched in organic matter and diatoms vary little from layer to layer, and are similar to amounts seen in terrigenous muds of other units. They are lower in low-siliceous diatomaceous muds.

One of the siderite beds studied $(40-2,148-149 \mathrm{~cm})$ in enriched in $\mathrm{Fe}(35.50 \%)$ and $\mathrm{Mn}(3.65 \%)$ and impoverished in $\mathrm{Ti}(0.12 \%)$.

Certain micro-elements ( $\mathrm{Li}, \mathrm{Rb}, \mathrm{Cs}, \mathrm{Pb}, \mathrm{Sn}, \mathrm{F}, \mathrm{B})$ were studied, mainly from those beds enriched in organic matter. Although low in diatomaceous muds, in other mud types their contents are similar to those found in analogous facies types in other units.

Unit B-4-b (Pliocene) consists of non-calcareous and low-calcareous clay and silty-clay and diatomaceous muds with widely varying carbonate contents. In the lower part of the unit (Cores 48-54), calcareous (32.58$50.11 \% \mathrm{CaCO}_{3}$ ) and high-calcareous (up to $73.27 \%$ $\left.\mathrm{CaCO}_{3}\right)$ muds prevail. Non-calcareous (0.88-1.0\% $\left.\mathrm{CaCO}_{3}\right)$ and low-calcareous (14.76-23.50\% $\left.\mathrm{CaCO}_{3}\right)$ clay interbeds occur. These muds and oozes are similar to the overlying unit in clay composition. Besides calcite, a siderite admixture sometimes occurs in the carbonate fraction. The upper part of the unit (Cores 43-47) is enriched in organic matter (1.22-3.18\% Corg). $\mathrm{CaCO}_{3}$ and amorphous silica have wide ranges of values in these muds and oozes $(0.0-30.80 \%$ and $7.01-14.80 \%$, respectively); $\mathrm{Fe}, \mathrm{Mn}$, and $\mathrm{Ti}$ contents slightly exceed the clarke. The same micro-element group as in Unit B4-a was studied; their contents are similar to those of corresponding deposits of other units. Quantitative variations are insignificant and, to a certain extent, are correlated negatively with $\mathrm{CaCO}_{3}$ content. There is a marked increase of the $\mathrm{Rb}$ contents in an interbed of non-calcareous, low-siliceous diatomaceous mud (43-1, $0-13 \mathrm{~cm})$.

In the lower part of the unit (Cores 48-54), a low organic matter content $(0.8-0.64 \%$ of $\mathrm{Corg})$ is characteristic. A single bed of calcareous aleurite-pelitic mud contains $1.23 \%$ Corg $(52-6,121-123 \mathrm{~cm})$. Content of amorphous silica is also low (3.73\%).

Though $\mathrm{Fe}, \mathrm{Mn}$, and $\mathrm{Ti}$ only slightly exceed the clarke $(\mathrm{Kb})$, they are subject to significant quantitative variations in the lower portion of the unit. They primarily show a negative correlation with carbonate content in sediments and have an opposite sign, i.e., a dilution of clay material by carbonates.

The same group of micro-elements $(\mathrm{Li}, \mathrm{Rb}, \mathrm{Cs}, \mathrm{Pb}$, Sn, F, B) was studied as in the preceding unit. Insignificant quantitative variations are linked with irregular distribution of carbonates in the sediments. Relatively increased $\mathrm{Rb}$ contents are observed in some beds of calcareous oozes poor in organic matter.

The lower part of the Pliocene sequence (Unit B-4-e) is represented by finely laminated clay sediments, the carbonate contents of which range from 0.88 to 4.688 percent, and which have a low content of organic matter $\left(0.58-0.64 \%\right.$ of $\left.\mathrm{Corg}_{\text {o }}\right)$ and amorphous silica. Only in one interbed $(56-1,95-105 \mathrm{~cm})$ are the $\mathrm{SiO}_{2}$ amorph contents increased $(12.29 \%)$. This bed may be called a diatomaceous low-siliceous mud. The Fe, Mn, and Ti contents are close to the clarke (Table 13).

Unit B-4-e (Miocene) is represented by compact clays, often aleuritic, alternating with marly oozes (34.62-50.0\% $\mathrm{CaCO}_{3}$ ) (carbonate varves). 


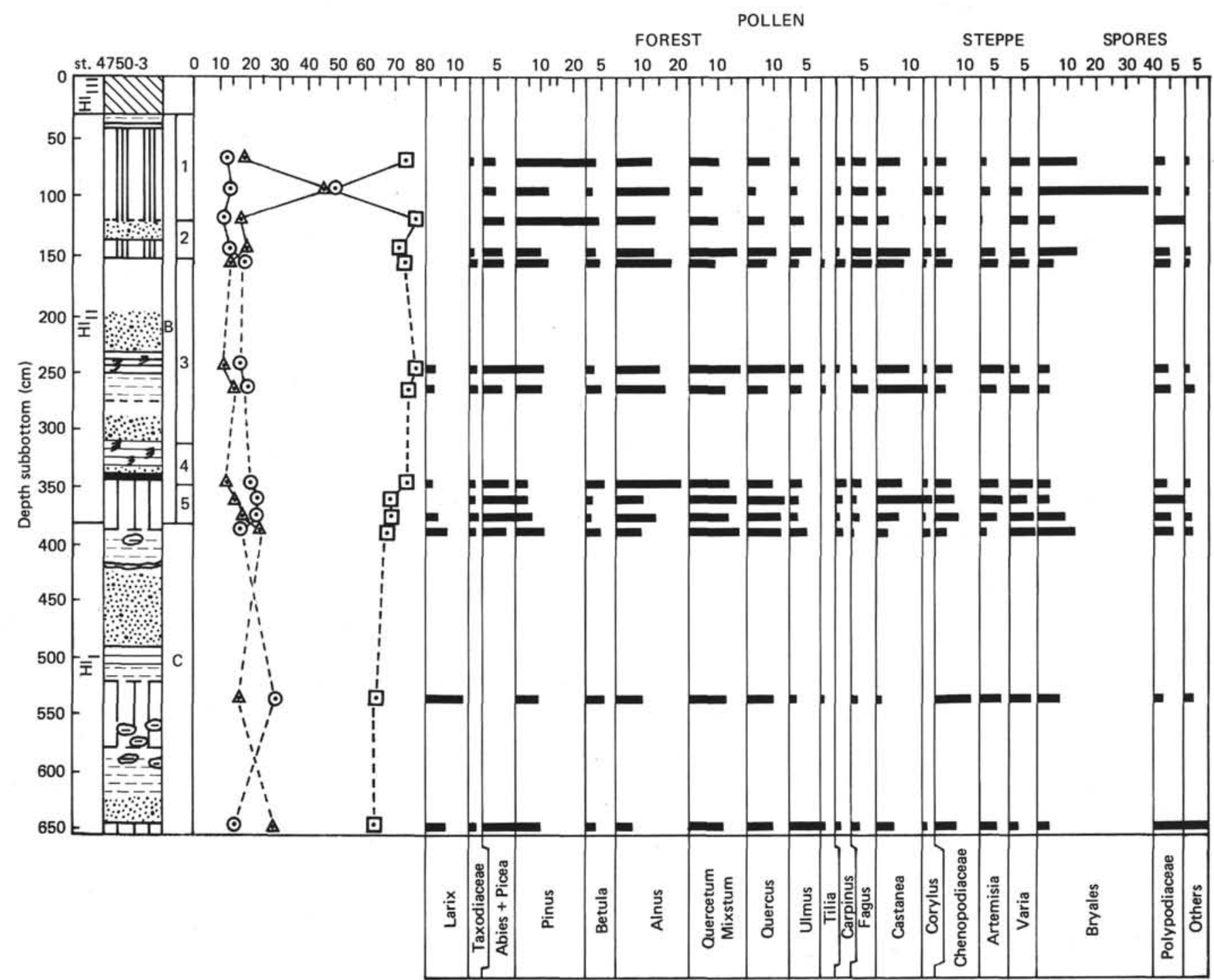

Figure 5. Lithology and spore-pollen of late Quaternary sediments in Core 4750-3 (data by Komarov). Graphic symbols as in Figure 8.

Illite dominates the clay fraction and montmorillonite and chlorite are present almost in equal amounts; kaolinite occurs sporadically. Dolomite is often a common carbonate mineral, with marked aragonite and calcite admixtures.

Clays and marly oozes contain little organic matter (0.11-0.65\% Corg) and amorphous silica $(1.20-2.12 \%)$. The $\mathrm{Fe}, \mathrm{Mn}$, and Ti contents are within the clarke (Ko) and vary inversely with $\mathrm{CaCO}_{3}$. The $\mathrm{Fe}, \mathrm{Mn}$, and $\mathrm{Ti}$ contents approach the clarke in the interbeds of dolomitized limestones, ankerites, and dolomites (64-4, $20-22 \mathrm{~cm}, 65-2,52-56 \mathrm{~cm}, 69-3,72-74 \mathrm{~cm})$. Compared to surrounding sediments, the Mn contents are higher and the $\mathrm{Ti}$ ones are lower. Quantitative variations of the studied micro-elements $(\mathrm{Li}, \mathrm{Rb}, \mathrm{Cs}, \mathrm{Pb}, \mathrm{Sn}, \mathrm{F}, \mathrm{B})$ are negligible. Relatively high $\mathrm{Pb}$ contents are clearly seen in carbonate muds from the upper part of the unit, being nearly the same as in the interbeds of completely non-calcareous sediments. Moreover, the $\mathrm{Rb}$ and $\mathrm{Pb}$ contents are higher throughout the section than those of the lower portion of Unit B-4-b.
Unit B-5 consists of black compact clays ("black shales") and interbeds of tuffitic sandstones, dolomites, and rhodochrosite(?).

Clays, often aleuritic, are poor in carbonates (3.78$\left.5.51 \% \mathrm{CaCO}_{3}\right)$, organic matter $(0.16-0.46 \%$ Corg $)$ and amorphous silica (1.09-2.67\%). The clay is mostly illite with some montmorillonite and little chlorite and kaolinite. $\mathrm{Fe}, \mathrm{Mn}$, and $\mathrm{Ti}$ contents are close to the clarkes, with small ranges. The $\mathrm{Fe}$ and $\mathrm{Ti}$ contents are low where the $\mathrm{Mn}$ content is relatively increased in the dolomite $(70-5,104-222 \mathrm{~cm}, 73-1,58-60 \mathrm{~cm})$ and rhodochrosite(?) (70-3, 104-107 cm) interbeds. Microelements ( $\mathrm{Li}, \mathrm{Rb}, \mathrm{Cs}, \mathrm{Pb}, \mathrm{Sn}, \mathrm{F}, \mathrm{B})$ occur in the clays in the same amounts as in corresponding Unit B-4-e, but are lower to a certain degree in marly oozes, due to carbonate dilution.

The general geochemical characteristics of sediments from Hole $380 \mathrm{~A}$ are as follows:

1) Using average values (Table 14), the upper part of the Pliocene sediments are most enriched in organic matter and $\mathrm{SiO}_{2}$ amorph (Units B-4-a, B-4-b). 


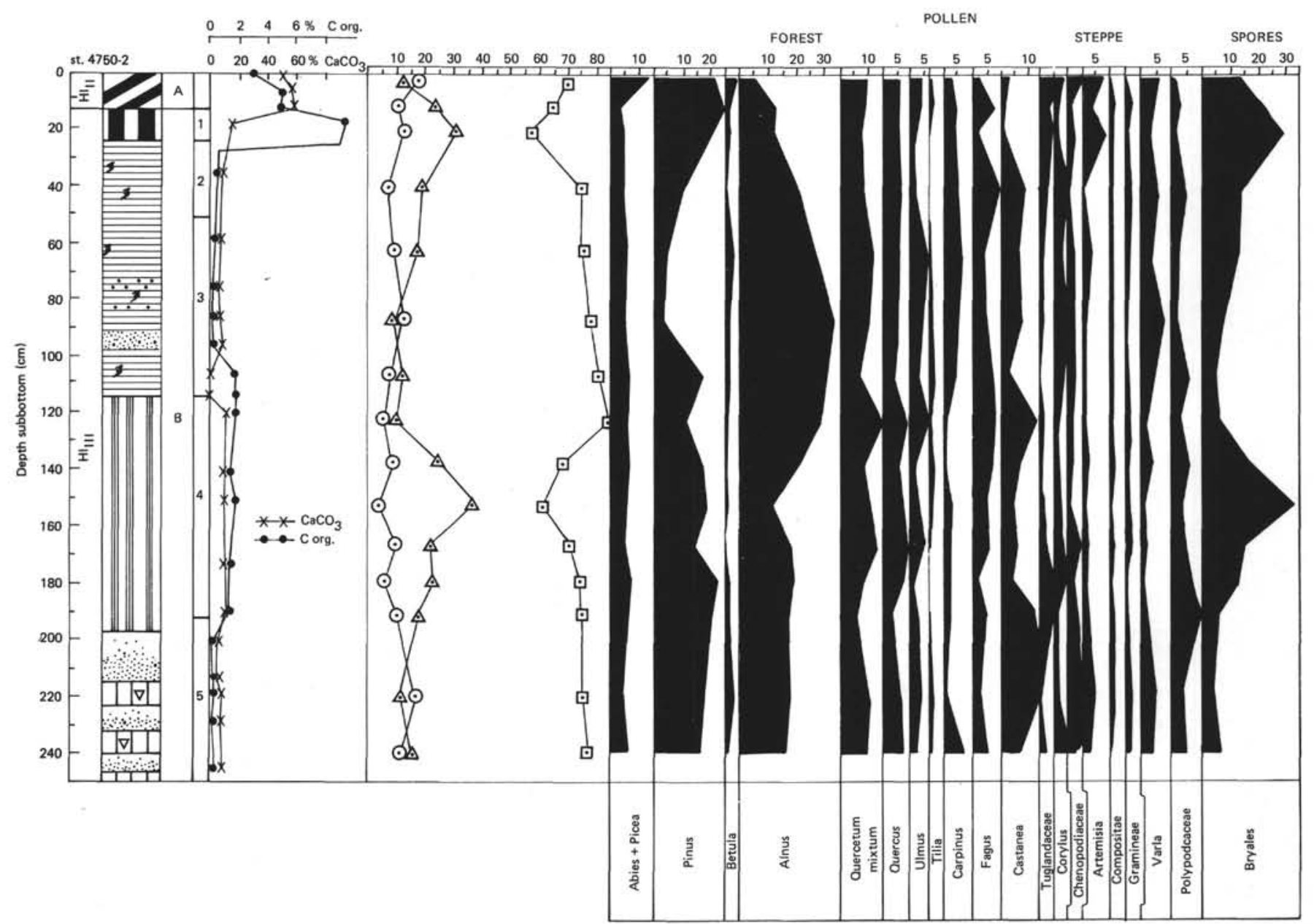

Figure 6. Lithology and spore-pollen of late Quaternary sediments in Core 4750-2 (data by Komarov). Graphic symbols as in Figure 8.

Maximum $\mathrm{Fe}$ and $\mathrm{Mn}$ contents are also found here, in the siderite interbeds. Phosphorus is highest in Unit B2 (Eopleistocene). Miocene sediments (B-4-e) show slightly increasing $\mathrm{Rb}$ contents. The average contents of $\mathrm{F}$ are highly variable down the section, but are somewhat higher in Eopleistocene sediments (B-2, B-3).

Quantitative variations of $\mathrm{Li}, \mathrm{Cs}, \mathrm{Rb}$, and $\mathrm{Sn}$ are insignificant, although a considerable difference exists in $\mathrm{Na}$ content between Units $\mathrm{B}-2$ and $\mathrm{B}-3$ in the Eopleistocene section. The $\mathrm{Zn}, \mathrm{Ni}, \mathrm{Cr}, \mathrm{Li}$, and $\mathrm{F}$ contents, on a carbonate-free basis, are higher in Unit B-3.

2) No regular variations with sediment types occur for most micro-elements. A certain decrease in contents of micro-elements is observed with increasing carbonate and siliceous contents.

3) The ratios of average element contents to clarkes are distinctive for individual units. As a rule, average $\mathrm{Fe}$ and $\mathrm{Mn}$ contents are higher than that of Ko. They exceed Ko markedly in the upper Pliocene deposits (B4-a), as a result of occurrence of siderite and, possibly, rhodochrosite. Average contents of other elements are close to Ko (more often lower).

4) The Ti, P, Li, Rb, and Sn contents of Neogene sediments are considerably lower than the clarkes of sedimentary rocks ( $\mathrm{Rb}-$ sharply, $\mathrm{Sn}-$ manyfold). $\mathrm{Fe}$ and $\mathrm{Mn}$ contents exceed Ko and are the exceptions. The amounts of most studied elements are close to their contents in intermediate and acid rocks. This suggests an intensive accumulation of erosion products of intermediate and acid rock massifs (as in Hole 380).

\section{Site 381}

Lithologically, this site was subdivided into 11 units dating from Pleistocene to Neogene; Units 3-11 were studied. The lithologic subdivision is confirmed by our shore laboratory chemical analyses (Figures 13, 14).

Unit 3 (M-R and M) mainly consists of very fine terrigenous clay muds (up to $92.12 \%$ in the $0.01 \mathrm{~mm}$ fraction). Regularly high contents of clay minerals (montmorillonite and illite) as well as low quartz and feldspar contents are characteristic of this unit. The $\mathrm{CaCO}_{3}$ content is variable (from 2.30 to $36.50 \%$ ), increasing from the top to the bottom. $\mathrm{C}_{\text {org }}$ content is generally low ( $0.47 \%$ average) and decreases from top to bottom. Amorphous silica is usually in small amounts $(<3.0 \%)$ and increases at the bottom of the unit to $5.25 \%$.

Average $\mathrm{Fe}, \mathrm{Ti}$, and $\mathrm{Mn}$ contents, like those of some other elements, are higher as compared with their 

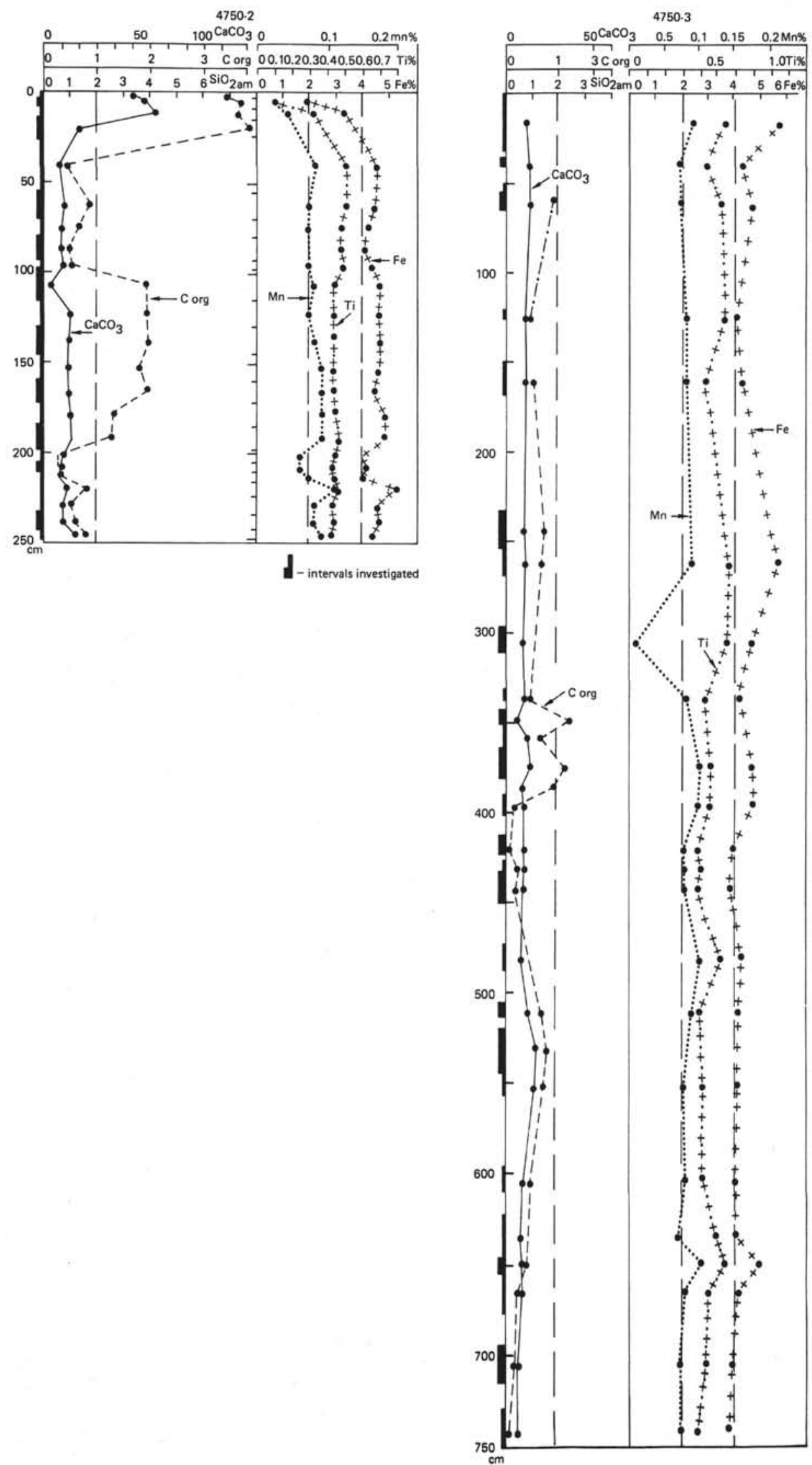

Figure 7. Chemical macrocomponent distribution in late Quaternary sediments at Stations 4750-2 and 4750-3 (in natural sediments). Stratigraphy and lithology as in Figures 5 and 6. 

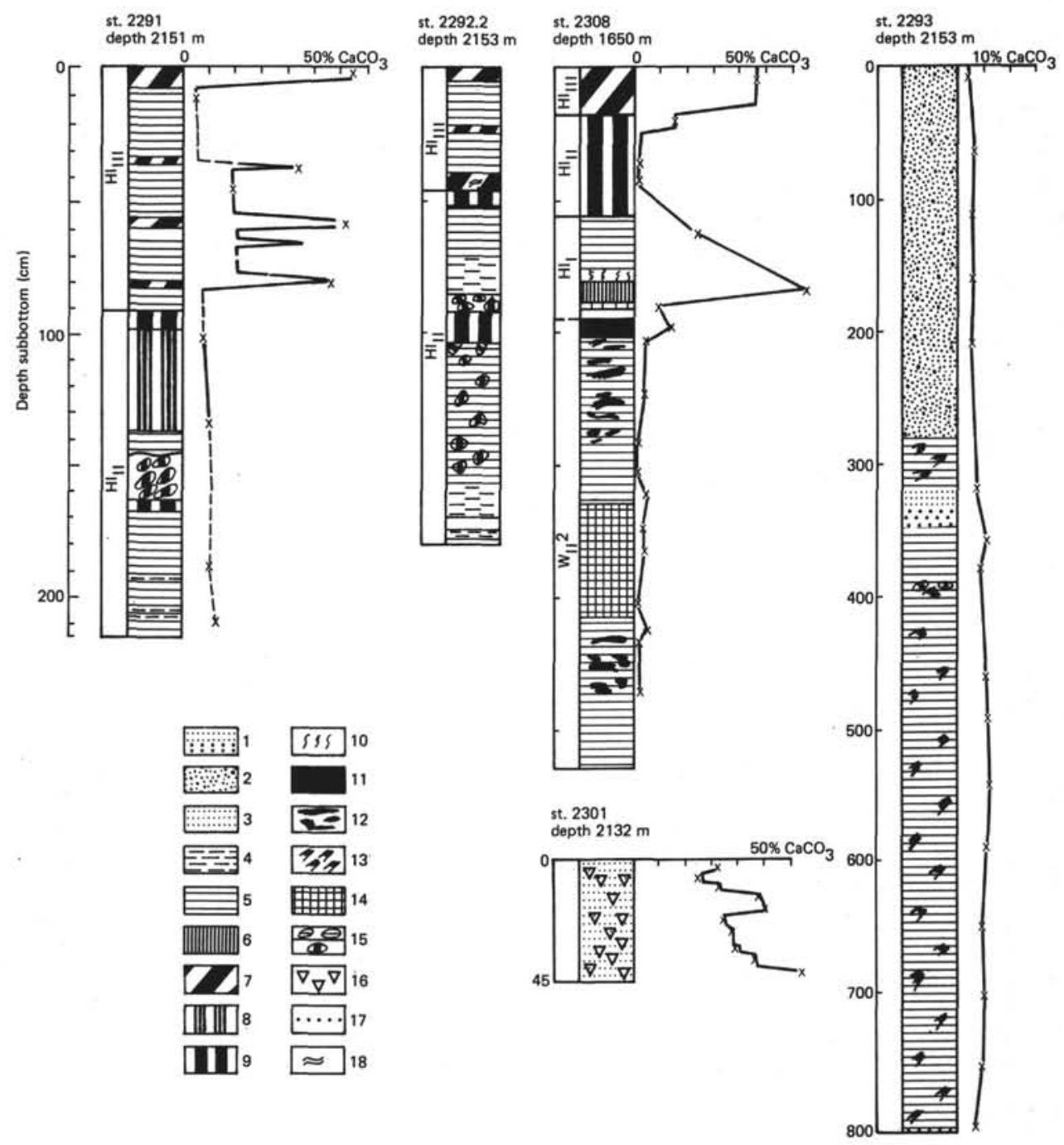

Figure 8. Lithological composition of late Quaternary sediments from stations taken in the Site 379 region. (1) clastic turbidites; (2) clastic non-calcareous sands; (3) clastic non-calcareous coarse silts; (4) clastic non-calcareous fine silts; (5) non-calcareous $\left(<10 \%\right.$ of $\left.\mathrm{CaCO}_{3}\right)$ and low-calcareous (10-30\% of $\left.\mathrm{CaCO}_{3}\right)$ clay muds, gray, dark-gray; (6) calcareous clay and calcareous muds (30-50\% $\left.\mathrm{CaCO}_{3}\right)$ poor in organic matter (bluish-gray); (7) microlaminated nannofossil muds (>50\% $\mathrm{CaCO}_{3}, 3-5 \% \mathrm{C}_{\text {org }}$ ); (8) clay and aleurite-clay muds, microlaminated and non calcareous (1.5-2.0\% Corg); (9) sapropel-clay and sapropelic muds (10-30\% and more organic matter), non-calcareous, microlaminated (greenish brown, brown); (10) diatom-clay muds, low-siliceous (10-30\% $\mathrm{SiO}_{2}$ amorph); (11) hydrotroilitic-clay non-calcareous and low-calcareous muds, black; (12) interbeds of hydrotroilite-clay muds uneven in thickness; (13) hydrotroilite gouges; (14) clays, non calcareous, brownish, banded-mottled (the alternation of gray, red, and brown bands); (15) sediments of lentiform texture: (a) lenses of uniform clay mud; (b) the same of sapropelic, sapropel-clay mud; (16) shell detritus; (17) interbeds of clastic aleurite of small thickness; (18) break of occurrence.

clarkes in modern marine sediments. A decrease in $\mathrm{Fe}$, $\mathrm{Ti}$, and some minor elements toward the bottom results from increasing $\mathrm{CaCO}_{3}$ content (Tables 9, 15, 16; Figure 13).

Unit 4 (Eopleistocene) differs markedly from Unit 3 geochemically; the unit sediments are coarser (sands, coarse aleurites) and more calcareous, containing much quartz and feldspars and authigenic carbonates, and only a small amount of clay minerals, The $\mathrm{SiO}_{2}$ amorph and Corg contents are a bit lower than those of the base of Unit 3.

The contents of all studied elements, excluding $\mathrm{Mn}$, are lower in quartz sands $(18-2,0-15 \mathrm{~cm})$. Abundant quartz suggests formation by rapid accumulation of shallow water material (turbidites).

The $\mathrm{CaCO}_{3}, \mathrm{Fe}$, and $\mathrm{Mn}$ contents are markedly increased in the calcareous micrite interbed (calcareous fine aleuritic ooze, Sample 19-1, 0-15 cm), 56.78\%, 
TABLE 9

Average Contents of Elements in Volcanic and Sedimentary Rocks of the Earth's Crust (according to the clarkes of Vinogradov, 1962) and Bottom Sediments of Different Regions of the Atlantic Ocean Basin

\begin{tabular}{|c|c|c|c|c|c|c|c|c|c|c|c|c|c|c|c|c|}
\hline \multirow[b]{2}{*}{$\begin{array}{l}\text { Rock, Type of } \\
\text { Sediment }\end{array}$} & \multirow[b]{2}{*}{$\begin{array}{l}\text { Region of } \\
\text { the Basin }\end{array}$} & \multicolumn{9}{|c|}{ Content $(\%)$} & \multicolumn{6}{|c|}{ Content $\left(10^{-4} \%\right)$} \\
\hline & & $\mathrm{CaCO}_{3}$ & $\mathrm{SiO}_{2}$ & $\mathrm{C}_{\text {org }}$ & $\mathrm{Fe}$ & $\mathrm{Mn}$ & $\mathrm{Ti}$ & $P$ & K & $\mathrm{Na}$ & $\mathrm{Ba}$ & $\mathrm{Zr}$ & $\mathrm{Cu}$ & $\mathrm{Zn}$ & $\mathrm{Ni}$ & Co \\
\hline Ultrabasic $\left(\mathrm{K}_{\mathrm{u}}\right)$ & Earth crust & - & - & - & 9.85 & 0.15 & 0.03 & 0.02 & 0.03 & 0.57 & 1 & 30 & 20 & 30 & 2000 & 210 \\
\hline Basic $\left(K_{b}\right)$ & Earth crust & - & - & - & 8.56 & 0.20 & 0.90 & 0.14 & 0.83 & 1.94 & 300 & 100 & 100 & 130 & 160 & 45 \\
\hline Intermediate $\left(\mathrm{K}_{\mathrm{i}}\right)$ & Earth crust & - & - & - & 5.85 & 0.12 & 0.80 & 0.16 & 2.30 & 3.00 & 650 & 260 & 35 & 72 & 55 & 10 \\
\hline $\begin{array}{l}\text { Granites, } \\
\quad \text { granodiorites }\left(\mathrm{K}_{\mathrm{a}}\right)\end{array}$ & Earth crust & - & - & - & 2.70 & 0.06 & 0.23 & 0.07 & 3.34 & 2.77 & 830 & 200 & 20 & 60 & 8 & 5 \\
\hline Clays, shales $\left(\mathrm{K}_{\mathrm{o}}\right)$ & Earth crust & - & - & - & 3.33 & 0.07 & 0.45 & 0.08 & 2.28 & 0.66 & 800 & 200 & 57 & 60 & 95 & 20 \\
\hline \multicolumn{17}{|c|}{ Bottom Sediments (layer $0-5 \mathrm{~cm}$ ) } \\
\hline $\begin{array}{l}\text { Terrigenous sediments } \\
\quad \text { (layer } 0-5 \mathrm{~cm} \text { ) }\end{array}$ & Baltic Sea ${ }^{a}(1)$ & 1.14 & 1.87 & 1.66 & 2.99 & 0.08 & 0.42 & 0.06 & - & - & 810 & 270 & 33 & 83 & 31 & - \\
\hline & Black Sea $^{{ }^{(2)}}$ & $\begin{array}{r}0.73 \\
12.30\end{array}$ & 2.33 & $\begin{array}{l}3.32 \\
1.00\end{array}$ & $\begin{array}{l}4.25 \\
3.25\end{array}$ & $\begin{array}{l}0.05 \\
0.07\end{array}$ & $\begin{array}{l}0.39 \\
-\end{array}$ & $\begin{array}{l}0.07 \\
0.05\end{array}$ & $\overline{-}$ & $\overline{-}$ & $\begin{array}{l}820 \\
210\end{array}$ & $\begin{array}{r}280 \\
54\end{array}$ & $\begin{array}{l}46 \\
34\end{array}$ & 155 & $\begin{array}{l}35 \\
44\end{array}$ & $\overline{11}$ \\
\hline Average for all types & Atlantic Ocean $\mathrm{c}(1)$ & - & 2.80 & 1.09 & 3.05 & 0.10 & 0.31 & 0.10 & - & - & 450 & 160 & 54 & - & 35 & - \\
\hline $\begin{array}{l}\text { of sediments }(352-960 \\
\text { samples, layer } 0-5 \mathrm{~cm})\end{array}$ & & - & 3.79 & 1.67 & 4.88 & 0.19 & 0.50 & 0.21 & - & - & 780 & 270 & 109 & - & 39 & - \\
\hline
\end{tabular}

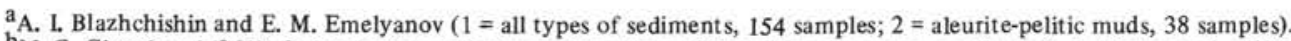

${ }_{\mathrm{M}}$. G. Glagoleva ( 1961). Only aleurites and a leuropelites (layer 0-5 cm) (macrocomponents), A. E. Babinetz et al., 1973, p. 146. Late Quaternary sediments (layer $0-9 \mathrm{~cm}$ ), (microelements).

c According to the different data of E. M. Emelyanov.

$9.66 \%$, and $0.22 \%$, respectively, whereas the $\mathrm{Ti}$ and other elements associated with clays are lowered. Authigenous ferruginous carbonates (siderite, ankerite) occur in the micrite causing high $\mathrm{Fe}$ and $\mathrm{Mn}$ contents. facies, alternating as clearcut laminae, provide both a

Unit $5\left(\mathrm{~N}_{2}\right)$ is represented by diatomaceous muds, with interbeds of clay muds and sapropels. These facies, alternating as clearcut laminae, provide both a peculiar chemical composition and element distribution in the unit whose sediments consist of pelitic and aleurite-pelitic muds containing 48.56 to $91.18 \%$ in the $<0.01 \mathrm{~mm}$ fraction. Lower contents of feldspars $(<10 \%)$ and quartz $(<20 \%)$ and extremely irregular distribution of clay minerals are characteristic of the unit.

The $\mathrm{CaCO}_{3}$ contents range from 0.0 to $42.25 \%$; $\mathrm{SiO}_{2}$ amorph and $\mathrm{C}_{\text {org }}$ contents are high, to 1.70 and $8.67 \%$, respectively. The beds of low-siliceous diatomaceous oozes, enriched in organic matter, may thus be easily identified.

Two carbonate interbeds in the sediments of this unit contain the highest $\mathrm{Fe}(19.55$ and $35.00 \%)$ and $\mathrm{Mn}$ $(1.84 \%$ and $5.43 \%)$ contents recorded in the Black Sea. Diffractometric and immersion analyses, made in addition to petrographic studies of thin sections, showed a clear predominance of chemogenic-diagenetic carbonate, basically siderite. Manganous carbonates are likely to be present here as well: a carbonate-like manganosiderite is found in Core 27, according to the shipboard data $35.0 \% \mathrm{Fe}$ and $5.43 \% \mathrm{Mn}$ occur in this core). Of the micro-elements, $\mathrm{V}, \mathrm{Cr}, \mathrm{Ni}$, and $\mathrm{Co}$ are markedly enriched in Unit 5 (Table 16).

Unit $6\left(\mathrm{~N}_{2}\right)$ is represented by laminated diatom micrites (basically pelitic and aleurite-pelitic muds, $67.65-78.69 \%$, which is in the $<0.01 \% \mathrm{~mm}$ fraction) with interbeds of terrigenous muds enriched in diatoms. Irregularity of chemical and mineral composition (mainly clay minerals and quartz) is, in general, typical of this unit. The $\mathrm{CaCO}_{3}$ and $\mathrm{C}_{\text {org }}$ contents vary within the range $1.85-70.5 \%$ and $0.48-5.88 \%$, respectively. The
$\mathrm{SiO}_{2}$ amorph contents are lower, as a whole, than in Unit 5. Clearcut lamination of sediments are the varying $\mathrm{CaCO}_{3}, \mathrm{SiO}_{2}$ amorph, and $\mathrm{Corg}$ contents cause a highly irregular distribution of different metals as well: $\mathrm{Fe}$, $\mathrm{Mn}, \mathrm{Ti}$, etc. (Table 15, Figure 13). In general, the contents of these metals are low in natural sediments (averaging to $2.97,0.07$, and $0.30 \%$, respectively).

Unit $7\left(\mathrm{~N}_{2}\right)$, unlike other units, is represented by aragonite micrites with terrigenous mud, sapropel, and diatomaceous mud interbeds. By grain size, these are principally aleurite-pelitic muds containing 4.00 to $81.84 \% \mathrm{CaCO}_{3}$. Characteristics of this unit are: (1) high $\mathrm{C}_{\text {org }}$ contents (up to $6.56 \%$ ), mostly in inverse correlation with $\mathrm{CaCO}_{3}$ contents; (2) high $\mathrm{SiO}_{2}$ amorph contents (up to $8.28 \%$ ). $\mathrm{Fe}, \mathrm{Mn}, \mathrm{Ti}$, and minor element contents are low; in general, distribution is extremely irregular. The aragonitic mineralogy of the needle-like and "rice grain" crystals reported by shipboard geologists is confirmed by X-ray analysis. Fe and $\mathrm{Mn}$ contents (i.e., typical of carbonaceous sediments) are low in contrast to Unit 5.

Unit $8\left(\mathrm{~N}_{1}\right)$ is represented by laminated compact clays (black shales), breccias, and sandstones with carbonate (ankerite-dolomite) cement. Sandstones consist mostly of quartz, with some feldspars. A few breccia and sandstone rocks have been analyzed. The $\mathrm{C}_{\text {org }}, \mathrm{Fe}, \mathrm{Mn}$, and $\mathrm{Ti}$ contents are low, especially that of $\mathrm{Fe}(0.98-1.38 \%)$, which is high only in a single sample (42-1, 140-145 cm).

Unit $9\left(\mathbf{N}_{1}\right)$ consists of laminated compact, often aleuritic, clays (black shales). Illite and chlorite are dominant; montmorillonite, with some kaolinite are present. Feldspar content exceeds that of quartz $(\mathrm{Q} / \mathrm{Fp}<1)$ suggesting that sedimentary material was supplied from the folded mountain drainage area. Clays contain almost no carbonates $(0.77-1.00 \%$ $\left.\mathrm{CaCO}_{3}\right)$, have considerable amounts of $\mathrm{SiO}_{2}$ amorph (1.98$2.08 \%)$, and have $\mathrm{C}_{\text {ors }}(0.71-1.12 \%)$ and metal contents typical of terrigenous muds. 
TABLE 9-Continued

Content $\left(10^{-4} \%\right)$

\begin{tabular}{|c|c|c|c|c|c|c|c|c|c|c|c|c|c|c|c|c|c|c|c|c|c|}
\hline $\mathrm{Cr}$ & V & Sn & $\mathrm{Pb}$ & Mo & W & $\mathbf{L i}$ & $\mathrm{Rb}$ & Cs & $\mathrm{Ag}$ & $\mathrm{F}$ & B & $\mathrm{Cd}$ & $\mathbf{L a}$ & $\mathrm{Ce}$ & $\mathrm{Eu}$ & Th & Hf & $\mathrm{Ta}$ & $\mathrm{Sc}$ & $\mathrm{Sb}$ & $\mathrm{Se}$ \\
\hline 2000 & 40 & 0.5 & 0.1 & 0.2 & 0.1 & 0.5 & 2 & 0.1 & 0.05 & 100 & 1 & 0.05 & - & - & - & 0.005 & 0.1 & 0.018 & 5.0 & 0.1 & 0.05 \\
\hline 200 & 200 & 1.5 & 8.0 & 1.4 & 1.0 & 1.5 & 45 & 1.0 & 0.10 & 370 & 5 & 0.19 & 27 & 45 & 1.0 & 3.0 & 1.0 & 0.48 & 24.0 & 1.0 & 0.05 \\
\hline 50 & 100 & - & 15.0 & 0.9 & 1.0 & 20.0 & 100 & - & 0.007 & 500 & 15 & - & - & - & - & 7.0 & 1.0 & 0.70 & 2.5 & 0.2 & 0.05 \\
\hline 25 & 40 & 3.0 & 20.0 & 1.0 & 1.5 & 40.0 & 200 & 5.0 & 0.05 & 800 & 15 & 0.10 & 60 & 100 & 1.5 & 18.0 & 1.0 & 3.50 & 3.0 & 0.3 & 0.05 \\
\hline 100 & 130 & 10.0 & 20.0 & 2.0 & 2.1 & 60.0 & 200 & 12.0 & 0.10 & 500 & 100 & 0.3 & 40 & 50 & 1.0 & 11.0 & 6.0 & 3.50 & 10.0 & 0.5 & 0.61 \\
\hline 78 & 80 & 5.6 & - & 9.0 & - & - & - & - & - & - & 110 & $<5.0$ & - & - & - & - & - & - & - & - & - \\
\hline 95 & 80 & 5.8 & - & 15.0 & - & - & - & - & - & - & 168 & $<5.0$ & - & - & - & - & - & - & - & - & - \\
\hline 96 & 129 & - & - & 2.6 & - & - & - & - & - & - & - & - & - & - & - & - & - & - & - & - & - \\
\hline 71 & 89 & - & - & - & - & - & - & - & - & - & - & - & - & - & - & - & - & - & - & - & - \\
\hline 114 & 133 & - & - & - & - & - & - & - & - & - & - & - & - & - & - & - & - & - & - & - & - \\
\hline
\end{tabular}

Unit 10 is represented by compact brecciated aleurite-pelitic and fine-aleuritic muds (mudstones, siltstones) (37.97-48.16\% occurring in the $<0.01 \mathrm{~mm}$ fraction) with a mineral composition nearly the same as that of Unit 9. Chemical composition is much the same also, except for Sample 51-3, 26-28 cm, where $\mathrm{C}_{\text {org }}$ is $2.71 \%$.

Unit 11 is represented by laminated compact terrigenous clay aleurites (siltstones) (the $<0.01 \mathrm{~mm}$ fraction makes up $31.22-38.16 \%$ ) with rather low $\mathrm{CaCO}_{3}$ contents (1.00-6.50\%). Illite and chlorite are abundant. The clastic fraction is composed of feldspars and quartz. The micro-elements $(\mathrm{Ni}, \mathrm{Co}, \mathrm{Cr}, \mathrm{V}, \mathrm{Rb}, \mathrm{Cs})$ as well as Fe contents are high (Table 15). A bed at 52$2,62-63 \mathrm{~cm}$ consists, evidently, of ferruginous carbonates containing $20.70 \% \mathrm{Fe}$ and $0.35 \% \mathrm{Mn}$.

In general, the geochemical characteristics of lower Pleistocene and Neogene deposits at Site 381 are as follows:

1) Based on average contents (Table 16) both lower Pleistocene and upper Pliocene deposits consist of alternating low-calcareous and calcareous units. Noncalcareous deposits constitute a considerable part of the Miocene sediments (Units 9, 10,11).

Pliocene sediments (Units 5-7) are enriched in organic matter and amorphous silica (diatomaceous oozes). Unit 5 , besides having high $\mathrm{Cr}, \mathrm{V}, \mathrm{Rb}$, and $\mathrm{F}$ contents, contains the maximum Mn concentration for this site, due to diagenetic formation of carbonates of the manganosiderite-type.

Unit 8 (sandstones and breccias) is distinguished by rather low $\mathrm{Fe}, \mathrm{Mn}$, and $\mathrm{Ti}$ contents. The increased contents of $\mathrm{Ti}, \mathrm{Ni}, \mathrm{Cr}, \mathrm{Rb}, \mathrm{Cs}, \mathrm{Pb}$, and $\mathrm{Sn}$, as well as of $\mathrm{Li}$ (Unit 9), as well as contents of $\mathrm{Ni}, \mathrm{Cr}$, and $\mathrm{Sn}$ equal to $\mathrm{K}_{\mathrm{b}}$ are characteristic of the non-calcareous Miocene deposits composed of compact clays (black shales) (Units 9 and 11) and brecciated sediments (Unit 10). Due to ferruginous carbonates, the $\mathrm{Fe}$ content is markedly increased in a single sample of Unit 11.

2) The ratios of average element contents in these units to their clarkes in sedimentary rocks are variable for most elements (higher, equal, or lower than Ko; Table 16), except for the Sn contents which are 5-10 times lower than Ko.

Average element contents higher than Ko (see Table 9) approach their clarkes in basic rocks $\left(K_{b}\right)$ in Units 9 , 10,11 .

The ratios of average element contents to Ko suggest that the lower Pleistocene and Pliocene sediments formed mainly by deposition of weathered products of intermediate and acid rocks; erosional products of basic rocks were important in deposition of the rest of the sediment section (mainly Miocene).

\section{GEOCHEMISTRY OF SOME MACROCOMPONENTS \\ AND MINOR ELEMENTS (CARBONATES, SILICA, AND Corg)}

\section{Carbonates}

Up to $75 \%$ of $\mathrm{CaCO}_{3}$ is found in the Recent deepwater Black Sea sediments. Maximum contents are associated with the halistatic areas (Arkhangelsky and Strakhov, 1938; Shimkus and Trimonis, 1974, p. 266). In these areas almost $100 \%$ of the $\mathrm{CaCO}_{3}$ is made of coccoliths. Whereas the main basin is represented by coccolith calcite, the carbonate content is lower (10$30 \%$ ) along the periphery of the deep-water basin and in the center under the branches of the cyclonic currents (these areas include the drill sites). Clastic calcite, dolomite, and aragonite as admixtures are present here in small amounts (Trimonis, 1974). According to the data obtained by Müller and Stoffers (1974), regions of nannofossil oozes rich in organic matter contain needle-shaped ("rice grains") aragonite crystals of inorganic origin (Degens, 1971).

The late Cenozoic sediments at the sites drilled are characterized by different carbonate content and, particularly significant, different carbonate mineralogies.

Site 379: In most of the Quaternary section, $\mathrm{CaCO}_{3}$ contents do not exceed $30 \%$. Beds with higher $\mathrm{CaCO}_{3}$ 


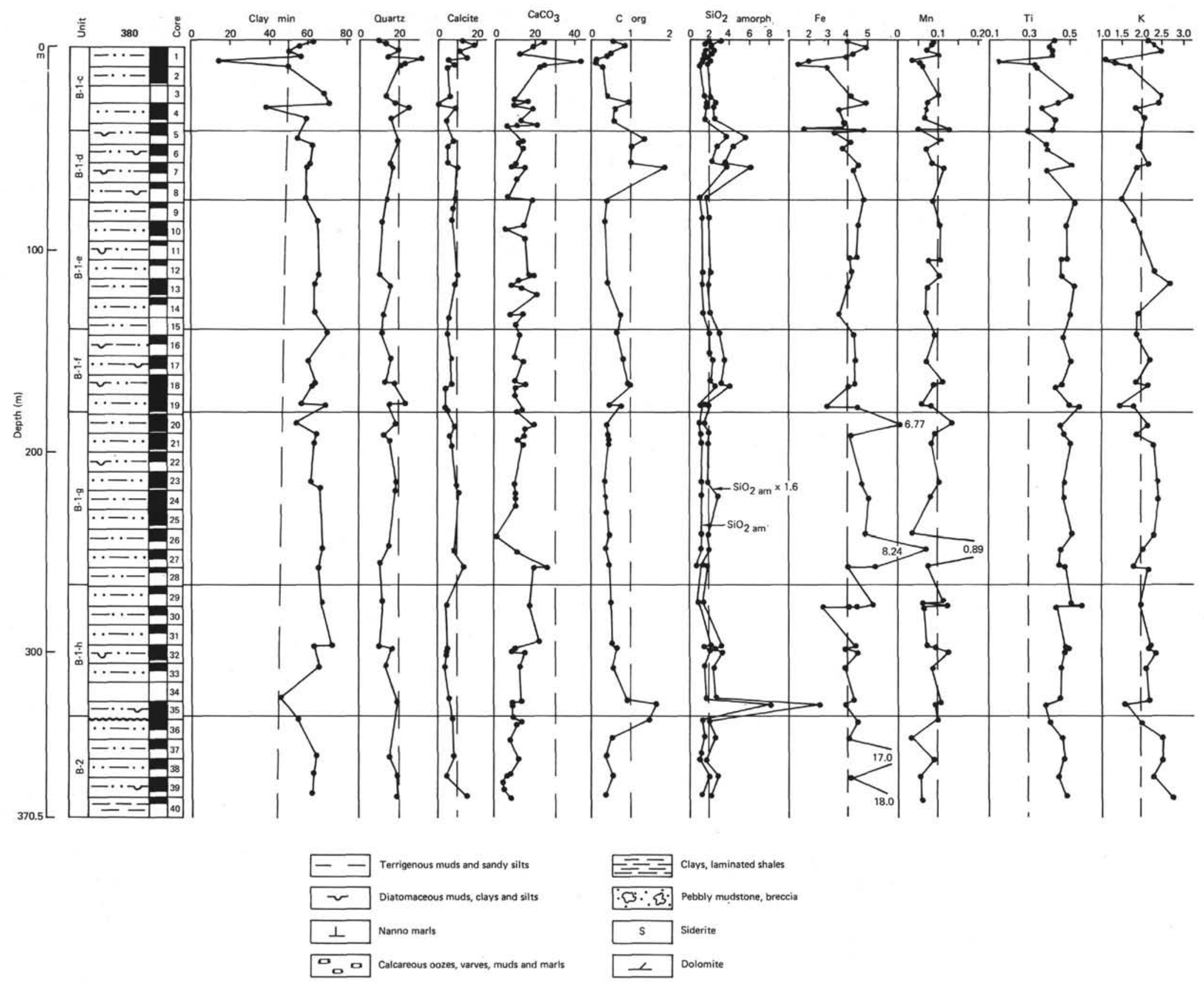

Figure 9. Lithologic composition of Site 380 sediments and distribution of main minerals and chemical macrocomponents. 

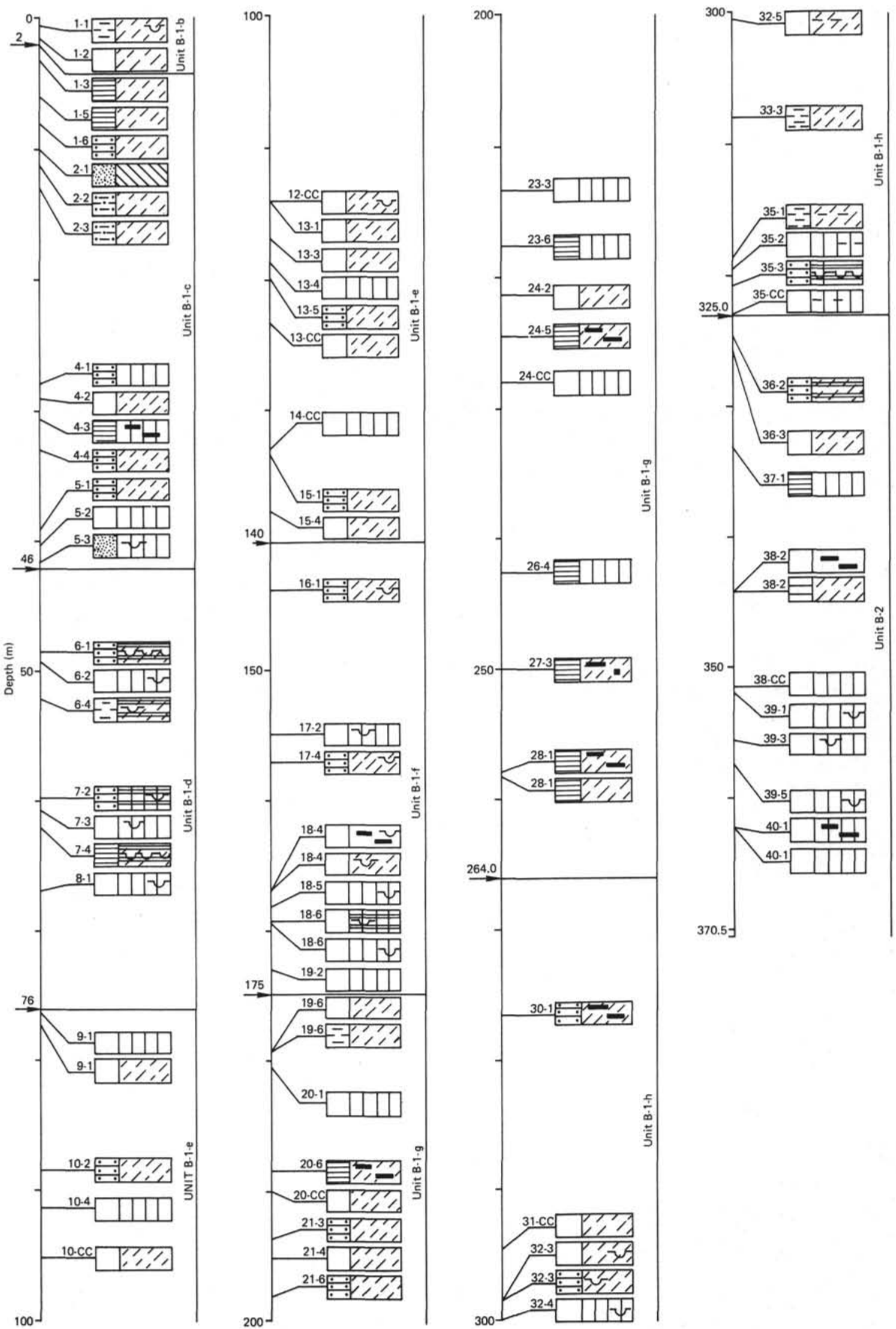

Figure 10. Composition of Site 380 sediments samples. 
TABLE 10

Chemical Composition of Bottom Sediments, Hole 380

\begin{tabular}{|c|c|c|c|c|c|c|c|c|c|c|c|c|c|c|c|c|c|c|c|c|c|c|c|c|c|c|c|}
\hline \multirow{2}{*}{$\begin{array}{l}\text { Depth } \\
\text { (m) }\end{array}$} & \multirow{2}{*}{$\begin{array}{c}\text { Sample } \\
\text { (Interval in cm) }\end{array}$} & \multicolumn{9}{|c|}{ Content (\%) } & \multicolumn{17}{|c|}{ Content $\left(10^{-4} \%\right)$} \\
\hline & & $\mathrm{CaCO}_{3}$ & $\mathrm{C}_{\text {org }}$ & $\mathrm{SiO}_{2}$ & $\mathrm{Fe}$ & $\mathrm{Mn}$ & $\mathrm{Ti}$ & P & K & $\mathrm{Na}$ & $\mathrm{Cu}$ & $\mathrm{Zn}$ & $\mathrm{Ni}$ & $\mathrm{Co}$ & $\mathrm{Cr}$ & v & $\mathrm{Ca}$ & $\mathrm{Li}$ & $\mathrm{Rb}$ & Cs & $\mathrm{Pb}$ & $\mathrm{Sn}$ & $\mathrm{Se}$ & Mo & w & $\mathrm{F}$ & B \\
\hline 0.60 & $1-1, \quad 60-68$ & 21.25 & 0.56 & 1.74 & 4.03 & 0.09 & 0.43 & 0.05 & 2.13 & 1.30 & 35 & 94 & 60 & 14 & 100 & 120 & $<6$ & 14 & 50 & 5.0 & 25 & 1.2 & 0.2 & 1.6 & 13 & - & - \\
\hline 1.52 & $1-2, \quad 2-4$ & 21.00 & - & - & - & - & - & - & $\therefore$ & - & - & - & $=$ & - & - & - & - & - & - & - & - & - & - & - & - & - & - \\
\hline 3.10 & $1-3, \quad 10-20$ & 16.87 & 0.91 & 1.20 & 4.97 & 0.08 & 0.41 & 0.06 & 2.25 & 1.11 & 61 & 101 & 76 & - & 114 & - & $<6$ & 15 & 50 & 6.0 & 16 & 1.4 & 0.4 & 2.1 & 23 & - & - \\
\hline 6.10 & $1-5, \quad 10-20$ & 11.48 & 0.55 & 1.27 & 4.21 & 0.07 & 0.41 & 0.06 & 2.45 & 0.98 & 41 & 97 & 70 & - & 123 & - & $<6$ & 12 & 40 & 5.0 & 12 & 1.6 & - & - & - & - & - \\
\hline 8.21 & $1-6, \quad 71-81$ & 24.22 & 0.45 & 1.20 & 3.85 & 0.10 & 0.41 & 0.05 & 1.91 & 0.79 & 29 & 87 & 68 & - & 108 & - & $<6$ & 12 & 35 & 4.5 & 11 & 1.2 & 0.2 & - & - & - & - \\
\hline 10.12 & $2-1, \quad 62-67$ & 41.48 & 0.18 & 0.97 & 1.88 & 0.03 & 0.15 & 0.03 & 1.09 & 0.82 & 13 & 37 & 30 & (3) & 28 & - & $<6$ & 10 & 30 & 3.5 & 6 & 1.0 & - & - & - & - & - \\
\hline 11.40 & $2-2,40-52$ & 22.11 & 0.20 & 1.15 & 2.33 & 0.05 & 0.34 & 0.04 & 1.35 & 1.00 & 13 & 43 & 42 & - & 63 & - & $<6$ & 12 & 40 & 4.5 & 13 & 1.3 & 0.2 & 1.6 & 13 & - & - \\
\hline 13.07 & $2-3,57-70$ & 20.78 & 0.36 & 0.92 & 2.87 & 0.06 & 0.34 & 0.04 & 1.68 & 0.91 & 29 & 66 & 56 & - & 89 & - & $<6$ & 20 & 50 & 4.5 & 18 & 2.0 & - & - & - & - & - \\
\hline 27.69 & $4-1, \quad 19-28$ & 9.06 & 0.43 & 1.17 & 4.21 & 0.10 & 0.52 & 0.07 & 2.51 & 0.96 & 33 & 105 & 76 & 33 & 132 & 180 & $<6$ & - & - & - & - & - & 0.4 & 1.7 & 30 & - & - \\
\hline 29.10 & $4-2, \quad 10-20$ & 15.00 & - & - & - & - & - & - & - & - & - & - & - & - & - & - & - & - & - & - & $\overline{-}$ & - & - & $=$ & $\bar{x}$ & - & - \\
\hline 30.68 & $4-3, \quad 18-31$ & 4.42 & 0.99 & 1.41 & 5.02 & 0.07 & 0.45 & 0.06 & 2.44 & 0.90 & 58 & 117 & 87 & - & 124 & - & $<6$ & 20 & 60 & 5.2 & 25 & 2.5 & 0.9 & 2.6 & 25 & - & - \\
\hline 333.20 & $4-4,120-133$ & 18.31 & 0.64 & 1.32 & 3.58 & 0.07 & 0.37 & 0.06 & 1.86 & 0.82 & 41 & 81 & 76 & - & 104 & - & $<6$ & 16 & 45 & 4.8 & 15 & 1.5 & 0.5 & 2.6 & 20 & - & - \\
\hline 38.82 & $5-1,82-95$ & 11.18 & 0.60 & 1.38 & 3.85 & 0.07 & 0.44 & 0.06 & 2.08 & 0.93 & 34 & 92 & 76 & - & 114 & - & $<6$ & 20 & 50 & 5.0 & 14 & 1.8 & - & - & - & - & - \\
\hline 40.24 & $5-2, \quad 74-92$ & 10.00 & - & - & - & - & - & - & - & - & - & - & - & - & - & - & - & - & - & - & - & - & - & - & - & - & - \\
\hline Average & & - & 0.53 & 1.25 & 3.71 & 0.07 & 0.39 & 0.05 & 1.98 & 0.96 & 35 & 84 & 65 & 17 & 100 & 150 & $<6$ & 15 & 45 & 5.0 & 15 & 1.6 & 0.4 & 2.0 & 21 & - & - \\
\hline 41.49 & $5-3,49-62$ & 5.00 & - & - & - & - & - & - & - & - & - & - & - & - & - & - & - & - & - & - & - & - & - & - & - & - & - \\
\hline 42.99 & $5-4,49-51$ & - & - & - & 3.87 & 0.12 & 0.43 & - & - & - & - & - & - & - & - & - & - & - & - & - & - & - & - & - & - & - & - \\
\hline 43.12 & $5-4,62-64$ & - & - & - & 1.88 & 0.05 & 0.30 & - & - & - & - & - & - & - & - & - & - & - & - & - & - & - & - & - & - & - & - \\
\hline 43.17 & $5-4, \quad 67-69$ & - & - & - & 4.56 & 0.08 & 0.43 & - & - & - & - & - & - & - & - & - & - & - & - & - & - & - & - & - & - & - & - \\
\hline 43.43 & $5-4, \quad 93-95$ & - & - & - & 3.24 & 0.06 & 0.43 & - & - & - & - & - & - & (13) & - & - & - & - & - & - & - & - & - & - & - & - & - \\
\hline 48.43 & $6-1,93-105$ & 12.64 & 1.40 & 3.50 & 4.12 & 0.10 & 0.39 & 0.07 & - & - & - & - & $\overline{-}$ & $\overline{-}$ & - & - & - & - & - & - & - & - & 0.9 & 4.9 & 26 & - & - \\
\hline 49.35 & $6-2, \quad 35-45$ & 10.00 & - & - & - & - & - & - & - & - & - & - & 52 & 12 & 92 & 110 & - & - & - & - & - & - & - & - & - & 610 & - \\
\hline 52.12 & $6-4, \quad 12-33$ & 13.14 & 1.09 & 2.66 & 3.81 & 0.07 & 0.40 & 0.05 & 1.94 & 1.07 & 31 & 86 & 73 & - & 103 & - & $<6$ & 20 & 60 & 5.2 & 14 & 2.5 & 0.7 & 2.2 & 13 & - & - \\
\hline 59.72 & $7-2,122-137$ & 9.58 & 1.08 & 2.11 & 4.54 & 0.09 & 0.52 & 0.05 & 2.12 & 0.76 & 36 & 97 & 72 & - & 123 & - & $<6$ & 25 & 70 & 6.5 & 18 & 2.6 & - & - & - & - & - \\
\hline 60.78 & $7-3, \quad 78-86$ & 7.00 & - & - & - & - & - & - & - & - & - & - & $\bar{x}$ & - & - & - & - & - & - & - & - & - & - & - & - & - & - \\
\hline 61.92 & $7-4, \quad 42-54$ & 15.04 & 1.94 & 3.78 & 4.30 & 0.11 & 0.40 & 0.07 & 1.97 & 1.10 & 46 & 87 & 76 & - & 110 & - & $<6$ & 16 & 60 & 5.0 & 14 & 2.5 & 1.0 & 3.1 & 23 & - & - \\
\hline 66.83 & $8-1, \quad 33-48$ & 10.00 & - & - & - & - & - & - & - & - & - & - & 72 & 19 & 85 & 120 & - & - & - & - & - & - & - & - & - & - & - \\
\hline Average & & & 1.38 & 3.01 & 3.79 & 0.08 & 0.41 & 0.06 & 2.00 & 0.98 & 38 & 90 & 69 & 16 & 103 & 115 & $<6$ & 20 & 63 & 5.6 & 15 & 2.5 & 0.9 & 3.4 & 21 & 610 & - \\
\hline 76.41 & $9-1, \quad 41-43$ & 5.50 & - & - & - & - & - & - & - & - & - & - & - & - & - & - & - & 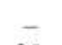 & - & - & - & - & - & - & - & - & - \\
\hline 77.15 & $9-1,115-130$ & 17.44 & 0.43 & 1.02 & 4.84 & 0.09 & 0.53 & 0.06 & 1.58 & 1.11 & 37 & 89 & 83 & - & 111 & - & $<5$ & 25 & 70 & 5.2 & 14 & 2.0 & 0.4 & 1.6 & 16 & - & - \\
\hline 88.25 & $10-2,125-138$ & 14.17 & 0.41 & 1.11 & 4.57 & 0.11 & 0.50 & 0.06 & 1.89 & 1.01 & 36 & 89 & 82 & $\overline{0}$ & 115 & - & $<5$ & 20 & 70 & 5.4 & 11 & 1.6 & 0.3 & 1.6 & 14 & - & - \\
\hline 91.06 & $10-4,106-132$ & 5.00 & - & - & - & - & - & - & - & - & - & - & 60 & 23 & 48 & 140 & $<5$ & - & - & - & - & - & - & - & - & 410 & - \\
\hline 95.00 & $10, \mathrm{CC}$ & 14.00 & - & - & - & - & - & - & - & - & - & - & - & - & - & - & - & - & - & - & - & - & - & - & - & - & - \\
\hline 105.80 & $12-1,130-131$ & - & - & - & 4.49 & 0.11 & 0.50 & - & - & - & - & - & - & (13) & (90) & - & - & - & - & - & - & - & - & - & - & - & - \\
\hline 105.86 & $12-1,136-137$ & - & - & - & 4.21 & 0.08 & 0.47 & - & - & - & - & - & - & - & - & - & - & - & - & - & - & - & - & - & - & - & - \\
\hline 114.00 & 12. CC & 16.00 & - & - & - & - & - & - & - & - & - & - & - & - & - & - & - & - & - & - & - & - & - & - & - & - & - \\
\hline 114.00 & $13-1, \quad 0-14$ & 18.94 & 0.46 & 1.25 & 4.30 & 0.11 & 0.47 & 0.05 & 2.32 & 1.04 & 42 & 93 & 84 & - & 118 & - & $<5$ & 20 & 60 & 5.0 & 15 & 2.0 & - & 1.6 & 13 & - & - \\
\hline & $13-3, \quad 0-13$ & & - & - & - & - & - & - & - & - & - & - & - & - & - & - & - & - & - & - & - & - & - & - & - & - & - \\
\hline 118.71 & $13-4,21-34$ & 7.50 & - & - & - & - & - & - & - & - & - & - & - & - & - & $\begin{array}{l}- \\
-\end{array}$ & - & - & - & - & - & - & - & - & - & - & - \\
\hline 120.02 & $13-5, \quad 2-14$ & 12.41 & 0.47 & 1.17 & 4.03 & 0.08 & 0.53 & 0.06 & 2.76 & 1.48 & 43 & 102 & 82 & 20 & 121 & 140 & $<5$ & 25 & 70 & 6.0 & 14 & 2.0 & 0.3 & 2.6 & 16 & 450 & - \\
\hline 123.50 & $13, \mathrm{CC}$ & 21.00 & - & - & - & - & - & - & - & - & - & - & - & - & - & - & - & - & - & - & - & - & - & - & - & - & - \\
\hline 133.00 & $14, \mathrm{CC}$ & 7.00 & - & - & - & - & - & - & - & - & - & - & - & - & - & - & - & - & - & - & - & - & - & - & - & - & - \\
\hline .30 & $15-1, \quad 30-45$ & 13.74 & 0.74 & 1.30 & 3.72 & 0.08 & 0.51 & 0.05 & 1.91 & 1.33 & 34 & 80 & 67 & - & 112 & - & $<5$ & 35 & 90 & 6.0 & 12 & 2.3 & 0.3 & - & - & - & - \\
\hline 137.80 & $15-4, \quad 30-45$ & 10.50 & - & - & - & - & - & - & - & - & - & - & - & - & - & - & - & - & - & - & - & - & - & - & - & - & - \\
\hline Average & - & 12.44 & 0.50 & 1.17 & 4.31 & 0.09 & 0.50 & 0.06 & 2.09 & 1.19 & 38 & 91 & 76 & 20 & 102 & 140 & $<5$ & 25 & 72 & 5.5 & 13 & 2.0 & 0.3 & 1.8 & 15 & 470 & - \\
\hline & & & 0.70 & 1.85 & $\cdots$ & 0.10 & 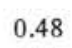 & 0.00 & 1.91 & 1. & 3 & 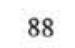 & 1 & - & 11 & - & $<$ & 30 & 0 & 6.0 & 16 & 2.2 & 0.4 & 4.0 & 20 & - & - \\
\hline 154.80 & $17-2,130-148$ & 8. & - & - & - & - & - & - & - & - & - & - & 70 & 23 & 78 & 110 & - & - & - & - & - & - & - & - & - & 560 & 128 \\
\hline 156.95 & $17-4,45-60$ & & 0.83 & 2.19 & 4.48 & 0.08 & 0.52 & 0.05 & 2.24 & 1.45 & 35 & 90 & 72 & - & 114 & - & $<5$ & 40 & 80 & 6.0 & 20 & 2.2 & - & - & - & - & - \\
\hline 167.06 & $18-4,106-125$ & 13.39 & 0.93 & 2.01 & 4.48 & 0.12 & 0.47 & 0.07 & 1.96 & 1.01 & 42 & 92 & 78 & - & 117 & - & $<5$ & 50 & 90 & 7.0 & 22 & 2.4 & - & - & - & - & - \\
\hline 168.06 & $18-5,56-68$ & 9.50 & - & - & - & - & - & - & - & - & - & - & - & - & - & - & - & - & - & - & - & - & - & - & - & - & - \\
\hline 169.36 & $18-6,36-38$ & 10.00 & 1.06 & 2.55 & 4.12 & 0.10 & 0.45 & 0.06 & - & - & - & - & - & - & - & - & - & - & - & - & - & - & - & - & - & - & - \\
\hline
\end{tabular}


$169.41 \quad 18-6,41-43$

$\begin{array}{ll}170.30 & 18-6,130-148 \\ 173.00 & 19-2,50-53\end{array}$

Average

9.00

9.00

$\begin{array}{lll}10.49 & 0.88 & 2.15\end{array}$

Unit B-1-g

$179.08 \quad 19-6,58-70$

$179.36 \quad 19-6,86-100$

$180.52 \quad 20-6,2-12$

$188.40 \quad 20-6,40-50$

190.0020 , CC

$193.56 \quad 21-3,56-69$

$195.05 \quad 21-4,55-68$

$197.98 \quad 21-6,48-61$

$213.29 \quad 23-3,129-13$

217.50 23-6, 100-110

218.60 23, CC

$221.30 \quad 24-2,130-140$

$224.50 \quad 24-5,0-8$

$228.00 \quad 24, \mathrm{CC}$

$242.55 \quad 26-4,55-60$

$257.74 \quad 28-1,124-136$

$257.84 \quad 28-1,134-150$

Average

12.38

$\begin{array}{llllllllll}2.38 & 0.47 & 0.98 & 3.05 & 0.07 & 0.52 & 0.05 & 1.56 & 1.19 & 20\end{array}$

10.00

18.50

$\begin{array}{lllllllll}0.40 & 0.90 & 6.77 & 0.15 & 0.47 & 0.09 & 2,24 & 1.16 & 38\end{array}$

13.78

$-$

$\begin{array}{llllllllllll}9.33 & 0.38 & 1.24 & 4.75 & 0.11 & 0.48 & 0.09 & 2.47 & 1.16 & 45 & 103 & 98\end{array}$

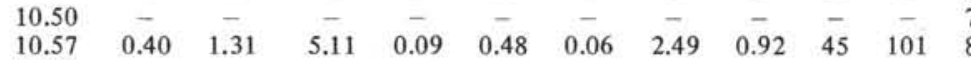

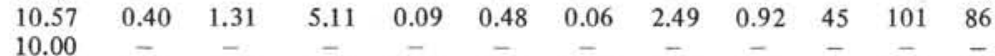

$\begin{array}{llllllllllll}0.85 & 0.46 & 1.24 & 4.98 & 0.04 & 0.52 & 0.06 & 2.41 & 1.45 & 57 & 118 & 98\end{array}$

$\begin{array}{llllllllllll}10.24 & 0.40 & 1.26 & 8.24 & 0.98 & 0.47 & 0.06 & 2.08 & 1.01 & 45 & 95 & 71\end{array}$

$\begin{array}{rrrrrrrrrrrr}24.92 & 0.47 & 0.77 & 5.56 & 0.11 & 0.46 & 0.07 & 1.83 & 0.89 & 38 & 85 & 78 \\ 19.55 & 0.47 & 1.12 & 4.12 & 0.08 & 0.49 & 0.06 & 2.19 & 1.04 & 43 & 101 & 98\end{array}$

$\begin{array}{lll}12.44 & 0.47 & 1.13\end{array}$

$\begin{array}{lllllll}4.87 & 0.16 & 0.49 & 0.07 & 2.13 & 1.11 & 40\end{array}$

Unit B-1-h

$276,44 \quad 30-1,94-110$

$277.64 \quad 30-2,64-67$

$\begin{array}{ll}277.64 & 30-2,64-67 \\ 277.70 & 30-2,70-73\end{array}$

$277.79 \quad 30-2,79-82$

$277.91 \quad 30-2,91-94$

$294.5031, \mathrm{CC}$

$298.20 \quad 32-3,70-80$

$298.30 \quad 32-3,80-90$

$299.73 \quad 32-4,73-83$

$\begin{array}{ll}300.51 & 32-5,1-10 \\ 30.95 & 33-3,95-110\end{array}$

$\begin{array}{ll}307.95 & 33-3,95-110 \\ 318.61 & 35-1,111-123\end{array}$

$\begin{array}{ll}318.61 & 35-1,111-123 \\ 35-2,54-64\end{array}$

$320.69 \quad 35-3,19-42$

$323.00 \quad 35, \mathrm{CC}$

Average

$324.78 \quad 36-2,28-38$

$326.58 \quad 36-3,58-68$

$333.23 \quad 37-1,73-87$

$333.23 \quad 38-2,30-31$

$344.23 \quad 38-2,73-8$

$351.50 \quad 38, \mathrm{CC}$

$351.98 \quad 39-1,48-62$

$355.54 \quad 39-3,104-115$

$357.50 \quad 39-5,0-12$

$357.50 \quad 40-1,76-78$

$362.20 \quad 40-1,120-135$

Average

$\begin{array}{lll}16.74 & 0.47 & 0.92\end{array}$

$\begin{array}{lllllll}5.38 & 0.12 & 0.53 & 0.07 & 1.99 & 1.13 & 36\end{array}$

$94 \quad 80$

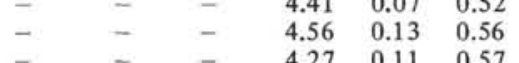

$\begin{array}{llllllllll}10.04 & 0.56 & \overline{2.12} & \overline{4.39} & 0.08 & 0.49 & 0.06 & \overline{2.26} & \overline{1.19} & 43\end{array}$

$\begin{array}{llllllllll}0.54 & 0.59 & 1.82 & 3.94 & 0.10 & 0.50 & 0.06 & 2.22 & 1.20 & 44\end{array}$

$\begin{array}{llllllllll}14.33 & 0.65 & 2.26 & 4.66 & 0.13 & 0.48 & 0.06 & 2.41 & 1.48 & 40\end{array}$

$\begin{array}{llllllllll}1.07 & 0.57 & 1.70 & 3.94 & 0.09 & 0.47 & 0.06 & 2.14 & 1.47 & 31 \\ 13.06 & 0.91 & 1.93 & 4.39 & 0.11 & 0.47 & 0.05 & 2.19 & 1.48 & 36\end{array}$

$\begin{array}{rrrrrrrrrrr}1.06 & 0.91 & 1.93 & 4.39 & 0.11 & 0.47 & 0.05 & 2.19 & 1.48 & 36\end{array}$

$\begin{array}{llllllllll}8.86 & 1.64 & \overline{8.22} & 3 . \overline{4} & 0 . \overline{10} & \overline{0.39} & \overline{0.05} & \overline{1.66} & \overline{1.66} & \overline{50}\end{array}$

8.50

$\begin{array}{lllllllll}0.77 & 2.71 & 4.24 & 0.10 & 0.49 & 0.06 & 2.12 & 1.37 & 40\end{array}$

$\begin{array}{llllllllll}12.34 & 1.42 & 1.40 & 4.62 & 0.10 & 0.42 & 0.05 & 1.95 & 1.19 & 48\end{array}$

$\begin{array}{rrrrrrrrrr}7.62 & 0.50 & 1.77 & 4.17 & 0.04 & 0.48 & 0.05 & 2.49 & 1.29 & 49\end{array}$

\begin{tabular}{llllllllll}
\hline & $\overline{1}$ & $\overline{1}$ & 16.20 & 0.24 & 0.35 & $\overline{1}$ & 1.58 & 1.08 & 31
\end{tabular}

$\begin{array}{llllllllll}7.50 & & 1.22 & 4.21 & 0.09 & 0.49 & 0.04 & 2.54 & 1.48 & 44\end{array}$

$\begin{array}{llllllllll}5.81 & 0.53 & 1.95 & 4.35 & \overline{0.06} & 0.47 & \overline{0.05} & \overline{2.42} & \overline{1.42} & \overline{48}\end{array}$

4.00

4.50

$\begin{array}{lllrllllll}- & - & - & 16.80 & 0.28 & 0.35 & - & 1.54 & 0.93 & 24 \\ 7.69 & 0.36 & 1.46 & 4.89 & 0.06 & 0.50 & 0.06 & 2.90 & 1.31 & 43\end{array}$

$\begin{array}{lllllllll}0.63 & 1.56 & 7.89 & 0.12 & 0.44 & 0.05 & 2.20 & 1.24 & 41\end{array}$
$102-<50$

$\begin{array}{lllllll}60 & 5.2 & 20 & 2.6 & 1.0 & 3.2 & 20\end{array}$

$<38$

$\begin{array}{lllllllll}78 & 6.0 & 20 & 2.4 & 0.7 & 3.6 & 20 & 560 & 128\end{array}$

$\begin{array}{lll}-\quad 2.77 \quad 0.07 & 0.45\end{array}$
9178

$16100-130=2$

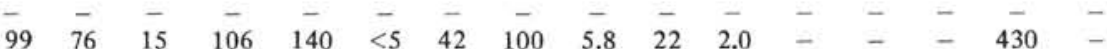

$\begin{array}{rrrrrrrrrrrrrrrr}90 & 60 & - & 116 & - & <4 & 40 & 90 & 5.8 & 18 & 1.8 & 0.4 & 0.8 & 25 & - & -\end{array}$

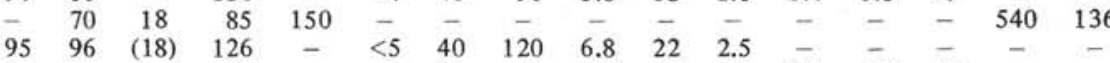

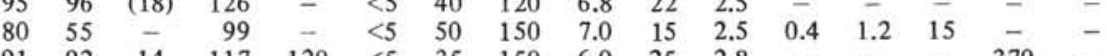

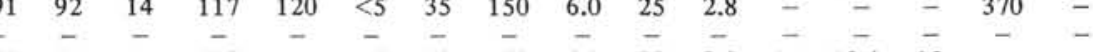

$\begin{array}{llllllllllllllll}87 & 74 & 16 & 106 & 135 & <5 & 39 & 109 & 5.9 & 21 & 2.3 & 0.7 & 3.8 & 21 & 446 & 136\end{array}$

$\begin{array}{llllllllllllllll}88 & 80 & 22 & 72 & 140 & <4 & 40 & 100 & 6.6 & 30 & 2.8 & 1.4 & 4.8 & 16 & 410 & -\end{array}$

9176

$\begin{array}{ll}74 & 53 \\ 87 & 54\end{array}$

\begin{tabular}{l}
87 \\
$-\quad 70$ \\
\hline
\end{tabular}

$\overline{73} \quad \overline{31}$

$\begin{array}{lll}73 & 31 & <4 \\ 97 & 61 & 15\end{array}$

$\begin{array}{llll}140 & \overline{170} & <4 & 35\end{array}$

$98 \quad 160<5 \quad 35$

$89 \quad 76$ $\begin{array}{rrrrr}86 & 72 & 16 & 85 & 180\end{array}$ 


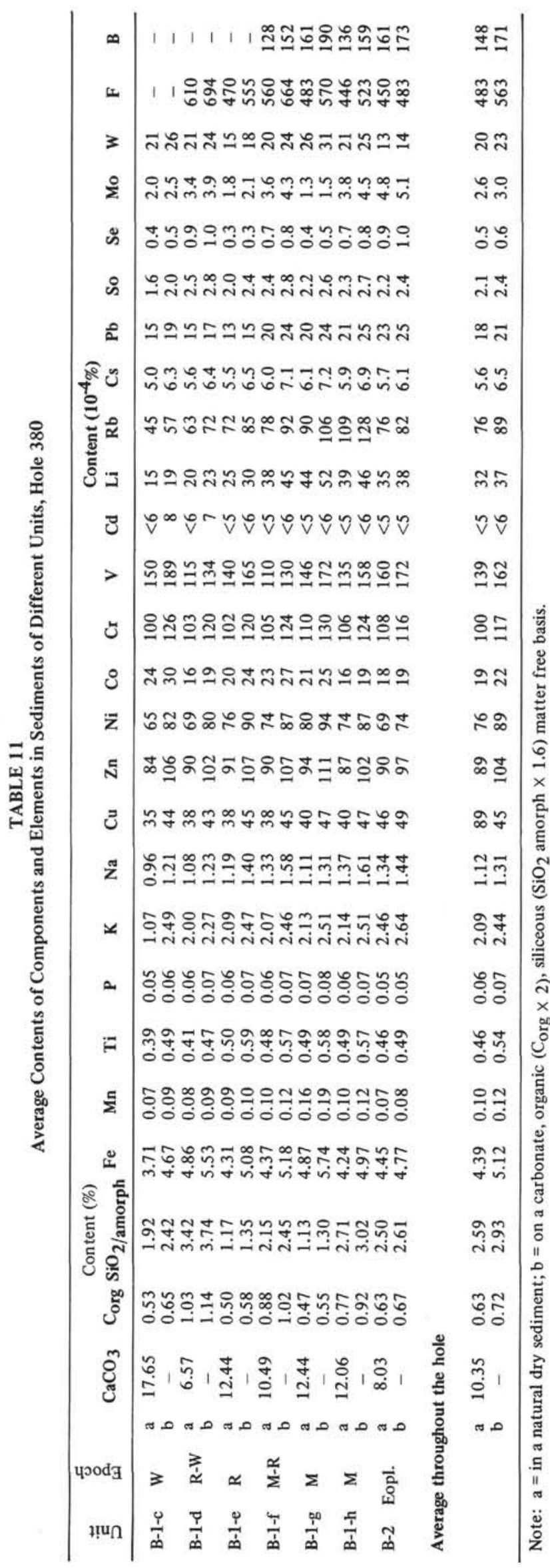

content were found only in the lower Interglacial units (Units 7 and 9, M-R and G-M). Contents higher than $50 \%$ were observed in some cases (Table 17).

The distribution of $\mathrm{CaCO}_{3}$ in the Glacial units is characterized by rapid fluctuations over narrow ranges. In the interglacial and interstadial series these ranges are much wider, and minimum and maximum values (7and 9) are often found here.

According to the data obtained by Trimonis et al. (this volume), the carbonates in the whole Quaternary sediments of Site 379 are mostly calcite. A small admixture (up to $3-4 \%$ of the crystalline phase) of dolomite is present, only rarely being a dominant phase (Sample 8-1, 9-11 cm, for example).

Dolomite, where present, is usually accompanied by up to $40 \%$ calcite; dolomite was not found at calcite concentrations greater than $50 \%$. In some beds of Interglacial Unit 5 (R-W) enriched in Corg, from 4.6 to $13.2 \%$ of aragonite $(11-5,24-25 \mathrm{~cm}, 11-5,37-38 \mathrm{~cm}, 11-$ $5,53-55 \mathrm{~cm}$ ) was found. It should be noted that dolomite was not found in this unit and that the aragonite was represented by needle-shaped "rice grain" crystals, suggesting a chemogenic origin.

Thus, the carbonates from Site 379 beds consist of either calcite or calcite with a small admixture of dolomite, or of calcite and aragonite. Calcite and dolomite are ubiquitous, while aragonite is only found in sediments of the last Interglacial stage.

It should be noted that those beds with a high calcite content $(>50 \%)$ were few and were always associated with the Interglacial series $(29-5,107-112 \mathrm{~cm}$, Unit 7 [M-R]; 57-5, 130-132 cm, Unit 9, up to G-M). Microscopic examination shows that the calcite is authigenic. In those beds with a lower calcite content, the calcite is probably clastic, although in some cases chemogenic calcite was found as an admixture.

Site 380: This site is characterized by low carbonate contents (often lower than 15\%) in the Quaternary sediment section. Only in the upper unit (B-1-c) do values exceed $20 \%$; occasionally beds with values higher than $40 \%(2-1,62-67 \mathrm{~cm})$ were observed. $\mathrm{CaCO}_{3}$ content and its variations are almost the same in both the Glacial and Interglacial units.

The carbonate mineral composition at this site significantly differs from that of Site 379 . With calcite, dolomite is almost always quantitatively an important carbonate phase. Only Unit B-1-g (M) and deeper parts of the section are characterized by the absence of dolomite; there, calcite is the only carbonate present. There are beds where dolomite prevails over calcite and a few single beds are composed of dolomite exclusively. Site 380 differs from Site 379 in that sporadic siderite occurs in the lower part of the section, beginning from Unit B-1-g (M). It is usually found with calcite and dolomite and is of subordinate importance; it rarely prevails over dolomite. In the upper unit (B-1-C, W), beds were found where aragonite was observed in amounts similar to those of calcite and dolomite.

Hole 380A: In this hole, the quantitative distribution of carbonates is complex and the carbonate mineralogy is unusual. This is related to the presence of Pliocene sediments which were not encountered at Site 
TABLE 12

Total Chemical Composition (\%) of Black Sea Bottom Sediments

\begin{tabular}{|c|c|c|c|c|c|c|c|c|c|c|c|c|c|c|c|c|c|c|}
\hline Unit & $\begin{array}{c}\text { Sample } \\
\text { (Interval in } \mathrm{cm} \text { ) }\end{array}$ & $\mathrm{SiO}_{2}$ & $\mathrm{TiO}_{2}$ & $\mathrm{Al}_{2} \mathrm{O}_{3}$ & $\mathrm{FeO}$ & $\begin{array}{l}\mathrm{Fe}_{2} \mathrm{O}_{3} \\
\text { oxide }\end{array}$ & $\mathrm{MnO}$ & $\mathrm{MgO}$ & $\mathrm{CaO}$ & $\mathrm{P}_{2} \mathrm{O}_{5}$ & S & $\mathrm{F}$ & $\mathrm{H}_{2} \mathrm{O}$ & L.O.I. & $\mathrm{CO}_{2}$ & $\mathrm{~K}_{2} \mathrm{O}$ & $\mathrm{Na}_{2} \mathrm{O}$ & $\underset{\left(\text { by } \mathrm{CO}_{2}\right)}{\mathrm{CaCO}_{3}}$ \\
\hline \multicolumn{19}{|c|}{ Hole 379A } \\
\hline 3 & $6, \mathrm{CC}$ & 52.34 & 1.01 & 13.95 & 2.54 & 3.18 & 0.11 & 3.12 & 8.40 & 0.24 & 0.22 & 0.06 & 4.56 & 10.50 & 5.88 & 2.25 & 1.59 & 13.37 \\
\hline 4 & $11-4,135-143$ & 47.16 & 0.96 & 14.33 & 2.90 & 3.05 & 0.11 & 3.96 & 10.72 & 0.14 & 0.62 & 0.06 & 4.66 & 12.07 & 7.35 & 2.25 & 1.59 & 16.72 \\
\hline 6 & $17-1,25-35$ & 51.55 & 1.02 & 15.87 & 3.63 & 3.46 & 0.12 & 3.55 & 5.53 & 0.16 & 1.15 & 0.03 & 5.04 & 9.40 & 4.33 & 2.53 & 1.40 & 9.85 \\
\hline 7 & $19-5,107-112$ & 14.31 & 0.42 & 4.69 & 1.33 & 1.01 & 0.17 & 1.42 & 40.35 & 0.16 & 0.36 & 0.003 & 1.94 & 34.00 & 32.07 & 0.72 & 0.75 & 72.93 \\
\hline 8 & $43-3,140-150$ & 44.81 & 1.03 & 14.39 & 2.90 & 4.33 & 0.18 & 4.47 & 10.70 & 0.16 & 0.10 & 0.06 & 5.02 & 13.28 & 8.20 & 2.22 & 0.90 & 18.65 \\
\hline 9 & $50-5,12-24$ & 43.08 & 0.94 & 11.21 & 3.02 & 2.55 & 0.11 & 3.88 & 15.30 & 0.21 & 0.15 & 0.04 & 4.70 & 16.48 & 11.74 & 1.66 & 1.36 & 26.70 \\
\hline 9 & $59-5,5-13$ & 43.32 & 1.01 & 13.76 & 2.90 & 3.72 & 0.11 & 3.85 & 12.30 & 0.15 & 0.18 & 0.06 & 5.02 & 15.00 & 9.21 & 2.11 & 0.86 & 20.94 \\
\hline \multicolumn{19}{|c|}{ Hole 380} \\
\hline B-1-c & $1-1,60-68$ & 47.70 & 0.90 & 13.29 & 2.90 & 2.21 & 0.11 & 3.72 & 10.03 & 0.15 & 0.71 & 0.01 & 4.94 & 13.97 & 9.02 & 2.50 & 1.51 & 20.51 \\
\hline B-1-c & $2-2,40-52$ & 58.93 & 0.73 & 9.44 & 1.69 & 1.32 & 0.09 & 2.05 & 11.22 & 0.14 & 0.10 & 0.03 & 2.00 & 11.20 & 9.16 & 1.79 & 1.46 & 20.83 \\
\hline$B-1-d$ & $6-4,12-33$ & 52.83 & 0.99 & 12.86 & 2.30 & 3.02 & 0.07 & 3.22 & 7.58 & 0.14 & 1.08 & 0.03 & 3.64 & 10.98 & 7.30 & 2.34 & 1.49 & 16.60 \\
\hline B-1-e & $13-1,0-14$ & 45.75 & 0.98 & 15.72 & 3.02 & 3.01 & 0.30 & 4.37 & 8.48 & 0.12 & 0.06 & 0.02 & 4.42 & 13.60 & 9.16 & 2.92 & 1.48 & 20.83 \\
\hline$B-1-f$ & $18-6,130-148$ & 51.68 & 1.01 & 15.06 & 3.02 & 3.07 & 0.11 & 3.45 & 5.98 & 0.10 & 1.30 & 0.16 & 4.56 & 10.22 & 5.50 & 2.79 & 1.30 & 12.51 \\
\hline B-1-g & $20-6,40-50$ & 46.97 & 1.01 & 15.28 & 5.92 & 2.71 & 0.16 & 2.94 & 7.00 & 0.17 & 0.08 & 0.07 & 4.24 & 13.09 & 8.96 & 2.85 & 1.30 & 20.38 \\
\hline$B-1-g$ & $26-4,55-65$ & 56.71 & 1.09 & 18.46 & 2.75 & 4.32 & 0.10 & 2.56 & 1.19 & 0.11 & 0.26 & 0.12 & 5.48 & 7.20 & 1.65 & 3.07 & 1.52 & 3.75 \\
\hline B-1-h & $32-3,80-90$ & 56.00 & 0.96 & 13.30 & 2.90 & 3.02 & 0.12 & 2.72 & 5.82 & 0.12 & 0.20 & 0.10 & 4.26 & 9.54 & 5.22 & 2.45 & 1.70 & 11.87 \\
\hline B-2 & $3-2,74-87$ & 53.64 . & 0.95 & 13.19 & 2.30 & 3.40 & 0.11 & 3.14 & 6.75 & 0.12 & 1.52 & 0.07 & 5.23 & 10.15 & 4.86 & 2.33 & 1.84 & 11.05 \\
\hline B-2 & $9-2,128-142$ & 50.27 & 1.12 & 16.89 & 2.30 & 4.48 & 0.09 & 3.16 & 5.27 & 0.13 & 0.06 & 0.03 & 4.94 & 10.15 & 6.15 & 3.39 & 1.78 & 13.99 \\
\hline B-3 & $15-3,26-36$ & 49.35 & 1.01 & 16.18 & 3.39 & 2.44 & 0.07 & 2.15 & 7.64 & 0.09 & 0.50 & 0.008 & 4.36 & 11.70 & 7.30 & 2.65 & 1.90 & 16.60 \\
\hline B-3 & $30-1,108-120$ & 52.45 & 1.02 & 17.95 & 2.78 & 4.49 & 0.09 & 2.51 & 1.43 & 0.09 & 2.30 & 0.11 & 5.76 & 9.30 & 3.45 & 2.92 & 2.14 & 7.84 \\
\hline B-3 & $34-5,112-123$ & 51.75 & 0.96 & 17.85 & 3.02 & 3.13 & 0.12 & 2.43 & 3.62 & 0.07 & 0.58 & 0.14 & 6.76 & 11.80 & 4.68 & 2.42 & 1.94 & 10.64 \\
\hline B-4-a & $37-3,0-12$ & 55.65 & 0.97 & 20.15 & 2.90 & 3.49 & 0.12 & 1.72 & 0.99 & 0.09 & 0.16 & 0.01 & 6.88 & 8.55 & 1.64 & 2.59 & 1.94 & 3.73 \\
\hline \multicolumn{19}{|c|}{ Hole $380 \mathrm{~A}$} \\
\hline B-4-a & $40-5,1-17$ & 56.94 & 0.94 & 19.22 & 1.93 & 4.53 & 0.08 & 1.74 & 0.84 & 0.17 & 0.22 & 0.02 & 6.34 & 7.46 & 1.40 & 2.52 & 2.41 & 3.18 \\
\hline$B-4-b$ & $50-2,130-140$ & 18.82 & 0.50 & 5.65 & 1.21 & 1.52 & 0.11 & 2.52 & 33.45 & 0.13 & 0.75 & 0.06 & 0.78 & 31.85 & 31.00 & 1.12 & 2.14 & 70.50 \\
\hline$B-4-e$ & $62-3,0-15$ & 54.06 & 1.02 & 16.68 & 2.21 & 3.08 & 0.05 & 2.21 & 3.63 & 0.12 & 1.22 & 0.06 & 5.64 & 9.85 & 4.16 & 3.10 & 2.59 & 9.46 \\
\hline B-5 & $71-3,57-68$ & 37.44 & 0.79 & 14.57 & 2.42 & 2.50 & 0.11 & 5.02 & 11.24 & 0.16 & 1.51 & 0.06 & 5.72 & 19.50 & 13.74 & 1.94 & 2.33 & 31.25 \\
\hline \multicolumn{19}{|c|}{ Site 381} \\
\hline 3 & $3-1,120-145$ & 51.47 & 0.98 & 16.08 & 1.09 & 5.22 & 0.12 & 3.02 & 5.93 & 0.18 & 0.99 & 0.04 & 4.73 & 10.40 & 5.50 & 2.84 & 1.71 & 12.73 \\
\hline 3 & $10-1,125-150$ & 48.77 & 1.05 & 19.21 & 1.89 & 5.27 & 0.08 & 2.22 & 3.94 & 0.30 & 0.07 & 0.04 & 8.20 & 11.20 & 2.96 & 3.01 & 2.35 & 6.73 \\
\hline 4 & $18-2,0-15$ & 64.11 & 0.44 & 6.45 & 1.33 & 2.18 & 0.06 & 0.82 & 9.75 & 0.10 & 0.90 & 0.04 & 2.02 & 10.81 & 8.72 & 1.62 & 0.97 & 19.83 \\
\hline 5 & $19-2,92-102$ & 52.28 & 1.02 & 20.72 & 2.48 & 5.27 & 0.09 & 2.46 & 0.42 & 0.10 & 0.15 & 0.01 & 6.78 & 9.45 & 2.64 & 2.14 & 2.65 & 6.00 \\
\hline 5 & $24-1,48-63$ & 58.94 & 0.94 & 16.24 & 1.45 & 4.34 & 0.09 & 2.46 & 0.42 & 0.09 & 0.30 & 0.11 & 6.78 & 9.50 & 2.56 & 2.38 & 2.90 & 5.82 \\
\hline 6 & $34, \mathrm{CC}$ & 39.29 & 0.61 & 10.15 & 1.63 & 7.12 & 0.28 & 2.61 & 14.07 & 0.08 & 4.78 & 0.13 & 4.88 & 11.05 & 10.02 & 1.45 & 2.68 & 22.79 \\
\hline 8 & $42-1,90-100$ & 57.37 & 0.27 & 6.28 & 0.60 & 0.78 & 0.04 & 3.25 & 13.85 & 0.08 & 0.33 & tr & 0.64 & 13.81 & 13.12 & 1.60 & 1.69 & 29.84 \\
\hline 9 & $49-2,70-83$ & 53.13 & 1.02 & 17.86 & 2.66 & 4.59 & 0.09 & 3.36 & 1.69 & 0.11 & 1.70 & 0.05 & 5.80 & 8.99 & 3.12 & 2.73 & 1.96 & 7.10 \\
\hline 10 & $51-5,0-12$ & 53.42 & 0.98 & 18.47 & 3.02 & 2.53 & 0.09 & 3.84 & 4.95 & 0.12 & 0.06 & tr & 4.62 & 6.70 & 2.08 & 3.00 & 2.62 & 4.73 \\
\hline 11 & $53-2,19-29$ & 57.82 & 0.95 & 17.94 & 3.87 & 1.59 & 0.09 & 2.74 & 1.55 & 0.17 & 0.06 & 0.06 & 5.38 & 9.84 & 4.40 & 1.70 & 1.80 & 10.01 \\
\hline
\end{tabular}

379 and Hole 380 . The carbonates from Hole 380A are characterized by alternations of high-calcareous and low to non-carbonaceous contents (with almost zero $\mathrm{CaCO}_{3}$ content, Table 13).

Early Pleistocene sediments are characterized by the presence of numerous beds high in carbonate. These beds were seldom found in Unit B-2, whereas in Unit B3 , they are common (Table 13). Calcite is generally dominant, where dolomite and siderite are subordinate and found only sporadically. Different associations of carbonate minerals occur: calcite-dolomite-siderite, calcite-dolomite, calcite-siderite, and (rarely) dolomitesiderite. Beds with high $\mathrm{CaCO}_{3}$ contents consist almost exclusively of calcite and contain a small admixture of siderite (up to $3.30 \%$ ). The presence or absence of dolomite and siderite was found to have no connection with quantitative fluctuations, either of $\mathrm{CaCO}_{3}$ or of $\mathrm{C}_{\text {org }}$.

In the Glacial series of the early Pleistocene sediments (Unit B-3), carbonates are mainly represented by calcite; siderite is found sporadically. It is of subordinate importance, though its content here is sometimes higher than it is in Unit B-2. Dolomite was observed in a few interbeds. Calcite content in a number of interbeds is high $(51-83 \%$ of the crystalline phase), and those beds with high carbonate content consist almost exclusively of calcite.
In the Pliocene sediments, a frequent alternation of layers of high and low (almost zero) carbonate content was observed. Carbonates are represented by calcite and siderite in Unit B-3. In the high-carbonate layers, calcite is dominant where siderite is present only as an admixture. The low-carbonate muds enriched in organic matter were chemically analyzed and found to contain almost zero $\mathrm{CaCO}_{3}$ content; only siderite was observed, its content reaching $10 \%$. Interbeds of lithified siderite were also found $(40-2,148-149 \mathrm{~cm}$; 41$6,108-122 \mathrm{~cm}$, etc.). One of these siderite interbeds (40$2,148-149 \mathrm{~cm}$ ) was studied in thin section. A relict structure is suggestive of siderite having replaced a diatomaceous mud.

A sample (from Unit 4d) of calcareous rock with a relict organogenic structure composed of breccia and other clastic lithified sediments, was analyzed by X-ray and in thin section. It mainly consists of calcite and contains ankerite which probably replaced calcite. Recrystallized shells of organisms were seen in thin section.

Unit 4-e is characterized by the presence of lowsiliceous diatomaceous carbonate muds. The carbonate mineral composition is sharply changed. In addition to calcite, dolomite occurs and is locally dominant, reaching almost $40 \%$; siderite is absent. Large amounts of aragonite (23.1-48.4\%) were sporadically observed. 
TABLE 13

Chemical Composition of the Bottom Sediments, Hole 380A

Content $\left(10^{-4} \%\right)$

\begin{tabular}{|c|c|c|c|c|c|c|c|c|c|c|c|c|c|c|c|c|c|c|c|c|c|c|c|}
\hline \multirow{2}{*}{$\begin{array}{l}\text { Depth } \\
(\mathrm{m})\end{array}$} & \multirow[b]{2}{*}{$\begin{array}{c}\text { Sample } \\
\text { (Interval in cm) }\end{array}$} & & & & & & & & & & \\
\hline & & $\mathrm{CaCO}_{3}$ & $\mathrm{C}_{\text {org }}$ & $\begin{array}{r}\mathrm{SiO}_{2} \\
\times \quad 1.6\end{array}$ & $\mathrm{Fe}$ & $\mathrm{Mn}$ & $\mathrm{Ti}$ & $\mathbf{P}$ & K & $\mathrm{Na}$ & $\mathrm{Cu}$ & $\mathrm{Zn}$ & $\mathrm{Ni}$ & Co & $\mathrm{Cr}_{\mathrm{r}}$ & $\mathrm{Cd}$ & $\mathrm{Li}$ & $\mathrm{Rb}$ & $\mathrm{Cs}$ & $\mathrm{Pb}$ & $\mathrm{Sn}$ & $\mathrm{F}$ & $\mathrm{B}_{\text {chem }}$ \\
\hline 335.95 & $1-3,45-60$ & 1.00 & - & - & $(4.6)^{\mathrm{a}}$ & - & - & - & - & - & - & - & - & $(4.0)$ & - & - & - & - & - & - & - & 860 & - \\
\hline 353.74 & $3-2,74-87$ & 9.22 & 1.32 & 2.93 & 4.93 & 0.09 & 0.43 & 0.05 & 2.12 & 1.54 & 62 & 95 & 74 & (14) & 113 & $<4$ & 25 & 60 & 5.0 & 25 & 2.6 & 820 & 129 \\
\hline 356.46 & $3-4,46-61$ & 6.50 & - & - & - & - & - & - & - & - & - & - & - & - & - & - & - & - & - & - & - & - & - \\
\hline 361.32 & $4-1,32-45$ & 10.41 & 0.31 & 1.38 & 4.34 & 0.06 & 0.51 & 0.06 & 2.69 & 1.45 & 37 & 95 & 68 & - & 117 & $<5$ & 25 & 55 & 4.5 & 22 & 2.2 & - & - \\
\hline 364.60 & $4-3,60-79$ & 12.00 & - & - & - & - & - & - & - & - & - & - & - & - & - & - & - & - & - & - & - & - & - \\
\hline 365.83 & $4-4,33-48$ & 6.51 & 0.27 & - & - & - & - & - & - & - & - & - & - & - & - & - & - & - & - & - & - & - & - \\
\hline 367.38 & $4-5,38-51$ & 11.02 & 0.34 & 1.03 & 4.84 & 0.08 & 0.46 & 0.06 & 2.56 & 1.22 & 43 & 84 & 61 & - & 125 & $<4$ & 30 & 70 & 6.0 & 20 & 2.0 & 760 & - \\
\hline 370.50 & $4, \mathrm{CC}$ & 7.00 & - & - & - & - & - & - & - & - & - & - & - & - & - & - & - & - & - & - & - & - & - \\
\hline 370.50 & $5-1,0-15$ & 7.00 & - & - & - & - & - & - & - & - & - & - & - & - & - & - & - & - & - & - & - & 860 & - \\
\hline 375.61 & $5-4,61-74$ & 11.50 & 0.34 & 0.96 & 4.97 & 0.09 & 0.47 & 0.06 & 2.49 & 1.18 & 42 & 94 & 57 & - & 125 & $<4$ & 35 & 60 & 5.0 & 15 & 1.6 & - & - \\
\hline 380.00 & $5, \mathrm{CC}$ & 13.00 & - & - & - & - & - & - & - & - & - & - & - & - & - & - & - & - & - & - & - & - & - \\
\hline 384.06 & $6-3,106-119$ & 24.45 & 0.76 & 1.04 & 4.48 & 0.21 & 0.38 & 0.08 & 1.66 & 1.39 & 46 & 84 & 57 & - & 102 & $<4$ & 20 & 55 & 5.0 & 10 & 1.0 & - & - \\
\hline 384.50 & $6-4,0-18$ & 19.00 & 0.60 & 1.11 & 4.66 & 0.14 & 0.43 & 0.07 & 2.16 & 1.54 & 50 & 94 & 88 & - & 117 & $<5$ & 18 & 50 & 5.0 & 12 & 1.0 & 540 & 112 \\
\hline 402.61 & $8-3,61-63$ & 9.26 & 0.54 & - & - & - & - & - & - & - & - & - & - & - & - & - & - & - & - & - & - & - & - \\
\hline 404.79 & $8-4,129-150$ & 6.41 & 0.69 & 1.46 & 5.29 & 0.04 & 0.55 & 0.07 & 2.57 & 2.07 & 34 & 86 & 57 & - & 122 & $<4$ & 18 & 60 & 4.6 & 15 & 2.2 & - & - \\
\hline 411.28 & $9-2,128-142$ & 9.40 & 0.41 & 1.50 & 5.56 & 0.07 & 0.51 & 0.07 & 2.91 & 1.66 & 37 & 94 & 76 & - & 118 & $<5$ & 22 & 70 & 5.0 & 15 & 1.4 & - & - \\
\hline 412.22 & $9-3,72-84$ & 15.65 & 0.60 & 1.31 & 5.11 & 0.09 & 0.51 & 0.07 & 2.32 & 0.94 & 33 & 81 & 55 & - & 114 & $<5$ & 22 & 90 & 6.0 & 18 & 2.0 & - & - \\
\hline 415.28 & $9-5,78-95$ & 3.89 & 0.27 & 1.37 & 4.39 & 0.06 & 0.50 & 0.08 & 2.52 & 1.72 & 50 & 94 & 64 & - & 116 & $<4$ & 50 & 70 & 6.0 & 20 & 2.0 & - & - \\
\hline 416.97 & $9-6,97-106$ & 55.34 & 0.50 & 0.64 & 4.81 & 0.23 & 0.20 & 0.14 & 2.65 & 0.99 & 33 & 40 & 46 & - & 38 & $<5$ & 14 & 30 & 3.0 & 4 & - & 770 & - \\
\hline 420.12 & $10-2,62-74$ & 13.83 & 0.29 & 0.96 & 5.15 & 0.16 & 0.47 & 0.07 & 1.93 & 0.95 & 37 & 94 & 63 & (14) & 120 & $<5$ & 60 & 90 & 7.0 & 18 & 2.2 & - & - \\
\hline 429.99 & $11-2,99-115$ & 16.50 & - & - & - & - & - & - & - & - & - & - & - & - & - & - & - & - & - & - & - & 720 & - \\
\hline 431.44 & $11-3,94-106$ & 14.00 & - & - & - & - & - & - & - & - & - & - & - & - & - & - & - & - & - & - & - & - & - \\
\hline 435.58 & $11-6,58-69$ & 3.45 & 0.36 & 1.60 & 5.29 & 0.07 & 0.50 & 0.04 & 2.57 & 2.17 & 48 & 120 & 55 & - & 124 & $<4$ & 50 & 80 & 5.0 & 25 & 2.4 & - & - \\
\hline 438.51 & $12-2,1-17$ & 7.50 & - & - & - & - & - & - & - & - & - & - & - & - & - & - & - & - & - & - & - & - & - \\
\hline 441.50 & $12-4,0-16$ & 36.86 & 0.52 & 1.29 & 3.14 & 0.09 & 0.32 & 0.05 & 1.16 & 1.19 & 45 & 70 & 51 & - & 76 & $<5$ & 25 & 50 & 5.0 & 10 & 1.0 & 370 & 78 \\
\hline 444.99 & $12-6,49-61$ & 11.72 & 0.26 & 1.33 & 4.25 & 0.06 & 0.44 & 0.04 & 2.22 & 1.91 & 51 & 97 & 55 & - & 111 & $<4$ & 35 & 55 & 4.5 & 18 & 1.3 & - & - \\
\hline Average & & & 0.49 & 1.33 & 4.74 & 0.10 & 0.44 & 0.07 & 2.30 & 1.46 & 43 & $8 r$ & 62 & - & 109 & $<4$ & 30 & 63 & 5.1 & 16 & 1.8 & 705 & 106 \\
\hline 447.50 & $13-1,110-111$ & 13.82 & 0.46 & 1.00 & 4.03 & 0 . & 0.28 & 0.05 & - & - & - & - & - & - & - & - & 45 & 60 & 5.0 & 16 & 1.8 & - & - \\
\hline 458.12 & $14-2,62$ & 13.61 & 0.29 & 1.19 & 4.03 & 0.06 & 0.48 & 0.05 & 1.88 & 1.15 & 52 & 96 & 71 & - & 120 & $<5$ & 40 & 60 & 4.5 & 17 & 1.3 & - & - \\
\hline 459.15 & $14-3,15-28$ & 41.92 & 0.56 & 0.90 & 3.23 & 0.11 & 0.31 & 0.05 & 2.31 & 1.48 & 39 & 69 & 40 & - & 67 & $<4$ & - & - & - & - & - & - & - \\
\hline 460.36 & $14-3,136-147$ & 61.00 & - & - & - & - & - & - & - & - & - & - & - & - & - & - & 25 & 40 & 3.0 & 10 & 1.0 & 490 & - \\
\hline 464.17 & $14-6,67-79$ & 26.88 & 0.61 & 1.15 & 3.85 & 0.16 & 0.45 & 0.05 & 1.89 & 0.89 & 38 & 97 & 73 & - & 108 & $<5$ & 50 & 60 & 5.0 & 15 & 1.4 & - & - \\
\hline 468.76 & $15-3,26-36$ & 14.28 & 0.46 & 1.35 & 4.06 & 0.07 & 0.46 & 0.05 & 2.08 & 1.52 & 59 & 104 & 57 & - & 94 & $<4$ & 50 & 60 & 5.0 & 18 & 2.0 & 550 & - \\
\hline 475.00 & $15, \mathrm{CC}$ & 2.3 & - & - & - & - & - & - & - & - & - & - & - & - & - & - & - & - & - & - & - & - & - \\
\hline 486.56 & $17-2,56-70$ & 54.70 & 0.48 & 1.09 & 2.60 & 0.08 & 0.25 & 0.01 & 1.54 & 1.19 & 28 & 55 & 66 & - & 38 & $<5$ & 18 & 50 & 4.5 & 6 & 1.0 & - & - \\
\hline 487.11 & $17-2,111-123$ & 1.75 & 0.50 & 4.68 & 4.56 & 0.04 & 0.46 & 0.03 & 2.33 & 1.75 & 60 & 120 & 52 & - & 103 & $<4$ & - & - & - & - & - & - & - \\
\hline 487.64 & $17-3,14-29$ & 5.00 & - & - & - & - & - & - & - & - & - & - & - & - & - & - & - & - & - & - & - & 730 & - \\
\hline 495.94 & $18-2,44-59$ & 10.50 & - & - & - & - & - & - & - & - & - & - & - & - & - & - & - & - & - & - & - & 500 & 115 \\
\hline 497.33 & $18-3,33-48$ & 42.05 & 0.39 & 0.90 & 3.67 & 0.20 & 0.28 & 0.05 & 1.39 & 1.14 & 35 & 67 & 40 & - & 60 & $<4$ & 30 & 55 & 5.0 & 14 & 1.2 & - & - \\
\hline 505.00 & $19-2,0-16$ & 14.12 & 0.52 & 1.48 & 4.43 & 0.03 & 0.40 & 0.06 & 2.03 & 1.22 & 55 & 80 & 68 & - & 120 & $<5$ & 30 & 60 & 5.0 & 20 & 1.6 & - & - \\
\hline 508.00 & $19-4,0-16$ & 33.48 & 0.68 & 1.48 & 3.75 & 0.20 & 0.32 & 0.07 & - & - & - & - & - & - & - & - & 30 & 70 & 5.6 & 20 & 1.6 & - & - \\
\hline 514.89 & $20-2,39-51$ & 11.02 & 0.60 & 1.64 & 4.85 & 0.08 & 0.45 & 0.06 & 1.96 & 1.19 & 47 & 72 & 68 & - & 90 & $<5$ & 40 & 70 & 6.0 & 25 & 2.0 & - & - \\
\hline 517.50 & $20-4,0-13$ & 19.00 & - & - & - & - & - & - & - & - & - & - & - & - & - & - & - & - & - & - & - & 850 & - \\
\hline 532.00 & $21, \mathrm{CC}$ & 39.50 & - & - & - & - & - & - & - & - & - & - & - & - & - & - & - & - & - & - & - & - & - \\
\hline 535.01 & $22-3,1-12$ & 29.00 & - & - & - & - & - & - & - & - & - & - & - & - & - & - & - & - & - & - & - & 820 & - \\
\hline 535.84 & $22-3,84-96$ & 49.87 & 0.82 & 0.95 & 2.93 & 0.09 & 0.23 & 0.07 & 1.39 & 1.80 & 34 & 80 & 37 & (10) & 60 & $<4$ & 30 & 60 & 6.0 & 10 & 1.0 & - & - \\
\hline 542.20 & $23-1,70-85$ & 16.50 & - & - & - & - & - & - & - & - & - & - & - & - & - & - & - & - & - & - & - & 820 & - \\
\hline 544.71 & $23-3,21-33$ & 50.58 & 0.50 & 0.94 & 2.53 & 0.09 & 0.22 & 0.05 & 2.36 & 0.96 & 31 & 51 & 43 & - & 50 & $<5$ & 25 & 50 & 4.5 & 7 & 1.0 & - & - \\
\hline 548.27 & $23-5,77-87$ & 47.22 & 0.49 & 0.97 & 3.44 & 0.13 & 0.23 & 0.06 & 2.16 & 1.01 & 30 & 57 & 44 & - & 56 & $<5$ & 28 & 50 & 4.0 & 6 & 1.0 & - & - \\
\hline 551.00 & $23, \mathrm{CC}$ & 36.50 & - & - & - & - & - & - & - & - & - & - & - & - & - & - & - & - & - & - & - & - & - \\
\hline 562.45 & $25-2,45-61$ & 39.50 & 0.77 & 0.81 & 4.61 & 0.16 & 0.29 & 0.07 & 1.86 & 0.89 & 37 & 69 & 62 & (12) & 76 & $<5$ & 35 & 60 & 5.2 & 12 & 1.2 & - & - \\
\hline 563.26 & $25-2,126-136$ & 6.95 & 0.58 & 1.34 & 5.32 & 0.10 & 0.45 & 0.04 & 2.02 & 1.13 & 51 & 92 & 81 & - & 126 & $<5$ & 40 & 60 & 4.6 & 25 & 2.7 & - & - \\
\hline 563.74 & $25-3,24-40$ & 0.00 & 0.54 & - & - & - & - & - & - & - & - & - & - & - & - & - & - & - & - & - & - & - & - \\
\hline
\end{tabular}




\begin{tabular}{|c|c|c|c|c|c|c|c|c|c|c|c|c|c|c|c|c|c|c|c|c|c|c|c|}
\hline 572.21 & $26-2,71-87$ & 3.79 & 0.60 & 1.47 & 3.05 & 0.09 & 0.34 & 0.04 & 1.64 & 1.32 & 39 & 77 & 44 & - & 82 & $<4$ & 30 & 60 & 5.2 & 15 & 1.2 & 310 & 112 \\
\hline 585.50 & $\begin{array}{l}27-5,0-8 \\
27, \mathrm{CC}\end{array}$ & $\begin{array}{l}12.00 \\
24.50\end{array}$ & - & - & - & - & - & - & - & - & - & - & - & - & - & - & - & - & - & - & - & - & $\overline{-}$ \\
\hline 589.00 & $\begin{array}{l}27, \mathrm{CC} \\
29-6,0-25\end{array}$ & $\begin{array}{l}24.50 \\
48.90\end{array}$ & & - & - & - & - & - & - & - & - & - & - & - & - & - & - & - & - & - & - & - & $\overline{73}$ \\
\hline 597.00 & $\begin{array}{l}29-6,0-25 \\
30-1,108-120\end{array}$ & $\begin{array}{r}48.90 \\
2.28\end{array}$ & $\begin{array}{l}0.60 \\
1.22\end{array}$ & $\overline{182}$ & - & - & - & - & - & - & - & - & - & - & - & - & - & - & - & - & - & 650 & 73 \\
\hline 599.58 & $30-1,108-120$ & 2.28 & 1.22 & 1.82 & 4.97 & 0.11 & 0.49 & 0.04 & 2.38 & 2.00 & 71 & 111 & 64 & - & 100 & $<4$ & 50 & 80 & 6.0 & 28 & 2.8 & - & - \\
\hline 600.38 & $30-2,38-50$ & 53.12 & 0.82 & 1.32 & 2.50 & 0.13 & 0.21 & 0.07 & 2.24 & 1.10 & 34 & 54 & 38 & - & 52 & $<5$ & 25 & 50 & 4.0 & 5 & 1.0 & - & - \\
\hline 608.00 & $30, \mathrm{CC}$ & 61.00 & - & - & - & - & - & - & - & - & - & $=$ & - & - & - & - & - & - & - & - & - & 720 & - \\
\hline 608.40 & $31-1,40-55$ & 1.46 & 0.57 & 1.40 & 5.90 & 0.10 & 0.49 & 0.04 & 3.04 & 2.37 & 66 & 116 & 64 & - & 100 & $<4$ & 50 & 70 & 5.0 & 28 & 2.5 & - & - \\
\hline 617.50 & $31, \mathrm{CC}$ & 30.50 & - & - & - & - & - & - & - & - & - & - & - & - & - & - & - & - & - & - & - & - & - \\
\hline 625.34 & $32-6,34-47$ & 54.84 & 0.60 & 1.12 & 4.15 & 0.16 & 0.21 & 0.06 & 1.08 & 0.99 & 30 & 52 & 48 & - & 42 & $<4$ & 20 & 45 & 4.0 & 5 & 1.0 & 670 & \\
\hline 627.00 & $32, \mathrm{CC}$ & 37.50 & - & - & - & - & - & - & - & - & - & - & - & - & - & - & - & - & - & - & - & - & - \\
\hline 639.77 & $34-3,27-44$ & 37.18 & 1.02 & 1.65 & 3.09 & 0.10 & 0.27 & 0.05 & 1.42 & 1.41 & 35 & 71 & 55 & (12) & 80 & $<4$ & 25 & 70 & 6.0 & 10 & 1.2 & 800 & - \\
\hline 646.00 & $34, \mathrm{C}$ & 63.50 & - & - & - & - & - & - & - & - & - & - & - & - & - & - & - & - & - & - & - & 770 & - \\
\hline Average & & & 0.63 & 1.44 & 3.92 & 0.11 & 0.35 & 0.05 & 1.96 & 1.37 & 44 & 80 & 56 & - & 112 & $<5$ & 34 & 61 & 5.0 & 15 & 1.5 & 668 & 100 \\
\hline 647.25 & $35-1,125-140$ & 0.00 & 0.90 & - & - & - & - & - & - & - & - & - & - & - & - & - & - & - & - & - & - & - & - \\
\hline 649.58 & $35-3,58-74$ & 2.94 & 1.52 & 2.12 & 5.28 & 0.03 & 0.45 & 0.04 & - & - & - & - & - & - & - & - & - & - & - & - & - & 850 & 82 \\
\hline 651.52 & $35-4,102-112$ & 1.00 & - & - & - & - & - & - & - & - & - & - & - & - & - & - & - & - & - & - & - & - & - \\
\hline 657.30 & $36-2,30-42$ & 1.00 & - & - & (3.9) & - & - & - & - & - & - & - & - & (12) & - & - & - & - & - & - & - & - & - \\
\hline 668.00 & $37-3,0-12$ & 1.00 & 0.67 & 2.14 & 4.49 & 0.04 & 0.46 & 0.04 & 2.29 & 1.91 & 68 & 124 & 72 & - & 104 & $<4$ & 50 & 120 & 7.0 & 21 & 2.4 & 770 & - \\
\hline 671.02 & $37-5,2-14$ & 1.00 & - & - & - & - & - & - & - & - & - & - & - & - & - & - & - & - & - & - & - & - & - \\
\hline 677.50 & $38-3,0-16$ & 0.43 & 1.34 & 2.91 & 4.70 & 0.11 & 0.47 & 0.03 & - & - & - & - & - & - & - & - & - & - & - & - & - & - & - \\
\hline 681.12 & $38-5,62-75$ & 0.46 & 1.10 & 2.95 & 4.79 & 0.05 & 0.46 & 0.03 & 2.11 & 1.55 & 68 & 113 & 78 & - & 115 & 4 & 40 & 90 & 6.0 & 22 & 2.4 & - & - \\
\hline 698.06 & $40-4,6-20$ & 1.00 & - & - & - & - & - & - & - & - & - & - & - & - & - & - & - & - & - & - & - & 820 & - \\
\hline 699.51 & $40-5,1-17$ & 0.36 & 0.67 & 3.97 & 4.65 & 0.04 & 0.45 & 0.04 & - & - & - & - & - & - & - & - & - & - & - & - & - & - & - \\
\hline 700.15 & $40-5,65-67$ & 0.00 & 0.99 & - & - & - & - & - & - & - & - & - & - & (7) & - & - & - & - & - & - & - & - & - \\
\hline 703.17 & $41-1,17-23$ & 1.00 & - & - & - & - & - & - & - & - & - & - & - & - & - & - & - & - & - & - & - & 440 & 191 \\
\hline 703.17 & $41-1,53-55$ & - & - & - & 33.10 & 3.36 & 0.23 & - & 0.34 & 0.30 & 22 & 32 & 55 & 6 & 38 & 10 & - & - & - & - & - & - & - \\
\hline 707.60 & $41-4,10-20$ & 0.40 & 1.72 & 11.87 & 3.04 & 0.02 & 0.33 & 0.02 & 1.52 & 2.12 & 54 & 64 & 72 & - & 88 & $<4$ & 22 & 40 & 3.0 & 14 & 1.5 & - & - \\
\hline 711.58 & $41-6,108-122$ & 0.0 & 1.85 & 9.76 & 3.20 & 0.03 & 0.36 & 0.03 & - & - & - & - & - & - & - & - & 25 & 60 & 4.0 & 20 & 2.0 & - & - \\
\hline 712.50 & $41, \mathrm{CC}$ & 1.00 & - & - & - & - & - & - & - & - & - & - & - & - & - & - & - & - & - & - & - & - & - \\
\hline 714.04 & $42-2,4-14$ & 1.00 & - & - & - & - & - & - & - & - & - & - & - & - & - & - & - & - & - & - & - & - & - \\
\hline 717.33 & $42-4,33-35$ & 48.04 & 0.69 & - & (16.3) & 1.02 & 0.23 & - & 0.71 & 1.09 & 19 & 40 & 43 & (10) & 58 & $<4$ & - & - & - & - & - & - & - \\
\hline Average & & & 1.19 & 5.09 & 8.21 & 0.50 & 0.39 & 0.03 & - & - & - & - & - & - & - & - & 35 & 76 & 5.0 & 19 & 2.1 & 720 & 136 \\
\hline 722.00 & $42, \mathrm{CC}$ & 5.00 & - & - & - & - & - & - & - & - & - & - & - & - & - & - & - & - & - & - & - & 460 & - \\
\hline 722.00 & $43-1,0-13$ & 0.00 & 3.18 & 9.25 & 4.41 & 0.06 & 0.34 & 0.05 & - & - & - & - & - & - & - & - & 40 & 120 & 4.5 & 18 & 2.0 & - & - \\
\hline 725.73 & $43-3,73-75$ & 2.00 & - & - & - & - & - & - & - & - & - & - & - & - & - & - & - & - & - & - & - & - & - \\
\hline 726.12 & $43-3,112-126$ & 9.45 & 2.37 & 8.54 & 4.18 & 0.06 & 0.34 & 0.04 & 1.60 & 1.70 & 55 & 55 & 62 & - & 95 & $<4$ & 28 & 70 & 5 & 15 & 1.8 & - & - \\
\hline 733.63 & $44-2,63$ & 14.02 & 1.74 & & $\overline{3} 67$ & 0,05 & & $0 \overline{03}$ & & & & 7 & $\overline{82}$ & - & & - & & & & & $=$ & - & I \\
\hline 738.80 & $44-5,130-140$ & 0.00 & 1.22 & 8.07 & $\begin{array}{l}3.67 \\
3.50\end{array}$ & $\begin{array}{l}0.05 \\
0.06\end{array}$ & $\begin{array}{l}0.39 \\
0.35\end{array}$ & $\begin{array}{l}0.03 \\
0.03\end{array}$ & 2.03 & $\begin{array}{l}1.83 \\
-\end{array}$ & 67 & 75 & 82 & - & 94 & $<4$ & 22 & 55 & 4.0 & 25 & 2.5 & - & - \\
\hline 745.12 & $45-3,112-125$ & 5.09 & 1.40 & 7.83 & 3.50 & $\begin{array}{c}0.06 \\
-\end{array}$ & 0.35 & 0.03 & $\bar{z}$ & - & - & - & - & - & - & - & 30 & 60 & 4.5 & 30 & 2.0 & 450 & - \\
\hline 752.55 & $46-2,55-57$ & 26.00 & - & - & $(3.4)$ & $\overline{0.10}$ & $0 . \overline{27}$ & 0.04 & $\overline{1.34}$ & $\overline{42}$ & $\overline{20}$ & - & - & (9) & - & - & - & - & - & - & - & - & $\overline{-}$ \\
\hline 753.51 & $46-3,1-10$ & 30.80 & 1.28 & 4.38 & 3.94 & $\begin{array}{l}0.10 \\
0.10\end{array}$ & 0.34 & 0.08 & $\begin{array}{l}1.34 \\
1.76\end{array}$ & 1.42 & 29 & 70 & 81 & - & 69 & $<4$ & 20 & 50 & 4.5 & 20 & 1.2 & - & $\overline{185}$ \\
\hline 760.50 & $47-1,50-60$ & 10.40 & 1.95 & 5.54 & 4.12 & 0.10 & -0.84 & 0.00 & 1.76 & 1.30 & 58 & 74 & 76 & - & 98 & $<4$ & 22 & 45 & 4.0 & 20 & 2.3 & 340 & $\begin{array}{l}185 \\
-\end{array}$ \\
\hline 769.50 & $47, \mathrm{CC}$ & 9.01 & 0.99 & - & - & $\overline{0 .} 1$ & 0.53 & 0.06 & $\overline{-}$ & - & - & - & - & - & - & - & $\overline{-}$ & - & - & - & - & - & - \\
\hline $\begin{array}{l}784.50 \\
788.00\end{array}$ & $\begin{array}{l}49-5,0-15 \\
49, \mathrm{CC}\end{array}$ & $\begin{array}{l}50.56 \\
35.50\end{array}$ & $\stackrel{0.86}{-}$ & $\begin{array}{l}2.86 \\
-\end{array}$ & 2.20 & - & - & - & - & - & - & - & - & - & - & - & - & - & - & - & - & - & - \\
\hline 789.38 & $50-1,138-150$ & - & - & - & - & - & - & - & - & - & - & - & - & - & - & - & - & - & - & - & - & 180 & 66 \\
\hline 790.80 & $50-2,130-140$ & 73.27 & 0.53 & 1.45 & 1.52 & 0.09 & 0.12 & 0.04 & 0.71 & 0.97 & 35 & 35 & 32 & - & 34 & $<4$ & 10 & 30 & 2.5 & 3 & - & - & - \\
\hline 800.96 & $51-3,46-60$ & 42.00 & - & - & - & - & - & - & - & - & - & - & - & - & - & - & - & - & - & - & - & - & 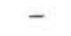 \\
\hline 802.34 & $51-4,34-50$ & 50.11 & 0.38 & 2.33 & 2.08 & 0.06 & 0.22 & 0.08 & - & - & - & - & - & - & - & - & 16 & 40 & 4.0 & 5 & 1.0 & - & - \\
\hline 805.01 & $51-6,1-12$ & 41.00 & - & - & - & - & - & - & - & - & - & - & - & - & - & - & - & - & - & - & - & - & - \\
\hline 809.00 & $52-2,0-12$ & 48.39 & 0.32 & 2.17 & 2.68 & 0.09 & 0.24 & 0.05 & 1.86 & 1.16 & 24 & 37 & 30 & $\overline{-}$ & 48 & $<4$ & 25 & 80 & 6.0 & 5 & 1.0 & - & - \\
\hline 816.21 & $52-6,121-123$ & 39.53 & 1.23 & - & (2.6) & - & - & - & - & - & - & - & - & (9) & -. & - & - & - & - & - & - & - & - \\
\hline
\end{tabular}


TABLE 13 - Continued

\begin{tabular}{|c|c|c|c|c|c|c|c|c|c|c|c|c|c|c|c|c|c|c|c|c|c|c|c|}
\hline \multirow[b]{2}{*}{$\begin{array}{l}\text { Depth } \\
(\mathrm{m})\end{array}$} & \multirow[b]{2}{*}{$\begin{array}{c}\text { Sample } \\
\text { (Interval in } \mathrm{cm} \text { ) }\end{array}$} & \multicolumn{9}{|c|}{ Content $(\%)$} & \multicolumn{13}{|c|}{ Content $\left(10^{-4} \%\right)$} \\
\hline & & $\mathrm{CaCO}_{3}$ & $\mathrm{C}_{\text {org }}$ & $\begin{array}{r}\mathrm{SiO}_{2} \\
\times 1.6\end{array}$ & $\mathrm{Fe}$ & $\mathrm{Mn}$ & $\mathrm{Ti}$ & $\mathbf{P}$ & K & $\mathrm{Na}$ & $\mathrm{Cu}$ & $\mathrm{Zn}$ & $\mathrm{Ni}$ & Co & $\mathrm{Cr}$ & $\mathrm{Cd}$ & $\mathrm{Li}$ & $\mathrm{Rb}$ & $\mathrm{Cs}$ & $\mathrm{Pb}$ & Sn & F & $\mathrm{B}_{\text {chem }}$ \\
\hline 818.51 & $53-2,1-15$ & 38.05 & 0.36 & 2.14 & 4.45 & 0.10 & 0.23 & 0.04 & 1.13 & 1.85 & 26 & 53 & 48 & - & 54 & $<4$ & 14 & 50 & 4.0 & 6 & 1.0 & - & - \\
\hline 820.48 & $53-3,48-64$ & 37.50 & - & - & - & - & - & - & - & - & - & - & - & - & - & - & - & - & - & - & - & 80 & 106 \\
\hline 827.16 & $54-1,66-78$ & 44.51 & 0.40 & 1.75 & 3.38 & 0.07 & 0.25 & 0.04 & - & - & - & - & - & - & - & - & 15 & 60 & 4.5 & 6 & 1.0 & - & - \\
\hline 839.55 & $55-3,55-57$ & 14.76 & 0.84 & - & - & - & - & - & - & - & - & - & - & - & - & - & - & - & - & - & - & - & - \\
\hline Average & & & 1.12 & 4.45 & 3.45 & 0.09 & 0.30 & 0.05 & 1.53 & 1.43 & 40 & 56 & 57 & - & 68 & $<4$ & 22 & 60 & 4.3 & 13 & 1.5 & 302 & 119 \\
\hline 840.71 & $55-4,21-32$ & 0.88 & 0.61 & 1.96 & 4.52 & 0.04 & 0.47 & 0.03 & - & - & - & - & - & (13) & - & - & - & - & - & - & - & - & - \\
\hline 845.50 & $55, \mathrm{CC}$ & 16.50 & - & - & - & - & - & - & - & - & - & - & - & - & - & - & - & - & - & - & - & - & - \\
\hline 846.45 & $56-1,95-105$ & 46.88 & 0.58 & 7.68 & 1.83 & 0.13 & 0.16 & 0.04 & - & - & - & - & - & - & - & - & 15 & 30 & 2.8 & 4 & - & 520 & 123 \\
\hline 849.05 & $56-3,58-60$ & 1.00 & - & - & (3.4) & - & - & - & - & - & - & - & - & (20) & - & - & - & - & - & - & - & - & - \\
\hline 855.00 & $57-1,0-15$ & 32.58 & 0.64 & 1.75 & 3.49 & 0.10 & 0.32 & 0.04 & - & - & - & - & - & - & - & - & - & - & - & - & - & - & - \\
\hline 857.03 & $57-2,53-62$ & 23.50 & - & - & - & - & - & - & - & - & - & - & - & - & - & - & - & - & - & - & - & 550 & - \\
\hline Average & & & 0.61 & 3.80 & 3.28 & 0.09 & 0.32 & 0.04 & - & - & - & - & - & - & - & - & 15 & 30 & 2.8 & 4 & - & 535 & 123 \\
\hline 864.86 & $58-1,36-39$ & - & - & - & - & - & - & - & - & - & - & - & - & - & - & - & - & - & - & - & - & - & - \\
\hline 875.17 & $59-1,117-118$ & - & - & - & 1.48 & 0.16 & 0.08 & - & - & - & - & - & - & - & - & - & - & - & - & - & - & - & - \\
\hline 883.50 & $59, \mathrm{CC}$ & 82.50 & - & - & - & - & - & - & - & - & - & - & - & - & - & - & - & - & - & - & - & - & - \\
\hline 883.63 & $60-1,13-19$ & 13.48 & 0.54 & 1.68 & 3.74 & 0.05 & 0.41 & 0.04 & 2.12 & 1.36 & 45 & 79 & 64 & - & 184 & $<4$ & 40 & 100 & 6.0 & 25 & 1.8 & - & - \\
\hline 886.51 & $60-3,11-12$ & 46.67 & 0.35 & 1.87 & 2.68 & 0.07 & 0.24 & 0.04 & - & - & - & - & - & - & - & - & 28 & 100 & 6.6 & 7 & 1.0 & - & - \\
\hline 888.65 & $60-4,65-80$ & 48.95 & 0.65 & 1.49 & 2.44 & 0.10 & 0.23 & 0.04 & - & - & - & - & - & - & - & - & 30 & 100 & 6.0 & 6 & 1.0 & - & - \\
\hline 893.00 & $60, \mathrm{CC}$ & 4.80 & - & - & - & - & - & - & - & - & - & - & - & - & - & - & - & - & - & - & - & - & - \\
\hline 904.00 & $62-2,0-15$ & 34.62 & 0.32 & 1.20 & 3.38 & 0.11 & 0.33 & 0.05 & - & - & - & - & - & - & - & - & 50 & 90 & 5.0 & 12 & 1.2 & 430 & 223 \\
\hline 905.50 & $62-3,0-15$ & 4.86 & 0.62 & 2.79 & 3.08 & 0.05 & 0.41 & 0.04 & - & - & - & - & - & - & - & - & 25 & 50 & 4.0 & 30 & 3.0 & - & - \\
\hline 913.50 & $63-2,0-15$ & 9.00 & - & - & - & - & - & - & - & - & - & - & - & - & - & - & - & - & - & - & - & - & - \\
\hline 916.50 & $63-4,0-15$ & 47.13 & 0.53 & 2.03 & 2.68 & 0.13 & 0.25 & - & - & - & - & - & - & - & - & - & 20 & 50 & 4.0 & 10 & 1.2 & - & - \\
\hline 926.69 & $64-4,20-22$ & - & - & - & 2.01 & 0.29 & 0.14 & - & - & - & - & - & - & - & - & - & - & - & - & - & - & - & - \\
\hline 926.69 & $64-4,69-80$ & 21.64 & 0.50 & 1.94 & 3.16 & 0.07 & 0.39 & - & - & - & - & - & - & - & - & - & 30 & 80 & 5.0 & 20 & 1.8 & - & - \\
\hline 931.00 & $64, \mathrm{CC}$ & 12.00 & - & - & - & - & - & - & - & - & - & - & - & - & - & - & - & - & - & - & - & - & - \\
\hline 933.02 & $65-2,52-56$ & - & - & - & 2.55 & 0.30 & 0.12 & - & - & - & - & - & - & - & - & - & - & - & - & - & - & - & - \\
\hline 934.00 & $65-3,0-15$ & 6.39 & 0.59 & 2.12 & 3.52 & 0.12 & 0.44 & - & - & - & - & - & - & - & - & - & 30 & 100 & 6.0 & 40 & 4.0 & - & - \\
\hline 950.11 & $67-1,11-13$ & 50.00 & - & - & - & - & - & - & - & - & - & - & - & - & - & - & - & - & - & - & - & - & - \\
\hline 972.72 & $69-3,72-74$ & - & - & - & 3.13 & 0.33 & 0.13 & - & - & - & - & - & - & - & - & - & - & - & - & - & - & - & - \\
\hline 972.86 & $69-3,86-100$ & 3.42 & 0.11 & 1.79 & 4.42 & 0.07 & 0.50 & - & - & - & - & - & - & - & - & - & 30 & 100 & 6.0 & 18 & 2.0 & 610 & - \\
\hline 974.03 & $69-4,53-58$ & - & - & - & 3.04 & 0.35 & 0.13 & - & 0.46 & 0.39 & 11 & 32 & $<10$ & - & 32 & $<4$ & - & - & - & - & - & - & - \\
\hline Average & & & 0.47 & 1.88 & 3.06 & 0.16 & 0.29 & 0.04 & 1.29 & 0.88 & 28 & 56 & 37 & - & 68 & $<4$ & 31 & 86 & 5.4 & 19 & 1.9 & 520 & 223 \\
\hline 982.54 & $70-3,104-107$ & - & - & - & 3.20 & 0.19 & 0.09 & - & - & - & - & - & - & - & - & - & - & - & - & - & - & - & - \\
\hline 985.71 & $70-5,121-122$ & - & - & - & 3.62 & 0.18 & 0.12 & - & - & - & - & - & - & - & - & - & - & - & - & - & - & - & - \\
\hline 989.63 & $71-2,13-21$ & 5.51 & 0.29 & 1.67 & 4.03 & 0.08 & 0.48 & - & - & - & - & - & - & (20) & - & - & 35 & 100 & 5.6 & 12 & 1.3 & 530 & 157 \\
\hline 991.57 & $71-3,57-68$ & 36.42 & 0.46 & 1.12 & 4.22 & 0.15 & 0.35 & - & - & - & - & - & - & - & - & - & 18 & 60 & 3.6 & 12 & 1.3 & - & - \\
\hline 1007.58 & $73-1,58-60$ & - & - & - & 3.76 & 0.15 & 0.07 & - & - & - & - & - & - & - & - & - & - & - & - & - & - & - & - \\
\hline 1008.55 & $73-2,5-18$ & 6.62 & 0.30 & 1.58 & 4.25 & 0.07 & 0.50 & - & - & - & - & - & - & - & - & - & 20 & 80 & 5.0 & 25 & 2.5 & - & - \\
\hline 1010.16 & $73-3,16-27$ & 4.69 & 0.16 & 1.09 & 4.64 & 0.08 & 0.49 & - & - & - & - & - & - & - & - & - & 22 & 80 & 5.0 & 25 & 2.5 & - & - \\
\hline 1018.70 & $74-2,70-83$ & 3.78 & 0.26 & 1.64 & 4.64 & 0.10 & 0.47 & - & - & - & - & - & - & - & - & - & 18 & 70 & 4.0 & 23 & 2.0 & - & - \\
\hline Average & & & 0.29 & 1.42 & 4.04 & 0.12 & 0.32 & - & - & - & - & - & - & - & - & - & 23 & 78 & 4.6 & 19 & 1.9 & 530 & 157 \\
\hline \multicolumn{3}{|c|}{$\begin{array}{l}\text { Average for hole (only sediments } \\
\text { with } \mathrm{CaCO}_{3} \text { contents }<30 \% \text { ) }\end{array}$} & 0.80 & 2.74 & 4.82 & 0.17 & 0.39 & 0.04 & 2.18 & 1.09 & 44 & 89 & 60 & - & 116 & $<5$ & 33 & 66 & 4.8 & 20 & 1.9 & 662 & 128 \\
\hline \multicolumn{3}{|c|}{$\begin{array}{l}\text { Average for hole (only sediments } \\
\text { with } \mathrm{CaCO}_{3} \text { contents }>30 \% \text { ) }\end{array}$} & 0.63 & 1.78 & 3.22 & 0.12 & 0.26 & 0.05 & 1.53 & 1.03 & 34 & 56 & 50 & - & 55 & $<5$ & 23 & 57 & 4.6 & 8 & 1.0 & 568 & 120 \\
\hline \multicolumn{3}{|c|}{ Average for hole } & 0.74 & 2.39 & 4.28 & 0.15 & 0.35 & 0.05 & 1.88 & 1.07 & 41 & 79 & 58 & 14 & 97 & $<5$ & 30 & 63 & 4.7 & 16 & 1.6 & 636 & 125 \\
\hline
\end{tabular}

${ }^{2}$ The data of INNA, not taken into account in the calculation of average contents. 
TABLE 14

Average Contents of Components and Elements in Different Units of Hole 380A

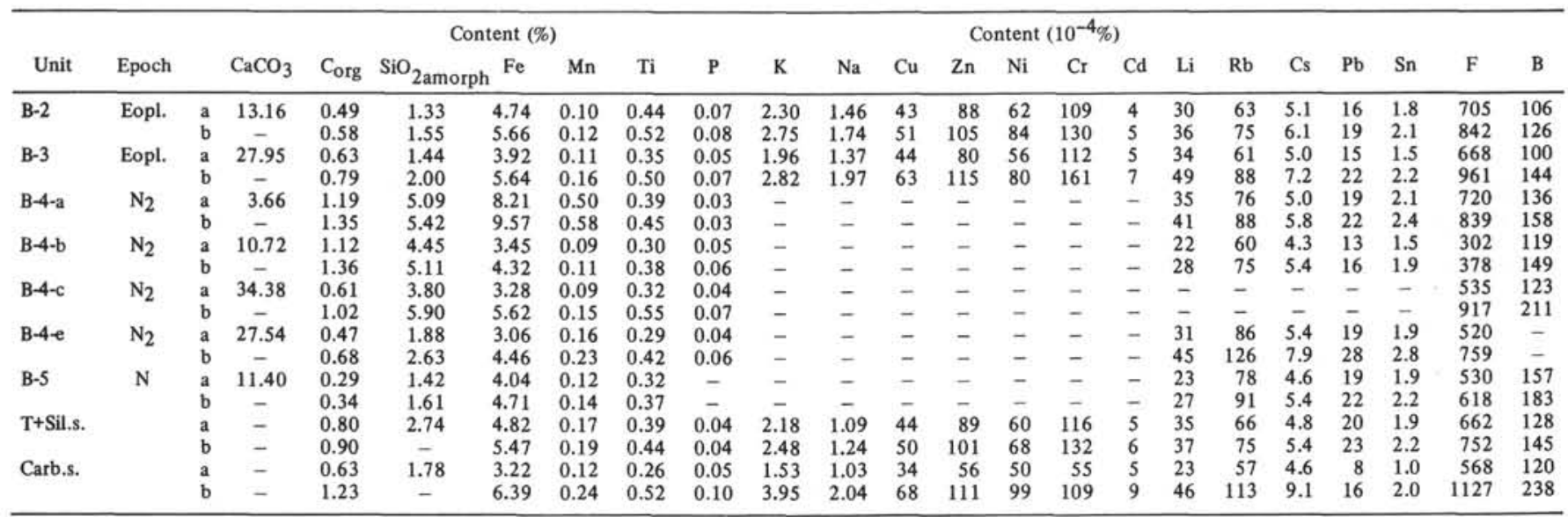

Note: $\mathrm{T}+\mathrm{Sil} . s .=$ terrigenous and siliceous biogenic sediments (i.e., sediments with $<30 \% \mathrm{CaCO}_{3}$, all hole); Carb.s. $=$ carbonate sediments (all sediments with $>30 \% \mathrm{CaCO} 3$ ); $\mathrm{a}=$ average in natural (dry) samples; $\mathrm{b}=$ average data according to the units on clastic matter basis (were calculated on average data only).

Thus in these low-siliceous diatomaceous-carbonate muds, a characteristic assemblage of carbonate minerals not found in other units at this site, was observed: calcite-dolomite-aragonite, with aragonite predominant. In other carbonate muds, dolomite is the dominant mineral. In oozes with $\mathrm{CaCO}_{3}$ contents $(<10 \%)$, carbonates are represented by calcite and, sometimes, dolomite.

The study of a number of lithified carbonate interbeds in thin section (Figure 15) showed that some of them are calcareous rocks with an aleuro-micrograined-pelitomorphous (inequigranular) structure, consisting of calcite-dolomite (64-4, 20-24 cm), and calcite with ankerite admixture $(65-2,52-56 \mathrm{~cm})$. Some of these interbeds contain clayey micrograined dolomite $(64-1,24-26 \mathrm{~cm} ; 69-3,72-74 \mathrm{~cm}$; 69-4, 53-58 $\mathrm{cm})$. This composition was confirmed by diffraction analysis. Clastic material (quartz, feldspars) are contained in the interbeds as a small admixture. Dolomite grains were observed to be rhombohedral, transparent, pure, and without any inclusions, which is taken as evidence of their authigenic nature. Sulfides and $\mathrm{Fe}$ hydroxide patches were ubiquitous.

The low-calcareous muds from Unit B-5 $\left(\mathrm{N}_{1}\right)$ contained more calcite. The lithified interbeds were observed to consist of clayey dolomite (70-5, 121-122 $\mathrm{cm}$; 73-1, 58-60 cm; 74-3, 107-109 cm). Thin section study (Figure 16) showed all of them to be characterized by microgranular (inequigranular) carbonate structures (grain sizes $<0.01 \mathrm{~mm}$ ). Some quartz and feldspar was present, corroded by carbonates. Finely dispersed sulfides were present. Pores were often filled with silica of opal-chalcedonic composition.

Site 381: The lower Pleistocene sediments, mainly of Glacial series, are characterized by sharp and frequent fluctuations of $\mathrm{CaCO}_{3}$ content. The upper limit of the values is not higher than $30 \%$, as a rule, excluding single interbeds. The lower limit approaches zero.

The carbonate mineral composition is similar to that of Hole 380A corresponding deposits, e.g., carbonates consist mainly of calcite, where dolomite and siderite are found sporadically.

In oozes with low $\mathrm{CaCO}_{3}$ contents $(<10$ and $<5 \%)$, only calcite was found. In these units, individual lithified carbonate interbeds were observed; only one was studied $(12-2,15-16 \mathrm{~cm})$. It is a finely laminated carbonate rock consisting of light and muddy brown bands, the brown ones reacting with $5 \% \mathrm{HCl}$. Thinsection observations showed the light bands to be a mixture of calcite and dolomite. In immersion slides, pure single dolomite crystals were seen, both rhombohedra and irregular crystals.

Thin sections showed the brown bands to comprise clayey microgranular carbonate (calcite, inequigranular) with larger siderite(?) (dolomite?) single crystals (regular and irregular rhombohedra) (Figure 17). Calcite (10\%) and siderite were identified by $X$-ray. Small amounts of quartz, feldspars, and micas occur.

In the Pliocene sediments, $\mathrm{CaCO}_{3}$ distribution is similar to that of Hole $380 \mathrm{~A}$, e.g., almost noncarbonate layers alternate with high-carbonate ones. The upper limit of values is $91.84 \%$.

In Unit $5\left(\mathrm{~N}_{2}\right)$, largely non-carbonate muds enriched in organic matter, dolomite almost disappears, whereas siderite is commonly found as discrete layers having a very high siderite content $(74.2-100 \%)(23-4,92-93 \mathrm{~cm}$; $27-3,77-79 \mathrm{~cm})$. In the thin section made from interbed $27-2,77-79 \mathrm{~cm}$, siderite (showing relict structure) formed by replacement of diatomaceous mud. Only occasional individual opaline diatom tests have remained (Figure 18).

In the low-carbonate and carbonate muds, calcite is the dominant carbonate mineral, with some admixture of siderite.

In Unit $6\left(\mathrm{~N}_{2}\right)$ carbonate and high-carbonate muds (30-50\% and $>50 \%$ of $\left.\mathrm{CaCO}_{3}\right)$ predominate, with total carbonate reaching $81.84 \%$. Beds with low to almost zero $\mathrm{CaCO}_{3}$ are also present. Carbonate and highcarbonate muds were found to be either enriched or impoverished in organic matter; some beds are also enriched in diatomaceous silica. Compared to Unit 5, 


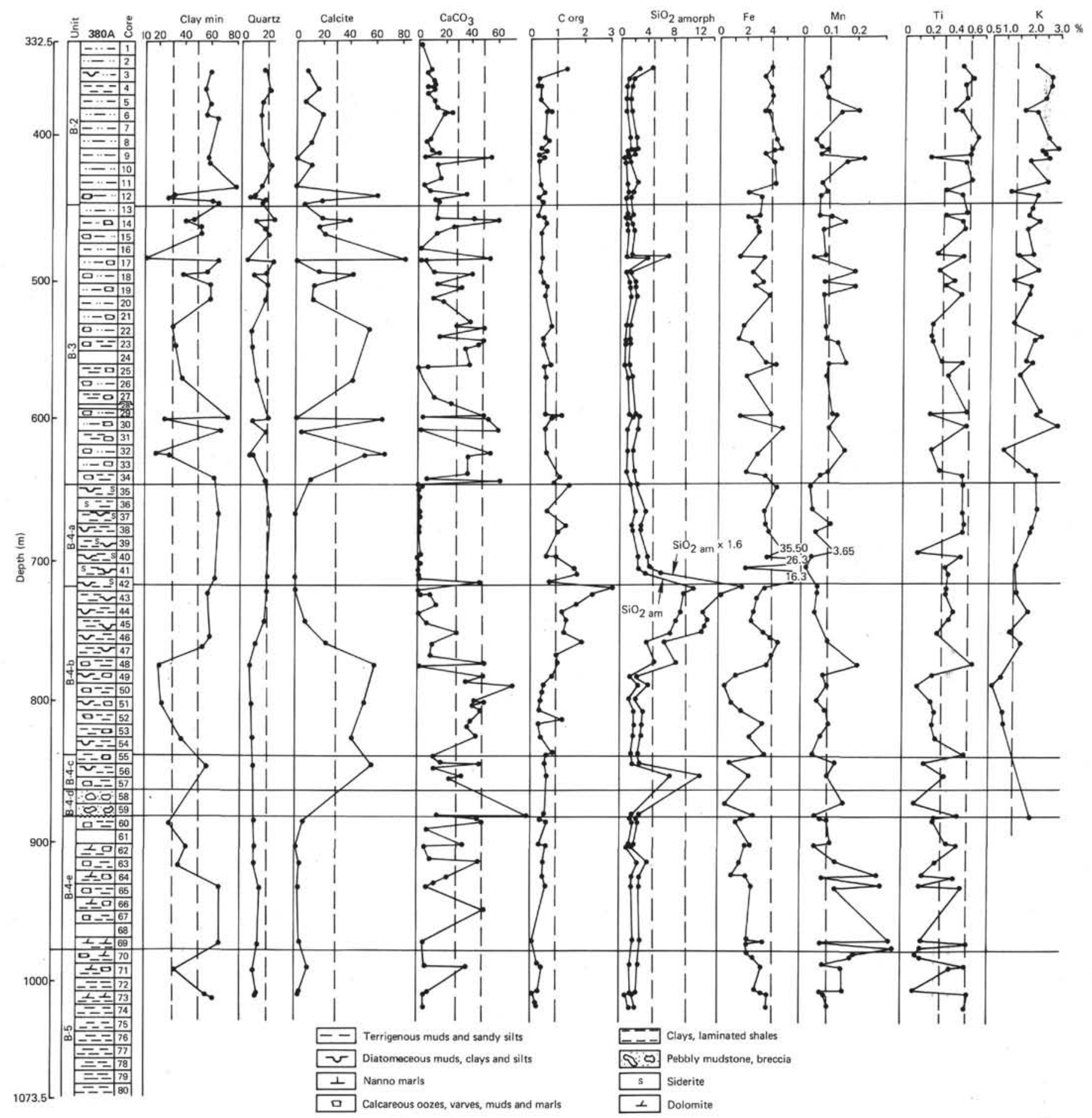

Figure 11. Lithologic composition of Hole $380 \mathrm{~A}$ sediments and distribution of main minerals and chemical macrocomponents.

calcite amounts here are sharply increased; layers enriched in aragonite were also found. Thus, in the beds of high-carbonate muds $(33-1,126-128 \mathrm{~cm} ; 33-4,120$ $135 \mathrm{~cm}$ ) enriched in organic matter, aragonite in addition to calcite is an important carbonate mineral. These same layers contain diatomaceous silica.

In the lower parts of Unit 6 and in Unit 7, the sediments contain abundant aragonite. This is true over a wide range of carbonate values $\left(9-81.8 \% \mathrm{CaCO}_{3}\right)$ and organic matter (0.58-6.56\% Corg); all aragonite-rich zones are, however, high in diatomaceous silica content. In addition to the usually dominant aragonite, calcite and, sporadically, dolomite occur. Maximum aragonite contents (70.7-77.8\%) occur in muds poor in organic matter, and highest in total $\mathrm{CaCO}_{3}$ (including aragonite) $(37-2,12-14 \mathrm{~cm} ; 37-5,56-58 \mathrm{~cm})$.

In the lower parts of Unit 7, the layers of lithified clay-carbonate muds consist mainly of dolomite (65.6\%) (30-1, 42-44 cm). Only a small admixture of calcite ( $2 \%)$ was found in this sample. Investigations of 


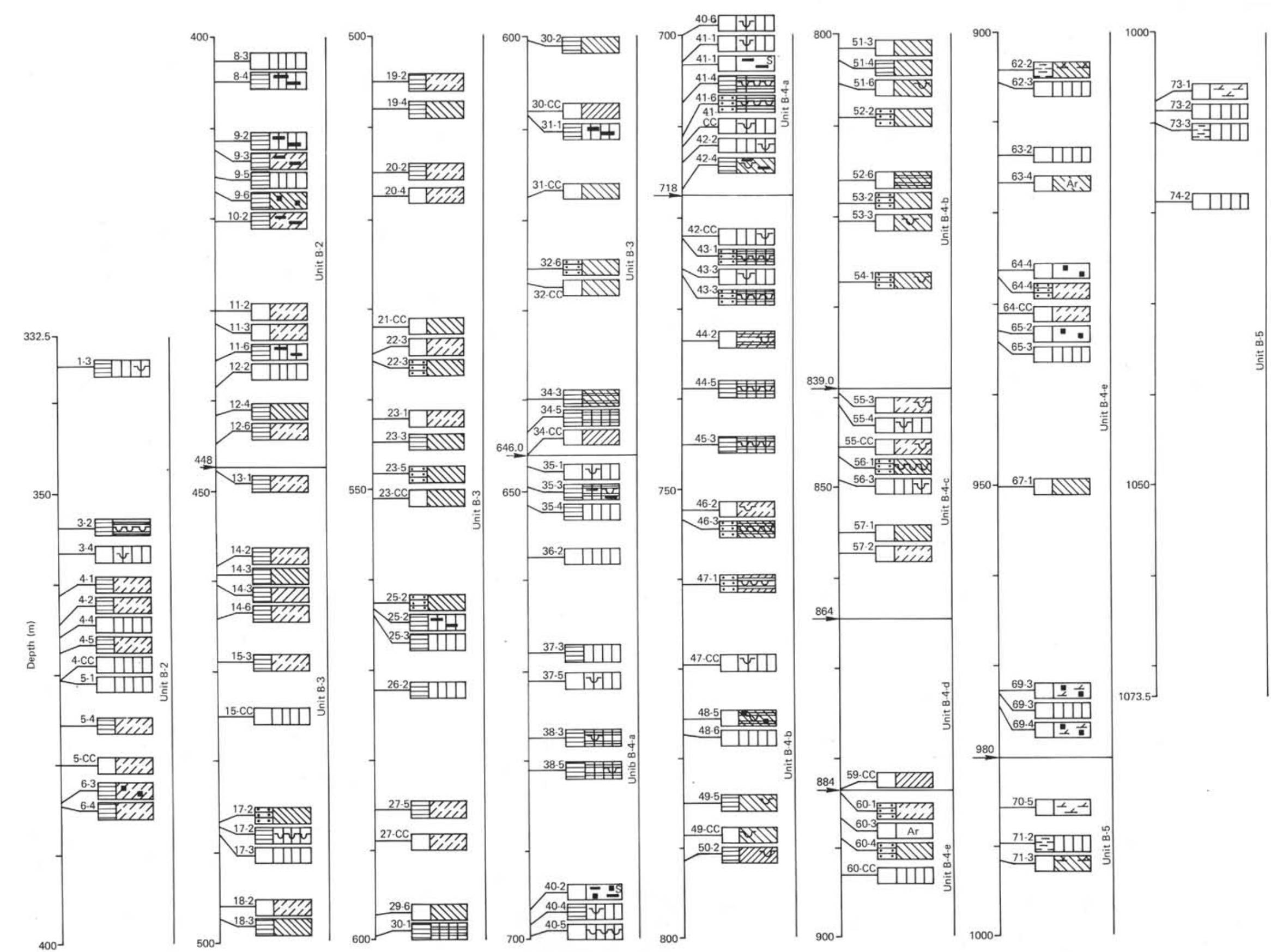

Un Figure 12. Composition of Hole 380A sediment samples. Designation as in Figure 4. 


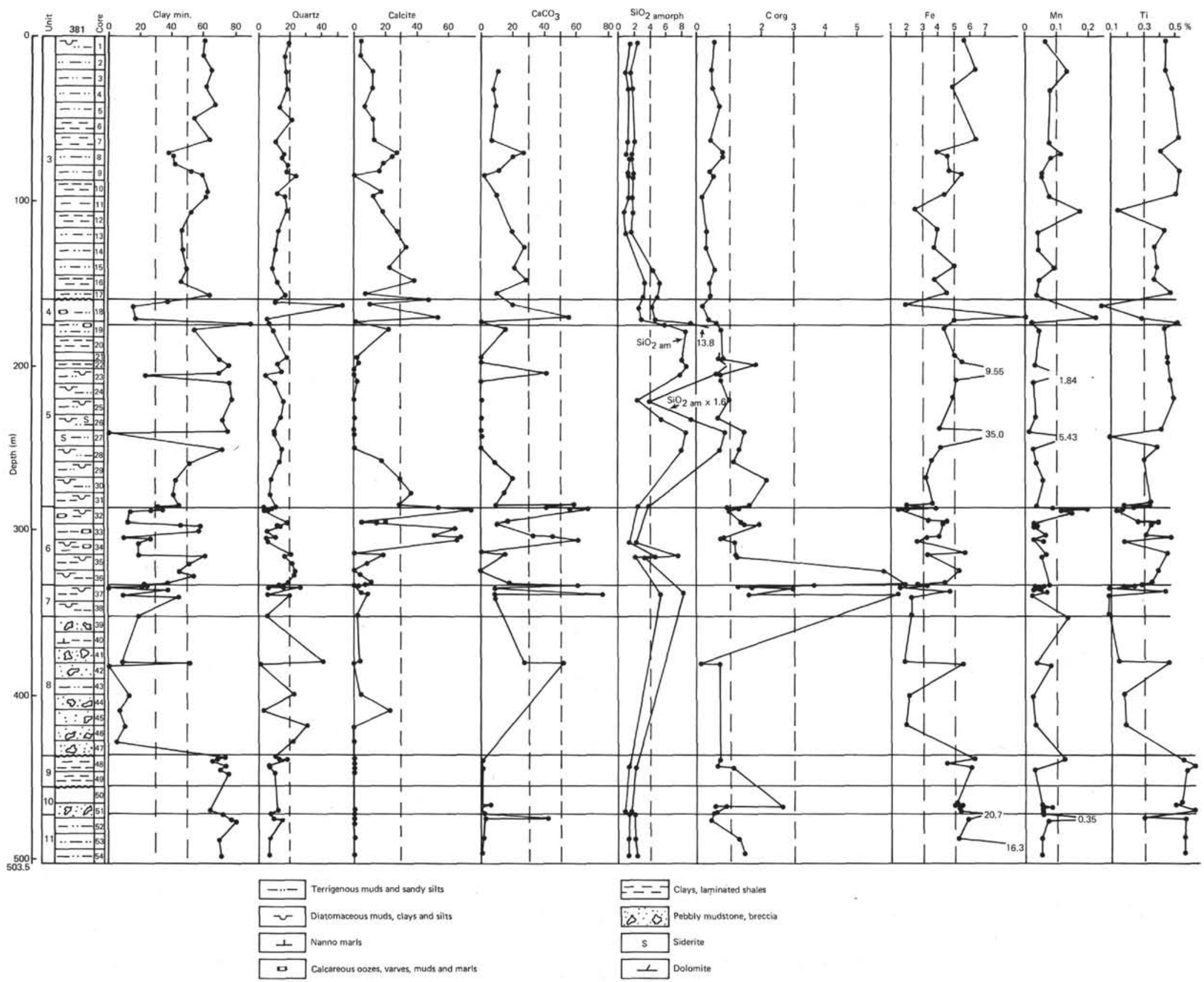

Figure 13. Lithologic composition of Site 381 sediments and distribution of main minerals and chemical macrocomponents. 

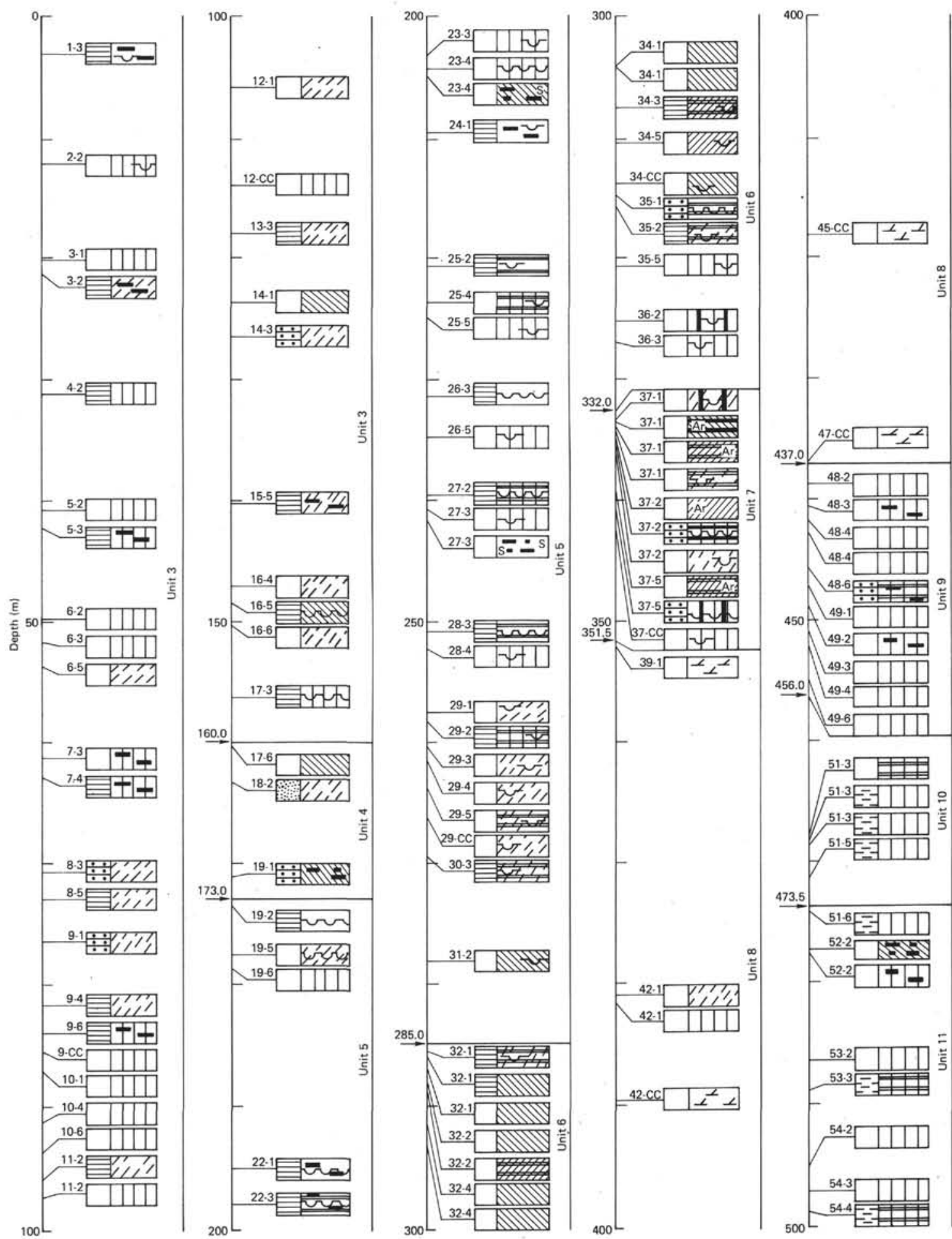<smiles>CC(C)(C)C1CC2C3C=CC(C3)C21</smiles>

Figure 14. Composition of Site 381 sediment samples. Designations as in Figure 4.

thin sections showed this rock to be characterized by fine-grained carbonate material with larger dolomite monocrystals (rhombohedra) present. A small admixture (5-15\%) of clastic material is present (quartz, $\mathrm{K}$-feldspars, and plagioclases make up a total slightly over $10 \%$, as determined by X-ray) as observed. These grains are corroded by carbonates. The rock has an admixture of finely-dispersed sulfides and fragments of organogenic carbonate. Pores are often filled with silica of opal-chalcedonic composition.

The studies of Samples 42, CC (Figure 19), 45, CC (Figure 20), 46, CC (Figure 21), 47, CC (Figure 22) in Unit 8 thin sections show lithified rudaceous and coarse-grained sediments (breccia, fine-pebbled conglomerates, sandstones) cemented by fine-grained dolomite (irregularly grained). The dolomite strongly 
TABLE 15

Chemical Composition of Bottom Sediments, Hole 381

\begin{tabular}{|c|c|c|c|c|c|c|c|c|c|c|c|c|c|c|c|c|c|}
\hline \multirow[b]{2}{*}{ Depth } & \multirow{2}{*}{$\begin{array}{c}\text { Sample } \\
\text { (Interval in } \mathrm{cm} \text { ) }\end{array}$} & \multicolumn{5}{|c|}{ Content (\%) } & \multicolumn{11}{|c|}{ Content $\left(10^{-4} \%\right)$} \\
\hline & & $\mathrm{CaCO}_{3}$ & $\mathrm{C}_{\text {org }}$ & $\mathrm{SiO}_{2}$ & $\mathrm{Fe}$ & $\mathrm{Mr}$ & $\mathrm{Ti}$ & $\mathrm{Ni}$ & Co & $\mathrm{Cr}$ & V & $\mathrm{Li}$ & $\mathrm{Rb}$ & Cs & $\mathrm{Pb}$ & $\mathrm{Sn}$ & $\mathrm{F}$ \\
\hline 3.00 & $1-3,0-20$ & 0.00 & 0.53 & 1.53 & 5.55 & 0.06 & 0.43 & - & - & - & - & 12 & 60 & 3.0 & 20 & 1.8 & - \\
\hline 12.24 & $2-2,124-144$ & 5.50 & - & - & - & - & - & 64 & 16 & 80 & 110 & - & - & - & - & - & 450 \\
\hline 20.20 & $3-1,120-145$ & 9.00 & - & - & - & - & - & - & - & - & - & - & - & - & - & - & - \\
\hline 21.05 & $3-2,155$ & 10.70 & 0.42 & 1.00 & 6.28 & 0.13 & 0.43 & 75 & 6 & 116 & - & 16 & 70 & 4.8 & 16 & 1.6 & - \\
\hline 31.15 & $4-2,115-140$ & 7.45 & 0.45 & 1.19 & 4.80 & 0.08 & 0.47 & - & - & - & - & 20 & 70 & 4.5 & 10 & 1.4 & - \\
\hline 40.70 & Sp2, $120-140$ & 9.50 & - & - & - & - & - & - & - & - & - & - & - & - & - & - & - \\
\hline 42.15 & $5-3,115-135$ & 8.88 & 0.67 & 1.36 & 5.86 & 0.07 & 0.47 & 80 & 18 & 75 & 160 & 20 & 90 & 5.0 & 13 & 1.6 & 430 \\
\hline 49.85 & $6-2,85-100$ & 6.50 & - & - & - & - & - & - & - & - & - & - & - & - & - & - & - \\
\hline 51.50 & $6-3,100-125$ & 9.26 & 0.24 & - & - & - & - & - & - & - & - & - & - & - & - & - & - \\
\hline 53.50 & $6-5,0-15$ & 12.00 & - & - & - & - & - & - & - & - & - & - & - & - & - & - & - \\
\hline 61.25 & $7-3,125-150$ & 6.50 & - & - & (6.3) & 0.051 & 0.40 & 68 & 19 & 41 & 150 & - & - & - & - & - & 540 \\
\hline 62.75 & $7-4,125-145$ & 6.26 & 0.40 & 1.21 & 5.30 & 0.07 & 0.51 & - & - & - & - & 30 & 120 & 7.0 & 12 & 1.0 & - \\
\hline 77.76 & $8-3,126-140$ & 26.86 & 0.79 & 1.14 & 3.89 & 0.11 & 0.40 & - & - & - & - & 22 & 90 & 6.6 & 10 & 1.0 & - \\
\hline 72.90 & $8-5,40-63$ & 20.77 & 0.79 & 1.24 & 4.50 & 0.08 & 0.43 & - & - & - & - & - & - & - & - & - & - \\
\hline 76.35 & $9-1,35-55$ & 10.26 & 0.39 & - & - & - & - & - & - & - & - & .- & - & - & - & - & - \\
\hline 81.85 & $9-4,135-150$ & 11.31 & 0.38 & 1.21 & 4.68 & 0.05 & 0.51 & - & - & - & - & 35 & 90 & 6.0 & 12 & 1.0 & - \\
\hline 84.20 & $9-6,70-90$ & 2.30 & 0.50 & 1.24 & 5.39 & 0.05 & 0.51 & - & - & - & - & 50 & 120 & 8.0 & 20 & 1.8 & - \\
\hline 85.50 & $9, \mathrm{CC}$ & 5.50 & - & - & - & - & - & - & - & - & - & - & - & - & - & - & - \\
\hline 86.75 & $10-1,125-150$ & 5.50 & - & - & - & - & - & 65 & 14 & 120 & 180 & - & - & - & - & - & 520 \\
\hline 91.15 & $10-4,115-150$ & 5.50 & 0.93 & _- & - & _- & _- & - & - & - & - & - & - & - & - & - & - \\
\hline 94.25 & $10-6,125-1$ & 7.00 & - & - & - & - & - & - & - & - & - & - & - & - & - & - & - \\
\hline 96.50 & $11-2,0-15$ & 10.42 & 0.16 & 1.25 & 4.38 & 0.07 & 0.49 & 80 & 18 & 77 & 160 & - & - & - & - & - & 370 \\
\hline 97.80 & $11-2,130-150$ & 7.50 & - & - & - & - & - & - & - & - & - & - & - & - & - & - & - \\
\hline 105.70 & $12-1,120-150$ & 18.50 & - & - & - & - & - & - & - & - & - & - & - & - & - & - & - \\
\hline 106.15 & $12-2,15-16$ & - & - & 0.85 & 2.46 & 0.17 & 0.14 & - & (4) & - & - & - & - & - & - & - & - \\
\hline 114.00 & $12, \mathrm{CC}$ & 10.00 & - & - & - & - & - & - & - & - & - & - & - & - & - & - & - \\
\hline 117.90 & $13-3,90-105$ & 19.74 & 0.29 & 1.05 & 3.85 & 0.04 & 0.42 & - & - & - & - & 35 & 90 & 7.0 & 8 & 1.0 & - \\
\hline 123.50 & $14-1,0-15$ & 34.00 & - & - & - & - & - & 56 & 13 & 48 & 110 & - & - & - & - & - & 320 \\
\hline 126.50 & $14-3,0-20$ & 27.94 & 0.29 & - & 3.65 & 0.04 & 0.36 & - & - & - & - & 40 & 80 & 7.0 & 10 & 1.0 & - \\
\hline 140.35 & $15-5,135-15$ & 21.48 & 0.52 & 2.73 & 5.06 & 0.09 & 0.38 & - & - & - & - & 50 & 120 & 8.0 & 13 & 1.3 & - \\
\hline 147.00 & $16-4,0-20$ & 10.26 & 0.27 & - & - & - & - & - & - & - & - & - & - & - & - & - & - \\
\hline 148.50 & $16-5 ; 0-20$ & 30.13 & 0.40 & 3.38 & 3.60 & 0.04 & 0.36 & 63 & 14 & 110 & 150 & 50 & 100 & 9.0 & 8 & 1.0 & 320 \\
\hline 150.00 & $16-6,0-20$ & 27.50 & - & - & - & - & - & - & (14) & - & - & - & - & - & - & - & - \\
\hline 156.35 & $17-3,135-150$ & 5.11 & 0.42 & 3.17 & 4.52 & 0.03 & 0.47 & - & - & - & - & 60 & 120 & 8.5 & 12 & 1.3 & - \\
\hline 160.80 & $17-6,130-150$ & 36.50 & - & - & - & - & - & 50 & 9 & 47 & 120 & - & - & - & - & - & 490 \\
\hline Average & & & 0.47 & 1.57 & 4.61 & 0.07 & 0.42 & 66 & 13 & 79 & 142 & 34 & 94 & 6.5 & 13 & 1.3 & 430 \\
\hline $168.0 \mathrm{C}$ & $18-2$ & 20.49 & 0.1 & 2.6 & 1.8 & 0.1 & 0.0 & - & - & - & - & 2 & 70 & 6.0 & 10 & 1.0 & - \\
\hline 171.00 & $19-1,0-15$ & 56.78 & 0.36 & 2.96 & 9.66 & 0.22 & 0.28 & 45 & (6) & 52 & - & 30 & 60 & 6.4 & 3 & - & 500 \\
\hline Average & & & 0.27 & 2.79 & 5.77 & 0.17 & 0.15 & 45 & 6 & 52 & - & 28 & 65 & 6.2 & 7 & 1.0 & 500 \\
\hline 173.42 & $19-2,92-102$ & 0.00 & 0.62 & 5.76 & 4.97 & 0.0 & 0.51 & 100 & 24 & 170 & 250 & 90 & 120 & 9.0 & 22 & 2.1 & 660 \\
\hline 177.86 & $19-5,86-88$ & 15.71 & 0.76 & $8.5 ?$ & 4.30 & 0.04 & 0.42 & - & - & - & - & - & - & - & - & - & - \\
\hline 178.57 & $19-6,7-24$ & 2.00 & - & - & - & - & - & - & - & - & - & - & - & - & - & - & - \\
\hline 195.40 & $22-1,40-55$ & 0.00 & 0.81 & 8.01 & 5.06 & 0.03 & 0.44 & - & - & - & - & 70 & 130 & 9.0 & 30 & 2.8 & - \\
\hline 198.40 & $22-3,40-55$ & 0.00 & 1.08 & 8.66 & 5.46 & 0.03 & 0.44 & 100 & 24 & 170 & 200 & 70 & 180 & 10.0 & 22 & 2.6 & 700 \\
\hline 204.10 & $23-4,10-25$ & 0.00 & 0.96 & - & - & - & - & - & - & - & - & - & - & - & - & - & - \\
\hline 204.92 & $23-4,92-94$ & 42.25 & 0.72 & 7.97 & 19.5 & 1.84 & $0.2 \varepsilon$ & - & - & - & - & - & - & - & - & - & - \\
\hline 209.48 & $24-1,48-63$ & 0.00 & 0.78 & - & 5.01 & 0.02 & 0.46 & - & (72) & - & - & 60 & 180 & 10.0 & 25 & 2.3 & - \\
\hline 220.40 & $25-2,40-55$ & 0.00 & 1.06 & 2.40 & 4.90 & 0.03 & 0.48 & 130 & 24 & 130 & 120 & 80 & 180 & 10.0 & 20 & 1.6 & 760 \\
\hline 223.50 & $25-4,50-65$ & 0.00 & 1.05 & - & - & - & - & - & - & - & - & - & - & - & - & - & - \\
\hline 224.7 & $26-4$ & 1.00 & - & - & - & - & - & - & - & - & - & - & - & - & - & - & - \\
\hline 231.40 & $26-3,40-50$ & 0.00 & 0.65 & 5.76 & 4.34 & 0.03 & 0.43 & 100 & 23 & 170 & 200 & 55 & 120 & 7.6 & 18 & 1.9 & 650 \\
\hline 234.55 & $26-5,55-70$ & 1.00 & - & - & - & - & - & - & - & - & - & - & - & - & - & - & - \\
\hline 239.50 & $27-2,50-65$ & 0.00 & 1.48 & 8.67 & 4.07 & 0.01 & 0.40 & - & (49) & - & - & 50 & 120 & 8.0 & 16 & 1.6 & - \\
\hline 240.50 & $27-3,0-15$ & 1.00 & - & - & - & - & - & - & - & - & - & - & - & - & - & - & - \\
\hline 241.27 & $27-3,77-79$ & - & - & - & 35.0 & 5.43 & 0.08 & - & 8 & - & - & - & - & - & - & - & - \\
\hline 250.70 & $28-3,70-85$ & 0.00 & 1.36 & 8.01 & 4.05 & 0.02 & 0.38 & 150 & 29 & 160 & 180 & 40 & 90 & 7.0 & 17 & 1.4 & 700 \\
\hline 252.05 & $28-4,55-70$ & 1. & - & - & - & - & - & - & - & - & - & - & - & - & - & - & - \\
\hline 257.34 & $29-1,84-100$ & 23.5 & - & - & _- & - & - & - & - & - & - & - & - & - & - & - & - \\
\hline 257.50 & $29-2,0-15$ & 8.58 & 1.13 & - & 3.52 & 0.03 & 0.30 & 80 & 18 & 83 & 140 & 25 & 60 & 5.0 & 20 & 2.0 & 550 \\
\hline 260.05 & $29-3,55-65$ & 19.01 & 0.90 & - & - & - & - & - & - & - & - & - & - & - & - & - & - \\
\hline 261.00 & $29-4,0-15$ & 20.50 & - & - & - & - & - & - & - & - & - & - & - & - & - & - & - \\
\hline 263.60 & $29-5,110-125$ & 17.26 & 1.20 & - & - & - & - & - & - & - & - & - & - & - & - & - & - \\
\hline 266.00 & $29, \mathrm{CC}$ & 25.50 & - & - & - & - & - & - & - & - & - & - & - & - & - & - & - \\
\hline 269.15 & $30-3,15-21$ & 20.99 & 1.70 & - & 3.15 & 0.05 & 0.31 & - & - & - & - & 28 & 120 & 6.5 & 12 & 1.6 & - \\
\hline 270.00 & $31-2,100-115$ & 31.50 & - & - & - & - & - & 31 & 9 & 62 & 120 & - & - & - & - & - & - \\
\hline Average & & & 1.02 & 7.08 & 7.95 & 0.58 & 0.38 & 99 & 19 & 135 & 173 & 57 & 130 & 8.2 & 20 & 1.9 & 670 \\
\hline
\end{tabular}


TABLE 15 - Continued

\begin{tabular}{|c|c|c|c|c|c|c|c|c|c|c|c|c|c|c|c|c|c|}
\hline \multirow[b]{2}{*}{ Depth } & \multirow[b]{2}{*}{$\begin{array}{c}\text { Sample } \\
\text { (Interval in cm) }\end{array}$} & \multicolumn{5}{|c|}{ Content (\%) } & \multicolumn{11}{|c|}{ Content $\left(10^{-4} \%\right)$} \\
\hline & & $\mathrm{CaCO}_{3}$ & $\mathrm{C}_{\text {org }}$ & $\mathrm{SiO}_{2}$ & $\mathrm{Fe}$ & $\mathrm{Mr}$ & $\mathrm{Ti}$ & $\mathrm{Ni}$ & Co & $\mathrm{Cr}$ & $\mathrm{V}$ & $\mathrm{Li}$ & $\mathrm{Rb}$ & Cs & $\mathrm{Pb}$ & $\mathrm{Sn}$ & F \\
\hline 285.31 & $32-1,31-44$ & 10.56 & 1.64 & - & 3.57 & 0.03 & 0.33 & - & - & - & - & 35 & 120 & 6.6 & 15 & 1.6 & - \\
\hline 285.81 & $32-1,81-94$ & 59.09 & 0.97 & 2.40 & 2.00 & 0.08 & 0.17 & - & - & - . & - & - & - & - & - & - & - \\
\hline 286.30 & $32-1,130-132$ & 42.44 & 0.82 & - & 3.80 & 0.19 & 0.23 & - & - & - & - & 20 & 60 & 5.0 & 3 & - & - \\
\hline 287.62 & $32-2,112-114$ & 56.86 & 0.98 & - & 1.99 & 0.11 & 0.17 & - & - & - & - & - & - & - & - & - & - \\
\hline 287.65 & $32-2,115-117$ & 68.54 & 1.01 & - & 1.41 & 0.14 & 0.12 & - & - & - & - & - & - & - & - & - & - \\
\hline 289.94 & $32-4,44-46$ & 34.00 & - & - & - & - & - & - & - & - & - & - & - & - & - & - & - \\
\hline 290.60 & $32-4,110-112$ & 50.78 & 0.48 & - & - & - & - & - & - & - & - & - & - & - & - & - & - \\
\hline 295.70 & $33-1,120-122$ & 1.85 & 1.36 & - & 2.21 & 0.02 & 0.25 & - & - & - & - & - & - & - & - & - & - \\
\hline 295.73 & $33-1,123-125$ & 14.10 & 1.49 & - & 3.40 & 0.03 & 0.38 & - & - & - & - & - & - & - & - & - & - \\
\hline 295.76 & $33-1,126-128$ & 10.94 & 1.08 & - & 3.27 & 0.02 & 0.33 & - & - & - & - & - & - & - & - & - & - \\
\hline 298.70 & $33-4,120-135$ & 40.03 & 1.05 & - & - & - & - & - & - & - & - & - & - & - & - & - & - \\
\hline 304.00 & $33, \mathrm{CC}$ & 6.00 & - & - & - & - & - & - & - & - & - & - & - & - & - & - & - \\
\hline 304.05 & $34-1,5-7$ & 33.74 & 0.86 & - & 3.00 & 0.06 & 0.30 & - & - & - & - & - & - & - & - & - & - \\
\hline 304.08 & $34-1,8-10$ & 46.54 & 0.77 & - & 2.15 & 0.04 & 0.46 & - & - & - & - & - & - & - & - & - & - \\
\hline 307.10 & $34-3,10-20$ & 61.90 & 1.19 & 1.40 & 1.58 & 0.05 & 0.16 & 18 & 5 & 25 & 65 & - & - & - & - & - & 220 \\
\hline 310.23 & $34-5,23-33$ & 72.05 & 0.78 & - & - & - & - & - & (3) & - & - & - & - & - & - & - & - \\
\hline 313.50 & $34, \mathrm{CC}$ & 30.50 & - & - & - & - & - & - & - & - & - & - & - & - & - & - & - \\
\hline 314.60 & $35-1,110-120$ & 0.00 & 1.22 & 4.70 & 4.70 & 0.06 & 0.45 & - & - & - & - & 50 & 120 & 8.0 & 13 & 1.8 & - \\
\hline 315.60 & $35-2,60-70$ & 17.24 & 1.24 & 2.22 & 4.18 & 0.05 & 0.43 & 80 & 23 & 97 & 180 & 50 & 180 & 9.0 & 14 & 1.6 & 600 \\
\hline 324.94 & $36-2,44-46$ & 0.00 & 5.88 & - & 4.29 & 0.06 & 0.38 & - & (26) & - & - & - & - & - & - & - & - \\
\hline 326.70 & $36-3,70-84$ & 2.00 & - & - & - & - & - & - & - & - & - & - & - & - & - & - & - \\
\hline \multicolumn{2}{|l|}{ Average } & & 1.34 & 2.68 & 2.97 & 0.07 & 0.30 & 49 & 14 & 61 & 122 & 39 & 120 & 7.0 & 11 & 1.7 & 410 \\
\hline 333.26 & $37-1,76-78$ & 18.90 & 6.56 & - & 3.49 & 0.07 & 0.34 & - & - & - & - & - & - & - & - & - & - \\
\hline 333.40 & $37-1,90-92$ & 37.28 & 3.88 & - & 2.42 & 0.06 & 0.25 & - & (6) & - & - & - & - & - & - & - & - \\
\hline 333.84 & $37-1,134-136$ & 61.99 & 1.29 & - & 1.56 & 0.03 & 0.17 & - & (8) & - & - & - & - & - & - & - & - \\
\hline 333.96 & $37-1,146-148$ & 29.66 & 1.70 & - & 2.28 & 0.05 & 0.22 & - & - & - & - & - & - & - & - & - & - \\
\hline 334.12 & $37-2,12-14$ & 81.84 & 0.58 & - & 0.65 & 0.02 & 0.07 & - & - & - & - & - & - & - & - & - & - \\
\hline 334.30 & $37-2,30-40$ & 9.36 & 3.07 & 5.17 & 3.78 & 0.06 & 0.43 & 80 & 22 & 87 & 160 & 40 & 150 & 8.0 & 15 & 1.0 & 600 \\
\hline 335.05 & $37-2,105-107$ & 18.26 & 0.15 & - & - & - & - & - & - & - & - & - & - & - & - & - & - \\
\hline 339.06 & $37-5,56-58$ & 78.17 & 1.63 & - & 1.25 & 0.02 & 0.07 & - & - & - & - & - & - & - & - & - & - \\
\hline 339.48 & $37-5,98-110$ & 8.69 & 6.27 & 3.60 & 3.80 & 0.07 & 0.43 & - & - & - & - & 45 & 200 & 10.0 & 20 & 1.7 & - \\
\hline 342.00 & $37, \mathrm{CC}$ & 4.00 & - & - & - & - & - & - & - & - & - & - & - & - & - & - & - \\
\hline \multicolumn{2}{|l|}{ Average } & & 2.86 & 4.39 & 2.40 & 0.05 & 0.25 & 80 & 12 & 87 & 160 & 43 & 175 & 9.0 & 18 & 1.4 & 600 \\
\hline 351.92 & $39-1,42-44$ & - & - & - & 1.39 & 0.13 & 0.08 & - & - & - & - & - & - & - & - & - & - \\
\hline 380.90 & $42-1,90-100$ & 28.76 & 0.12 & - & 0.98 & 0.03 & 0.13 & - & - & - & - & - & - & - & - & - & - \\
\hline 381.40 & $42-1,140-145$ & 5.30 & 0.69 & - & 4.56 & 0.07 & 0.46 & - & - & - & - & - & - & - & - & - & - \\
\hline 408.50 & 44, CC & - & - & - & 1.21 & 0.04 & 0.17 & - & - & - & - & - & - & - & - & - & - \\
\hline 427.50 & $46, \mathrm{CC}$ & - & - & - & 1.07 & 0.03 & 0.18 & - & - & - & - & - & - & - & - & - & - \\
\hline Average & & - & 0.41 & - & 1.84 & 0.06 & 0.20 & - & - & - & - & - & - & - & - & - & - \\
\hline 435.55 & $48-2,5-17$ & 1.00 & - & - & - & - & - & 110 & 28 & 150 & 150 & - & - & - & - & - & 640 \\
\hline 440.55 & $48-3,55-57$ & 1.21 & 0.71 & - & 5.32 & 0.12 & 0.53 & - & - & - & - & - & - & - & - & - & - \\
\hline 441.50 & $48-4,0-10$ & 0.00 & 0.84 & - & - & - & - & - & - & - & - & - & - & - & - & - & - \\
\hline 442.38 & $48-4,88-90$ & 1.00 & - & - & - & - & - & - & (12) & - & - & - & - & - & - & - & - \\
\hline 444.20 & $48-5,120-130$ & 0.00 & 0.85 & 1.26 & 3.56 & 0.06 & 0.60 & - & - & - & - & 70 & 200 & 12.0 & 25 & 2.5 & - \\
\hline 445.60 & $48-5,110-120$ & 0.77 & 1.12 & 1.24 & 5.10 & 0.05 & 0.55 & - & - & - & - & 60 & 150 & 9.0 & 24 & 2.3 & - \\
\hline 446.70 & $49-1,20-34$ & 1.00 & - & - & - & - & - & 160 & 30 & 180 & 180 & - & - & - & - & - & 330 \\
\hline 450.58 & $49-3,108-120$ & 1.00 & - & - & - & - & - & - & - & - & - & - & - & - & - & - & - \\
\hline 451.50 & $49-4,50-65$ & 0.75 & 0.69 & - & - & - & - & - & - & - & - & - & - & - & - & - & - \\
\hline 454.30 & $49-6,30-42$ & 1.00 & - & - & - & - & - & - & - & - & - & - & - & - & - & - & - \\
\hline Average & & & 0.84 & 1.25 & 4.69 & 0.08 & 0.56 & 135 & 23 & 165 & 165 & 65 & 175 & 11.0 & 25 & 2.3 & 485 \\
\hline 468.56 & $51-3,6-8$ & 0.89 & 2.71 & - & 4.21 & 0.0 & 0.52 & - & - & - & - & - & - & - & - & - & - \\
\hline 468.64 & $51-3,14-16$ & 6.97 & 0.58 & - & 4.63 & 0. & 0.49 & - & - & - & - & - & - & - & - & - & - \\
\hline 468.80 & $51-3,30-32$ & 1.50 & 0.94 & - & 4.16 & 0.05 & 0.52 & - & - & - & - & - & - & - & - & - & - \\
\hline 470.50 & $51-5,0-12$ & 1.00 & 0.62 & 0.96 & 4.38 & 0.0 & 0.55 & - & - & - & - & 60 & 200 & 12.0 & 30 & 2.5 & - \\
\hline 473.73 & $51-6,73-88$ & 1.75 & 0.55 & 1.13 & 4.47 & 0.05 & 0.60 & 130 & 22 & 130 & 140 & 56 & 180 & 10.0 & 25 & 2.2 & 580 \\
\hline Average & & & 1.08 & 1.05 & 4.37 & 0.06 & 0.59 & 130 & 22 & 130 & 140 & 58 & 190 & 11.0 & 28 & 2.4 & 580 \\
\hline 477.12 & $52-2,62-63$ & 43.96 & 0.47 & - & 20.70 & 0.35 & 0.31 & - & - & - & - & - & - & - & - & - & - \\
\hline 477.15 & $52-2,65-67$ & 3.24 & 0.43 & - & 5.01 & 0.07 & 0.55 & - & (21) & - & - & - & - & - & - & - & - \\
\hline 486.19 & $53-2,19-29$ & 6.50 & - & - & - & - & - & - & - & - & - & - & - & - & - & - & - \\
\hline 488.75 & $53-2,125-135$ & 1.80 & 1.35 & 1.17 & 4.38 & 0.05 & 0.55 & - & - & - & - & 45 & 180 & 10.0 & 22 & 2.4 & - \\
\hline 499.55 & $54-2,5-16$ & 1.00 & - & - & - & - & - & 180 & 27 & 210 & 160 & - & - & - & - & - & 550 \\
\hline 497.26 & $54-3,26-38$ & 2.50 & 0.87 & - & - & - & - & - & - & - & - & - & - & - & - & - & - \\
\hline 498.53 & $54-4,3-13$ & 1.55 & 1.49 & 1.38 & 4.38 & 0.05 & 0.55 & - & - & - & - & 45 & 180 & 10.0 & 22 & 1.8 & - \\
\hline 502.73 & $54-6,123-125$ & 1.00 & - & - & 3.35 & 0.04 & 0.38 & 100 & (21) & 125 & - & - & - & - & - & - & - \\
\hline Average & & & 0.92 & 1.28 & 7.56 & 0.11 & 0.47 & 140 & 23 & 168 & 160 & 45 & 180 & 10.0 & 22 & 2.1 & 550 \\
\hline Average & for hole & & 1.12 & 3.14 & 4.64 & 0.17 & 0.36 & 62 & 20 & 110 & 147 & 44 & 124 & 8.0 & 16 & 1.7 & 526 \\
\hline
\end{tabular}


TABLE 16

Average Contents of Components and Elements in the Different Units of Hole 381

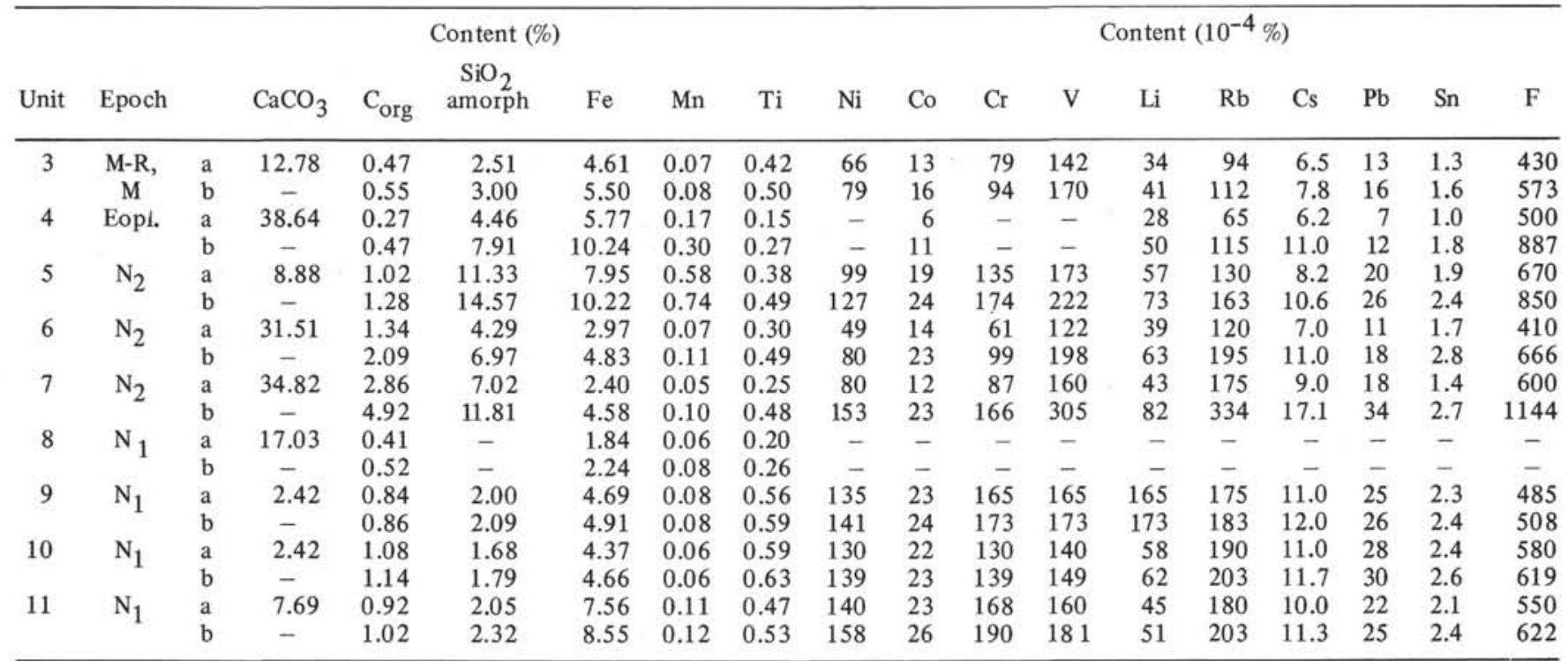

Note: $\mathrm{a}=\mathrm{a}$ natural dry sediment; $\mathrm{b}=$ on a carbonate, organic $\left(\mathrm{C}_{\text {org }} \times 2\right)$ matter free basis.

TABLE 17

Average Contents of $\mathrm{CaCO}_{3}, \mathrm{SiO}_{2}$ amorph, and $\mathrm{C}_{\text {org }}$ in Sediments of the Black Sea

\begin{tabular}{lccccccc}
\hline & \multicolumn{2}{c}{$\mathrm{CaCO}_{3}$} & \multicolumn{3}{c}{$\mathrm{SiO}_{2 \text { amorph }} \times 1.6$} & \multicolumn{2}{c}{$\mathrm{C}_{\text {org }}$} \\
Hole & 1 & 2 & 1 & 2 & 1 & 2 \\
\hline $379 \mathrm{~A}$ & a & $0.00-73.80$ & 17.63 & $1.65-9.25$ & - & $0.27-6.00$ & 0.69 \\
& b & - & - & - & - & $0.40-6.89$ & - \\
380 & a & $4.00-24.92$ & 10.35 & $1.44-13.15$ & 2.59 & $0.20-1.94$ & 0.63 \\
& b & - & - & $1.65-14.97$ & 2.93 & $0.30-2.46$ & 0.72 \\
$380 \mathrm{~A}$ & a & $0.00-63.50$ & 23.68 & $1.02-18.94$ & 3.82 & $0.08-3.18$ & 0.74 \\
& b & - & - & $1.75-19.70$ & 5.10 & $0.17-3.73$ & 1.02 \\
381 & a & $0.00-81.84$ & 17.17 & $1.36-13.87$ & 5.02 & $0.12-6.56$ & 1.12 \\
& b & - & - & $1.58-22.64$ & 6.23 & $0.18-7.93$ & 1.44 \\
All holes & a & $0.00-81.84$ & & $1.02-18.94$ & & $0.08-6.56$ & \\
& b & - & & $1.58-22.64$ & & $0.17-7.93$ & \\
\hline
\end{tabular}

Note: $\mathrm{a}=$ natural sediment $; \mathrm{b}=$ on a carbonate-free basis; $1=$ range, $2=$ average.

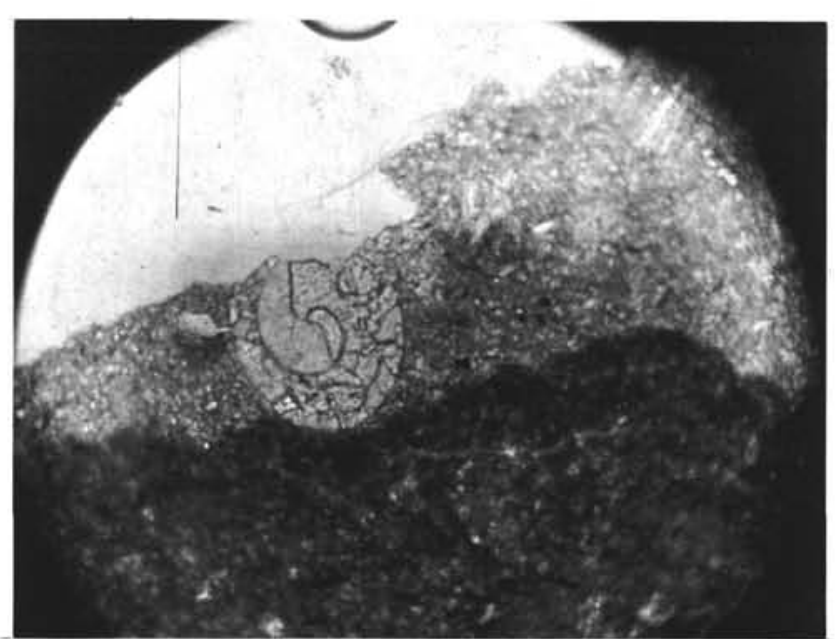

Figure 15. Calcareous rock partly replaced by ankerite with relictorganogenic structure. Site 380A, Sample 59-1-117$118 \mathrm{~cm}$, crossed nicols, $\times 20$.

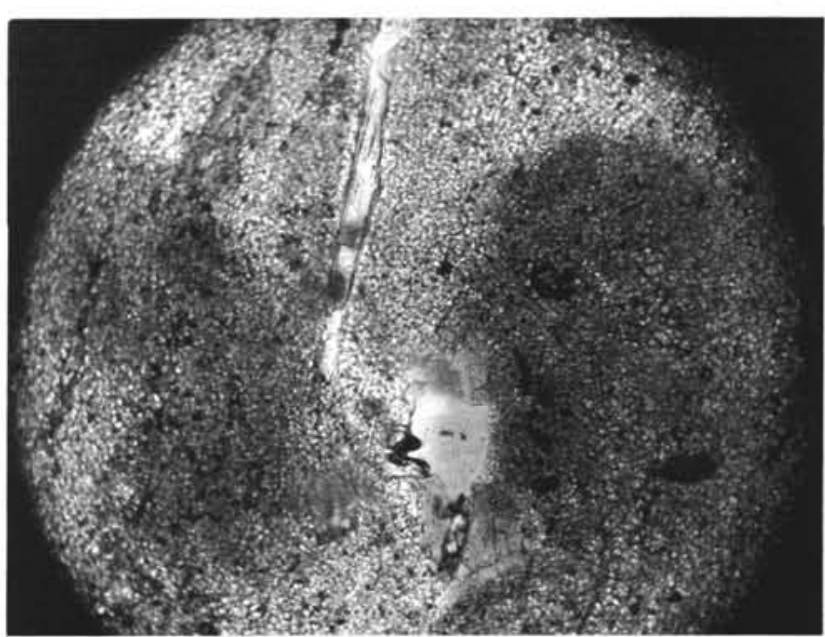

Figure 16. Clayey dolomite rock. Site 380A, Sample 73-1, $58-60 \mathrm{~cm}$, crossed nicols, $\times 20$.

corrodes the clastic material present (quartz, feldspars, plagioclase, rock fragments). This is shown in the photomicrographs (Figures 19-22).

The lower sediments (Units 9-11) are clay muds with rather low $\mathrm{CaCO}_{3}$ contents (below 1-5\%), either enriched or impoverished in organic matter. Calcite and siderite occur as traces. A relatively high $\mathrm{CaCO}_{3}$ content $(43.96 \%)$ occurs in a single layer $(52-2,62-63$ $\mathrm{cm})$, the mineral composition of which was not determined.

From the above-mentioned data, the carbonate distribution characteristics in late Cenozoic sediments are as follows:

1) $\mathrm{CaCO}_{3}$ contents in most of the section are lower (10-30\%) than in upper Quaternary deposits in the same areas. An increase in carbonates is seen only in the 


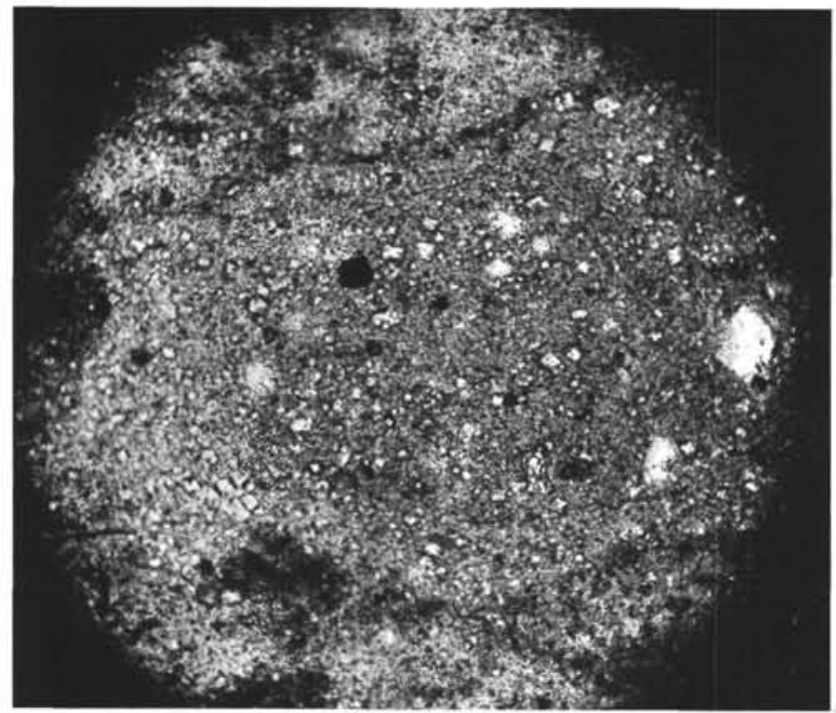

Figure 17. Calcareous-clay rock with siderite, dolomitemonocrystal. Site 381, Sample 12-2, 15-16 cm, crossed nicols $\times 20$.

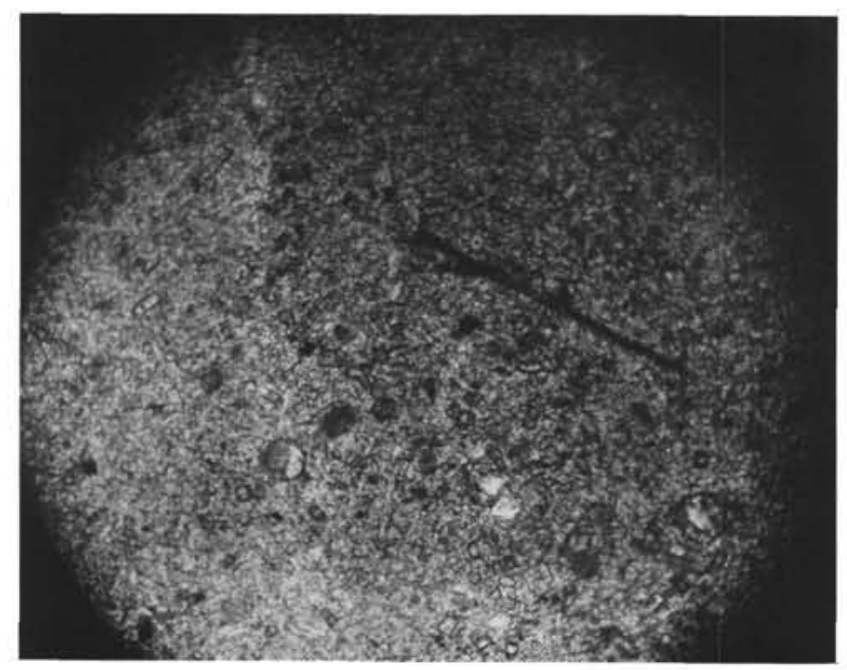

Figure 18. Siderite rock with relict-organogenic structure formed as a result of diatom mud replacement. Site 381, Sample 27-3, 77-79 cm, parallel nicols, $\times 20$.

lowermost sections. At Site 379, the carbonate mineral composition is almost invariably calcite, with sporadic dolomite and rare aragonite. The mineralogy at Site 380 is completely different; the lower part, in contrast to the upper, contains siderite (sporadic) in addition to calcite and dolomite. This is characteristic of early Pleistocene sediments of Hole 380A and of PleistoceneEopleistocene deposits of Site 381 as well. Although $\mathrm{CaCO}_{3}$ contents in Quaternary sediments of Sites 379 and 380 are almost similar, there is an evident difference in mineral composition, with dolomite ubiquitous at Site 380 (siderite is sporadic in the lower part of the section). This latter feature is also characteristic of the lower Pleistocene sediments of Site 381.

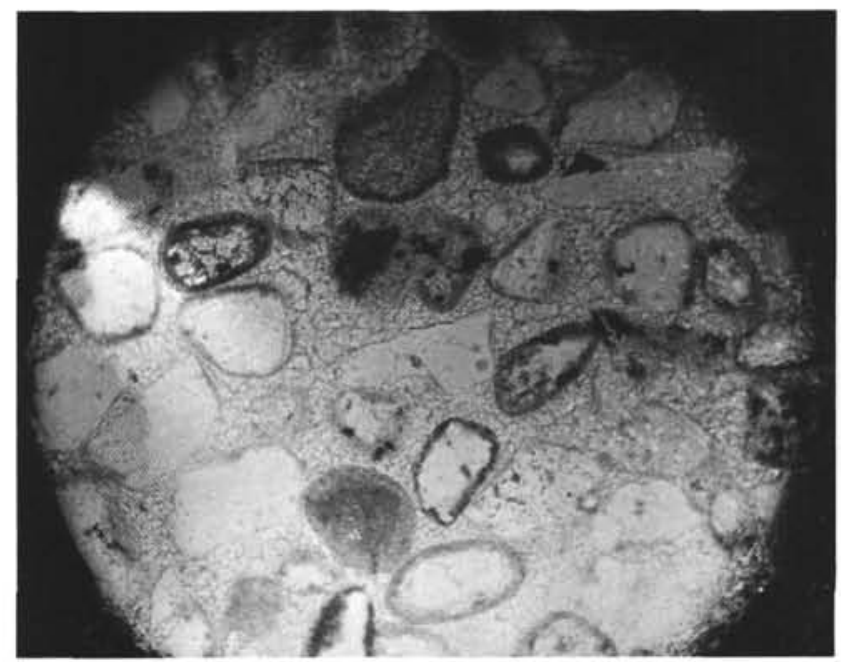

Figure 19. Sandy part of fine pebble conglomerate cemented by calcite-dolomite corrosive cement. Site 381, Sample 44, CC, parallel nicols, $\times 20$.

2) Pliocene-Miocene deposits of Hole $380 \mathrm{~A}$ and Site 381 differ markedly from Quaternary ones both in $\mathrm{CaCO}_{3}$ content, its distribution pattern, and in carbonate mineral composition. Here sediment layers containing rather high (to $81 \%$ ) or low (almost zero) $\mathrm{CaCO}_{3}$ contents alternate repeatedly; this phenomenon is not observed in the Quaternary sediments. In addition, beds consisting entirely of clayey dolomite, dolomite with a calcite admixture, siderite, and enriched in or totally composed of aragonite are found.

The Miocene and Pliocene deposits are characterized by a correspondence of definite series of carbonates to definite types of sediments. Mineral assemblages with aragonite show a clear association with muds enriched in diatomaceous silica containing both low and high amounts of organic matter. The complex variability is to a certain extent dependent on Corg contents. Where they are much lower than $1 \%$, aragonite occurs in maximum amounts (Site 381, 37-2, 12-14 cm); at higher Corg contents in diatomaceous muds, aragonite is accompanied by calcite and dolomite.

\section{Carbonate Genesis Features}

Microscopic studies revealed a genetic heterogeneity of the carbonates:

Calcite: In Quaternary deep-water sediments, calcite is mainly clastic, evidenced by its constant occurrence and relatively regular distribution. Only in Interglacial units is biogenic calcite also of considerable importance, especially at Site 379 where layers of solid coccoliths are found. As in the Holocene sediments, calcareous nannoplankton material in the Interglacial series occurs in muds of varying carbonate content.

A part of the nonbiogenic calcite is likely to be of diagenetic origin. The percentage of diagenetic calcite is evidently considerable in sediments of Holes $380,380 \mathrm{~A}$, and 381 , where calcite is accompanied by large quantities of siderite and dolomite. The possibility of carbonate precipitation from interstitial waters in the 


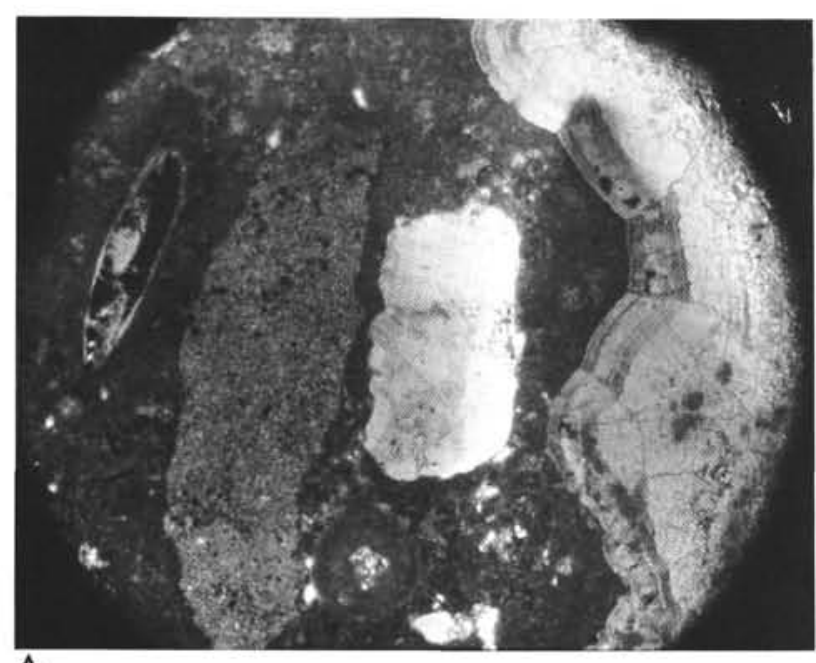

A.

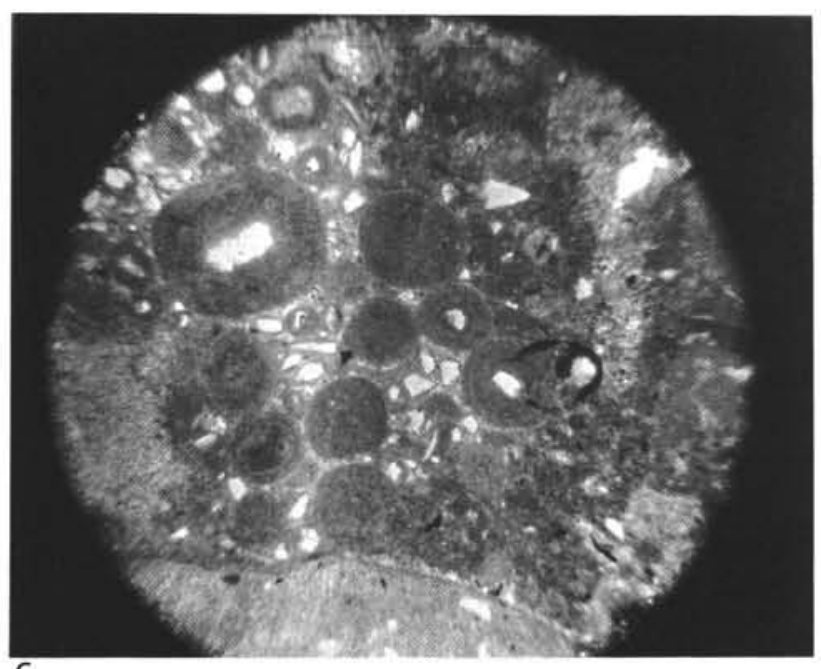

c.

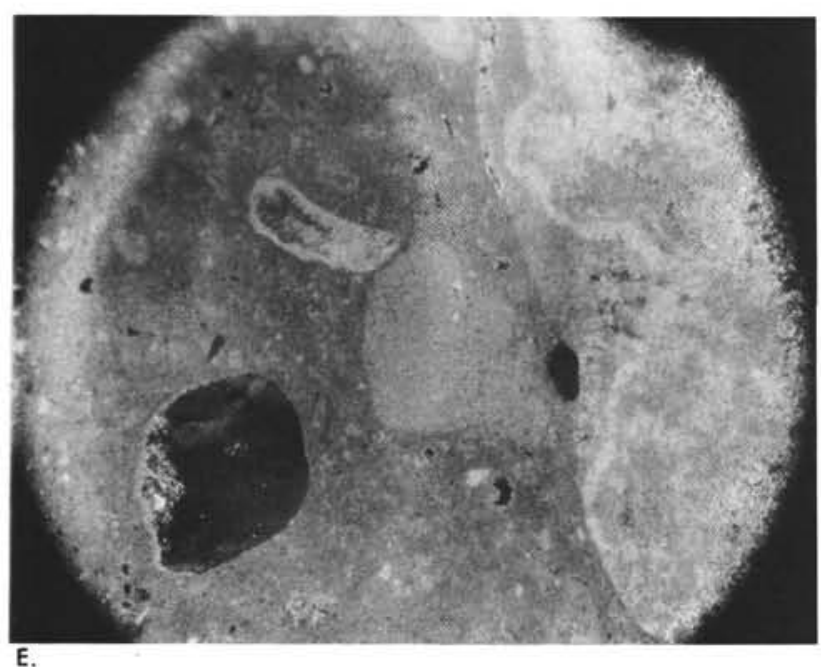

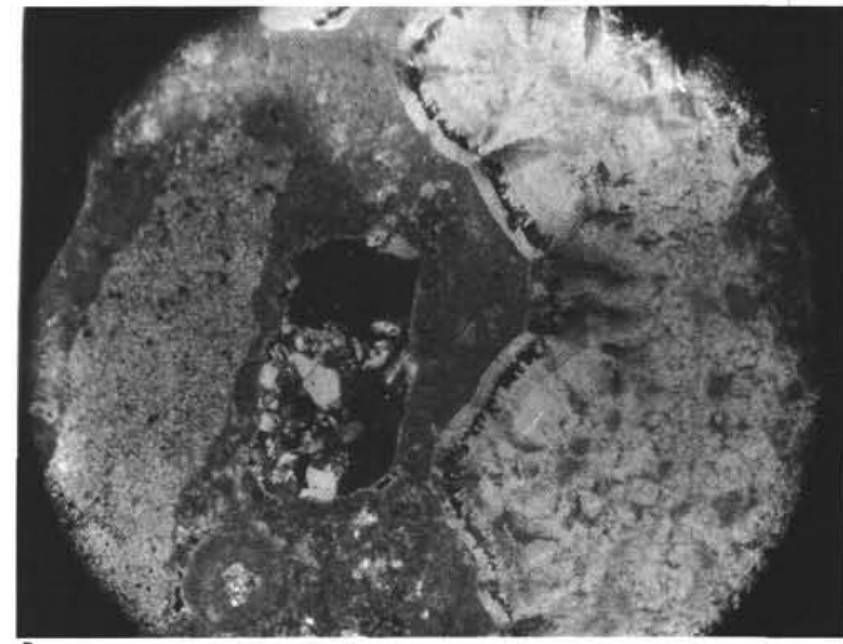

B.

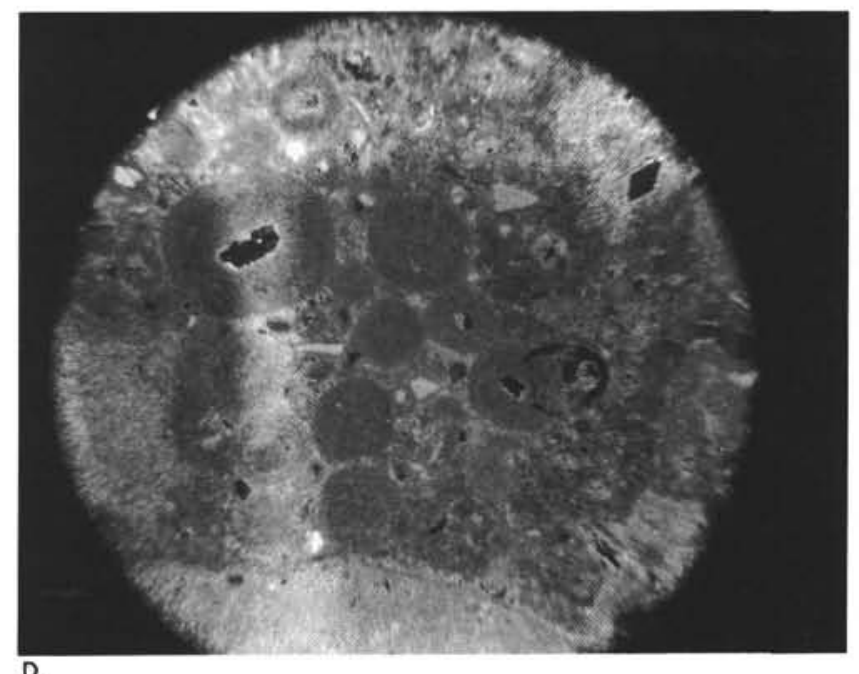

D.

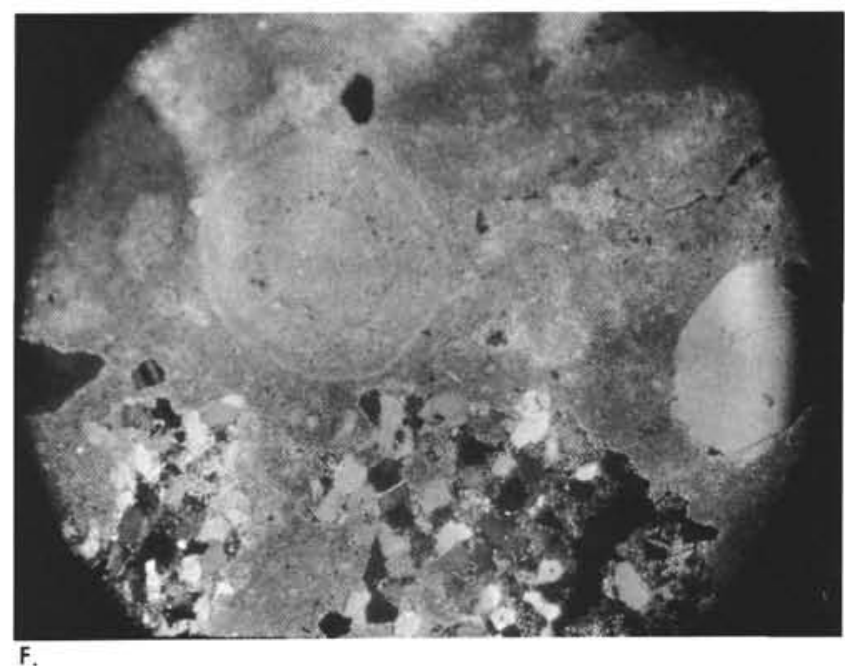

Figure 20. Conglomerate-breccia consisting of dolomitized rock fragments, quartzites, sandstones, aleuralites, oolites, and other formations by microgranular dolomite. Dolomite corrosive cement. (A) Site 381, Sample 45, CC, parallel nicols 11, X9. (B) Site 381, Sample 45, CC, crossed nicols, X9. (C) Site 381, Sample 45, CC, parallel nicols, X9. (D) Site 381, Sample 45, CC, crossed nicols, X9. (E) Site 381, Sample 45, CC, crossed nicols, X9. (F) Site 381, Sample 45, CC, crossed nicols, $\times 9$. 


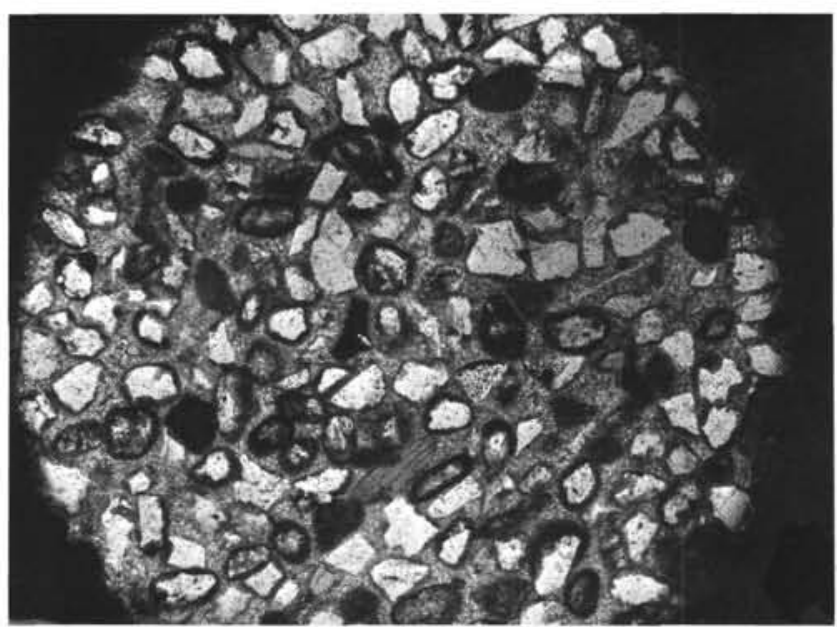

Figure 21. Quartz-feldspar sandstone cemented by dolomite corrosive cement. Site 381, Sample 46, CC, parallel nicols, $\times 9$.

upper Quaternary Black Sea sediments was suggested by Shishkina $(1959,1972)$. This process may also be considered for the rest of the section, especially since pure single transparent carbonate crystals, irregularly or rhombohedrally shaped, are often seen under the microscope.

Aragonite: In Quaternary sediments, aragonite occurs in the upper part of the section. At Site 379, aragonite occurs with dolomite in muddy beds (from the last Interglacial series, Unit 5, R-W) with a high organic matter content. At Site 380, a number of beds containing aragonite occur in deposits of the last Glacial stage (Unit B-1-c, W). Compared to surrounding sediments, these muds are poor in organic matter, but relatively enriched in diatoms. Here aragonite occurs with equal amounts of dolomite and calcite.

Aragonite is present in several Neogene units, mainly occurring in diatomaceous muds in combination either with calcite, or calcite and dolomite.

The combination of aragonite and dolomite suggests a diagenetic origin. Being a metastable mineral, aragonite may survive after precipitation from mud solutions only when pore waters are oversaturated with calcium carbonate. This case is observed at Holes 380, $380 \mathrm{~A}, 381$, and in the upper parts of Site 379 (see section on interstitial water geochemistry).

Dolomite: In the Quaternary sediments of Site 379, dolomite is either absent or present only in small quantity. At Site 380 , it is abundant, occurring in quantities equal to or exceeding calcite in a number of interbeds. Dolomite content is usually considerably lower than calcite in lower Pleistocene sediments (Site 381 ) and in Eopleistocene (Holes 380A, 381) where often it is absent. The occurrence of dolomite in combination with both calcite and siderite in the lower part of the Quaternary section is characteristic, but a constant correlation between dolomite and siderite is not observed.
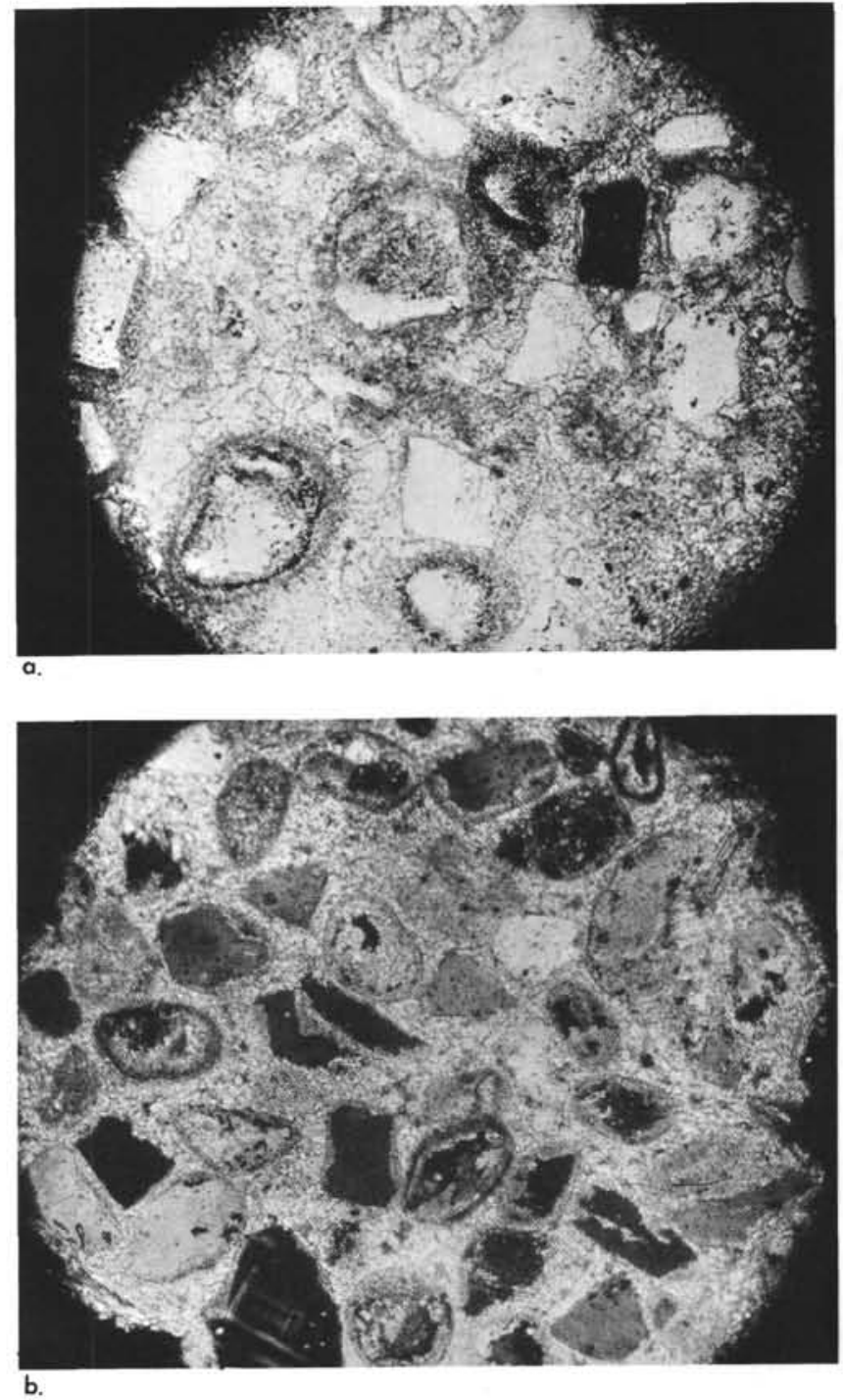

Figure 22. Quartz-feldspar sandstone cemented by dolomite corrosive cement. (a) Site 381, Sample 47, CC, crossed nicols, X9. (b) Site 381, Sample 47, CC, parallel nicols, $\times 9$.

Dolomite is less frequent in Neogene than in Quaternary sediments, forming generally isolated interbeds without admixtures of other carbonate minerals.

The pattern of dolomite distribution in the section and its patterns of occurrence with other minerals suggest a diagenetic origin for most of the dolomite amount; this is confirmed by microscopic observations of thin sections and smear slides.

Siderite: Siderite is not found in the Quaternary sediment section of Site 379 nor in the upper part of Site 380 . It is found sporadically in the lower half of the section as a minor admixture to other minerals (dolomite, calcite), and generally increases down section. A steady increase of siderite content in Pliocene sediments is characteristic; it is found occurring either with calcite or with dolomite and calcite, very rarely 
with dolomite alone. Beds consisting totally of siderite also occur (Figure 23).

No doubt exists as to the diagenetic origin of siderite, which is evidenced by relict structures clearly showing replacement of diatomaceous muds.

According to Hunt (this volume), $\mathrm{Ca}^{2+}$ and $\mathrm{Mg}^{2+}$ concentrations in the late Cenozoic sediment section (Holes 380 and 380A) are subject to specific variations: they are highest in the upper part of the section and show minimum values in the middle (with crossing curves). In the lower part, fluctuations of opposite sign occur.

It was already noted on board ship that siderite appears in the sediments when $\mathrm{Mg}^{2+}$ and $\mathrm{Ca}^{2+}$ concentrations (Cores 1-56 in Hole 380A, 330-850 m) are sharply decreased in the interstitial waters, with contents of both cations being almost level. Interstitial water is considerably enriched in $\mathrm{Mg}^{2+}$ and, to a lesser extent, in $\mathrm{Ca}^{2+}$ above this level $(0-330 \mathrm{~m}$, Site 380). As noted above, calcite and, only sporadically dolomite and siderite, are distributed in this interval. Below this, the $\mathrm{Ca}^{2+}$ curve drops sharply whereas that of $\mathrm{Mg}^{2+}$ is increased. Here, calcite and siderite are found in small quantities, and dolomite is completely absent.

Thus, a certain relationship exists between characteristic variations of carbonate mineral composition and those of $\mathrm{Ca}^{2+}$ and $\mathrm{Mg}^{2+}$ concentrations. This is another line of evidence in favor of diagenetic formation of the major carbonates in Holes 380/380A (and probably at Site 381 as well) and also explains why siderite is present only in the middle

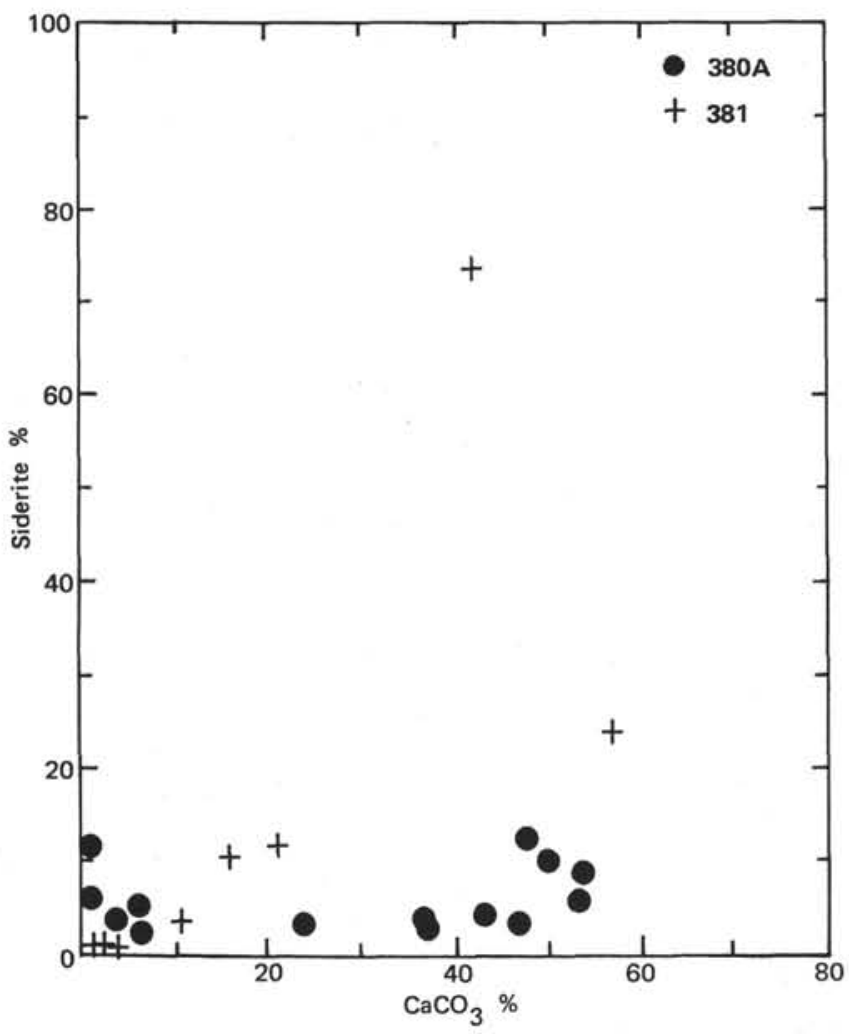

Figure 23. Siderite distribution in Hole $380 \mathrm{~A}$ and Site 381 sediments as a function of $\mathrm{CaCO}_{3}$ contents. and not the upper part of the section. High exchangeable $\mathrm{Ca}^{2+}$ and $\mathrm{MG}^{2+}$ concentrations allowed bonding of the bulk of hydrocarbonates into calcite and dolomite molecules, while a lack of $\mathrm{Ca}^{2+}$ and $\mathrm{Mg}^{2+}$ in the middle resulted in bonding of a part of the hydrocarbonates as the ferruginous carbonate molecule. Formation of a pure siderite bed may only be possible with negligibly low exchangeable $\mathrm{Ca}^{2+}$ and $\mathrm{Mg}^{2+}$ content, i.e., where the sediment's biogenic component consists mainly of silica, thus explaining why diatomaceous muds were the facies replaced by siderite.

Diagenetic processes of formation for the various carbonate minerals is not yet clear. It is known (Shishkina, 1972) that changes in pore water composition began during early diagenesis: anion and cation compositions change, resulting in formation of pore waters impoverished in sulfate and conditions suitable for precipitation of carbonate from solutions. Shishkina's description (1972, p. 121-122) of this process is cited: "In interstitial waters, a sulfate reduction is followed by a $\mathrm{SO}_{4}$-ion replacement $\mathrm{HCO}_{3}$ and $\mathrm{CO}_{3}$ ions, and an increase in $\mathrm{pH}$. With accumulation of these ions and a shift of dissociation towards carbonate ion formation, $\mathrm{CaCO}_{3}$ is deposited from the interstitial water, resulting in decrease of $\mathrm{Ca}^{2+}$ concentration at this stage in the upper 2-3 meters of sediments. Ions, including $\mathrm{Na}^{2+}$ and $\mathrm{Mg}^{2+}$, diffuse from the interstitial waters of the upper sediment beds with higher ion concentration to lower beds resulting in $\mathrm{Ca}^{2+}$ displacement from the absorbing complex to the interstitial water, where it accumulates. Exchangeable $\mathrm{Ca}^{2+}$ decreases the $\mathrm{CaCO}_{3}$ solubility (common ion effect), thus providing further $\mathrm{CaCO}_{3}$ deposition and alkaline reserve decrease."

The presence of chemogenetic-diagenetic carbonates in Pleistocene and older sediments suggests that pore water diagenesis continues during later diagenetic stages as well. From our study of these materials, it is concluded that chemogenetic-diagenetic calcite is formed in $\mathrm{CaCO}_{3}$-enriched sediments (coccoliths, clastic calcite), i.e., in the presence of surplus $\mathrm{Ca}$. When $\mathrm{Ca}$ is lacking, surplus $\mathrm{CO}_{2}$ is bonded in mixed calcite and dolomite and ferruginous carbonates (less often ferro-manganous carbonate). In cases of $\mathrm{Ca}$ absence, siderite concretions may form, as shown by early studies of concretions (Vital, 1959, p. 235). The $\mathrm{CO}_{2}$ required for ferruginous carbonate formation might result from bacterial activity during the decomposition of organic matter.

The process of chemogenetic-diagenetic carbonate formation here proceeded so far as to begin to replace various clastic minerals, beginning with the feldspars (Figures 20-22).

Many processes of dolomitization exist, according to Friedman and Sanders (1970). Dolomitization may be either selective or general. In the Black Sea it is selective, for only discrete beds are considerably enriched in dolomite. Dolomitization mainly occurs at increased $\mathrm{Mg} / \mathrm{Ca}$ ratio, with calcite and aragonite converted to dolomite by the following reaction (Berner, 1965): 


$$
\mathrm{Mg}_{\text {dis }}^{2+}=2 \mathrm{CaCO}_{3} \rightleftharpoons \mathrm{CaMg}\left(\mathrm{CO}_{3}\right)_{2}=\mathrm{Ca}_{\text {dis }}^{2+}
$$

Simultaneously, the calcium ion might be bonded as gypsum (gypsum is also found in the Black Sea sediments).

\section{Amorphous Silica}

Recent deep-water sediments contain small amounts of amorphous silica $(<1-5 \%)$. Thin beds highly enriched in diatomaceous silica occur only sporadically in Holocene sediments of the western halistatic region (northwestern slope) (Shimkus et al., 1973). The DSDP site sediments contain from 1.02 to $18.94 \%$ $\mathrm{SiO}_{2 \text { amorph }}{ }^{1}$ or $1.58-22.64 \%$ on a carbonate-free basis.

A selective $\mathrm{SiO}_{2}$ amorph study of Hole 379A sediments shows the contents to range insignificantly from 1 to 5 percent (Table 18), indicating the insignificance there of diatomaceous phytoplankton as sedimentary components during the Quaternary.

In Site 380 Quaternary sediments, silica ranges mainly between 1.5 and $3.0 \%$ (Figure 9). Two peaks of slightly increased $(6.05 \%)$ and high $(13.15 \%)$

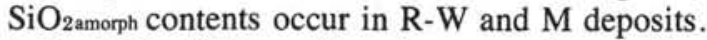

In Hole $380 \mathrm{~A}$ sediments, amorphous silica usually ranges from 1.5 to $4.0 \%$, and is markedly increased (Figure 11) in sediments of Eopleistocene (Units B-2 and B-3) and Pliocene (Units B-4-b and B-4-c) age.

$\mathrm{SiO}_{2}$ amorph at Site 381 makes up to $13.87 \%$, or $22.64 \%$ on a carbonate-free basis (Table 13); these are the maximum values of amorphous silica values for Black Sea sediments. In Units 3-4, small amounts of

TABLE 18

Percentage $\mathrm{SiO}_{2}$ Amorph

in Bottom Sediments, Hole 379A

Sample

(Interval in $\mathrm{cm}$ ) $\quad \mathrm{SiO}_{2}$ amorph

\begin{tabular}{ll}
\hline $1-4,115-125$ & 1.24 \\
$6-2,32-38$ & 1.40 \\
$7-2,20-30$ & 1.33 \\
$8-4,4-16$ & 1.26 \\
$9-5,50-58$ & 2.50 \\
$10-6,13-20$ & 1.58 \\
$11-5,53-55$ & 1.11 \\
$12-3,63-72$ & 1.11 \\
$16-3,17-27$ & 1.03 \\
$19-4,57-59$ & 2.26 \\
$21-6,0-10$ & 1.75 \\
$24-2,0-18$ & 1.23 \\
$24-6,62-74$ & 5.78 \\
$26-4,0-12$ & 1.31 \\
$31-3,106-108$ & 1.39 \\
$35-5,92-101$ & 1.66 \\
$37-3,33-35$ & 1.00 \\
$38-2,11-13$ & 1.60 \\
$40-2,14-24$ & 1.64 \\
$42-1,25-37$ & 1.48 \\
$45-2,108-110$ & 1.28 \\
$48-4,132-140$ & 1.15 \\
$50-5,12-24$ & 1.61 \\
$59-3,90-100$ & 1.52 \\
$68-5,73-75$ & 1.11 \\
\hline
\end{tabular}

${ }^{\prime} \mathrm{SiO}_{2}$ amorph $\times 1.6$.
$\mathrm{SiO}_{2}$ amorph are seen, although the content increases downward. Four beds enriched in silica occur in Units 5,6 , and 7 ; these are characterized, as a rule, by minimal $\mathrm{CaCO}_{3}$ contents and abundant organic matter. The $\mathrm{SiO}_{2}$ amorph distribution pattern here is similar to that at Hole 380A.

\section{Organic Carbon}

The Corg content of Recent sediments ranges between less than $1 \%$ to $5 \%$ (Shimkus and Trimonis, 1974) with maximum values observed in chalistatic regions. Corg contents below $1 \%$ and between 1 and $2 \%$ are found in modern sediments at Sites 379 and 380, respectively, and are derived from dinoflagellates and, to a lesser extent, coccolithosphoroids.

From Table 7 and Hirst's data (1974, Cores 1432 and 1462 ) it is evident that Holocene sediments in the vicinity of Site 379 contain a number of beds where $C_{\text {org }}$ ranges from 5 to $12.30 \%$; these are all concentrated in Old Black Sea (middle Holocene) horizons. Corg hardly reaches $1 \%$ in lower Holocene (late Neoeuxinic) sediments while in upper Würm (middle Neoeuxinic) it is often below $0.5 \%$.

Site 379: In Quaternary sediments of Site 379, Corg content ranges from $0.27 \%$ to $6.00 \%$; more often it is below $1 \%$, averaging $0.69 \%$. In terrigenous clay muds, in nannofossil oozes, and in muds enriched in diatomaceous silica, average contents are $0.54 \%, 0.55 \%$, and $1.60 \%$, respectively.

The occurrence of beds with increased and highCorg contents in the Interglacial units is the main phenomenon of $\mathrm{Corg}_{\mathrm{g}}$ distributions in the section. The interbeds are less enriched in organic matter in the upper Interglacial unit (4-5) (R-W) as compared with Unit M-R (1.44-2.76\% and $1.44-6.00 \%$ Corg, respectively).

The occurence of interbeds with Corg contents amounting to $0.87-2.04 \%$ is characteristic of the middle of Glacial unit 6 (R), representing warm, variable climatic periods within the Glacial epoch. Beds with increased Corg contents consist mainly of non-calcareous and low-calcareous clay muds; in places a diatomaceous silica content is higher.

Site 380: In Quaternary sediments, Corg contents range from 0.20 to $1.94 \%$; most often they are below $1 \%$, averaging $0.63 \%$. As at Site 379, the beds with high Corg contents (1.09 to $1.94 \%)$ occur in Intrglacial series, and are represented by non-calcareous clay muds in places enriched in diatomaceous silica.

Hole 380A: Corg contents in Eopleistocene sediments are the same as inlower Pleistocene ones of Site 380. In Neogene deposits they range from 0.11 to $3.18 \%$. Maximum amounts of organic matter are concentrated in Pliocene (Units B-4-a and B-4-b) where minimum values are in Miocene sediments (Unit B-5) (Table 13). The muds with Corg contents exceeding $1 \%$ are often enriched to a certain extent in diatomaceous silica.

Site 381: In lower Pleistocene sediments (Unit 3), small amounts of organic matter (more often $<0.5 \%$, rarely $0.5-0.8 \%$ of $\mathrm{C}_{\text {org }}$ are observed) ranging from 0.16 to $0.81 \%$. The range is sharply increased in Pliocene deposits, from 0.12 to $6.56 \%$ ( $1.12 \%$ average). The muds of the Pliocene units are most enriched in organic matter $(6.7 \%)$ compared with the corresponding Hole 
$380 \mathrm{~A}$ deposits, The highest Corg contents occur in beds of low-siliceous diatomaceous muds (Cores 36, 37).

Analyses of $\mathrm{C}_{\text {org }}$ and $\mathrm{SiO}_{2}$ amorph show a similar distribution of these components in both Quaternary and Neogene sediments: (1) Site 380 Interglacial units are to a certain extent enriched in these components; Hole 379A sediments are mainly enriched in organic matter, diatomaceous silica content being only relatively increased; (2) Corg and $\mathrm{SiO}_{2}$ amorph are most abundant in Pliocene sediments, the enrichment in both components being parallel but of differing magnitudes (Figure 24). This indicates a close genetic correlation between $\mathrm{C}_{\mathrm{org}}$ and $\mathrm{SiO}_{2}$ amorph.

\section{FEATURES OF ORGANIC MATTER AND DIATOMACEOUS SILICA- ENRICHED SEDIMENT FORMATION}

At the present stage of Black Sea development, enrichment of deep-water sediments in organic matter occurs in chalistatic regions, i.e., under the centers of surface water cyclonic gyres. Relatively high phytoplankton productivity (mainly dinoflagellates and coccolithophorids) and the low terrigenous influx result in relative enrichment in biogenic components (organic matter and carbonates), which explains the high $\mathrm{C}_{\text {org }}$ content of nannofossil oozes.

At certain times in the Holocene, the sediments were much more enriched in organic matter than at present. This is especially true of discrete single beds of Old Black Sea sediment. Maximum values (about 22\%) are observed in western chalistatic region (Shimkus et al., 1973).

Old Black Sea sapropel-like and sapropelic muds, like modern ones, formed in chalistatic regions, though their sedimentation was not accompanied by calcareous nannoplankton accumulation. These Old Black Sea muds are rather poor in carbonates as a result of a low coccolithoporid contribution in the phytoplankton.

Palynologic studies of a number of cores (Shimkus et al., 1975a, b) indicated that Old Black Sea sedimentation proceeded under a warm humid climate (more humid than at present) when maximum nutrient salt volumes were delivered from the drainage area. This resulted in rapid dinoflagellate blooms and in intensive deposition of planktogenic organic matter. It should be noted that Strakhov (1971) considers upwelling of Neoeuxinic deep waters, enriched in nutrient salts, due to displacement in heavy Mediterranean waters introduced through the Bosporus during the Holocene, as the chief reason for the great enrichment in organic matter of the Old Black Sea sediments. These conditions resulted in high surface productivity. Organic material derived low on the trophic web was introduced to the bottom deposits with little dissolution having occurred in the water column, a result of chelation of organics into crustacean faeces. Bottom anoxic conditions prevented in situ oxidation.

These organic muds were formed in areas of changing size and location in response to changes in the basin's hydrodynamic regime, hence an interbedding of sapropelous, clay-sapropelic, and sapropelic muds with clay muds is observed in the sections in different deepwater parts of the basin. As a result, periodic occurrences of muds rich in organic matter in peripheral areas of the basin zone as well as in central regions beyond the limits of the modern chalistatic region occurred. The same process is relevant to those Interglacial muds with increased Corg contents. Substantial organic matter has been destroyed during diagenetic processes, explaining the lower Corg

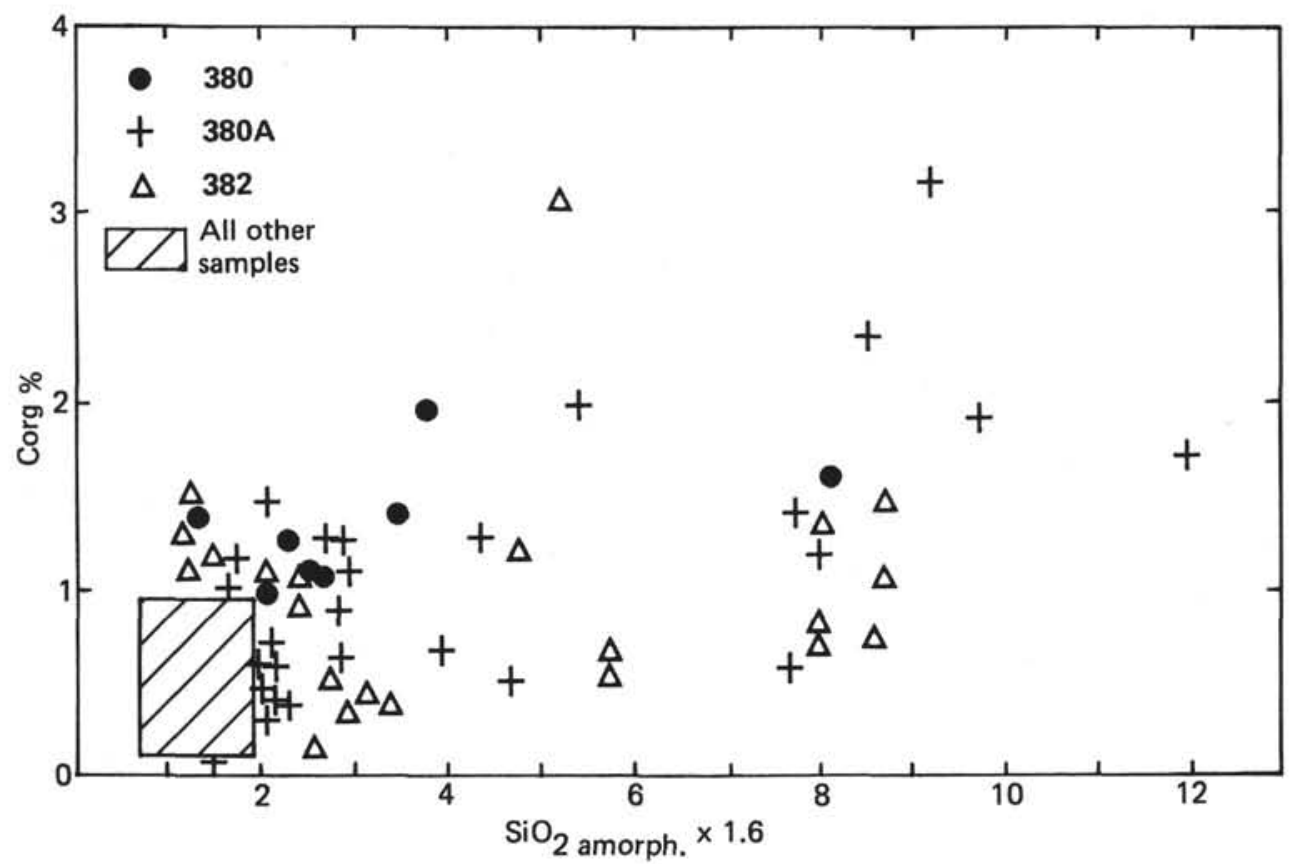

Figure 24. $\mathrm{C}_{\text {org }}$ distribution in site sediments as a function of $\mathrm{SiO}_{2 \text { amorph }}$ contents. 
contents in Quaternary and Pliocene sediments, as is evidenced by high pyrite and diagenetic carbonate contents. A contributing process is large-scale dilution of these sediments by terrigenous material.

Non-calcareous or low-calcareous beds of Quaternary muds enriched in organic matter are characteristic of Hole 379A and are similar to Old Black Sea sediments. This suggests possible similar processes of formation. Late Pliocene and early Pleistocene sapropelic muds were dredged from bedrock deposits on the slope opposite Sinop. $\mathrm{CaCO}_{3}$ contents of these samples ranges from 8.2 to $28.0 \%$ with $\mathrm{C}_{\text {org }}$ content averaging $11 \%$. The high calcareous consolidated muds consist mainly of nannoplankton.

Beds with increased $\mathrm{C}_{\text {org }}$ contents comprise noncalcareous and low-calcareous muds in Sites 380 and 381 Pleistocene-Eopleistocene sediments and high calcareous muds in units within Pliocene sediments (Site 381, Unit 7). As stated earlier, some interbeds are also enriched in diatomaceous silica. Thus, at these sites sediment enrichment occurs either in $\mathrm{C}_{\text {org }}$ alone or together with diatomaceous silica.

Enrichment of muds in both $\mathrm{C}_{\text {org }}$ and $\mathrm{SiO}_{2}$ amorph is observed in Holocene sediments of the western chalistatic region, where siliceous, organic calcareous, and clay materials alternate (Shimkus et al., 1973). Dinoflagellates contribute significant organic matter to these beds, as is also the case for the sapropelic diatomaceous muds at the drill sites.

Beds considerably enriched in amorphous silica but poor in organic matter occur in Pliocene-Eopleistocene sediments and in lower Interglacial series. In the Holocene sediment, they co-exist with beds enriched both in $\mathrm{C}_{\text {org }}$ and $\mathrm{SiO}_{2}$ amorph.

Both in the Holocene and Pleistocene cases, the muds with increased diatomaceous contents are typical only of the western part of the basin (such muds were discovered in only one interbed at Site 379). Analyses of Holocene diatomaceous muds (Shimkus, et al., 1973) showed that this fact is not fortuitous, rather it is related to strong nutrient salt delivery by the large rivers of the northwestern drainage system. According to Lisitzin (1964), a diatom abundance considerably higher than that of dinoflagellates indicates conditions of maximum nutrient salt delivery.

Diatomaceous muds, like sapropelic ones are formed in halistatic regions with low dilution of silica by terrigenous material. An important condition for diatomaceous mud formation is the absence of biogenic carbonates. For example, a cool environment is unfavorable for coccolithophorid development.

Silica, like organic matter, is more intensively concentrated in sediments when the major part of it is transported from the surface productive layer to the bottom as faeces, as is characteristic where diatoms are heavily grazed by copepods.

Thus, diatom muds formed during periods of intensive nutrient delivery from the drainage system and coincident water cooling, when the western chalistatic region extended to Holes $380 / 380 \mathrm{~A}$ and 381 .

\section{Iron Group (Fe, $\mathrm{Mn}, \mathrm{Ni}, \mathrm{Co}) \mathrm{Cu}, \mathrm{Mo}, \mathrm{W}, \mathrm{Se}$}

The group consists of geochemically mobile elements. In the Black Sea rivers, they mainly move as suspended matter, though significant amounts of the elements are transported in the solution as hydroxides (or their components) and organometallic components (Glagovela, 1959; Lubchenko and Belova, 1973; Strakhov et al., 1971; Volkov, 1975). They are associated with the most finely dispersed suspended matter and the pelagic maximums. It should be noted that the fields of maximum contents of the elements do not completely coincide since the main source areas of the various elements are situated in different parts of the drainage area.

The localization of maximum content values (see figures by Strakhov et al., 1971) suggests that the eastern Pontic area is an important source area of $\mathrm{Fe}$ and $\mathrm{Ni}$, whereas the northwestern drainage area is a source of $\mathrm{W}$ and Se. High concentrations of other elements are caused by material supplied from both these areas and the Caucasus.

The group takes an active part in diagenetic exchange, forming either authigenic minerals or occurring as isomorphic admixtures in them.

\section{Iron}

Ranges of iron in Miocene-Quaternary sediments are extremely wide, from 0.65 to $35.50 \%$ (Table 19). The maximum $\mathrm{Fe}$ content is thus 47 times higher than the minimum. This wide range of $\mathrm{Fe}$ content is found in neither Recent nor late Quaternary sediments (Glagoleva, 1961); the average Fe content at the drill sites are much higher than those for the Recent sediments (Table 9). A noticeable ferruginous enrichment of the Miocene-Pleistocene sediments is evident compared to Holocene values.

$\mathrm{Fe}$ values for Hole 379A are low, comparable to the element concentration in the local surface sediments, suggesting both a comparative stability of source areas and sedimentation conditions for the main part of Pleistocene and Holocene and a weak diagenesis resulting in $\mathrm{Fe}$ assimilation in some interbeds of other sites.

The Fe content range in Hole 379A units (on a carbonate-free basis), from 5.06 to $5.94 \%$ (Table 4 ), is narrow. The lowest values are found in Units 4-5 (R$\mathrm{W})$, in terrigenous muds containing high diatom Corg and feldspar contents and low amounts of clay minerals. The highest average $\mathrm{Fe}$ contents were found in Unit 7 (H-R), 5.69\%. Because this unit in mineral composition is almost the same as the other units, the higher $\mathrm{Fe}$ (compared to the average values) may be due to finely dispersed $\mathrm{Fe}$ oxides; pyrite in some samples (e.g., 29-5, 4-6 cm) is as high as $7.8 \%$.

At Site $380, \mathrm{Fe}$ content ranges and average values (Table 11) are much higher than at Hole 379A, probably due to more variable sedimentation conditions in the region of Site 380 and to more intensive diagenesis. With beds enriched in diagenetic 
TABLE 19

Average Contents of the Ferric Group Elements (Fe, Mn, Ni, Cu, Co)

\begin{tabular}{|c|c|c|c|c|c|c|c|c|c|c|c|}
\hline \multirow{3}{*}{ Hole } & & \multicolumn{4}{|c|}{ Contents (\%) } & \multicolumn{6}{|c|}{ Content $\left(10^{-4} \%\right)$} \\
\hline & & \multicolumn{2}{|l|}{$\mathrm{Fe}$} & \multicolumn{2}{|c|}{ Mn } & \multicolumn{2}{|c|}{$\mathrm{Ni}$} & \multicolumn{2}{|c|}{ Co } & \multicolumn{2}{|c|}{$\mathrm{Cu}$} \\
\hline & & 1 & 2 & 1 & 2 & 1 & 2 & 1 & 2 & 1 & 2 \\
\hline \multirow{2}{*}{$379 \mathrm{~A}$} & a & $2.26-7.14$ & 4.35 & $0.03-0.34$ & 0.09 & 23-206 & 139 & $10-53$ & 34 & $24-128$ & 50 \\
\hline & $\mathrm{b}$ & $2.94-7.33$ & - & $0.03-0.47$ & - & $28-254$ & - & - & - & $29-147$ & - \\
\hline \multirow{2}{*}{380} & a & $1.88-8.24$ & 4.39 & $0.03-0.98$ & 0.10 & $30-98$ & 76 & $3-33$ & 19 & $13-61$ & 39 \\
\hline & b & $2.14-9.48$ & 5.12 & $0.04-1.13$ & 0.12 & 53-119 & 89 & $5-37$ & 22 & $20-77$ & 45 \\
\hline \multirow{2}{*}{$380 \mathrm{~A}$} & a & $1.48-35.50$ & 4.28 & $0.02-3.65$ & 0.15 & $38-88$ & 58 & $7-40$ & 14 & $33-55$ & 41 \\
\hline & b & $1.55-39.67$ & 6.03 & $0.21-4.08$ & 0.21 & $53-155$ & 83 & $7-41$ & 20 & $38-150$ & 58 \\
\hline \multirow{2}{*}{381} & a & $0.65-35.00$ & 4.64 & $0.01-5.43$ & 0.17 & $18-180$ & 62 & $3-30$ & 20 & - & - \\
\hline & b & $0.72-45.01$ & 6.14 & $0.01-6.98$ & 0.22 & $54-189$ & 82 & $5-34$ & 26 & - & - \\
\hline \multirow{2}{*}{ All holes } & a & $0.65-35.50$ & - & $0.01-5.43$ & - & $18-200$ & - & $3-53$ & - & $13-128$ & - \\
\hline & b & $0.72-45.01$ & - & $0.01-6.98$ & - & 53-189 & - & $5-41$ & - & $20-150$ & - \\
\hline
\end{tabular}

Note: $\mathrm{a}=$ natural sediment $\mathrm{b}=$ on $\mathrm{a}$ carbonate free basis; $1=$ range; $2=$ average .

minerals (carbonate, sulfides) being excluded from the calculations, the average $\mathrm{Fe}$ contents are similar to those of the surface layer of sediment.

Iron content ranges in Hole $380 \mathrm{~A}$ are even wider than in Hole 380 (Table 19) but average Fe contents in some sediment units are rather variable (Table 14). The high contents occur in Unit B-4-a. In general, Fe contents markedly decrease from top to bottom, where $\mathrm{CaCO}_{3}$ increases, i.e., $\mathrm{Fe}$ has an inverse correlation with $\mathrm{CaCO}_{3}$ (Figure 11). Fe contents on a carbonatefree basis are markedly uniform.

Beds of unit B-4-a contain 16.3 to $35.5 \% \mathrm{Fe}$ and $3.65 \% \mathrm{Mn}$. Examination of smear slides and thin sections shows large amounts of ferruginous chemogenic-diagenetic carbonates such as ankerite and siderite.

At Site $381 \mathrm{Fe}$ content ranges also are extremely wide and the average value for this site is the maximum for the Black Sea (Table 19). Element contents differ in the different units (Table 16). They are minimum for Unit 8 and maximum for Unit 5. In the latter, two interbeds with 19.55 and $35.00 \%$ of $\mathrm{Fe}$ and 1.84 and $5.43 \%$ of $\mathrm{Mn}$ contents, respectively, were found. Beds with high $\mathrm{Fe}$ $(>6 \%)$ and $\mathrm{Mn}$ contents occur in other units as well. High $\mathrm{Fe}$ contents are usually associated with ferruginous carbonates, among which are siderite and ankeritic dolomite (Tables 20, 21). Mangano-siderite and rhodochrosite(?) may also occur. Fe contents in most samples are directly proportional to siderite content (Table 21, Figure 25), suggesting that the diagenetic carbonates are the carriers of $\mathrm{Fe}$.

\section{Manganese}

The overall range of $\mathrm{Mn}$ is 0.01 to $5.43 \%$. The highest average contents are found in Site 381 , whereas the lowest occur in Hole 379A (Table 19).

Mn content in Hole 379 does not have a wide range and its average content in some units is similar to that of the upper layer of Black Sea bottom sediments (Table 9, Figure 7; see Glagoleva, 1961, fig. 6). Mn does not show a direct correlation with $\mathrm{Fe}$ when its content exceeds $0.10 \%$ and when its significant (or main) part is represented by mobile forms. In such cases $\mathrm{Mn}$ "separates" from $\mathrm{Fe}$ and forms other minerals (e.g.,
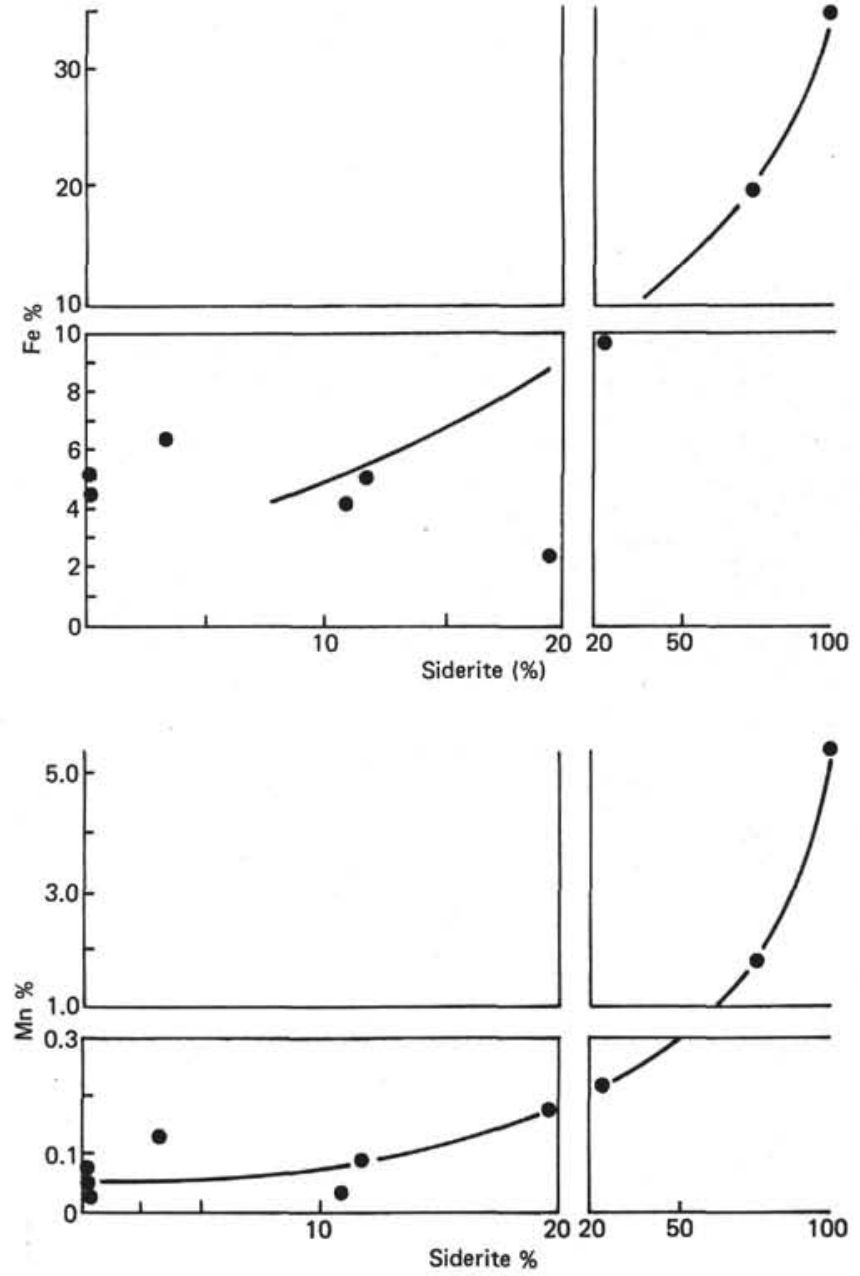

Figure 25. Fe and Mn distribution in Site 381 sediments as a function of siderite contents.

Mn hydroxides may be dispersed in the sediments). Significant amounts of $\mathrm{Mn}$ sometimes occur independent of terrigenous matter, evidenced by the rather clear but inverse correlation of $\mathrm{Mn}$ with $\mathrm{K}$ (an indicator of terrigenous matter). In the lower part of the site, $\mathrm{Mn}$ shows a direct correlation with $\mathrm{CaCO}_{3}$, 
either a result of dispersed $\mathrm{Mn}$ in the calcareous beds or by $\mathrm{Mn}$ substitution in the lattice during chemogenicdiagenetic carbonate formation.

Average Mn contents at Site 380 are the same as those at Site 379 , but their ranges are much wider (Table 19). The highest Mn contents were found in terrigenous low-ferruginous sediments. As neither siderite, dolomite (all carbonates are entirely represented by calcite) nor pyrite were found in the one sample studied $(27-3,1-13 \mathrm{~cm})$, it may be that $\mathrm{Fe}$ and Mn occur as hydroxides.

In contrast to Hole $379 \mathrm{~A}, \mathrm{Mn}$ at Site 380 is distributed as is $\mathrm{Fe}$ (Figure 9), e.g., a direct correlation exists between these elements. This may mean that $\mathrm{Fe}$ and $\mathrm{Mn}$ are contained in the same, probably terrigenous, minerals.

Hole $380 \mathrm{~A}$ sediments contain from 0.02 to $3.65 \% \mathrm{Mn}$ (Table 19), but if the maximum value is excluded, the upper limit of $\mathrm{Mn}$ is $0.35 \%$ (see Sample 69-4, 53-58 cm). $\mathrm{Mn}$ contents most often range from 0.05 to $0.15 \%$, which is somewhat higher than the range at Hole 379A. Thus, there is an enrichment of Hole $380 \mathrm{~A}$ sediments in both $\mathrm{Fe}$ and $\mathrm{Mn}$. Maximum accumulation of $\mathrm{Mn}$ is observed in Unit B-4-a which contains chemogenicdiagenetic carbonates; in sediments containing 20-100\% of ankerite-siderite, $0.22-5.43 \%$ of $\mathrm{Mn}$ is found.

At Hole $379 \mathrm{~A}$ correlation between $\mathrm{Mn}$ and $\mathrm{Fe}$ is insignificant (Tables 22, 23) or does not exist at all, which may be evidence of these elements existing in different minerals. Only in a single sample with maximum $\mathrm{Fe}$ content was maximum $\mathrm{Mn}$ also found; here both elements are probably associated with complex carbonates of the ankerite-siderite-type (manganosiderite?), which can constitute up to $80-100 \%$ of the crystalline phase.

The widest ranges of $\mathrm{Mn}$ content as well as highest average values, were observed at Site 381 (Table 19). Particularly high contents of the element (as well as that of $\mathrm{Fe}$ ) were found in Units 4 and 5 (Table 16); either siderite beds or sediments rich in siderite occur. At this site, a direct relationship between $\mathrm{Mn}$ and siderite content is observed. Mn maximum concentration (5.4\%) was found in a sample consisting of $100 \%$ siderite (Table 2I). This suggests that $\mathrm{Mn}$, as well as Fe, is contained in the crystalline lattice of diagenetic carbonates. Those samples without carbonates $(0.00 \%$ of $\left.\mathrm{CaCO}_{3}\right)$ are characterized by minimum amounts of $\mathrm{Mn}(0.01$ to $0.06 \%)$ with a rather significant $\mathrm{Fe}$ content (3.56 to $5.55 \%$ ), suggesting a different geochemical behavior of the elements during diagenesis.

\section{Nickel}

$\mathrm{Ni}$ content in the sediments ranges from 18 to 200 ppm, i.e., lower than Fe and Mn but still rather high; maximum content is 21 times higher than the minimum (Table 10).

Both the maximum and highest average contents of $\mathrm{Ni}$ were found in Hole 379A where they approach maximum values in Recent sediments. High $\mathrm{Ni}$ contents were found over the whole section at this site. Quaternary sediments (Strakhov et al., 1971; fig. 8) as opposed to Recent and late Quaternary muds in the region of Hole 379A (Hirst, 1974) are characterized by high Ni contents; the late Quaternary muds sampled eastwards of Hole 379A (north of Inebolu, Station 4752-2) have markedly lower Ni contents (Table 7). Sedimentary material, enriched in $\mathrm{Ni}$ as a result of erosion of ultrabasic rocks in the Kizyl-Irmak drainage area, was transported to the vicinity of Site 379 , a probability corroborated by the similarity of $\mathrm{Ni}$ content and $\mathrm{K}_{\mathrm{b}}$ values. Because $\mathrm{Ni}$ in the Site 379 region accumulated in larger quantities in the Pleistocene than at present, the Pleistocene drainage pattern (sediment load) of the Kizyl-Irmak has probably changed.

The close correlation between $\mathrm{Ni}$ and $\mathrm{Ti}$ observed in most of the Hole 379A section (Tables 22, 23) suggests a genetic relation of $\mathrm{Ni}$ to the weathered products of basic volcanogenic material (montmorillonite etc., Figure 26) during almost all of the Pleistocene. This correlation is broken only for Unit $8(\mathrm{M})$, probably due to intensive supply of sedimentary material by not only the Pontic rivers but by northwestern and Caucasian rivers as well.

Analysis of the correlation series shows that in the Neogene-Quaternary sediment, $\mathrm{Ni}$ deviates from $\mathrm{Fe}$ and $\mathrm{Mn}$. This is caused by fractionation of $\mathrm{Ni}$ during sediment diagenesis; it is intensively redistributed and concentrated in the sulfides. Though the correlation between $\mathrm{Ni}$ and pyrite is far from being close (Figure 27 ), it is marked. In sedimentation processes, $\mathrm{Ni}$ is closely associated with montmorillonite and other erosion products of basic and ultrabasic rocks. This association is not fully eradicated by diagenesis.

Rather low Ni contents (Tables 11, 14) are found in the Neogene-Quaternary sediments from Holes 380 and $380 \mathrm{~A}$ (as well as in the Recent sediments in the region). This is a result of the fact that sediment carried by the Danube is impoverished in $\mathrm{Ni}$. Low $\mathrm{Ni}$ content also characterizes the upper part of Site 381, but in the Pliocene and Miocene sediments (Units 6, 9, 10,11) Ni contents are noticeably increased, approaching the average values for Hole 379A. During the formation of these Miocene-Pliocene deposits, large amounts of material deposits were probably contributed by sources additional to the Danube.

\section{Cobalt}

Cobalt content varies from 3 to $53 \mathrm{ppm}$ and the average values are very close to Ko.

Sediments from Hole 379A (Table 24) are enriched in cobalt as well as in Ni. Maximum cobalt content occurs in Unit $8(\mathrm{M})$, terrigenous low-calcareous muds with little organic matter. Unit 8 sediments contain chlorite about $15-25 \%$ higher than Units 3, 4, 5. Because chlorite is genetically associated with the river loads of the middle Pontic and the Great Caucasus Rivers (including the Kuban) (see discussion by Muller and Stoffers, 1974), we conclude that sediments carried by the Caucasian rivers as well as from Pontic drainage area accumulated here. The finely dispersed part of this sediment load seems to be enriched in Co.

In Holes 380 and 380A Co contents are much lower than at Sites 379 and 381 . The upper part of Site 381 is also characterized by low Co but in the middle and 
TABLE 20

Chemical Composition of Low Ferruginous $(6-10 \% \mathrm{Fe})$ and Ferruginous $(>10 \% \mathrm{Fe})$ Sediments of the Black Sea, Containing Considerable Amounts of Chemogenic-Diagenetic Carbonates or Other Authigenic Minerals

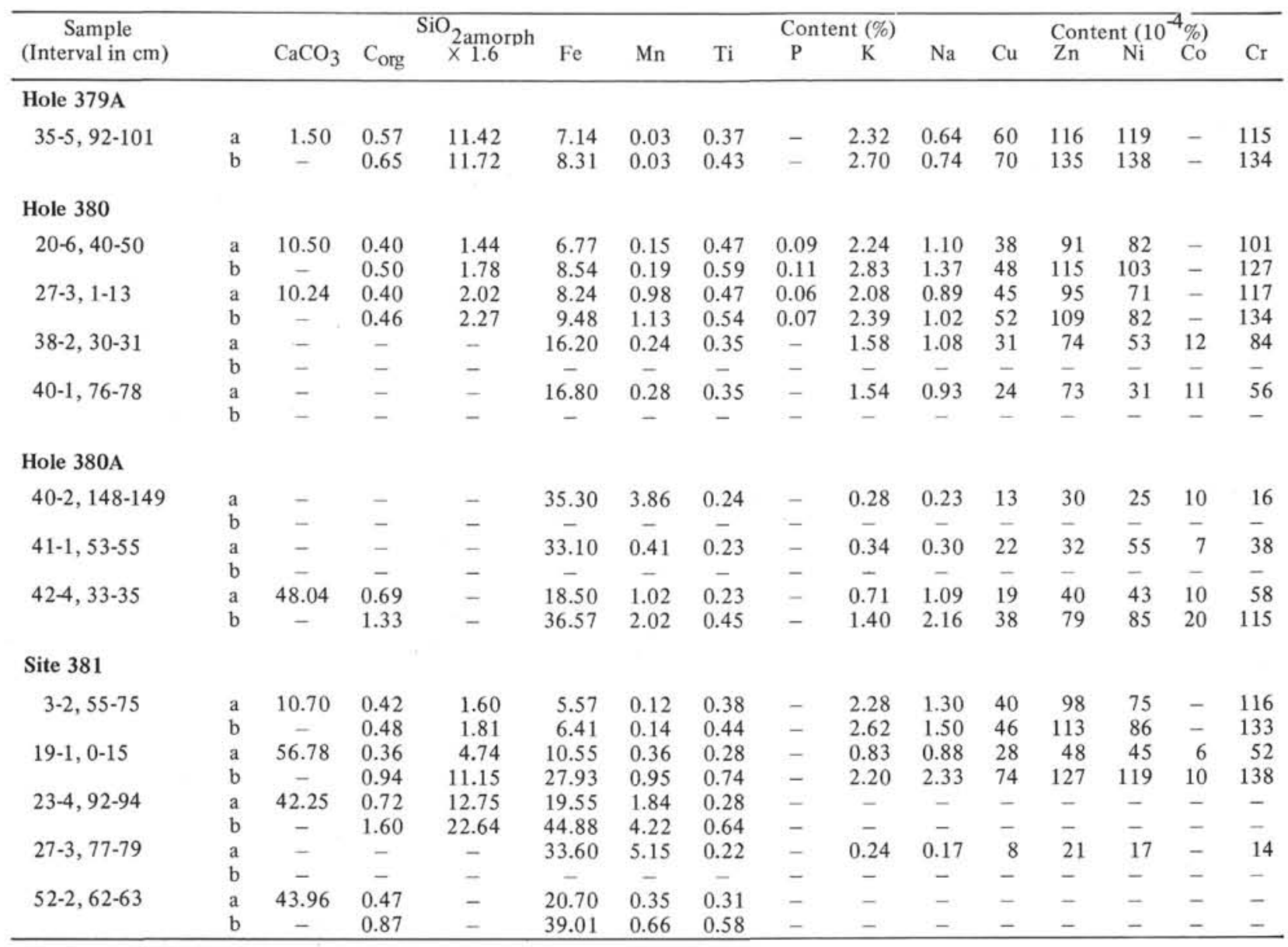

Note: $a=$ natural sedimen $; \quad b=$ on a clastic matter basis.

lower part, Co contents are markedly increased (Tables 15, 16); the same reason applies as for $\mathrm{Ni}$.

\section{Copper}

Copper has a wide range in Recent sediments. Low values $(<100 \mathrm{ppm})$ occur in peripheral regions of the Black Sea and maximum values occur in chalistatic regions (Strakhov et al., 1971). Maximum content distributions indicate that the Dnieper in the Western Sea, and the Caucasian and Pontic rivers in the eastern part are the main copper sources.

$\mathrm{Cu}$ contents range from 24 to $128 \mathrm{ppm}$ in MioceneQuaternary sediments and are close to $\mathrm{K}_{\mathrm{o}}$ or slightly lower. Values are mainly low, never reaching the levels observed in Recent sediments. $\mathrm{Cu}$ distribution is irregular down section with marked variations in the Interglacial series.

Relatively high $\mathrm{Cu}$ contents occur in those beds enriched in organic matter (except for low-calcareous diatomaceous muds, Table 24, Figure 28). This pattern is also characteristic of Holocene sediments in the site areas, as shown by Volkov and Fomina (1974), Hirst (1974), and by our data (Table 7). Thus, Cu content variations are to a considerable extent dependent on both $\mathrm{C}_{\text {org }}$ and sulfide distribution (Figure 27).

\section{Molybdenum}

Mo (as well as W and Se) was studied only in Site 380 sediments (Table 10). Its content ranges from 0.8 to $12.4 \mathrm{ppm}$, the average value being 2.6. Throughout the site the Mo content is almost the same as that of the upper Black Sea sediment layer (Table 9). Single units contain from 1.3 to $4.8 \mathrm{ppm}$ of Mo, or 1.5 to $5.1 \mathrm{ppm}$ on a carbonate-free basis (average value $3.0 \mathrm{ppm}$ ). This value is noticeably higher than Mo clarke for clays and shales $\left(\mathrm{K}_{\mathrm{o}}\right)$. A low Mo content was found in Sample 30$1,94-110 \mathrm{~cm}$, ferrugenous low-calcareous muds, and high Mo was found in diatomaceous muds enriched in organic matter (Sample 35-3, 19-42 cm) and containing $3 \%$ pyrite.

In general, Mo is found to have a close correlation with $\mathrm{C}_{\text {org }}$ and partly with sulfides in the Recent and Holocene sediments (Pilipchuk, 1971; Pilipshuk and Volkov, 1974; Volkov and Fomina, 1974) and in the entire Pleistocene sediment section (Table 10). Indeed, Mo is found to form complexes with organic matter while still in the water column. An original sorptive link apparently becomes a chemical bond.

During diagenesis the organic Mo forms may be transformed into inorganic (sulfide) forms according to the scheme: 
TABLE 20 - Continued

\begin{tabular}{|c|c|c|c|c|c|c|c|c|c|c|c|c|c|c|c|c|c|c|c|}
\hline V & $\mathrm{Li}$ & $\mathrm{Rb}$ & Cs & $\mathrm{Pb}$ & $\mathrm{Sn}$ & $\mathrm{Cd}$ & $\mathrm{Se}$ & Mo & W & $\mathrm{F}$ & B & $\mathrm{La}$ & Th & $\mathrm{Hf}$ & $\mathrm{Sc}$ & Ew & $\mathrm{Sb}$ & $\mathrm{Ce}$ & $\mathrm{Ta}$ \\
\hline - & - & - & - & - & - & $<6$ & - & - & - & - & - & - & - & - & - & - & - & - & - \\
\hline - & - & - & - & - & - & 7 & - & - & - & - & - & - & - & - & - & - & - & - & - \\
\hline - & 45 & 80 & 6.0 & 16 & 2.2 & $<5$ & 0.4 & 1.2 & 2.5 & - & - & - & - & - & - & - & - & - & - \\
\hline - & 57 & 101 & 7.6 & 20 & 2.8 & $<6$ & 0.5 & 1.5 & 3.2 & - & - & - & - & - & - & - & - & - & - \\
\hline- & 45 & 100 & 6.0 & 20 & 2.2 & $<5$ & 0.3 & 1.2 & 20.0 & - & - & - & - & - & - & - & - & - & - \\
\hline- & 52 & 115 & 6.9 & 23 & 2.5 & $<6$ & 0.3 & 1.4 & 23.0 & - & - & - & - & - & - & - & - & - & - \\
\hline 50 & - & - & - & - & - & $<5$ & - & 23.0 & - & - & - & 29 & 6.0 & 3.5 & 15 & 1.3 & 0.5 & 70 & - \\
\hline- & - & - & - & - & - & - & - & - & - & - & - & - & - & - & - & - & - & - & - \\
\hline 50 & - & - & - & - & - & $<4$ & - & 6.3 & - & - & - & 24 & 7.0 & - & 15 & 0.9 & 1.7 & 67 & 0.2 \\
\hline- & - & - & - & - & - & - & - & - & - & - & - & - & - & - & - & - & - & - & - \\
\hline 50 & 40 & 70 & 5.0 & 18 & 2.0 & $<4$ & - & 24.0 & - & - & - & - & - & - & - & - & - & - & - \\
\hline- & - & - & - & - & - & - & - & - & - & - & - & - & - & - & - & - & - & - & - \\
\hline 50 & - & - & - & - & - & 10 & - & 5.0 & - & _- & - & 3 & - & - & - & - & - & - & - \\
\hline- & - & - & - & - & - & - & - & - & - & - & - & - & - & - & - & - & - & - & - \\
\hline 50 & - & - & - & - & - & $<4$ & - & 5.0 & - & - & - & - & 1.5 & 1.4 & 9 & 2.9 & - & - & 0.9 \\
\hline 99 & - & - & - & - & - & 8 & - & 9.9 & - & - & - & - & 3.0 & 2.8 & 18 & 5.7 & - & - & 1.8 \\
\hline 130 & 16 & 70 & 4.8 & 16 & 1.6 & $<4$ & - & 5.0 & - & - & - & - & - & - & - & - & - & - & - \\
\hline 150 & 18 & 81 & 5.5 & 18 & 1.8 & $<5$ & - & 5.8 & - & - & - & - & - & - & - & - & - & - & - \\
\hline 50 & 30 & 60 & 6.4 & 3 & - & $<6$ & - & 5.0 & - & 500 & - & 10 & 4.0 & 1.1 & 10 & 0.3 & 0.5 & - & - \\
\hline 132 & 79 & 159 & 16.9 & 8 & - & 16 & - & 13.2 & - & 1324 & - & 26 & 11.0 & 2.9 & 26 & 0.8 & 1.32 & - & - \\
\hline- & - & - & - & - & - & - & - & - & - & - & - & - & - & - & - & - & - & - & - \\
\hline - & - & - & - & - & - & - & - & - & - & - & - & - & - & - & - & - & - & - & - \\
\hline 50 & - & - & - & - & - & $<4$ & - & 8.2 & - & - & - & - & - & - & - & - & - & - & - \\
\hline - & - & - & - & - & - & - & - & - & - & - & - & - & - & - & - & - & - & - & - \\
\hline - & - & - & - & - & - & - & - & - & - & - & - & - & - & - & - & - & - & - & - \\
\hline- & - & - & - & - & - & - & - & - & - & - & - & - & - & - & - & - & - & - & - \\
\hline
\end{tabular}

$$
\mathrm{Mo} \text { (hum) } \mathrm{Na}+\mathrm{nS}^{2} \rightarrow \underset{\left[\mathrm{MoO}_{4-\mathrm{n}}-\mathrm{Sn}\right]^{2-}}{\left[\mathrm{MoSO}_{4}\right]^{-2}}
$$

which explains the Mo and sulfide association.

Besides this process, Mo is extracted from marine water by the dispersed ferruginous sulfides (Pilipchuk, 1971) as $\left(\mathrm{MoS}_{4}\right)^{2-}$ and $\left(\mathrm{MoO}_{4-\mathrm{n}} \mathrm{S}_{\mathrm{n}}\right)^{2-}$, as well as $\mathrm{FeS} \cdot \mathrm{MoS}_{3} \cdot \mathrm{n} \cdot \mathrm{H}_{2} \mathrm{~S}$. A similar process may take place in the bottom sediments during reaction of hydrotroillite and thiomolybdenic ions. During sediment diagenesis, dispersed ferruginous hydrotroillite-monosulfide is transformed into disulfide-pyrite $\left(\mathrm{FeS} \cdot \mathrm{MoS}_{3} \cdot \mathrm{nH}_{2} \mathrm{~S} \rightarrow \mathrm{FeS}_{2} \cdot \mathrm{MoS}_{3}\right)$ and probably into other forms.

\section{Tungsten}

Site 380 tungsten ranges from 10 to $40 \mathrm{ppm}$ (average value being $18 \mathrm{ppm}$ ) which is much higher than its $\mathrm{K}_{\mathrm{o}}$ (Tables 9, 10) and is similar to its value for the upper Black Sea sedimentary layers (Pilipchuk and Volkov, 1966). Minimum contents of the element (10 to $13 \mathrm{ppm}$ ) are found in terrigenous low-calcareous muds $(10-30 \%$ of $\left.\mathrm{CaCO}_{3}\right)$; maximum (30-40 ppm) content occurs in non-calcareous muds $\left(<10 \%\right.$ of $\left.\mathrm{CaCO}_{3}\right)$.
Calcium carbonate dilutes W concentrations, and on a carbonate-free basis the W content ranges from 11 to $42 \mathrm{ppm}$ with an average value of $23 \mathrm{ppm}$. Maximum average contents of $\mathrm{W}$ occur in Unit B-1-g muds, i.e., the Glacial sediments.

\section{Selenium}

In Site 380 sediments, Se ranges from 0.2 to $3.3(0.5 \%$ on the average) which are values similar to that of the upper Black Sea sedimentary layers (Sokolova and Pilipchuk, 1970) and about 10 times higher than the clarke for $\mathrm{Se}\left(\mathrm{K}_{\mathrm{o}}\right)$. Minimum amounts of $\mathrm{Se}(0.2 \%)$ were found in the terrigenous low-calcareous muds of Unit B-1-c (W), characterized by low contents of $\mathrm{C}_{\mathrm{org}}(0.20$ $0.56 \%)$. Higher selenium contents $(1.8$ and $1.4 \%)$ are characteristic of the terrigenous sediments with more $\mathrm{C}_{\mathrm{org}}(1.42-1.64 \%$ ) from Samples 35-3, 19-42 cm and 36$2,28-38 \mathrm{~cm}$. Pyrite occurs in both samples $(2.9$ and $3.0 \%$, respectively). The distribution of Se at this site is more like that of Mo than to $\mathrm{W}$ and the average Mo and $\mathrm{Se}$ contents in Neoeuxinic sediments and those of Site 380 are very similar. For Mo, these values practically coincide $\left(2 \times 10^{-4 \%}\right)$, as is true of $\mathrm{C}_{\mathrm{org}}$ $(0.7 \%)$, whereas the average Se contents at Site 380 and Neoeuxinic sediments differ from each other only by $3 \%$. The average values of $\mathrm{Mo}, \mathrm{Se}$, and $\mathrm{C}_{\text {org }}$ in Site 380 
TABLE 21

Mineral Composition of Sediments Enriched in $\mathrm{Fe}$ and $\mathrm{Mn}$

\begin{tabular}{|c|c|c|c|c|c|c|c|c|c|c|c|c|c|c|c|}
\hline $\begin{array}{c}\text { Sample } \\
\text { (Interval in } \mathrm{cm} \text { ) }\end{array}$ & 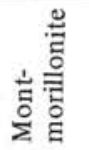 & 弚 & $\begin{array}{l}\stackrel{ }{\sharp} \\
\text { 롤 }\end{array}$ & 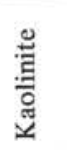 & $\frac{\stackrel{\Xi}{0}}{\text { J }}$ & 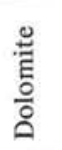 & 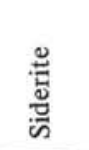 & 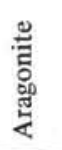 & 芶 & 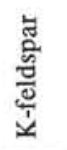 & $\begin{array}{l}\mathscr{\%} \\
\frac{\pi}{0} \\
\frac{0}{60} \\
\frac{\pi}{2}\end{array}$ & : & $\begin{array}{l}\text { E } \\
\text { 心 } \\
\text { 心 }\end{array}$ & $\mathrm{Fe}$ & $\mathrm{Mn}$ \\
\hline & & & \\
\hline $380-27-3,1-13$ & 7.4 & 45.2 & 11.9 & 2.4 & 9.0 & 0.0 & 0.0 & 0.0 & 14.5 & 4.0 & 5.6 & 0.0 & 0.0 & 8.24 & 0.98 \\
\hline $381-3-2,55-75$ & 11.1 & 29.4 & 13.3 & 4.9 & 12.1 & 0.0 & 3.3 & 0.0 & 17.5 & 0.0 & 8.2 & 0.0 & 0.0 & 6.28 & 0.13 \\
\hline $381-7-4,125-145$ & 27.4 & 25.6 & 11.4 & 3.4 & 13.6 & 2.1 & 0.0 & 0.0 & 11.4 & 0.0 & 5.1 & 0.0 & 0.0 & 6.30 & - \\
\hline $381-19-1,0-15$ & 9.5 & 5.3 & 3.1 & 0.0 & 53.5 & tr. & 23.8 & 0.0 & 4.8 & tr. & tr. & tr. & 0.0 & 9.66 & 0.22 \\
\hline $381-23-4,92-94$ & 12.2 & 10.4 & 0.0 & 0.0 & 0.0 & 0.0 & 74.2 & 0.0 & 3.2 & 0.0 & 0.0 & 0.0 & 0.0 & 19.55 & 1.84 \\
\hline $381-27-3,77-79$ & 0.0 & 0.0 & 0.0 & 0.0 & 0.0 & 0.0 & 100.0 & 0.0 & 0.0 & 0.0 & 0.0 & 0.0 & 0.0 & 35.00 & 5.43 \\
\hline $381-52-2,65-67$ & 7.3 & 36.7 & 23.1 & 3.1 & 0.0 & 0.0 & tr. & 0.0 & 14.6 & 5.7 & 9.5 & 0.0 & 0.0 & - & - \\
\hline
\end{tabular}

TABLE 22

Correlation Coefficients of Elements in Sediments of Würm (Unit 3) and Riss-Würm (Units 4, 5), Hole 379A

\begin{tabular}{|c|c|c|c|c|c|c|c|c|c|c|c|c|c|}
\hline & $\mathrm{CaCO}_{3}$ & $\mathrm{C}_{\text {org }}$ & $\mathrm{Fe}$ & $\mathrm{Mn}$ & $\mathrm{Ti}$ & $\mathrm{K}$ & $\mathrm{Na}$ & $\mathrm{Cu}$ & $\mathrm{Zn}$ & $\mathrm{Ni}$ & $\mathrm{Cr}$ & $\mathrm{x}$ & $\mathrm{E}_{\mathrm{x}}$ \\
\hline \multicolumn{14}{|c|}{ Unit 3 (Würm) } \\
\hline $\begin{array}{l}\mathrm{CaCO}_{3} \\
\mathrm{C}_{\text {org }} \\
\mathrm{Fe} \\
\mathrm{Mn} \\
\mathrm{Ti} \\
\mathrm{K} \\
\mathrm{Na} \\
\mathrm{Cu} \\
\mathrm{Zn} \\
\mathrm{Ni} \\
\mathrm{Cr}\end{array}$ & 1.0 & $\begin{array}{l}0.42 \\
1.0\end{array}$ & $\begin{array}{l}0.06 \\
0.58 \\
1.0\end{array}$ & $\begin{array}{l}0.05 \\
0.00 \\
0.41 \\
1.0\end{array}$ & $\begin{array}{r}0.59 \\
0.29 \\
0.18 \\
-0.08 \\
1.0\end{array}$ & $\begin{array}{r}-0.27 \\
0.39 \\
0.46 \\
-0.27 \\
0.16 \\
1.0\end{array}$ & $\begin{array}{c}-0.09 \\
-0.31 \\
-0.46 \\
-0.59 \\
-0.35 \\
-0.40 \\
1.0\end{array}$ & $\begin{array}{c}0.11 \\
0.57 \\
0.77 \\
0.69 \\
0.17 \\
0.30 \\
-0.75 \\
1.0\end{array}$ & $\begin{array}{c}0.20 \\
0.43 \\
0.32 \\
0.67 \\
0.10 \\
0.05 \\
-0.83 \\
0.75 \\
1.0\end{array}$ & $\begin{array}{r}0.54 \\
0.49 \\
0.14 \\
0.18 \\
0.81 \\
0.03 \\
-0.51 \\
0.34 \\
0.47 \\
1.0\end{array}$ & $\begin{array}{r}0.68 \\
0.55 \\
0.05 \\
-0.05 \\
0.70 \\
0.15 \\
-0.47 \\
0.20 \\
0.48 \\
0.85 \\
1.0\end{array}$ & $\begin{array}{r}16.54 \\
0.68 \\
4.47 \\
0.09 \\
0.45 \\
1.69 \\
1.07 \\
50.00 \\
87.00 \\
148.00 \\
164.00\end{array}$ & $\begin{array}{r}3.69 \\
0.10 \\
0.18 \\
0.01 \\
0.02 \\
0.09 \\
0.20 \\
3.57 \\
5.76 \\
25.23 \\
29.02\end{array}$ \\
\hline \multicolumn{14}{|c|}{ Unit 4-5 (Riss-Würm) } \\
\hline $\begin{array}{l}\mathrm{CaCO}_{3} \\
\mathrm{C}_{\text {org }} \\
\mathrm{Fe} \\
\mathrm{Mn} \\
\mathrm{Ti} \\
\mathrm{K} \\
\mathrm{Na} \\
\mathrm{Cu} \\
\mathrm{Zn} \\
\mathrm{Ni} \\
\mathrm{Cr}\end{array}$ & 1.0 & $\begin{array}{c}-0.03 \\
1.0\end{array}$ & $\begin{array}{l}0.45 \\
0.47 \\
1.0\end{array}$ & $\begin{array}{l}0.24 \\
0.03 \\
0.29 \\
1.0\end{array}$ & $\begin{array}{r}0.25 \\
-0.07 \\
0.69 \\
0.51 \\
1.0\end{array}$ & $\begin{array}{r}-0.31 \\
0.31 \\
0.26 \\
0.07 \\
0.37 \\
1.0\end{array}$ & $\begin{array}{c}-0.59 \\
-0.30 \\
-0.65 \\
-0.08 \\
-0.50 \\
-0.32 \\
1.0\end{array}$ & $\begin{array}{c}0.54 \\
0.63 \\
0.72 \\
0.47 \\
0.30 \\
0.17 \\
-0.50 \\
1.0\end{array}$ & $\begin{array}{r}0.08 \\
0.85 \\
0.54 \\
0.35 \\
0.17 \\
0.35 \\
-0.36 \\
0.81 \\
1.0\end{array}$ & $\begin{array}{r}0.77 \\
0.13 \\
0.64 \\
0.46 \\
0.59 \\
-0.07 \\
-0.70 \\
0.55 \\
0.30 \\
1.0\end{array}$ & $\begin{array}{r}0.74 \\
0.28 \\
0.64 \\
0.47 \\
0.56 \\
0.18 \\
-0.75 \\
0.70 \\
0.49 \\
0.89 \\
1.0\end{array}$ & $\begin{array}{r}13.50 \\
0.95 \\
4.09 \\
0.10 \\
0.41 \\
1.67 \\
1.31 \\
53.00 \\
87.00 \\
112.0 \\
112.0\end{array}$ & $\begin{array}{r}4.91 \\
0.51 \\
0.40 \\
0.05 \\
0.04 \\
0.10 \\
0.35 \\
11.41 \\
12.13 \\
29.53 \\
29.77\end{array}$ \\
\hline
\end{tabular}

and Neoeuxinic deposits is approximately an order of magnitude lower than that of Recent and old Black Sea sediments.

This impoverishment of sediments in Mo and Se observed in Neoeuxinic deposits is linked with the absence of $\mathrm{H}_{2} \mathrm{~S}$ in the lowermost water column, as well as with the penetration of Mediterranean waters, an important source for some microelements, into the Black Sea basin.

The above observations indicate that $\mathrm{Mo}$ and $\mathrm{Se}$ accumulation at Site 380 took place primarily under conditions analogous to those of the Neoeuxinic basin, i.e., under conditions of relatively high sedimentation rates, low content of organic matter in the sediments, relatively weak, but often episodic, development of reducing conditions, low salinity, and oxidizing conditions of near bottom waters.
That correlation of Se with Mo is observed in the core only at specific horizons, points to the weak development of the Se concentration processes by $\mathrm{Mn}$. The correlation of Se with $\mathrm{C}_{\text {org }}$ is more often the fact. It is known that organic matter may directly service as an accumulator of $\mathrm{Se}$, but organic matter favors the formation of authigenic sulfides by reduction and $\mathrm{Se}$ associated with these sulfides. The mechanism of the process enabling Se to accumulate in reduced sediments is $\mathrm{Se}$ reduction $\left(\mathrm{SeO}_{4}\right)^{2}-$ to $\left(\mathrm{SeO}_{3}\right)^{2}$-and then to elementary $\mathrm{Se}^{0}$. Elemental Se is taken into the lattice of the Fe sulfides, commonly as an isomorphous substitution for sulfur.

In the Black Sea during the post-Neoeuxinic period (Holocene), the Mediterranean waters became an additional source of Mo and some other elements. The balance calculations of Mo in the Black Sea (according to the water balance of the basin) show that the lower 

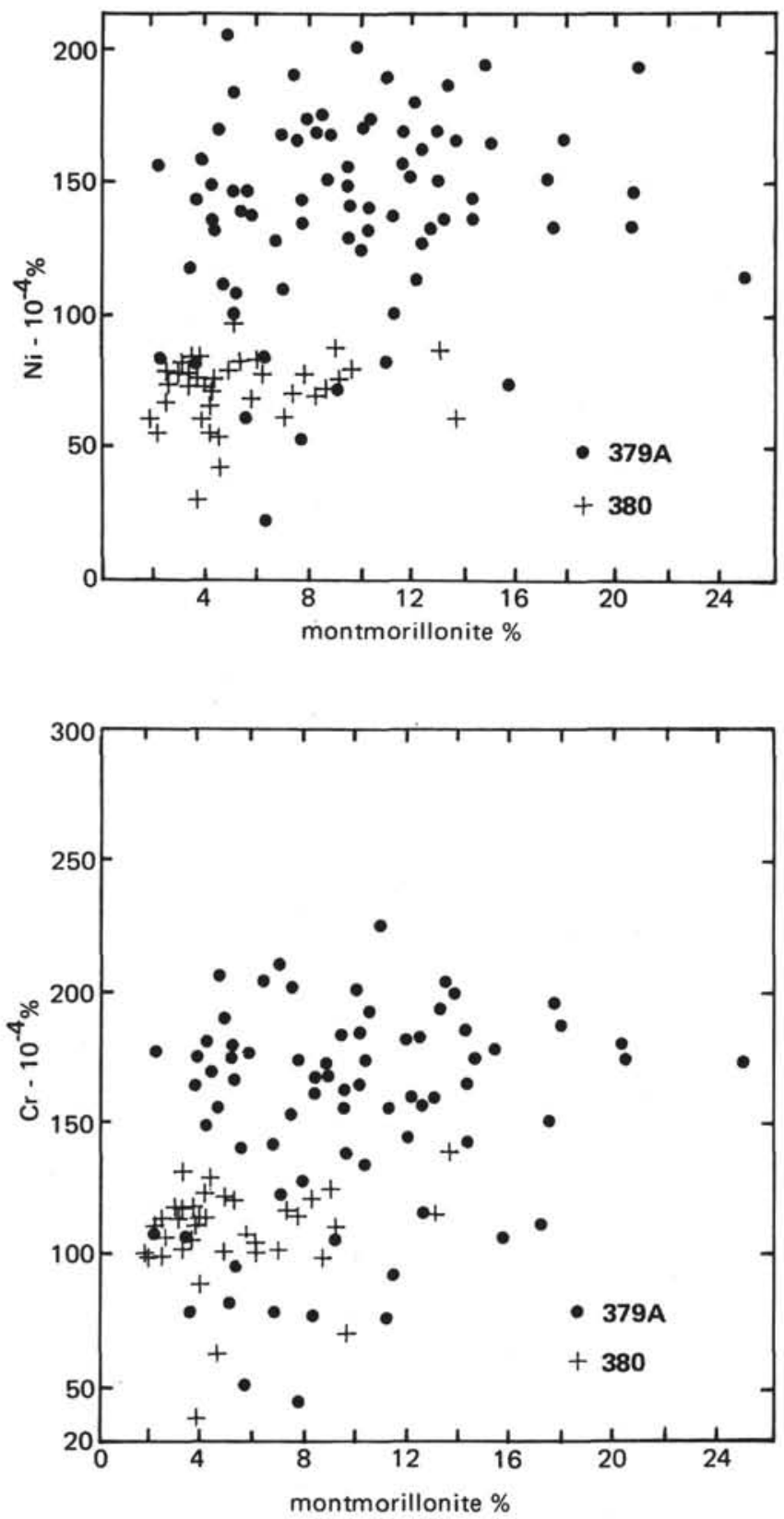

Figure 26. Ni and $\mathrm{Cr}$ distribution in Holes $379 \mathrm{~A}$ and 380 as a function of montmorillonite contents.

Bosporus current was of primary importance, supplying $80 \%$ of the total mass of Mo transported into the sea. Since the total Mo input amounts to $2061 \mathrm{t} / \mathrm{yr}$, and outflow up to $1090 \mathrm{t} / \mathrm{yr}$, annually a net Mo concentration of $\sim 1000 \mathrm{t}$ occurs in the reduced sediments.

It is due to the lower Bosporus current that the Mo concentration in Recent and old Black Sea deposits is by one order more (and sometimes by two) in excess of its clarke in sedimentary rocks.

\section{Group of Hydrolysate Elements (Ti, Sn,}

$\mathrm{Hf}, \mathrm{Ta}, \mathrm{Th}$ ) and $\mathrm{Cr}, \mathrm{V}$

This group of elements is made of typical hydrolysates, as well as of low mobility $\mathrm{Cr}$ and $\mathrm{V}$.

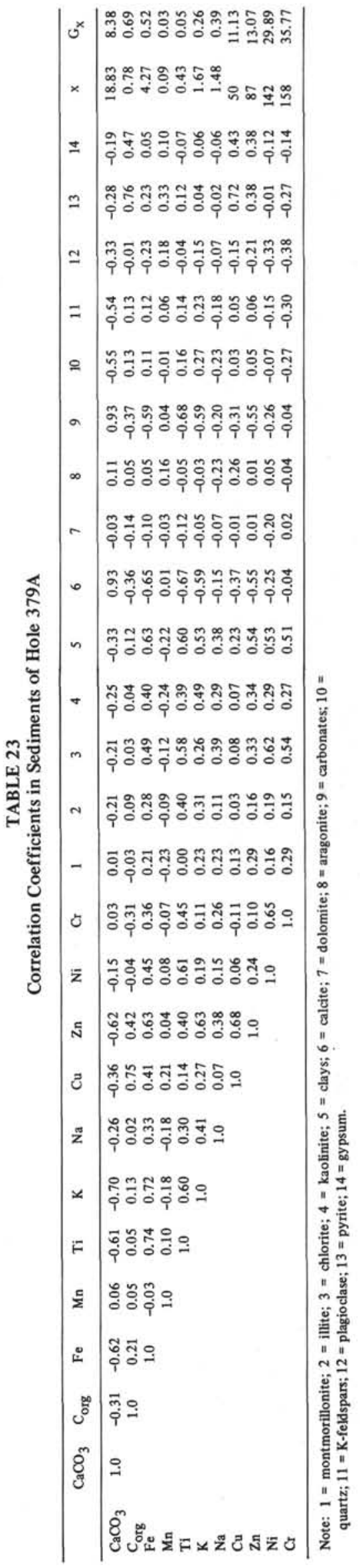



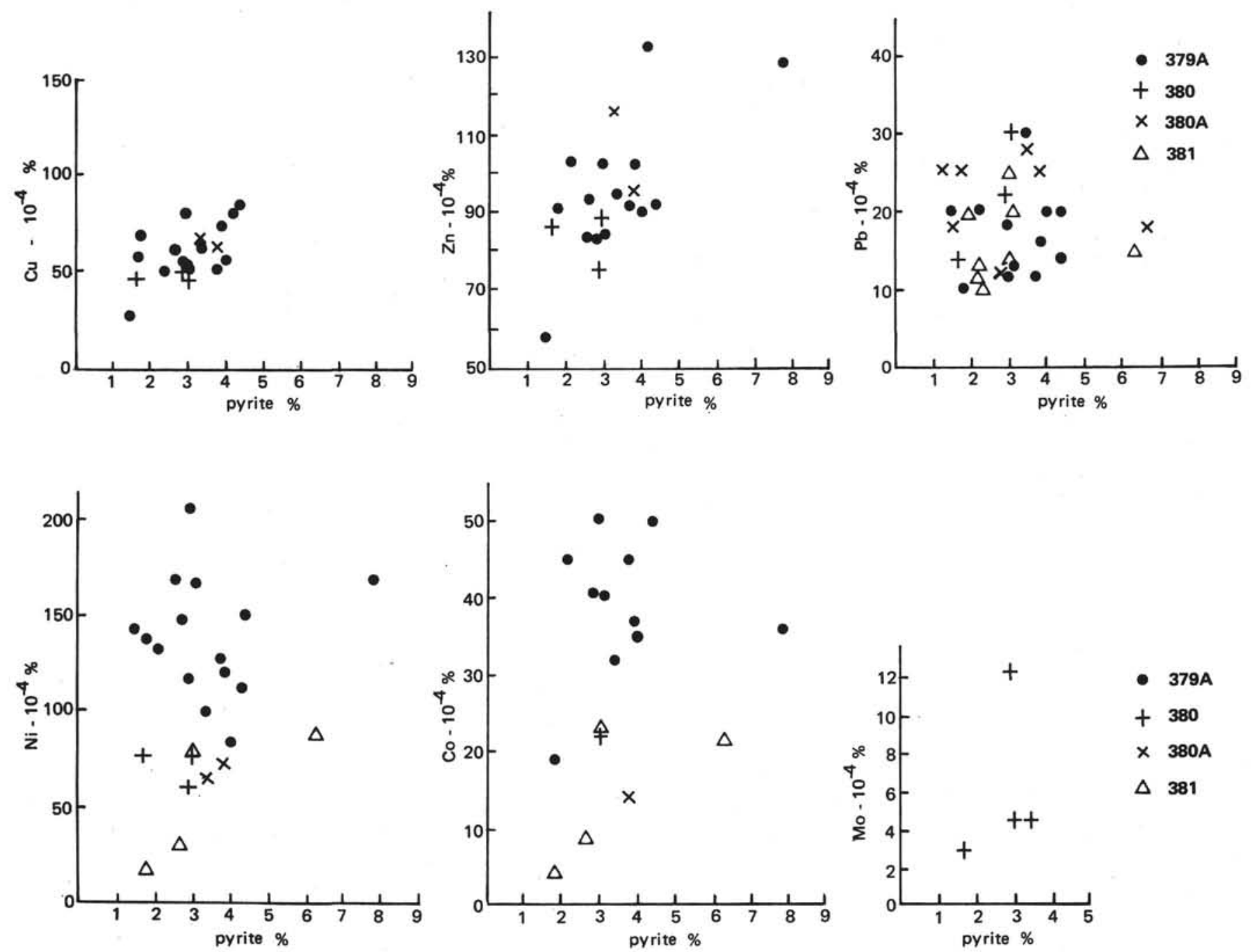

Figure 27. Correlation between micro-elements and pyrite in site sediments.

The geochemistry of $\mathrm{Ti}, \mathrm{Cr}, \mathrm{V}$, and $\mathrm{Th}$ in the Recent Black Sea sediments is rather well known (Glagoleva, 1959, 1961; Starich et al., 1961a, b; Strakhov, 1961; Kuznetsov, 1971; Strakhov et al., 1971); data on Sn, Hf, and $\mathrm{Ta}$ have been obtained here for the first time.

Maximum and high contents of $\mathrm{Ti}, \mathrm{Cr}$, and $\mathrm{V}$ in the Recent sediments have been found in the peripheral zones of the basin where basic rock weathering products are supplied by Pontic drainage area and where localized high contents of montmorillonite occur. $\mathrm{Cr}$ has close correlation with montmorillonite; $\mathrm{Ti}$ has the least. In hydrodynamically calm regions (halistatic), sediments are generally impoverished in these elements, but significant amounts of them do occur in certain parts of the calm zones, and are concentrated in nannofossil oozes enriched in organic matter. $\mathrm{Ti}$ is consumed by phytoplankton and $\mathrm{Cr}$ and $\mathrm{V}$ migrate in solution as hydroxides, becoming absorbed onto clay minerals and organic matter.

$\mathrm{Ti}, \mathrm{Cr}$, and $\mathrm{V}$ are found mainly in Recent sediments in the vicinity of Hole $379 \mathrm{~A}$, but are also significant there in late Cenozoic sediments. At Site 380 they occur in lesser quantities (Table 25, Figure 26). In both cases they decrease in quantity with increase in grain size, although $\mathrm{Ti}$ is found mostly in coarse pelitic material where $\mathrm{V}$ and $\mathrm{Cr}$ are more characteristic of the subcolloidal fraction.

\section{Tin}

In the Miocene-Quaternary sediment sections, contents of $\mathrm{Sn}$ are noticeably lower than $\mathrm{K}_{\mathrm{o}}$ and close to $\mathrm{K}_{\mathrm{a}}$, suggesting its having been derived from erosion products of acid rocks. Higher $\mathrm{Sn}$ concentrations at Site 380 (Pliocene-Quaternary section) than at Site 379 may be related to differential supply of acidic components.

The Miocene varves (Unit B-4-e, Hole 380A) are enriched in Sn, seemingly caused by an increased quantity of accessory minerals in the fine aleuritic beds of these varves.

\section{Thorium}

Study of thorium in the Black Sea bottom sediments (Starik et al., 1961; Kuznetsov, 1971) showed that $\mathrm{Th}^{232}$ was closely associated with terrigenous clastic clayey material whereas the main $\mathrm{Th}^{230}$ concentrators were found to be $\mathrm{Fe}, \mathrm{Mn}, \mathrm{C}_{\text {ors }}$, and $\mathrm{P}$. 
TABLE 24

Chemical Composition of Sediments Enriched in Organic Matter

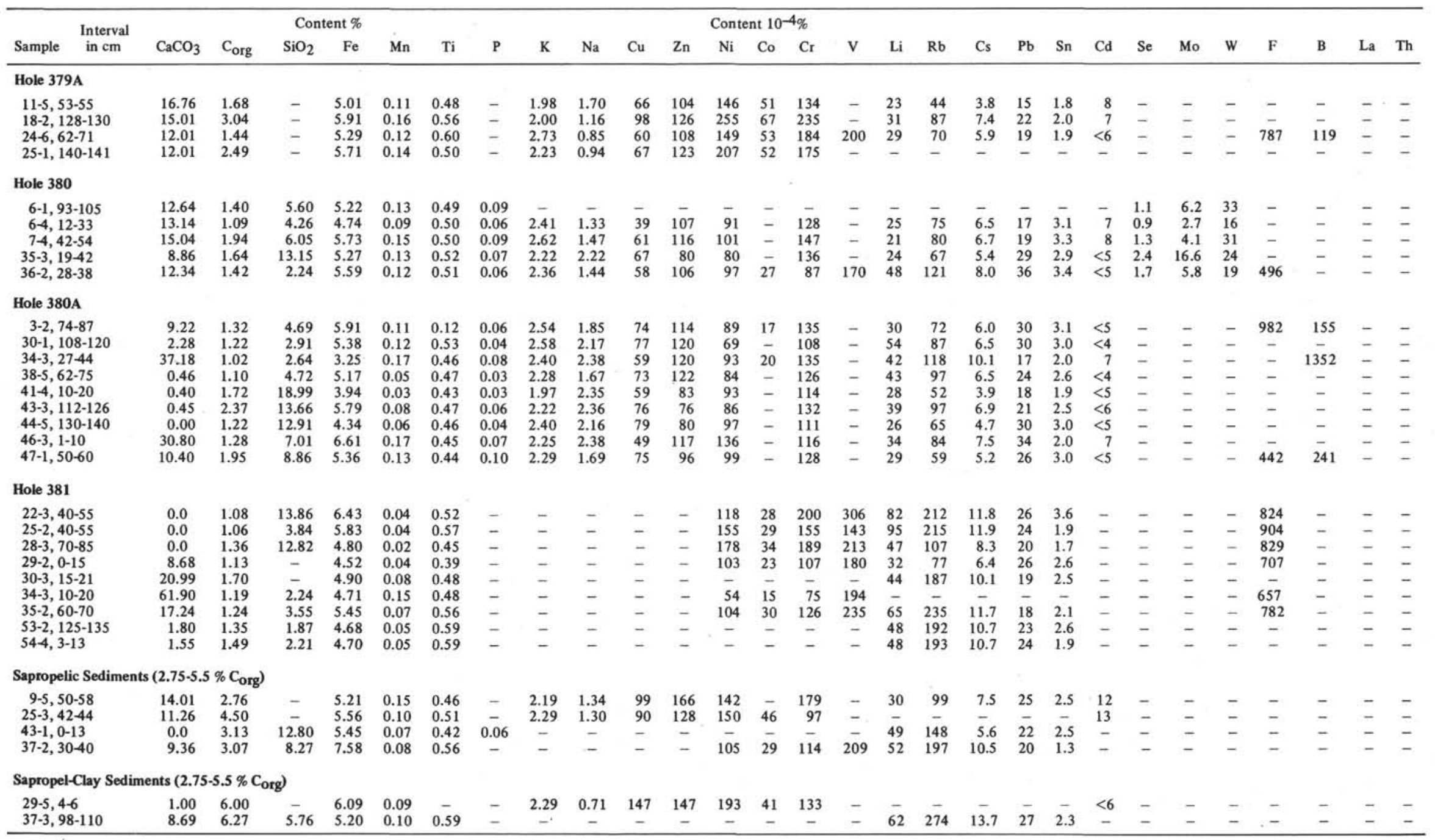



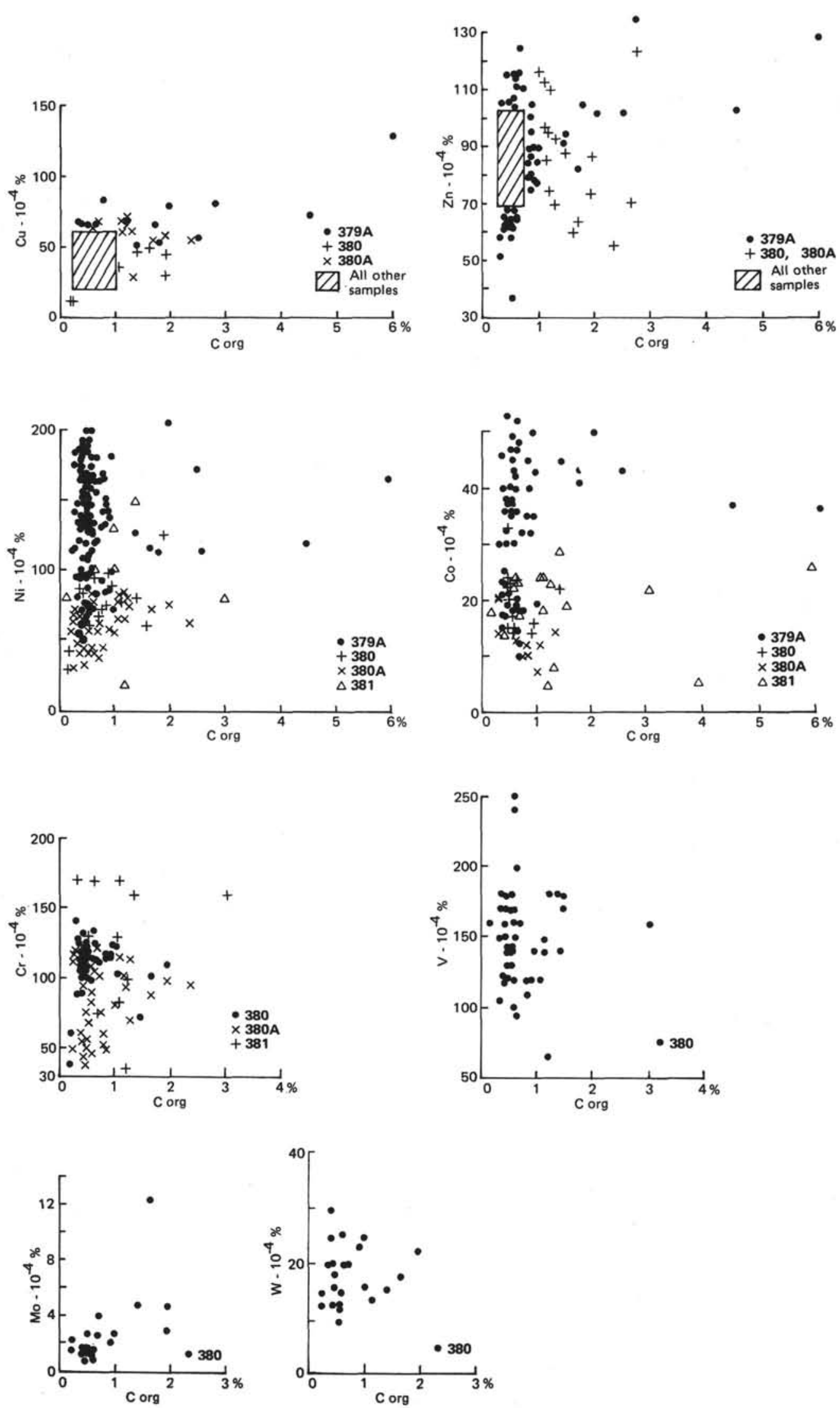

Figure 28. Correlation between micro-elements and $C_{\text {org }}$ in site sediments. 
TABLE 25

Average Contents of Hydrolysate Elements (Ti, Cr, V, Th, Sn, Hf, Ta)

\begin{tabular}{|c|c|c|c|c|c|c|c|c|c|c|c|c|c|c|c|}
\hline \multirow{3}{*}{ Hole } & & \multicolumn{2}{|c|}{ Content $(\%)$} & \multicolumn{12}{|c|}{ Content $\left(10^{-4} \%\right)$} \\
\hline & & \multicolumn{2}{|l|}{$\mathrm{Ti}$} & \multicolumn{2}{|c|}{$\mathrm{Cr}$} & \multicolumn{2}{|c|}{ V } & \multicolumn{2}{|c|}{$\mathrm{Sn}$} & \multicolumn{2}{|c|}{ Th } & \multicolumn{2}{|c|}{ Hf } & \multicolumn{2}{|c|}{$\mathrm{Ta}$} \\
\hline & & 1 & 2 & 1 & 2 & 1 & 2 & 1 & 2 & 1 & 2 & 1 & 2 & 1 & 2 \\
\hline \multirow[b]{2}{*}{$379 A$} & a & $0.28-0.54$ & 0.42 & -301 & 152 & $5-240$ & 145 & $1.0-2.5$ & 1. & $2-10$ & 8 & $0.6-8.0$ & 2.9 & $0.2-2.4$ & 1.1 \\
\hline & $\mathrm{b}$ & & - & $57-288$ & - & - & - & - & - & $3-13$ & 9 & 10.0 & 3.6 & -3.1 & 1.3 \\
\hline \multirow{2}{*}{380} & a & $0.15-0.57$ & 0.46 & $28-140$ & 100 & $110-180$ & 160 & $1.0-2.8$ & 2.2 & $2-12$ & 7 & -21.7 & 4.7 & 2.5 & 1.5 \\
\hline & b & $0.26-0.67$ & 0.54 & $49-162$ & 117 & $124-204$ & 162 & $1.6-3.4$ & 2.4 & $2-13$ & 8 & 5.5 & 5.4 & 2.9 & 1.7 \\
\hline \multirow{2}{*}{$380 \mathrm{~A}$} & a & $0.07-0.55$ & 0.35 & $38-128$ & 97 & - & - & $1.0-4.0$ & 1.6 & $1-13$ & 6 & 4.7 & 2.3 & 2.9 & 0.8 \\
\hline & b & $0.07-0.69$ & 0.49 & $89-154$ & 137 & - & - & $1.3-6.5$ & 2.3 & $1-17$ & 8 & 6.3 & 3.0 & $0.3-2.9$ & 1.0 \\
\hline \multirow{2}{*}{381} & a & $0.03-0.55$ & 0.36 & $25-210$ & 110 & $65-250$ & 147 & $1.0-2.5$ & 1.7 & $4-17$ & 11 & $0.5-9.7$ & 3.1 & $0.1-1.4$ & 0.6 \\
\hline & b & $0.04-0.97$ & 0.48 & $46-221$ & 146 & $143-306$ & 195 & $1.1-3.6$ & 2.2 & $7-19$ & 15 & $0.5-9.7$ & 3.6 & $0.2-1.4$ & 0.7 \\
\hline \multirow[t]{2}{*}{ All holes } & a & $0.03-0.57$ & - & $25-301$ & - & $65-250$ & - & $1.0-4.0$ & - & $1-17$ & 8 & $0.1-21.7$ & 3.0 & $0.1-2.9$ & 1.0 \\
\hline & b & $0.04-0.97$ & - & $46-221$ & - & $124-306$ & - & $1.1-6.5$ & - & $1-19$ & 10 & $0.1-25.5$ & 3.7 & $0.2-3.1$ & 1.2 \\
\hline
\end{tabular}

Note: $a=$ natural sediment $; \mathrm{b}=$ on a clastic matter basis (the coefficient 1.6 for $\mathrm{SiO}_{2 \text { amorph }}$ was not used at recalculation of Th, $\mathrm{Hf}$, Ta); 1 = range; 2 = average.

Thorium content ranges from 1 to $19 \mathrm{ppm}$ with average values from 6 (Hole 380A) to $11 \mathrm{ppm}$ (Site 381). Cyclically insignificant changes in $\mathrm{Th}$ concentrations were observed in Holes 379A, 380, and $380 \mathrm{~A}$, being lower during the Glacial and higher during the Interglacial epochs. Since Th is a typical hydrolysate and accumulates in the fine fraction, it preferentially accumulates during temperate periods when mainly fine clayey material is transported into the sea.

\section{Hafnium}

Hafnium contents range from 0.1 to $21.7 \mathrm{ppm}$; average values for all sites is equal 2.3-4.7 (Table 25). These values are higher than those for magmatic rocks but lower than $\mathrm{K}_{\mathrm{o}}$. When biogenic material is excepted, average concentrations reach 3-5.4.

The average values by units show that in the lower part of Hole 379A, before R-W, a cyclic recurrence is observed which is inverse to the thorium distribution. During the Interglacial epochs $\mathrm{Hf}$ contents are lower than those during the Glacial ones. The same is true of Holes $380 / 380 \mathrm{~A}$.

\section{Tantalum}

Average Ta contents are lower than $\mathrm{K}_{\mathrm{o}}$ (Tables 3, 9 , and 25). In sediments of late Glacial units its concentrations are high; older sediments (Eopleistocene) are characterized by sharp decline of $\mathrm{Ta}$ concentrations, increasing again in Pliocene sediments.

\section{Alkaline Metals (K, Na, Li, Rb, Cs)}

Alkaline metals have an affinity for finely dispersed terrigenous material, mainly with clay minerals and, to a lesser extent, micas and feldspars. Because $\mathrm{K}, \mathrm{Li}, \mathrm{Rb}$, and $\mathrm{Cs}$ in the sedimentary cycle are primarily associated with clay minerals (Boyko, 1964, p. 400), we believe that these elements are mainly supplied to the sea along with fine-suspended matter. The extraction of these elements from sea water is insignificant, if it occurs at all (Boyko, 1964, p. 405), and $\mathrm{Li}, \mathrm{Rb}$, and $\mathrm{Cs}$ geochemically follow $\mathrm{K}$ and $\mathrm{Na}$.

The geochemistry of alkaline metals in the Black Sea bottom sediments is poorly known, although data on
$\mathrm{K}, \mathrm{Na}, \mathrm{Li}$, and $\mathrm{Rb}$ distributions in the upper Quaternary sediment sections have recently been obtained (Hirst, 1974).

In the drilled sediments, the alkaline suite present in the detrital clay minerals is shown in correlation charts (Figure 29). Their quantitative distributions (Table 26) in the sections is controlled by terrigenous material distribution, which is rather changeable in content and composition (Figures 3, 9, 11, and 13).

However each element does have its own specific geochemical behavior clearly expressed: (1) by weak direct correlations between certain elements ( $\mathrm{K}$ and $\mathrm{Na}$ in particular; Tables 22, 23); (2) by observation of maximum and minimum contents in different units. Thus, Holes 380 and $380 \mathrm{~A}$ maximum $\mathrm{K}$ contents occur in Unit B-1-h, maximum Na content occurs in Unit B2, and maxima of $\mathrm{Li}, \mathrm{Rb}$, and $\mathrm{Cs}$ occur in Pleistocene sediments (Unit B-1-h); (3) $\mathrm{K}$ and Na contents in Holes $379 \mathrm{~A}$ and 380 are almost the same, where $\mathrm{Li}, \mathrm{Rb}$, and Cs are more abundant at Site 380 than at Site 379; and (4) average $K$ content, as a rule, is higher than its $K_{0}$, that of $\mathrm{Na}$ is close to $\mathrm{K}_{\mathrm{o}}$; those of $\mathrm{Li}, \mathrm{Rb}$, and $\mathrm{Cs}$ are significantly lower than their $\mathrm{K}_{\mathrm{o}}$, approaching the clarke of intermediate and acid rocks.

In general, rather high $\mathrm{Li}, \mathrm{Rb}$, and $\mathrm{Cs}$ contents are found in low siliceous diatomaceous oozes and siderite beds from the upper Pliocene sediments. This is probably due to source material. According to Lubchenko and Belova's (1973) data, the finest suspended matter of the Danube and the Dnieper now consists mainly of those mixed-layered (micamontmorillonite) minerals which are concentrators of Li, Rb, and Cs (Boyko, 1964, p. 400). Palynological data indicate a warm humid climate during Pliocene, a time when chemical weathering processes in the drainage area were more intensive than in Recent time.

\section{Phosphorus, Fluorine, and Boron}

\section{Phosphorus}

In Recent sediments, phosphorus, as well as other mobile elements, is characterized by markedly high pelagic maximum contents: $>0.05 \%$ in the natural sediments and $>0.10 \%$ after conversion (Glagoleva, 1961; Strakhov et al., 1971). In Holes 380/380A its contents range from 0.01 to $0.14 \%$, or converted, from 

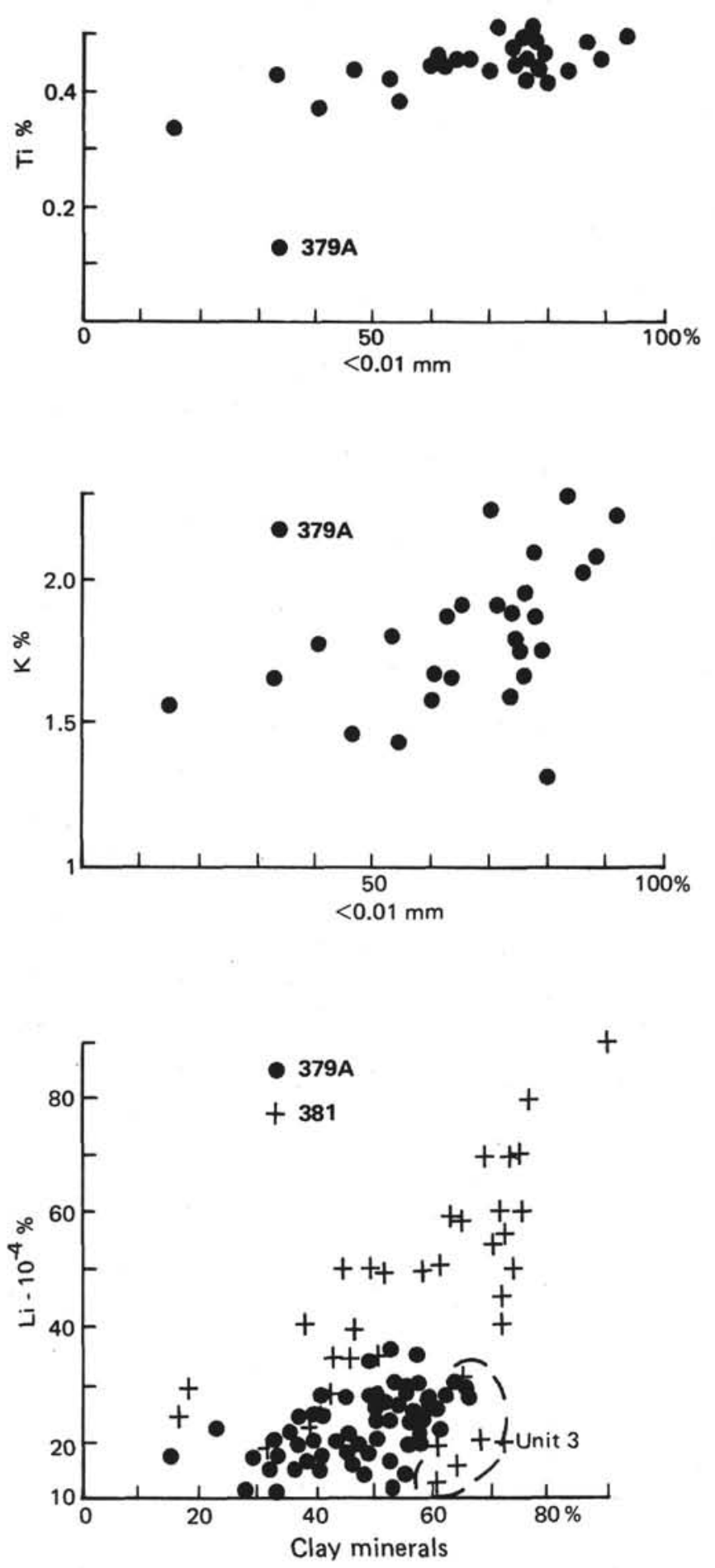

Figure 29. Correlation between $T i$ and $K$ and the $<0.01$ $\mathrm{mm}$ fraction and between $\mathrm{Li}$ and total clay mineral contents in sediments.

0.02 to $0.33 \%$ (Table 27 ), thus reaching the maximum values observed in Recent sediments.

Based on analyses of 1000 Atlantic Ocean sediment samples, phosphorus is closely associated with the fine fraction of terrigenous sediments, i.e., a direct correlation exists between $P$ and the $<0.01 \mathrm{~mm}$ fraction, a correlation generally observed at all sites.

\section{Fluorine}

Fluorine content in Holes 380 and 380A range from 80 to $2400 \mathrm{ppm}$ (Table 27). Average fluorine content for the three sites is $530 \mathrm{ppm}$. This value is similar to the average estimate $(540 \mathrm{ppm})$ obtained by Shishkina $(1959,1972)$ for the surface sediments of the Black Sea. Maximum concentrations of fluorine (Holes 380 and 380A) are in Eopleistocene sediments, represented by clays and terrigenous, low-carbonate oozes with a dolomite admixture (Figure 30). Fluorine increases (slightly to $560 \mathrm{ppm}$ ) in low-siliceous terrigenous muds deposited during the Riss-Würm Interglacial period (Unit B-1-d).

At Site 381 a high fluorine content (Table 16) is found in sediments between 160 and 260 meters, of Eopleistocene and late Pliocene age. Relatively large amounts of fluorine $(640 \mathrm{ppm})$ occur in black shales of Unit 9.

The lower $(\mathrm{M})$ and the middle $(\mathrm{R})$ parts of the section at Site 379 have higher fluorine contents than the upper section, which was deposited during the Würm and post-Würm periods (Figure 31). The maximum fluorine concentration at Site $379(920 \mathrm{ppm})$ was detected in terrigenous low-calcareous muds and turbidite samples from about 300 meters depth.

\section{Boron}

According to Sukhorukov and Emelyanov (1969), the average B content in modern Black Sea oozes is 116 ppm (ranging between 3 and $200 \mathrm{ppm}$ ). In biogenic calcareous sands and coarse aleurites, the average B content is lower, $95 \mathrm{ppm}$. Boron content is much higher in the eastern Black Sea than in the western part, most likely as a result of supply from mud volcanoes which are rather active on the Kerch and Taman peninsulas (Sukhorukov and Emelyanov, 1969).

Average boron content in the bottom sediments (to a depth of $4 \mathrm{~m}$ ) of the Black Sea was determined by Gursky (1971) to be $108 \pm 3 \mathrm{ppm}$ (476 analyses) and 114 ppm (ranging between 41 and 390) by Hirst (1974); in the sediments we analyzed from the drill sites, B ranges from 111 to $147 \mathrm{ppm}$ (Table 27). Boron contents in Hole $380 / 380 \mathrm{~A}$ sediments ranges considerably. When analyzing curves of B distribution in sediments and in the $<0.001 \mathrm{~mm}$ fraction, as well as the amounts of an "equivalent boron," 2 for Holes 380/380A, trends are seen which may be associated with changes of salinity in the paleobasin (Figure 30).

Minimum values of these elements, $20 \mathrm{ppm} \mathrm{Pb}, 100$ $200 \mathrm{ppm} \mathrm{Zn}$, occur in Recent sediments in the vicinity of Site 379. They are approximately the same in the Quaternary sediment section (Table 28).

Recent sediments in the vicinity of Sites 380 and 381 are impoverished in $\mathrm{Zn}$ (100-200 ppm) and enriched in $\mathrm{Pb}(40-60 \mathrm{ppm}) . \mathrm{Zn}$ content in the studied sediment section is comparable to the minimum values in Recent sediments. $\mathrm{Pb}$ contents in sediments at Site 380 are similar to those in Recent muds in this area (20-40

\footnotetext{
${ }^{2}$ Equivalent boron $=(\mathrm{B}$ content $[\%] 8.5) /\left(\mathrm{K}_{2} \mathrm{O}\right.$ content [\%] $)$ (Walker and Price, 1963). This indicator may be used as an indicator of illite content.
} 
TABLE 26

Average Contents of Alkaline Elements (K, Na, Li, Rb, Cs)

\begin{tabular}{|c|c|c|c|c|c|c|c|c|c|c|c|}
\hline \multirow{3}{*}{ Hole } & & \multicolumn{4}{|c|}{ Content (\%) } & \multicolumn{6}{|c|}{ Content $\left(10^{-4} \%\right)$} \\
\hline & & \multicolumn{2}{|c|}{$\mathrm{K}$} & \multicolumn{2}{|c|}{$\mathrm{Na}$} & \multicolumn{2}{|c|}{$\mathrm{Li}$} & \multicolumn{2}{|c|}{$\mathrm{Rb}$} & \multicolumn{2}{|c|}{ Cs } \\
\hline & & 1 & 2 & 1 & 2 & 1 & 2 & 1 & 2 & 1 & 2 \\
\hline \multirow{2}{*}{$379 \mathrm{~A}$} & a & $0.58-2.92$ & 1.68 & $0.17-1.74$ & 0.91 & $8-35$ & 22 & $22-90$ & 54 & $2.0-6.8$ & 4.7 \\
\hline & b & $1.11-3.17$ & - & $0.20-1.92$ & - & - & - & - & - & - & - \\
\hline \multirow{2}{*}{380} & a & $1.09-2.00$ & 2.09 & $0.76-1.66$ & 1.12 & $10-55$ & 32 & $30-150$ & 76 & $35-7.0$ & 5.6 \\
\hline & $\mathrm{b}$ & $1.83-3.26$ & 2.44 & $0.90-2.22$ & 1.31 & $14-63$ & 37 & $47-183$ & 89 & $5.6-9.5$ & 6.5 \\
\hline \multirow{2}{*}{$380 \mathrm{~A}$} & a & $1.16-2.91$ & 1.88 & $0.89-2.07$ & 1.07 & $10-60$ & 30 & $30-120$ & 63 & $2.5-7.0$ & 4.7 \\
\hline & $\mathrm{b}$ & $1.93-6.21$ & 2.44 & $1.13-4.15$ & 1.51 & $20-72$ & 42 & $52-227$ & 89 & $3.9-13.9$ & 6.6 \\
\hline \multirow{2}{*}{381} & a & - & - & - & - & $12-90$ & 44 & $60-200$ & 124 & $3.0-12.0$ & 8.0 \\
\hline & $\mathrm{b}$ & - & - & - & - & $12-101$ & 58 & $62-274$ & 164 & $3.1-16.9$ & 1.1 \\
\hline \multirow{2}{*}{ All holes } & a & $0.58-2.92$ & - & $0.17-2.07$ & - & $8-90$ & - & $22-200$ & - & $2.0-12.0$ & - \\
\hline & $\mathrm{b}$ & $1.83-6.21$ & - & $0.90-4.15$ & - & $12-101$ & - & $47-274$ & - & $3.1-16.9$ & - \\
\hline
\end{tabular}

Note: $a=$ natural sediment $; \mathrm{b}=$ on $\mathrm{a}$ carbonate free basis; $1=$ range $; 2=$ average.

TABLE 27

Average Contents of $\mathrm{P}, \mathrm{F}$, and $\mathrm{B}$

\begin{tabular}{|c|c|c|c|c|c|c|c|}
\hline \multirow[b]{2}{*}{ Hole } & & \multicolumn{2}{|c|}{ Content (\%) } & \multicolumn{4}{|c|}{ Content $\left(10^{-4} \%\right)$} \\
\hline & & $1 P$ & 2 & $\mathrm{~F}$ & 2 & $1 \mathrm{~B}$ & 2 \\
\hline \multirow[t]{2}{*}{$379 \mathrm{~A}$} & a & - & - & $250-2400$ & 590 & $42-154$ & 111 \\
\hline & $\mathrm{b}$ & - & - & - & - & - & - \\
\hline \multirow{2}{*}{380} & a & $0.03-0.09$ & 0.06 & $370-610$ & 483 & $128-161$ & 147 \\
\hline & $\mathrm{b}$ & $0.05-0.11$ & 0.07 & $444-722$ & 563 & $144-186$ & 171 \\
\hline \multirow[t]{2}{*}{$380 \mathrm{~A}$} & a & $0.01-0.14$ & 0.05 & $80-860$ & 636 & $66-223$ & 125 \\
\hline & $\mathrm{b}$ & $0.02-0.33$ & 0.07 & $138-2373$ & 896 & $69-355$ & 176 \\
\hline \multirow[t]{2}{*}{381} & a & - & - & $220-760$ & 526 & - & - \\
\hline & $\mathrm{b}$ & - & - & $346-1324$ & 696 & - & - \\
\hline \multirow[t]{2}{*}{ All holes } & a & $0.01-0.14$ & - & $80-2400$ & - & $42-223$ & - \\
\hline & b & $0.02-0.33$ & - & $138-2373$ & - & $69-355$ & - \\
\hline
\end{tabular}

Note: $\mathrm{a}=$ natural sediment $\mathrm{b}=$ on a clastic matter basis; 1 = range $; 2$ = average.

TABLE 28

Average Contents of $\left(10^{-4} \%\right)$ Chalcophile Group Elements $(\mathrm{Zn}, \mathrm{Pb})$

\begin{tabular}{|c|c|c|c|c|c|}
\hline \multirow[b]{2}{*}{ Hole } & & \multicolumn{2}{|c|}{$\mathrm{Zn}$} & \multicolumn{2}{|c|}{$\mathrm{Pb}$} \\
\hline & & 1 & 2 & 1 & 2 \\
\hline \multirow[t]{2}{*}{$379 \mathrm{~A}$} & a & $37-134$ & 88 & $3-30$ & 15 \\
\hline & b & $73-158$ & - & - & - \\
\hline \multirow[t]{2}{*}{380} & $\mathrm{a}$ & $37-117$ & 89 & $6-25$ & 18 \\
\hline & b & $65-130$ & 104 & $11-33$ & 21 \\
\hline \multirow{2}{*}{$380 \mathrm{~A}$} & a & $40-97$ & 79 & $4-30$ & 16 \\
\hline & b & $76-170$ & 111 & 8-75 & 23 \\
\hline \multirow[t]{2}{*}{381} & a & - & - & $3-30$ & 16 \\
\hline & b & - & - & $6-35$ & 21 \\
\hline \multirow[t]{2}{*}{ All holes } & $\mathrm{a}$ & $40-134$ & - & $3-30$ & - \\
\hline & b & $65-170$ & - & $6-75$ & - \\
\hline
\end{tabular}

Note: $\mathrm{a}=$ natural sediment $; \mathrm{b}=$ on $\mathrm{a}$ clastic matter basis; 1 = range; 2 = average.

ppm), reaching the upper limit (50 ppm) only in single beds which occur most commonly in the Interglacial series of Quaternary sediments.

In the Hole 379A Quaternary sediments, the $\mathrm{Pb}$ content is either on the order of the minimum values in Recent sediments $(<20 \mathrm{ppm})$, or exceeds these $(20-40$ $\mathrm{ppm}$ ); the higher values occur in discrete beds within the Glacial and, more often, Interglacial units. Values as high as the maximum $\mathrm{Pb}$ contents of Recent sediments were not found.
Relatively increased $\mathrm{Pb}$ contents occur in both muds and low-calcareous muds with low organic matter content. No definite correlation exists between $\mathrm{Pb}$ and $\mathrm{C}_{\text {org }}$ or sulfides (Figure 27). Thus the enrichment in $\mathrm{Pb}$ of some beds is probably due to periodically more intensive accumulation of terrigenous material enriched in $\mathrm{Pb}$. At Site 379 this material is likely derived from a Kuban source, while at Sites 380 and 381 the Danube was the probable carrier of the $\mathrm{Pb}$-rich sediments. The fact that the average $\mathrm{Pb}$ content increases in the lower Site 380 units and the upper Hole 379A units suggest a relatively more intensive Danube contribution at Site 379 during the first half of Pleistocene than during the second; mineralogical data confirm this.

$\mathrm{Zn}$ behavior in Neogene-Quaternary sediment section is somewhat different from that of $\mathrm{Pb}$. No similar localization of minimum and maximum contents exists in single beds or within major units. Average $\mathrm{Zn}$ contents by unit, for example, remain almost unchanged, whereas those of $\mathrm{Pb}$ increase at Site 380 and decrease in the Site 379 lower units. Increased $\mathrm{Zn}$ contents often occur in mud interbeds enriched in organic matter and a general correlation in quantitative $\mathrm{Zn}$ and $\mathrm{C}_{\text {org }}$ distribution is observed (Figure 28). Moreover, $\mathrm{Zn}$ is strongly dependent on the quantitative sulfide distribution (Figure 27).

Localization of certain $\mathrm{Pb}$ and $\mathrm{Zn}$ maximum concentrations is the only similarity of their distribution in Recent and older sediments. In other respects, their behavior differs considerably.

\section{CONCLUSIONS}

The study of nine macrocomponents $\left(\mathrm{CaCO}_{3}, \mathrm{C}_{\text {org }}\right.$, $\mathrm{SiO}_{2}$ amorph, $\mathrm{Fe}, \mathrm{Mn}, \mathrm{Ti}, \mathrm{P}, \mathrm{K}$, and $\mathrm{Na}$ ) and 24 minor elements (Cu, Zn, Ni, Co, Cr, V, Cd, Li, Rb, Sc, Sn, Se, Mo, W, F, B, La, Th, Hf, Eu, Sb, Ce, and Ta) in three sites revealed the following nature of their distribution in Neogene-Quaternary sediments.

1) $\mathrm{C}_{\text {org }}$ and $\mathrm{SiO}_{2}$ amorph are macrocomponents having the clearest response to changes in climatic conditions. Their abundance relatively increased in Interglacial units and decreased in Glacial units. A group of geochemically mobile elements is characterized by the same properties, and relates to the amounts of the weathered products supplied by drainage systems. The 


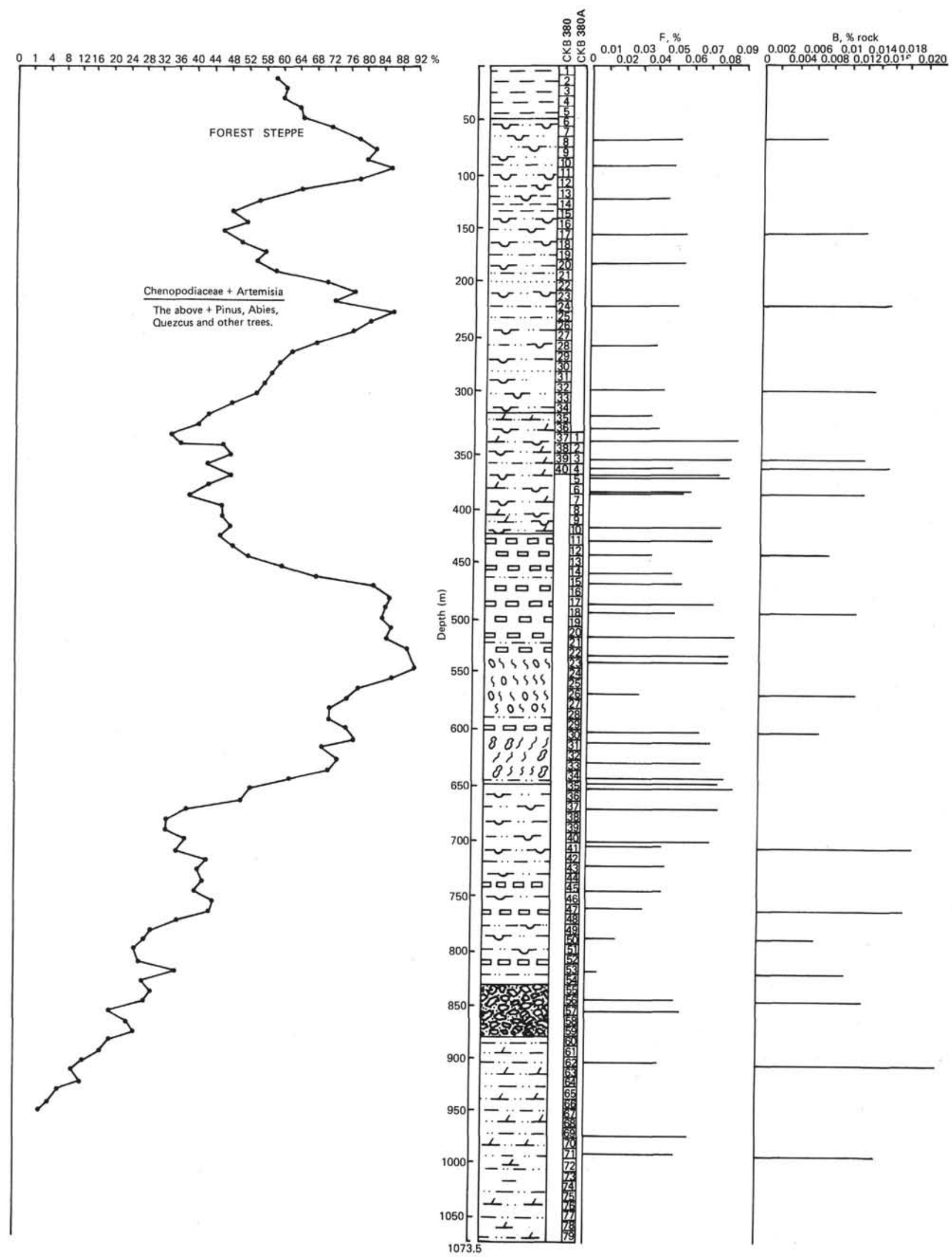

Figure 30. $F$ and $B$ distributions in Holes 380/380A sediments. 


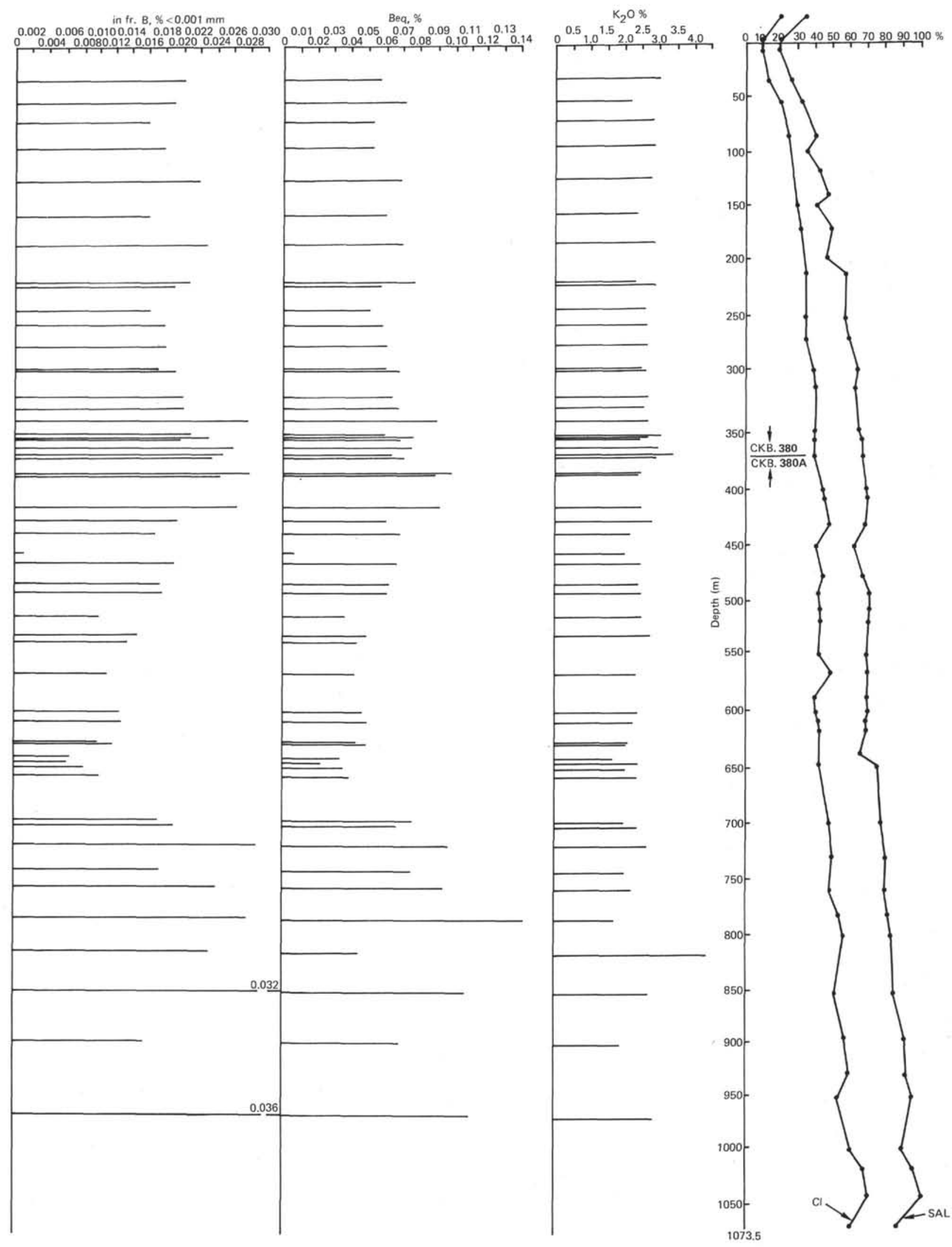

Figure 30. (Continued). 


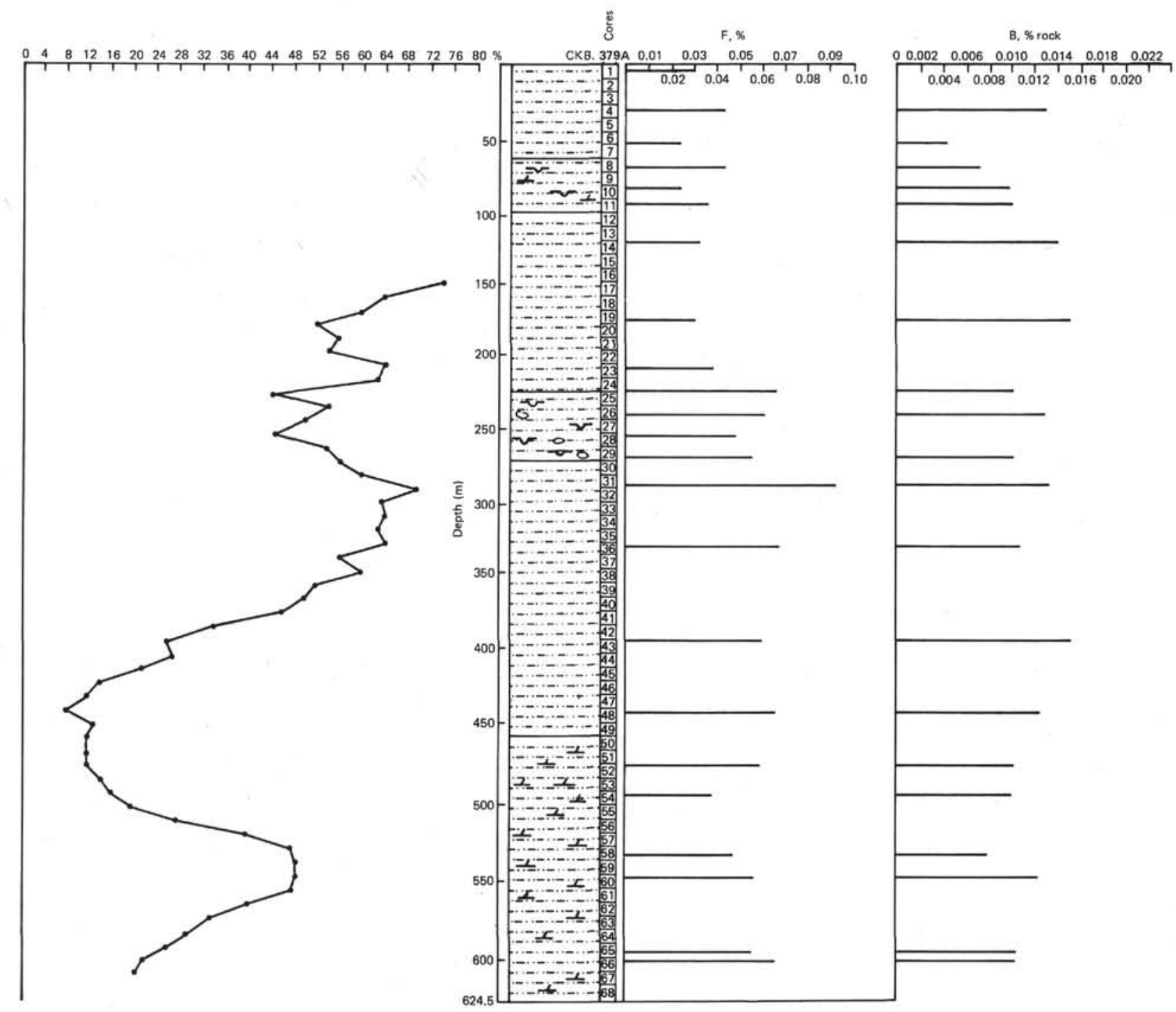

Figure 31. F and B distributions in Hole 379A sediments.

largest amounts of these products were transported during warm stages.

Increased boron contents in discrete intervals of Interglacial sediments is noted, associated with periodical increase of water salinity (inflow of Mediterranean waters).

2) The distribution of most elements in the Interglacial units is similar to that in the Holocene; the Glacial ones are similar to upper Würm units (early and middle Neoeuxinic). The individual distribution of each element is determined by mineral composition of the source areas and by mode of transport in rivers and seas. The most geochmeically mobile elements, at all stages of mid-Pleistocene period, is concentrated in pelagic, hydrodynamically static (the so-called chalistatic) areas.

A complex pattern of distribution of most minor elements in the Interglacial series is seen both in nonuniform composition and the unequal distribution of some macrocomponents with which they are closely connected ( $\mathrm{C}$ org, $\mathrm{SiO}_{2}$ amorph, $\mathrm{CaCO}_{3}, \mathrm{~K}, \mathrm{Na}$ ), probably reflecting sharp fluctuations of river load characteristic of the Interglacial periods.
3) Sedimentation in the central Black Sea (Site 379) during most of the Quaternary was contributed from the Pontic drainage area enriched in montmorillonite and clinopyroxenes, derived from volcanogenic rocks of basic and ultrabasic composition. $\mathrm{Cr}, \mathrm{V}, \mathrm{Ni}, \mathrm{Co}, \mathrm{Sc}$, and, to some extent, $\mathrm{Ti}$ and $\mathrm{Fe}$, were relatively abundant. The lower half of the section was influenced by other sources from the northwest, the Crimean, and the Caucasian drainage areas.

In the Black Sea-Bosporus region, sedimentation was mainly influenced by the Danube, the Dnieper, and the Dniester, which drained sedimentary and acid volcanic rocks. In the site region, only the most finely dispersed products of acid rocks weathering (illite, quartz, feldspars) accumulated, since their supply areas were distant. In some instances, increased contents of $\mathrm{Cr}, \mathrm{V}$, $\mathrm{Ni}, \mathrm{Ti}, \mathrm{Fe}$, and other elements occur in Pliocene sediments, suggesting accumulation of basic rock weathered products.

4) The intensity of diagenesis of the sedimentary material was not the same at all sites. The weakest diagenesis occurs in Hole 379A, where diagenetic sulfides, small amounts of chemogenic diagenetic 
in $\mathrm{fr}, \mathrm{B},<0.001, \%$

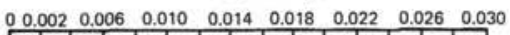

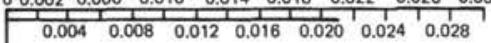

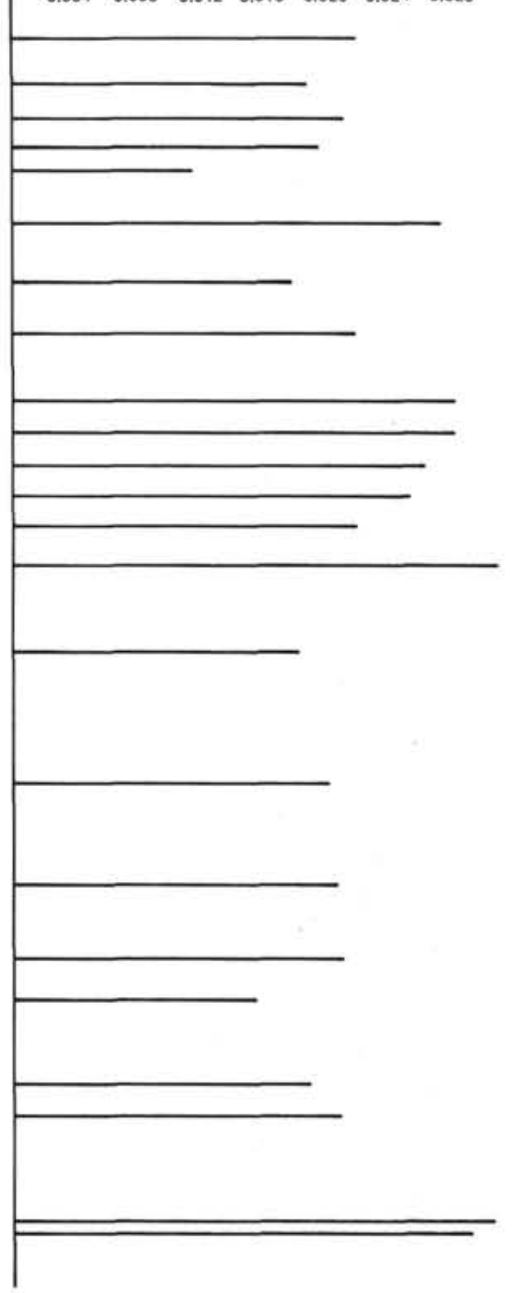

Beq, \%

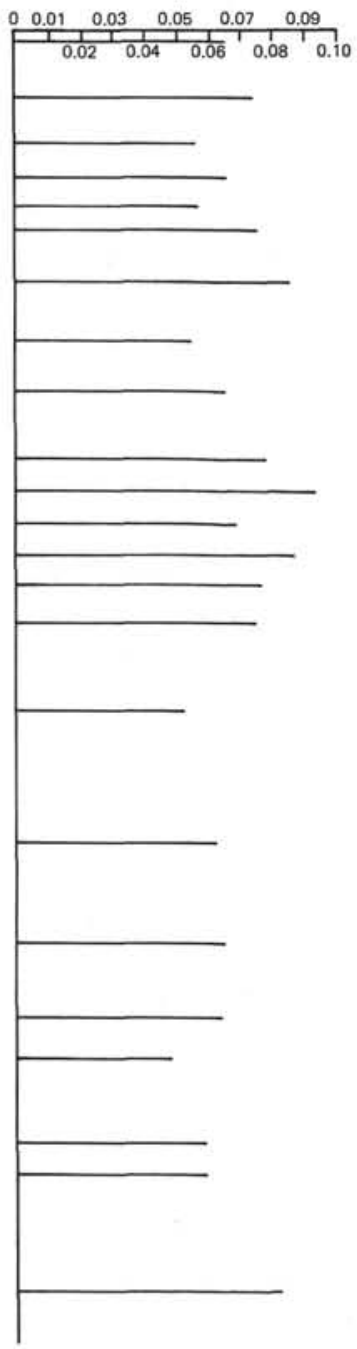

$\mathrm{K}_{2} \mathrm{O} \%$

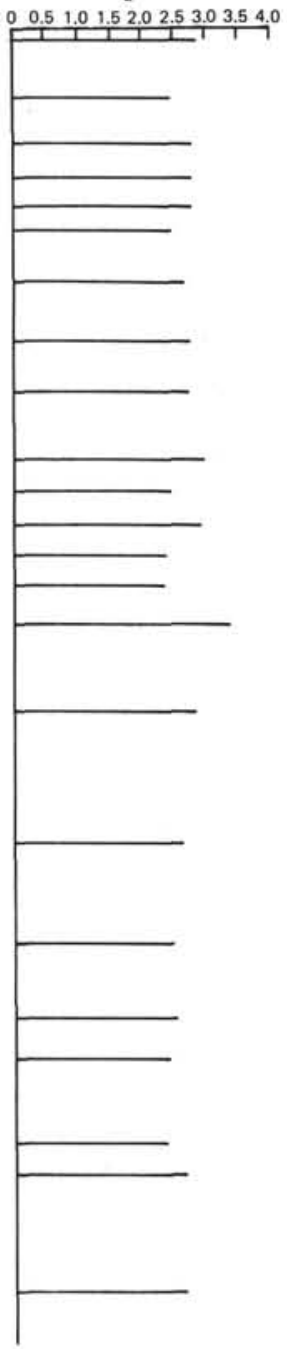

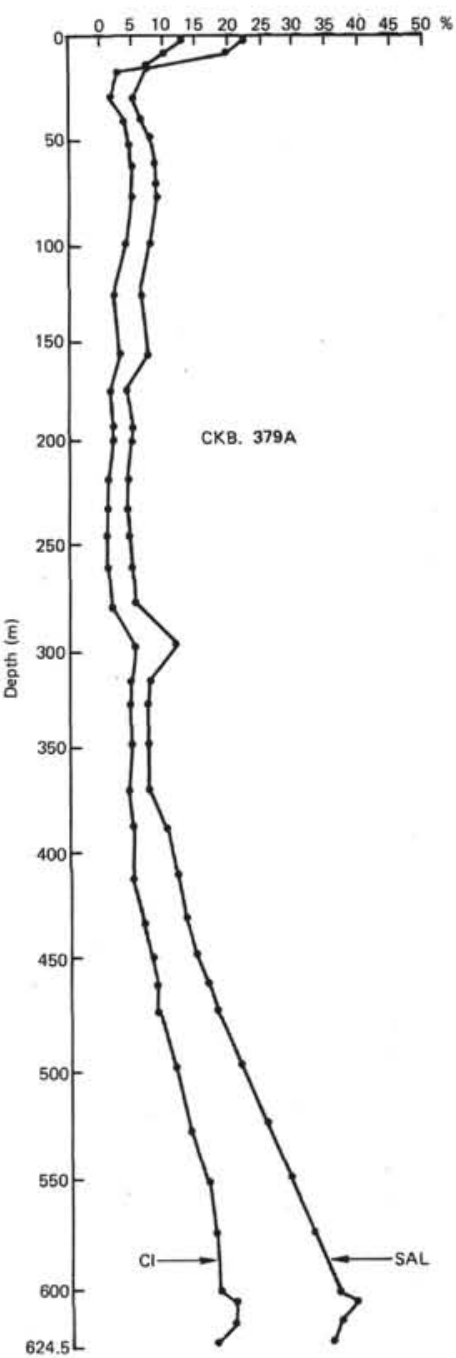

calcite, dolomites and (extremely seldom) aragonite occur.

The most intensive diagenesis took place in Holes $380 / 380 \mathrm{~A}$, and 381 , particularly in lower Neogene sediments. This is manifested in abornmally high interstitial water mineralization and sediment enrichment in organic matter. Besides large amounts of sulfides, diagenesis formed most of the calcite and dolomite. It is also responsible for the formation of aragonite, siderite, and $\mathrm{Mn}$-siderite as well as of clayey dolomite and sideritic interbeds. The latter are characterized by maximum $\mathrm{Fe}$ (up to $35.5 \%$ ) and $\mathrm{Mn}$ (up to $5.43 \%$ ) contents.

The formation of sulfides resulted in minor mobile elements redistribution $(\mathrm{Ni}, \mathrm{Co}, \mathrm{Mo}, \mathrm{Zn})$ with increased concentrations found in some interbeds enriched in organic matter.

The diagenetic formation of carbonates strongly affected the $\mathrm{Fe}, \mathrm{Mn}, \mathrm{Ca}^{2+}$, and $\mathrm{Mg}^{2+}$ distributions, whereas the minor element behavior was influenced insignificantly. Siderite interbeds are poorly enriched in $\mathrm{Mo}$ and $\mathrm{Se}$ (?).
5) Sedimentation in the Glacial periods and Holocene probably took place partly under anoxic conditions, as corroborated by interbeds with a high organic content. A necessary condition for stagnant Black Sea deep water formation is inflow of saline Mediterranean waters (see the curve of interstitial water salinity for Hole 379A; Hunt, this volume), and is also apparent in the boron distribution at all the sites. Anoxic periods can also be identified by mollusc composition in the coastal terrace deposits (Muratov, 1960; Fiodorov, 1963). The reduced sediments are more highly enriched in those elements which co-precipitate with sulfides (Mo, Se, and probably $\mathrm{Zn}, \mathrm{As}$ ), but impoverished in those elements which are easily dissolved in the highly reducing medium (Mn, partly $\mathrm{Fe}, \mathrm{Ni}, \mathrm{Co}$ ). All these characteristics were also observed in the Interglacial series.

6) Fluctuations of sea level also affected the geochemical differentiation of sedimentary material during late Cenozoic periods. During some Interglacial epochs, when sea level was high, those elements associated with the finely dispersed fraction of the river 
loads were transported into the sea in increased amounts and were delivered into the more distant parts of the sea.

In the Glacial epochs, when the sea level was lowered and the rate of sedimentation increased, the differentiation of the sedimentary material became significantly weaker, being affected mainly by suspended matter flows. Large amounts of coarsegrained material were transported into the deep sea. Thus, glacial periods were favorable for accumulation of those elements associated with terrigenous clastic material ( $\mathrm{Al}, \mathrm{Ti}, \mathrm{Zr}, \mathrm{Sn}, \mathrm{Cr}$, etc.).

(7) Average element contents in the Black Sea Neogene-Pleistocene sediments are similar to those of the Atlantic Ocean Recent terrigenous sediments. In the Black Sea deposits, only those elements usually associated with sulfides ( $\mathrm{Zn}, \mathrm{Mo}, \mathrm{Se}$, etc.) and sapropels are more abundant; in contrast, in the open oceanic sediments, biogenic components $\left(\mathrm{CaCO}_{3}\right.$, $\mathrm{SiO}_{2}$ amorph, and those elements which coprecipitate with $\mathrm{Fe}$ and $\mathrm{Mn}$ hydroxides) are more abundant. In some cases, the oceanic sediments appear to be richer in those elements associated with basic pyroclastics $(\mathrm{Fe}, \mathrm{Ti}, \mathrm{V}$, $\mathrm{Cr}, \mathrm{Ni}, \mathrm{Co}$ ).

\section{REFERENCES}

Andrusov, N. I., O verkhnepliotsenovykh otlozheniyakh mysa Chauda na Kerchenskom poluostrove. (On the Upper Pliocene deposits of Cape Chauda on the Kerch Peninsula): Trudy St. Petersb. Obshch. est., otd. geol. i min., prot. zased., v. 20.

Arkhangelsky, A. D. and Strakhov, N. M., 1938. Geologicheskoe stroenie i istoriya pazvitiya Chernogo morya. (Geological compsoition and the Black Sea development history): Akad. Nauk SSSR, M-L, p. 1-310.

Berner, R. A., 1965. Dolomitization of the Mid-Pacific atolls: Science, v. 147, p. 1297-1299.

Boyko, T. F., 1964. Lity, rubidy, tzezy. V kn. "Metally v osadochnykh tolshchakh". (Lithium, rubidium, cesium. From “Metals in sediments"): Nauka, M., p. 392-421.

Brewer, P. G. and Spencer, W., 1974. Distribution of some elements in the Black Sea and their flux between dissolved and particulate phases. In Degens, E. T. and Ross, D. A. (Eds.), The Black Sea-geology, chemistry, and biology: Am. Assoc. Petrol Geol. Mem. 20, p. 137-143.

Degens, E. T., 1971. Sedimentological History of the Black Sea over the last 25,000 years. In Campbell, A. S. (Ed.), Geology and history of Turkey, p. 407-429.

Friedman, D. M. and Sanders, D. I., 1970. Genezis i rasprostranenie dolomitov. $\mathrm{Kn}$. "Karbonatnye porody". Genezis, rasprostranenie, klassifikatziya. (Dolomite genesis and distribution. From "Carbonate rocks." Genesis, distribution, classification.): Izd. "Mir," M., v. 1, p. 249-319.

Glagoleva, M.A., 1959. Formy migratsii elementov v rechnykh vodakh. Kn. 'K poznaniju diagenesa osadkov'. (Element migration forms in river waters. From "On sediment diagenesis study”.) Izd. Akad. Nauk SSSR, M.

, 1961. K geokhimii osadkov Chernogo morya. V sb. "Sovremennye osadki morei i okeanov". (On geochemistry of the Black Sea sediments. From "Recent sediments of seas and oceans"). Izd. Akad. Nauk SSSR, M., p. 448-476.

Gursky, 1971. Geokhemicheskoe povedenie bora, joda $\mathrm{i}$ broma $\mathrm{v}$ sovremennykh morskikh otlozheniyakh.
(Geochemical boron, iodine and bromine behavior in recent sea deposits): Tezisy dokladov, c. 2, Mezhd. geokhim. kongressa, M.

Hirst, D. M., 1974. Geochemistry of sediments from eleven Black Sea cores. In Degens, E. T. and Ross, D. A. (Eds.), The Black Sea-geology, chemistry, and biology: Am. Assoc. Petrol. Geol. Mem. 20, p. 430-455.

Hunt, J. M., 1974. Hydrocarbon geochemistry of Black Sea. In Degens, E. T. and Ross, D. A. (Eds.), The Black Sea-geology, chemistry and biology: Am. Assoc. Petrol. Geol. Mem. 20, p. 499-504.

Kurinov, A. D., 1975. Instrumentalny neitronnoaktivatzionny analiz okeanicheskikh osadkov. (Instrumental neutron-activation analysis of oceanic sediments): Avtoreferat dissertatzii, Institut okeanologii Akad. Nauk SSSR, M.

Kurinov, A. D., Lisitzin, A. P., Lukashin, U. N., and Smakhtin, L. A., 1974. Neitronno-aktivatzionnye i spektralnye metody opredeleniya mikroelementov $v$ okeanicheskikh osadkakh. (Neutron-activation and spectral methods for determination of microelements in oceanic sediments): Okeanologiya, v. 24, p. 555.

Kuznetzov, Yu. V., 1971. Radiokhimicheskie osnovy neravnovesnykh metodov datirovaniya okeanicheskikh osadkov. (Radiochemical bases of nonequilibrium methods for oceanic sediment dating): Avtoreferat dissertatzii na soisk. uchenoy stepeni d-ra khimicheskikh Nauk, L, p. 1-32.

Lubchenko, I. Yu. and Belova, I. V., 1973. Migratziya elementov $\mathrm{v}$ rechnykh vodakh Chernomorskogo basseina. (Migration of elements in river waters of the Black Sea basin): Litologiya i poleznye iskopaemye, no. 2.

Lisitzin, A. P., 1964. Raspredelenie i khimichesky sostav vzvesi v vodakh Indiyskogo okeana. (Distribution and chemical composition of suspended matter in the waters of the Indian Ocean): Okeanologiya (rezultaty MGG), no. 10, Nauka, M, p. 1-136.

Meishtas, E. A., 1969. K metodike kolichestvennogo spektralnogo analiza nekotorykh mikroelementov $\mathrm{v}$ morskikh donnykh osadkakh (Ba, Be, Ge, Cr, Ni, Mo, Zr, V). (On the methods of quantitative spectral analysis of some microelements in marine bottom sediments): Okeanologiya, v. X, p. 374-382.

Müller, G. and Stoffers, P., 1974. Mineralogy and petrology of Black Sea basin sediments. In Degens, E. T. and Ross, D. A. (Eds.), The Black Sea-geology, chemistry and biology: Am. Assoc. Petrol. Geol. Mem. 20, p. 200-248.

Muratov, M.V., 1960. Chetvertichnaya istoriya Chernomorskogo basseina $\mathrm{v}$ sravnenii $\mathrm{s}$ istoriey Sredizemnogo morya. (A comparison between the Quaternary history of the Black Sea basin and the Mediterranean). Byulleten MOIP, otd. geol., 35, no. 5.

Pilipchuk, M. F., 1967. Molibden v vode Chernogo i Azovskogo morey. (Molybdenum in the Black Sea and Azov Sea waters): Geokhimiya, no. 8.

1971. Geokhimiya molibdena v Chernom more. (Molybdenum geochemistry in the Black Sea): Avtoreferat dissertatzii, MGU, M, p. 1-32.

Pilipchuk, M. F. and Volkov, I. M., 1966. Volfram v sovremennykh osadkakh Chernogo morya. (Wolfram in recent Black Sea sediments): DAN SSSR, no. 2, p. 167. 1974. Behavior of Molibdenum in process of sediment formation and diagenesis in the Black Sea. In Degens, E. T. and Ross, D. A. (Eds.), The Black Sea-geology, chemistry, and biology: Am. Assoc. Petrol. Geol. Mem. 20, p. 542-553.

Poluektov, N. S., 1959. Metody analiza po fotometrii plameni. (The methods of analysis by flame photometry): M, p. 140-141. 
Ponomariov, A. I., 1961. Metody khimicheskogo analiza silikatnykh i karbonatnykh porod. (The methods of chemical analysis of silicate and carbonate rocks): $\mathrm{M}$, p. 140-141, 241-246.

Shimkus, K. M. and Trimonis, E. S., 1974. Modern sedimentation in Black Sea. In Degens, E. T. and Ross, D. A. (Eds.), The Black Sea-geology, chemistry and biology: Am. Assoc. Petrol. Geol. Mem. 20, p. 249-278.

Shimkus, K. M., Mukhina, V. V., and Trimonis, E. S., 1973. $\mathrm{O}$ roli diatomey $\mathrm{v}$ pozdnechetvertichnom osadkoobrazovanii Chernogo morya. (On the role of diatoms in late Quaternary sedimentation in the Black Sea): Okeanologiya, v. XIII, p. 1066-1071.

Shimkus, K. M., Emelyanov, E. M., Trimonis, E. S., 1975a. Donnye otlozheniya i cherty pozdnechetvertichnoy istorii Chernogo morya V monografii "Zemnaya kora i istoriya razvitiya Chernomorskoy vpadiny". (Sea-floor sediments and Late Quaternary history of the Black Sea. In The Earth's crust and the history of development of the Black Sea basin"): Izd. Nauka, M, p. 138-163. , 1975b. Stratigrafiya osadkov Chernogo morya i skorosti sedimentatzii. (Stratigraphy of deep-water Black Sea sediments and sedimentation rates): Informatzionny bulleten SEV, no. 3, p. 158-168.

Shishkina, O. V., 1959. Metamorfizatziya khimicheskogo sostava ilovykh vod Chernogo morya. Sb. "K poznaniyu diageneza osadkov',. (Metamorphization of chemical composition in the Black Sea mud waters. From "On the study of sediment diagenesis"): Izd. AN SSSR, M, p. 2050 .

1972. Geokhimiya morskikh i okeanicheskikh ilovykh vod. (Geochemistry of sea and ocean mud waters): Izd. Nauka, M, p. 1-228.

Sokolova, E. G. and Pilipchuk, M. F., 1970. Selen v. sovremennykh osadkakh Chernogo morya. (Selenium in recent Black Sea sediments): DAN SSSR, 1970, v. 193, p. 692.

Starik, I.E., Nikolaev, D.S., Kuznetzov, Yu. V., and Legin, V. K., 1961a. Radioktivnost osadkov Chernogo morya. (Radioactivity of the Black Sea sediments): DAN SSSR, v. 139 , p. $1456-1459$. 1961b. Sootnoshenie radioaktivnosti osadkov Azovskogo i Chernogo morey. (A radioactivity ratio in the Azov and Black Sea sediments): DAN SSSR, v. 139, p. 456-459.

Strakhov, N. M., 1971. Geokhimicheskaya evolyutziya Chernogo morya v Golotzene. (Geochemical evolution of the Black Sea during Holocene): Litologiya i poleznye iskopaemye, no. 3, p. 3-17.

Strakhov, N. M., Belova, I. V., Glagoleva, M. A., and Lubchenko, I. Yu., 1971. Raspredelenie i formy nakhozhdeniya elementov $v$ poverkhnostnom sloe sovremennykh chernomorskikh otlozheniy. (Distribution and mode of element occurrences in the surface layer of recent Black Sea sediments): Litologiya $\mathrm{i}$ poleznye iskopaemye, no. 2, p. 3-31.

Sukhorukov, F. V. and Emelyanov, E. M., 1969. Bor v donnykh osadkakh severovostochnoy chasti basseina Atlanticheskogo okeana. (Boron in bottom sediments of the north-eastern Atlantic Ocean basin): DAN SSSR, v. 187 , p. $1153-1156$.

Vital, D. A., 1959.. Karbonatnye konkretzii v mezozoiskikh otlozheniyakh Russkoy platformy. (Carbonate concretions in Mesozoic sediments of Russian Platform). From K poznaniyu diageneza osadkov": Izd. AN SSSR, M, p. 196-237.

Volkov, I. I., 1975. Khimicheskie elementy v rechnom stoke i formy ikh postupleniya v more (na primere rek Chernomorskogo basseina). Sb. "Problemy litologii i geokhimii osadochnykh porod i rud". (Chemical elements in a river drain and forms of their supply into the sea. In "Problems of lithology and geochemistry of sedimentary rocks and ores): Izd. Nauka, M, p. 85-113.

Volkov, I. I. and Fomina, L. S., 1974. Influence of organic material and processes sulfide formation on distribution of some trace elements in deep-water sediments of the Black Sea. In Degens, E. T. and Ross, D. A. (Eds.), The Black Sea-geology, chemistry and biology: Am. Assoc. Petrol. Geol. Mem. 20, p. 445-476.

Walker, C. T. and Price, N. P., 1963. Departure curves for computing palaeosalinities from boron in illites and shales: Assoc. Petrol Geol. Bull., v. 47, p. 833. 